\title{
Reservoirs in the United States
}

By $\mathrm{R} O \mathrm{O}$ MARTIN and RONALD L HANSON

GEOLOGICAL SURVEY WATER-SUPPLY PAPER 1838

Revising and superseding Geological Survey Water-Supply Paper 1360-A, Reservoirs in the United States, by Nathan O. Thomas and G. Earl Harbeck, Jr., 1956

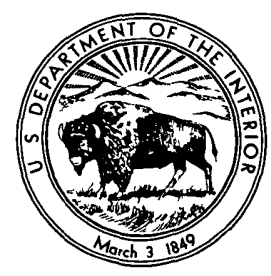




\title{
UNITED STATES DEPARTMENT OF THE INTERIOR
}

STEWART L UDALL, Secretary

\author{
GEOLOGICAL SURVEY \\ William T. Pecora, Director
}

Library of Congress catalog-card No GS $66-276$ 


\section{PREFACE}

This report on reservours in the United States was prepared in the Water Resources Division, L B Leopold, chief hydrologist, under the general supervision of $\mathrm{E} \mathrm{L} \mathrm{Hendricks,} \mathrm{and} \mathrm{subsequently} \mathrm{M}$ R Williams, chiefs, Surface Water Branch. It revises and supersedes Water-Supply Paper 1360-A, by N O Thomas and G E Harbeck, $\mathrm{Jr}$, which in turn superseded Geological Survey Circular 23, by G E Harbeck, Jr This text is largely the work of the original author as presented in Geological Survey Circular 23. All three reports were prepared with the advice of $W$ B. Langbein

The basic data were obtamed directly from the owner or operator of the reservorr whenever practicable Complete data were not avdllable for all reservorrs

Acknowledgement is made to the following agencies and organizations for their cooperation in making the reservoir information avallable the US Army Corps of Engineers, the Bureau of Reclamation, the Tennessee Valley Authority, the Soll Conservation Service, and several other Federal agencies, agencies of States, counties, and municipalities; water users associations, conservation and irrigation districts, flood control and conservation districts; many power, water, canal, and reservoir companies, as well as many interested individuals.

In bringing this complation up to date every effort was made to obtain the results of the most recent surveys to determine the reservoir capacity Possibly some of the data published herem may inadvertently be in error, and any corrections or additions that can be furnished by the user of these records will be appreciated 



\section{CON TEN TS}

\begin{tabular}{|c|c|}
\hline \multicolumn{2}{|c|}{ Page } \\
\hline \multirow{2}{*}{ 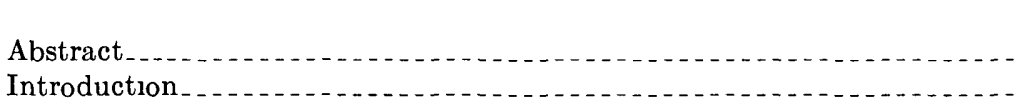 } & \\
\hline & \\
\hline & \\
\hline Ir and stream & \\
\hline servorr & \\
\hline (2, & \\
\hline & \\
\hline 思 & \\
\hline 㐌 & \\
\hline 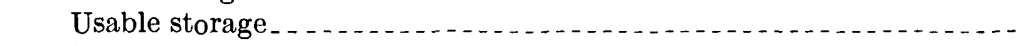 & \\
\hline 10 & 1 \\
\hline מ & 1 \\
\hline 1 & 12 \\
\hline tor & 1 \\
\hline blished & 1 \\
\hline States . . . . . . . & 11 \\
\hline Jacent States. . . . . . & 11 \\
\hline - n & 11 \\
\hline & \\
\hline
\end{tabular}

\section{PIATE}

Plate 1 Map of the conterminous United States showing drainage areas

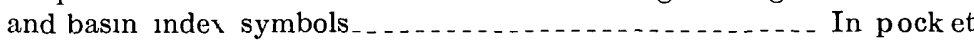

\section{GRAPHS}

Figure 1 Usable capacity of reservoirs in the United States

2 Usable reservoir capacity for power, installed generating capacity, and production of hydroelectric power........... 6

3 Number and average capacity of reservoirs constructed by decades. 


\section{TABLES}

TABLE 1 Reservoirs in the United States completed or under construcPage tion, as of Jan 1, 1963, having a usable capacity of 5,000 acre-feet or more. . . . . . . . . . . . . . . . . . . . . . . .

2 Summary of number, usable storage, and surface area of reser-

3 Reservors on streams which form a boundary between adjacent

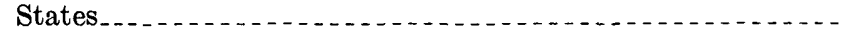




\title{
RESERVOIRS IN THE UNITED STATES
}

\author{
By R. O. R Martin and Ronald L Hanson
}

\section{ABSTRACT}

This report summarizes the storage capacities and related data of reservoirs and controlled natural lakes for the conterminous United States, Alaska, Hawan, and the Commonwealth of Puerto Rico Data are given for all storage facilities having a usable capacity of 5,000 acre-feet or more and completed or under construction as of Jan 1, 1963

A descriptive list of reservoirs in the United States completed as of Jan 1, 1947, was first published (Geol Survey Circular 23) in March 1948 In 1956 Water-Supply Paper 1360-A, "Reservoirs in the United States," by N O Thomas and G E Harbeck, Jr, updated this listıng and included lists of reservours completed or under construction as of Jan 1, 1954 Some of the data shown for reservoirs constructed before 1954 may have been corrected herein on the basis of the latest avallable reservoir survey

This report lists 1562 reservoirs and lakes, their usable storage totals $359,360,000$ acre-feet, and the corresponding surface area is $14,831,000$ acres

\section{INTRODUCTION}

Man has engaged in the control of flowing water since history began Among his early recorded efforts were reservoirs for municipal water supplies constructed near ancient Jerusalem to store water brought there in masonry conduits (Draffin, 1939, p 122-127). Irrigation was practiced in Egypt as early as 2000 B C by the Pharoahs. There the "basin system" was used untll the 19th century; to store water the land was divided into basins of approximately 40,000 acres and separated by earthen dikes (Todd, 1929, p 536) The system was effective if flood waters of the Nile River flowed through canals and inundated these basins; however, in years of no floods the crops suffered In India, the British found at the time of their occupancy of the Presidency of Madras about 50,000 reservolrs which were used for irrigation; many of these reservoirs were believed to be of ancient construction (Turneaure and Russell, 1909) Reservoirs were bult to improve the water supply of Athens during the period A D 115130. Much has been written concerning the elaborate collection and distribution sys'em bult to supply Rome, and parts of the system stıll 
remain as monuments to the engineering skill of the Romans in solving the problems of large-scale municipal water supplies

The need for adequate and controllable water supplies for the purposes of irrigation, municipal supply, power, and flood control has long been recognized Unfortunately, man cannot effectively control the amount or time of precipitation, therefore, he has utilized water storage to overcome the variation in water supply Water storage has contributed materially to the economic development of the United States In the semiarid sections of the West, the successful agricultural economy depends largely upon storage of a part of the annual water supply for use during the growing season The growth of our vast industrial centers has been made possible only by means of adequate water supplies throughout the entıre year for municipal and industrial use The industrial expansion in many regions has paralleled the increase in avallable hydroelectric power supplies The construction of flood-control reservorrs has contributed materially to the protection of downstream urban, municipal, and industrial developments by retention of floodflows

The interest of the US Geological Survey in the reservolrs of the United States may be traced back to 1888, when the Sundry Civil Appropriation Act of that year provided (Follansbee, 1939, p 33)

For the purpose of investigating the extent to which the arid region of the United States can be redeemed by irrigation and the segregation of the irmgable lands in such arid region, and for the selection of sites for reservoirs and other hydraulic works necessary for the storage and utilization of water for irrigation and the prevention of floods and overflows, *** the work to be performed by the Geological Survey under the direction of the Secretary of the Interior, the sum of one hundred thousand dollars ***

The Irrigation Survey (1888-90), a branch of the Geological Survey, conducted the first systematic investigation of water resources in the United States During its 2-year life much was done to evaluate the water resources of the arid West The Irrigation Survey clearly demonstrated the need for comprehensive investigations of reservoir sites and of potential water supplies

Other legislation affecting the construction of reservoirs in the West mcluded the Carey Act of 1894, which authorized grants of public lands to the Western States provided that such granted land would be irrigated, reclaiming the arid lands however, was slow partly because the large size and great cost of many of the necessary projects far exceeded the resources of any single State or private interest

The great development of the arid West near the turn of the century was made possible laigely by the construction of storage reservoirs; where precipitation was insufficient for agriculture, reservoirs were needed to hold spring floods caused by melting snow and to retain 
rapid runoff from short, but intense, summer storms The first reservoir possessing a capacity of 5,000 acre-feet or more and having a provision for irrigation was probably French Lake, built in 1859, in northern California Few large reservoirs were bult before $1900 \mathrm{~m}$ the West, but thereafter construction proceeded much more rapidly and by 1910 the capacity of reservoirs for irrigation exceeded the capacity of reservoirs for any other use (fig 1) Total capacity for hydroelectric power in the thirties in single and multipurpose reservoirs forged ahead of capacity for irrigation purposes, and total capacity for flood control in the early fifties overtook irrigation capacity even though the capacity for irrigation has maintained a steady growth

The Reclamation Act, or Newlands Act, of 1902 provided Federal and for the construction of reservoirs This legislation stimulated the construction of reclamation works by providing a method of amortizing heavy initial costs over many years Because of this legislation, the development of the arid West advanced in the early years of this century in a way that it could not have done otherwise The settlement of the West proceeded rapidly as reservoirs were bult to assure an adequate supply of water for agriculture

Most of the irrigated farmlands are in the 17 Western States and, according to the Bureau of the Census, the following acreages in these States were irrigated by surface water

\begin{tabular}{|c|c|}
\hline Year & Acreage \\
\hline 1919 & $16,317,451$ \\
\hline 1929 & $16,073,246$ \\
\hline 1939 & $20,354,239$ \\
\hline 1949 & $19,272,564$ \\
\hline 1959 & ${ }^{1} 22,000,000$ \\
\hline
\end{tabular}

When these figures are compared with the usable reservolr capacity for irrigation at 10-year intervals, as shown in figure 1, it can be seen that farmland acreage irrigated by water from streams and lakes has increased at a much slower rate than the reservolr capacity for irrigation These figures indicate that the acreage irrigated from surface water in the Western States has, to some extent, become stabiluzed at about 20 million acres. Two other conclusions can probably be drawn from the comparison irrigation systems in recent years have required more storage per urrigated acre than earler systems, and much of the large increase, since 1920, in storage of water for irrigation has been utılized for the purpose of reducing crop fallure on land that is already being irrigated 


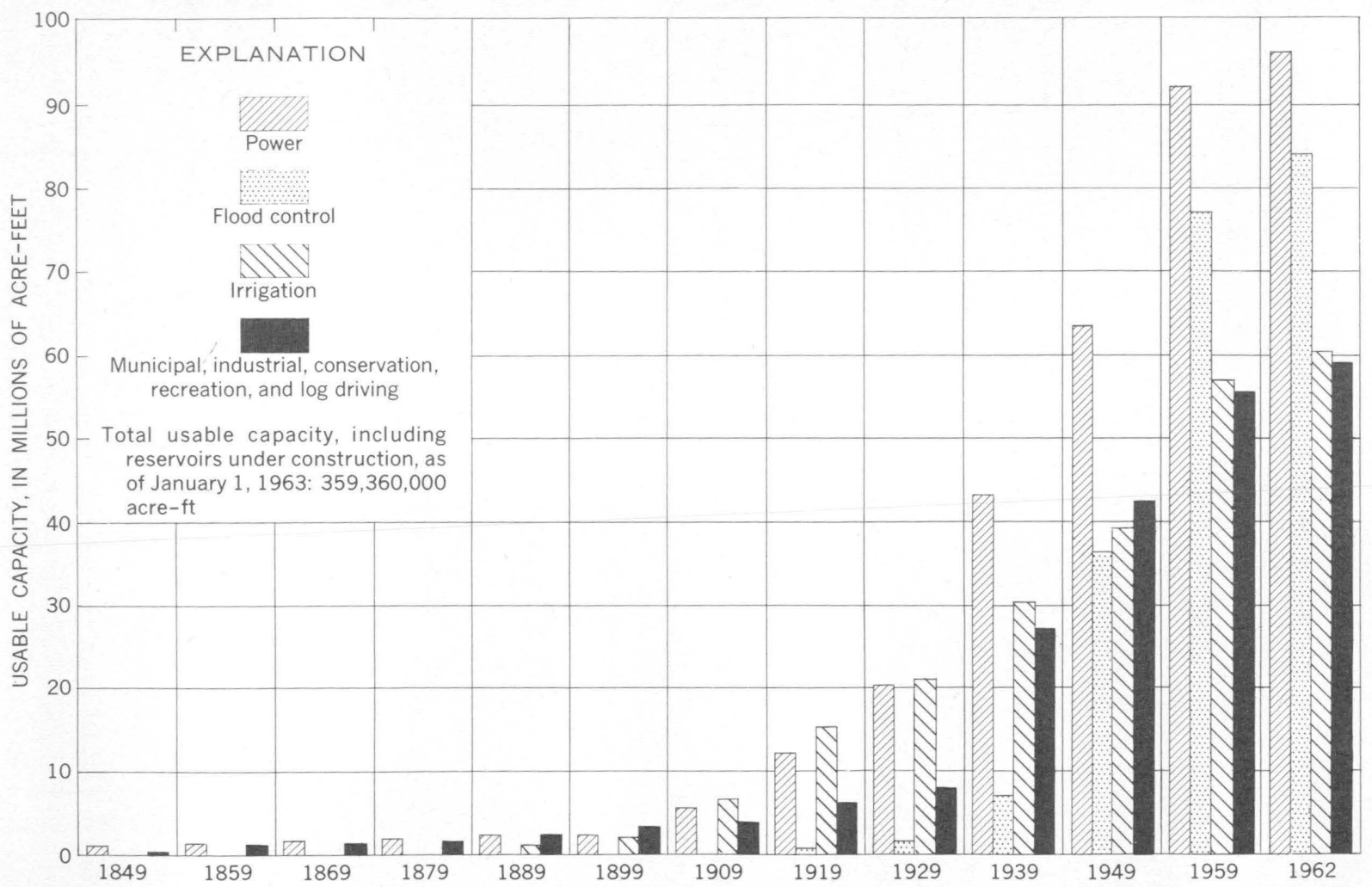

Figure 1.-Usable capacity of reservoirs in the United States, 1849-1962. 
The acreage of land fully or supplementally irrigated by systems constructed by the Bureau of Reclamation for 10-year intervals is as follows

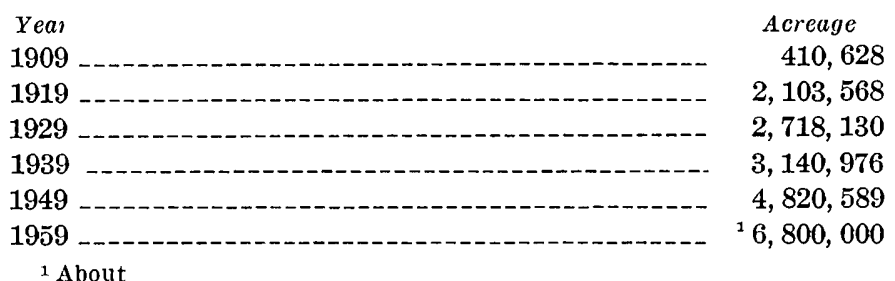

These same areas are mcluded in the census figures previously given for the 17 Western States During the 1919-59 period, acreage 1rr1gated by the Bureau of Reclamation works increased about 3 times and followed very closely the increase in storage for irrigation as shown in figure 1

Reservolrs were constructed to supply water power long before the advent of hydroelectric power, but after the transmission of electricity over long distances became possible, the construction of hydroelectric reservolrs began an extraordinary growth The first hydroelectric station began operation in 1882 in Appleton, Wis (Daugherty, Horton, and Davenport, 1928, p. 29) Rapid growth in reservolr construction for power occurred in New England during 1890-1910 Hydroelectric reservolr construction has continued at a rapid pace, with great increases in the Pacific States since 1910, in the South Atlantic States during 1920-30, and in the Mountain States during 1930-40 Completion of Lake Mead (Hoover Dam) and the reservoirs in the Tennessee Valley contributed greatly to the consistently sharp increases during the period 1930-50 shown by figure 1 The greatest increase in reservoir construction tor power supply was ind1cated during the 1950-59 decade By January 1963, about 32 percent of the total reservoir capacity in the United States was allocated for hydroelectric power production

Installed hydroelectric generating capacity and the production of hydroelectric power are compared with usable reservolr capacity for power in figure 2 All three factors have consistently shown an upward trend The greatest increase in installed generating capacity (15 2 milhon kilowatts) took place during the 1950-59 decade along with the great increase in reservorr storage for power during this period According to the Federal Power Commission (1964) 38 million kllowatts, about 25 percent of the country's potential hydroelectric capacity, was made avallable by January 1963 This is more than twice the generating capacity of reservoirs in 1950 
The production of power, which is largely dependent on demand, has increased at about the same rate as the increases in installed generating capacity and usable reservoir capacity for power. Annual production of hydroelectric power reached about 190 billion kilowatthours in 1962, which is more than double the power production of 1950 .

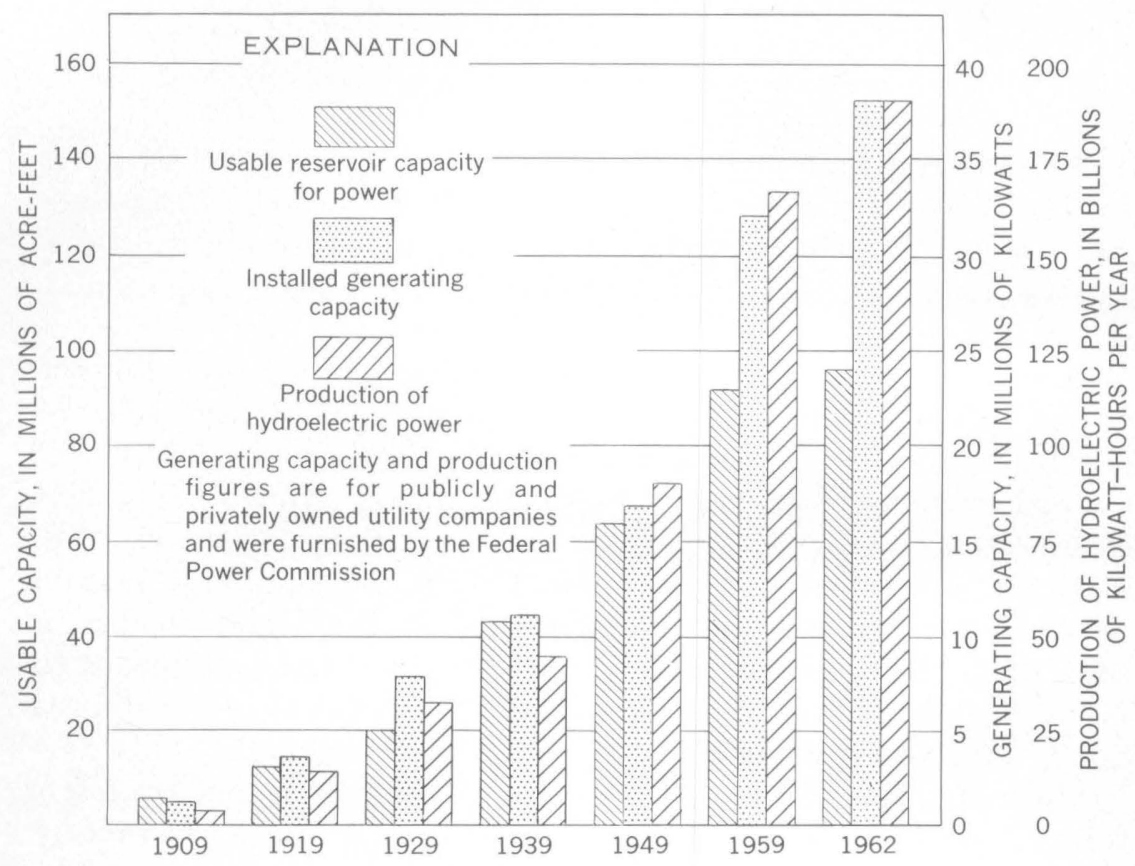

Figure 2.-Usable reservoir capacity for power, installed generating capacity, and production of hydroelectric power, 1909-62.

Construetion of reservoirs for flood control received its first notable impetus in the early twenties with the completion of a reservoir network in the Miami River basin of Ohio, built as a consequence of the disastrous flood of 1913. Since 1930, the construction of floodcontrol reservoirs and the allocation of capacity for flood control in multipurpose reservoirs has increased at a greater rate than that for any other use so that now the total reservoir capacity for this purpose is surpassed only by the capacity allocated for power. Including reservoirs under construction on Jan. 1, 1963, an increase of 48 million acre-feet in flood-control capacity has occurred since $1950-$ the greatest increase for any use to date. This increase is due primarily to the construction of reservoirs in the Missouri River basin and in the States of Texas and Arkansas. 
The present complation of reservoirs in the United States is an inventory of facllities for the use and control of water, one of our most important natural resources The purposes of reservoir construction reflect the changes $i n$ the national economy since water storage began In colonial days and in the early years of the Republic, many low dams and small storage reservoirs were bult for small industries and for log driving The earliest recorded effort to store a substantial amount of water was that in New Hampshire, where records show that a control structure bult at the outlet of Massabesic Lake in 1738 increased the storage capacity of the lake by 19,000 acre-feet This early structure was bult to operate a gristmill and a sawmill Most of the large reservoirs constructed in the early half of the 19th century were built to insure adequate water supplies for the canal systems then in use Although some of these old reservorrs are still used to supply canals, many are now used only for recreation and their original purpose is all but forgotten.

During the latter half of the 19th century, the water needs of rapidly growing cities in the East were outstripping their local supplies As a result, this period has an appreciable increase in reservoir construction for municipal supplies

One of the earliest large reservoirs for navigation was Cayuga Lake in New York, where a dam was bultt in about 1825 to raise the level of the lake to provide supplemental water for the Erie Canal The outlet structure for Lake Drummond in Virginia was also constructed about the same time to provide water for slack-season navigation The growth of reservoir capacity for navigation was slow, and in the latter half of the 19th century, many of these reservours were abandoned During past years, however, outstanding growth has taken place with the completion of multipurpose projects having substantial allocations for navigation Most of this growth was due to multipurpose projects in the Missourı and Tennessee River basins Many reservolrs have also been created by dams constructed specifically to maintain navigable depths on streams such as the Ohıo and upper Mississippi River Most of these reservoirs are not included in the present complation because they are not storage reservoirs in the usual sense of storing water for later release

The following list of reservolrs, in order of size, are those $m$ the United States that have usable capacities of 2 million acre-feet or more All these reservorrs, except Panted Rock, are multipurpose, and all are in public owneiship On Jan 1,1947, there were only nine multipurpose reservoirs having usable capacities of 2 million acrefeet or more The increase to 31 on Jan 1, 1963, exemplifies the trend toward large multipurpose projects in recent years 
Reservorrs in the United States having usable capacities of 2 million acre-feet or more

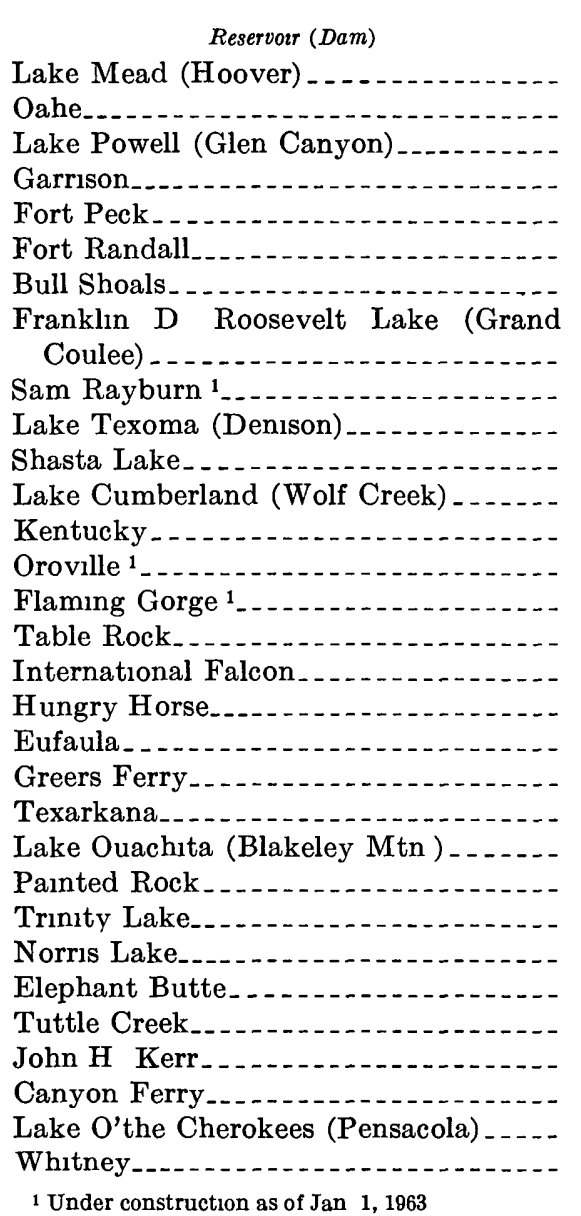

\begin{tabular}{|c|c|}
\hline State & $\begin{array}{c}\text { Usable capacity } \\
\text { (acre-feet) }\end{array}$ \\
\hline Arızona-Nevada........ & $27,207,000$ \\
\hline South Dakota_....... & $23,628,000$ \\
\hline Arizona & $20,876,000$ \\
\hline North Dakota $\ldots$ & $19,900,000$ \\
\hline Montana & $13,915,000$ \\
\hline South Dakota & $6,093,000$ \\
\hline Arkansas . . . . . & $5,399,600$ \\
\hline Washington_-. & $5,231,700$ \\
\hline Texas. $\ldots$ & $4,478,800$ \\
\hline Oklahoma-Texas _. . . . & $4,424,000$ \\
\hline Calıfornia_. _. & $4,377,000$ \\
\hline Kentucky _......... & $4,236,000$ \\
\hline Kentucky $\ldots$ & $4,010,800$ \\
\hline Calıfornia _ . & $3,498,600$ \\
\hline Utah & $3,515,700$ \\
\hline Missourı _. & $3,462,000$ \\
\hline Texas_. & $3,277,880$ \\
\hline Montana $\ldots \ldots$ & $2,982,000$ \\
\hline Oklahoma & $2,951,000$ \\
\hline Arkansas_. & $2,843,860$ \\
\hline Texas_...... & $2,654,300$ \\
\hline Arkansas_. & $2,602,000$ \\
\hline Arizona_. & $2,492,000$ \\
\hline Calıfornia _. . . . & $2,437,700$ \\
\hline Tennessee_......... & $2,281,000$ \\
\hline New Mexico & $2,195,000$ \\
\hline Kansas_.............. & $2,134,000$ \\
\hline Virgınia $\ldots \ldots$ & $2,110,500$ \\
\hline Montana $\ldots \ldots$ & $2,043,000$ \\
\hline 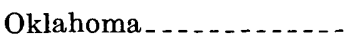 & $2,017,000$ \\
\hline Texas & $2,012,400$ \\
\hline
\end{tabular}

Of the privately-owned reservoirs, the Jargest is Lake Murray in South Carolina, completed in 1930 for power and having a usable capacity of 1,614,000 acre-feet Among the reservolrs used primarily for municipal purposes, Quabbin Reservoir in Massachusetts, completed in 1939, holds first rank and has a usable capacity of 1,236,000 acre-feet

The graph in figure 3 shows the number and average capacity of reservolrs constructed by decades The large increase in the number of reservoirs constructed during 1900-09 was almost entirely due to the Reclamation (Newlands) Act of 1909 Countless reservoirs of less than 5,000 acre-feet capacity, which are not listed here were likewise built for irrigation during that same period The decline in the number of reservolrs constructed during the 1940-49 period may 


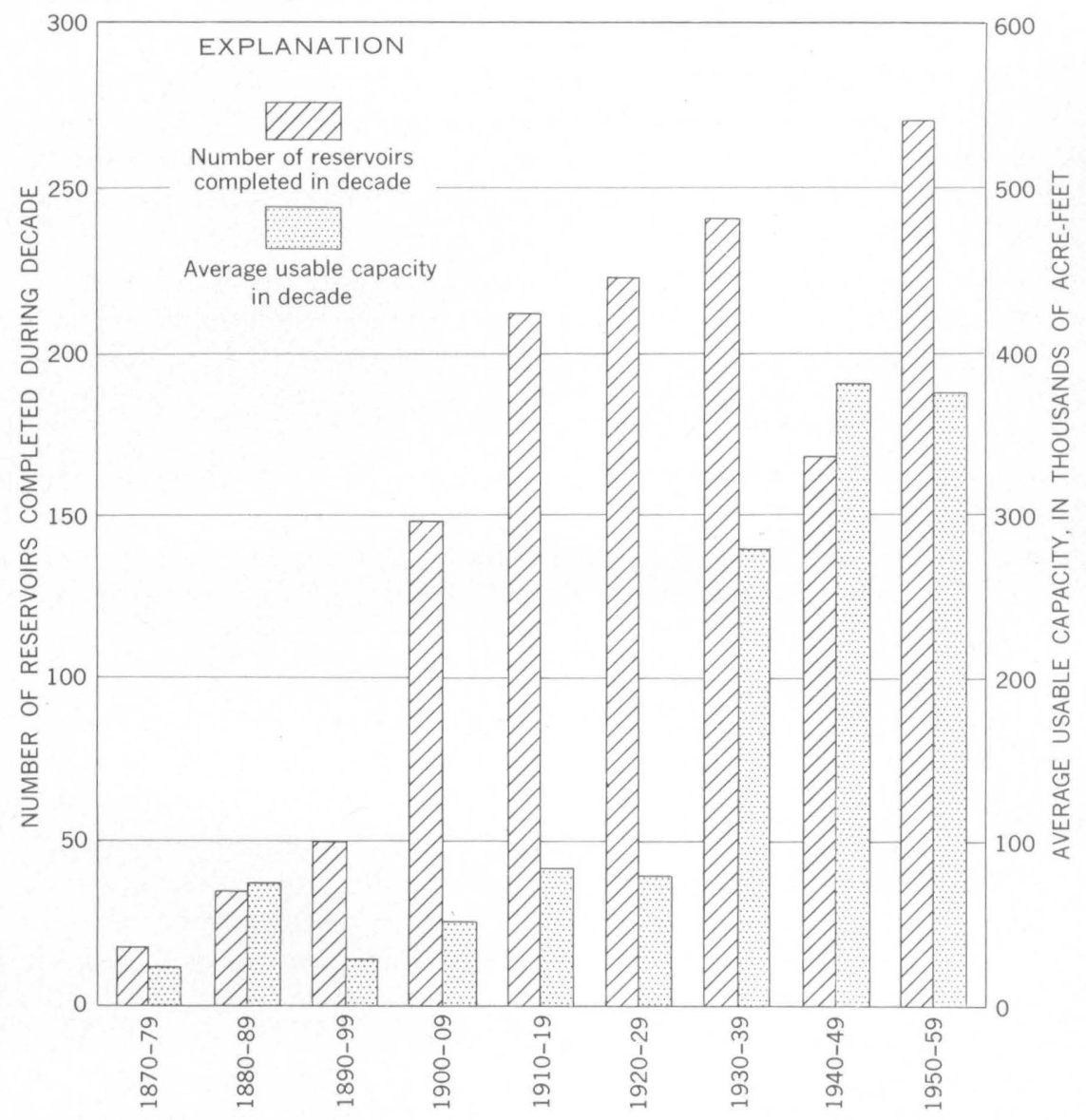

Figure 3-Number and average capacity of reservoirs constructed by decades, 1870-1959.

probably be attributed to World War II. Of the 430 reservoirs constructed since 1950, California leads with 66 new reservoirs, followed by Texas with 54 .

Technical advances have made feasible the construction of large projects that had long been desirable but hitherto had been considered impracticable. Thus, as the number of reservoir sites suitable for economic development were utilized and the demand for storage capacity for various uses increased, it was necessary to resort to reservoirs much larger than those previously constructed.

Federally-sponsored programs have resulted in a significant increase in the number of reservoirs having less than 5,000 acre-feet of storage capacity. Since 1948, watershed protection and flood pre- 
vention programs of the Department of Agriculture have resulted in the construction of 3,100 reservours having an average capacity of about 1,000 acre-feet each The total storage provided by these relatively small flood-control projects is not appreciable at the present time (1964) Reservoirs having less than 5,000 acre-feet of usable capacity have not been included in this compllation, however, if construction of small reservoirs for conservation and the prevention of floods, erosion, and sedıment damage continues, they could become much more important in water control and supply

\section{EXPLANATION OF COMPILED DATA}

Table 1 shows data for reservolrs and controlled natural lakes having a usable capacity of 5,000 acre-feet or more that were completed or under construction on Jan 1, 1963 Reservorrs are listed alphabetıcally by States Included are reservors in the Commonwealth of Puerto Rico

A descriptive list of reservoirs in the United States was completed Jan 1, 1947 (Harbeck, 1948) Water-Supply Paper 1360-A (Thomas and Harbeck, 1956) updated this list and gave the reservorrs completed or under construction as of Jan 1, 1954 Since preparation of Water-Supply Paper 1360-A, new surveys have been made at many reservolrs to obtain current data on storage and on rate of sedımentation The information given in table 1 is based on the latest avallable surveys, except in a few localities where the owner or operator indicated that recent surveys were not of sufficient accuracy to warrant a revision of previously published data At some reservoirs the operating condltions have been changed since 1954 or major construction work has affected the capacity, data shown in the table reflect these changes

It should be noted that the advance information shown for reservoirs under construction on Jan 1, 1963, is based on the premise that the reservoir will be completed in accordance with the owner's plans

As it is common practice in some regions of the United States to express reservoir capacities in units other than acre-feet, the following conversion factors may be useful

1 acre-foot $=03259$ million gallons;

1 acre-foot $=0.04356$ million cubic feet, 1 acre-foot $=05042$ cubic feet per second per day

\section{NAME OF RESERVOIR AND STREAM}

Reservoirs are listed alphabetically within each of the fifty States and Puerto Rico If the reser voir is on a boundary stream, common to adjacent States, the reservoir is listed under both of the States This duplication should be taken into account in any summation studies 
(table 3) If the stream on which the reservoir was bult flows across a State line and merely backwater from the dam extends across that line, the reservoir is listed only under the name of the State in which the dam or outlet structure was constructed

The reservolr name used is the latest legal name The name of the dam, if widely known, is given parenthetically if it differs from the name of the reservoir The water supply is generally derived from the stream on which the reservoir is situated, however, some reservoirs are on minor tributaries and are filled by diversions from the main stream For such reservoirs, the name of the stream furnishing the main supply is given, and the reservoir is shown by a footnote to be off the main stream

\section{LOCATION OF RESERVOIR}

The location shown in table 1 is that of the dam or control works for the reservoir and includes the drainage basin index, the station number, and the geographic coordinates of latitude and longitude

The basin index, made up of figures and letters, refers to the drainage area divisions shown on plate 1 The figures identify the 14 major dranage basms (or Parts) of the country, as classified and used by the Geological Survey The original Part 12 diannage area used in plate 1 of Water-Supply Paper 1360-A is divided in this report into Paits 12, 13, and 14 to conform to present classification divisions Other wise, the basin index symbols used in this compilation are the same as in pievious reports

The major dramage basins are divided as follows (Jones and Helland, 1948, p 3)

Each major area is divided into intermediate areas conforming to its dominant drainage systems and designated $12 \mathrm{~A}$. 12B, etc, the number in the designation being that of the major area and the lettel referrmg to a specific intermediate area within it The intermediate areas are further divided into minor areas, each designated by the number of the major area the letter of the intermediate area, and a final letter distınguishing it from adjacent minor areas, as 12FA, 12FB Each dramage basin division is lettered in order alphabetscally from the upper reaches of the basin to the lower

The station number is based upon the same system of downstream order identification used in the Geological Sur vey`s annual streamflow reports The complete station number tor each reser voir includes the major basin number (Pait number) followed by six digits indicating the downstieam order of the reservorr within the major basin Therefore, a reservoir with station number 13-3640 00 would be upstream from a reservoli having station number 13-3650 00 and both would be in the Snake River basin designated as Part 13

The geographic coordinates of the dam are given to the nearest minute of latitude and longitude 


\section{DRAINAGE AREA}

The drainage area at the dam is given where avalable and applicable In off-stream reservorrs the diamage area is omitted because in many of the reservoiss diversion does not bear any relation to the main-stream flow

\section{SURFACE AREA}

The surface area is the water area of the reservoir, in acres, at the elevation of the maximum controllable water level This level is generally at the spillway crest, without flashboards

\section{DEAD STORAGE}

Dead storage is the volume, in acre-feet, below the lowest controllable pool level The dead-storage figures shown in table 1 are based on normal operating conditions Normal operating conditions may not exist where sluiceways, below the lower limit of drawdown for power production, are opened The lower limit of drawdown for many multipurpose reservoirs is governed by the requirements of navigation, recreation, or conselvation, which may have been established by judicral decree or by agreements between the owners and other parties concerned The data in table 1 for those reservoirs subject to additional drawdown are generally based on normal operating conditions and are footnoted to indicate this fact In some reservoirs the dead-storage figure is show $n$ as zero when, in reality, it is unknown but considered negligible Dead-storage figures are generally not shown for natural lakes having permanent control structures at their outlets because this storage is usually unknown or of no practical interest

\section{TOTAL STORAGE}

Total storage is the volume, in acre-feet, below the maximum controllable pool level and includes dead storage For a dam having an ungated overflow spillway, it is the total volume below the spillway crest. When there is flow over the spillway, the total volume of stored water exceeds the total reservoir capacity, but such uncontrollable excess storage is excluded from this compilation Many dams have been designed to allow temporary detention of a considerable volume of flood water above the controllable pool level by the use of stoplogs or flashboards on the spillway crest Total storage figures based on the use of stoplogs or flashboards are footnoted as such in table 1 


\section{USABLE STORAGE}

The usable storage is the volume, in acre-feet, normally avarlable for release from a reservoir below the maxımum controllable level For power and irrigation reservoirs, this definition is adequate Fol a flood-control reservolr the volume above the spillway crest is excluded from the usable storage although recognized and allowed for in the design of the dam and reservolr For multipurpose reservoirs the volume avalable for release may be dictated by allocations for various uses, but such complications have not been considered in this report

\section{STORAGE RATIO}

The storage ratio is defined as the ratio of usable capacity to the average annual runoff. Assuming average runoff conditions, this ratio represents the time, in years, required to impound a volume of water equal to the usable capacity Conversely, the storage ratio represents the time required to release the usable contents from a reservoir if the average natural rate of flow were maintanned without any inflow The storage ratio concept was first introduced by Hazen (1914) in his analysis of storage capacity required for reservoirs for municipal water supply

To compute the storage ratio it was necessary to estimate the average flow at most dams. Computed storage ratios are reasonably accurate where long-term gaging-station records of flow are avalable at the dam or at any point not too distant upstream or downstream Where such records were unavalable, however, estımates of average flow were generally made on the basis of comparison with other nearby gaged streams Although such estimates may be considerably in error, they may be considered adequate for the purpose of computing the approximate storage ratio, which is generally given to only one or two significant figures No storage ratios are given if the paucity of data precludes even rough approximations of the average runoff or if the storage ratio would not be meaningful, as in the case of an offstream reservoir filled by diversion from the main stream Storage ratios have been omitted for those reservoirs in the Tennessee River basin where the ratio would not be representative owing to other appreciable upstream storage within the same watershed

In many reservons, particularly those on the larger ineis, the total volume of poss ble stolage constitutes only a small fraction of the total ı unoff A storage ratio of 005 yea, or only about 18 days, was chosen as a low er 11mit, and storage iatios less than this amount are shown as $<005$ 
The storage ratios shown are intended only as indicators of the storage capacity of the reservolr as compared with the average flow of the stream and should not be used to estimate annual runoff

USE

The following classifications of reservoir use are shown in table 1
$\mathrm{C}$, conservation
$\mathrm{N}$, navigation
$\mathrm{D}$, desilting
$\mathrm{P}$, power
F, flood control
$R$, recreation
I, irrigation
W, mdustrial
$\mathrm{L}, \log$ driving
$\mathrm{Y}$, low flow augmentation
M, municipal

The uses for multipurpose reser voirs are listed in alphabetıcal order, not in order of importance Only the present use may be indicated for many of the older reservoirs that no longer serve their origmal purpose, for example, some reservoirs originally bult for log driving are now used only tor recreation or have had power generating equipment installed

\section{DATE COMPLETED}

The date completed is the year in which construction of the dam was completed At many dams, storage began during construction, and the beginning of effect of the reservorr on streamflow therefore preceded the completion of the dam, particularly on the larger works Only the latest date of completion is shown for changes that appreciably affected the storage capacity, such as the rasing of the dam or the addition of gates At reservorrs where many changes have been made, the date of only the most significant change is shown Reservorrs that were under construction as of Jan 1, 1963, are footnoted accordingly in table 1

\section{OWNER OR OPERATOR}

The name of the owner or operator of the reservolr is shown in table 1 Generally the owner and operator are the same, however, many western reservorrs that were bult by the Bureau of Reclamation are operated by watel users associations The name of the owner or operator may prove of assistance in obtaining additional data on any particular reservoir included in this compilation 


\section{STORAGE RECORDS PUBLISHED}

If records of storage are published, the name of the publication will be noted in table 1 as follows

PA, US Geological Survey Water-Supply Paper and annual State report entitled "Surface Water Records"

PB, Canada Surface-Water-Supply Paper

PC, State Engineer Report

PD, State Engineer Bulletin

PE, T V A "Operation of Reservorrs"

PF, Pennsylvanıa Dept of Forests and Waters Report

PG, N Y C Dept Water Supply, Gas and Electric Annual Report

PH, Annual Reports of Baltimore Department of Public Works

PI, Shasta River Water-Master Service Area Report

PJ, Pathfinder Irrigation Dist Report

PK, Honolulu Board of Water Supply Report

PL, Corps of Engmeers Report

PM, U S and Mexıco International Boundary and Water Commission Bulletin

PN, Newark Department of Public Affairs, Division of Water Report

PP, Souris River Complation

Storage data for many of the reservoirs listed in this report have been published each water year (Oct. 1 to Sept 30) in Geological Survey water-supply papers Beginning with water year 1961, the annual water-supply papers were replaced with Geological Survey annual State reports entitled "Surface Water Records", the water-supply papers are to be published only as summary reports every 5 years These water-supply papers are publıshed according to "Parts", a State report includes data for that State only Unpublished storage records for many reservoirs are avallable from the owner or operator as shown in table 1 
Table 1 --Reservolrs in the United States completed or under construction as of January 1, 1963, having a usable capac1ty of 5,000 acre-feet or more

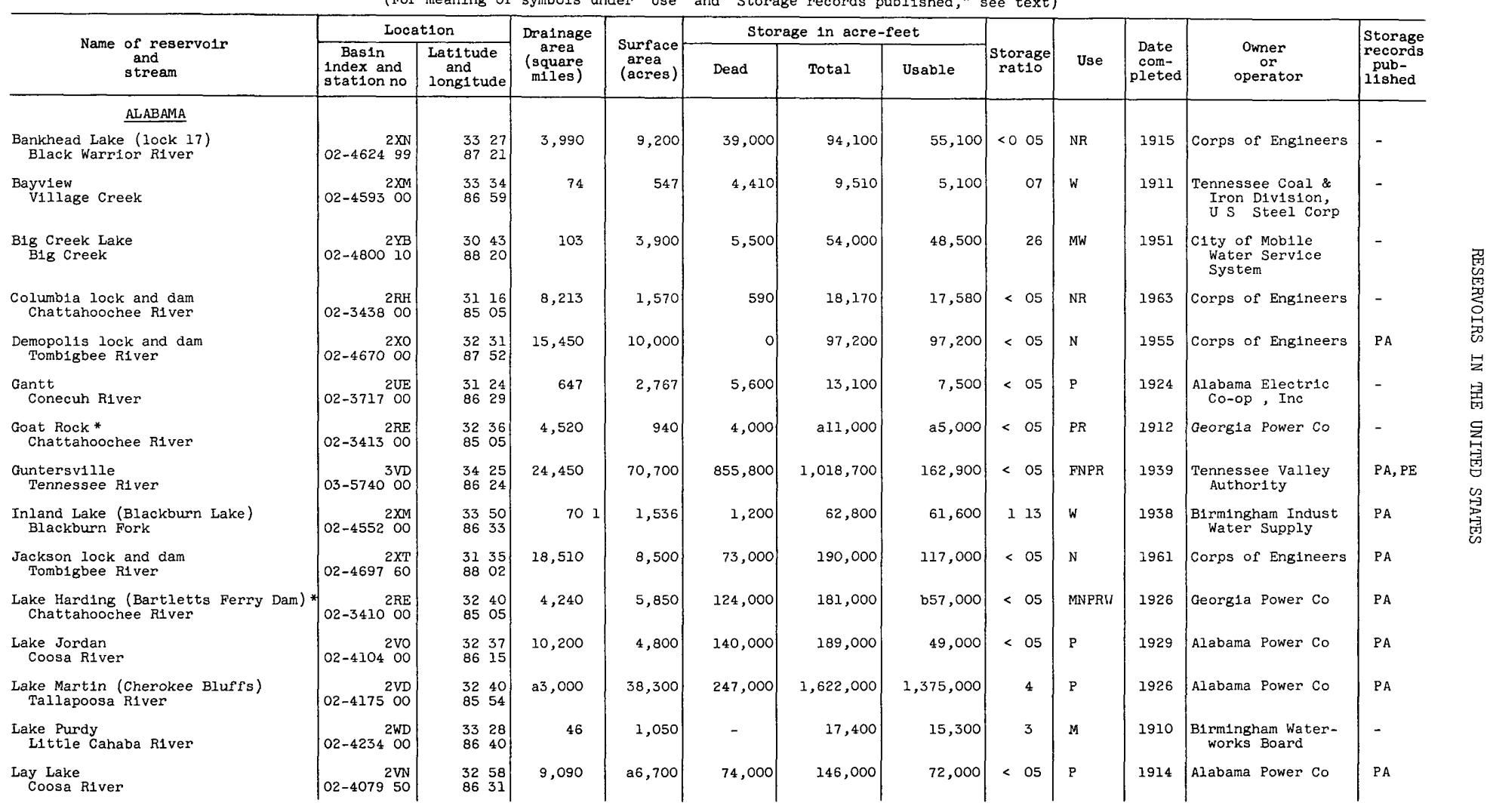


Lew1s Smith
Sipsey Fork

M1tchell Lake

Walter F George
Chat tahoochee River

Warrior lock and dam

Warrior lock and dam
Black Warrior River

Coosa Rive

Wheeler

Tennessee River

Wilson (Muscle Shoals Dam)

ALASKA

Annex Creek
Annex Creek

Annex Cree

Blue Lake
Sawm1ll Creek

Cooper Lake

Cooper Creek

Eklutna Lake
Eklutna Creek

Ketch1kan Lake

Lake Connell
Ward Creek

Purple Lake
Purple Lake Outlet

Salmon Creek
Salmon Creek

Salmon Creek

Upper Silvas Lake

ARIZONA

Apache Lake (Horse Mesa Dam)

Salt RIver

See footnotes at end of table

\begin{tabular}{|c|c|c|c|c|}
\hline $02-4519^{2 \times K} 50$ & $\begin{array}{ll}33 & 56 \\
87 & 06\end{array} \mid$ & 944 & 21,000 & 995,700 \\
\hline (02-4094 $2 \mathrm{VO}$ & $\begin{array}{ll}32 & 48 \\
86 & 26\end{array}$ & 9,830 & 5,800 & 93,000 \\
\hline $02-343240$ & $\begin{array}{ll}31 & 37 \\
85 & 04\end{array}$ & 7,400 & 48,600 & 196,700 \\
\hline $02-466030$ & $\begin{array}{ll}32 & 47 \\
87 & 51\end{array}$ & 5,800 & 7,800 & 4,250 \\
\hline $\begin{array}{r}2 \mathrm{VK} \\
02-3994 \quad 99\end{array}$ & $\begin{array}{ll}34 & 11 \\
85 & 45\end{array}$ & 5,270 & 28,300 & 8,510 \\
\hline $03-5860$ o 00 & $\begin{array}{ll}34 & 48 \\
87 & 23\end{array}$ & 29,590 & 68,300 & 802,900 \\
\hline $03-589000$ & $\begin{array}{ll}34 & 48 \\
87 & 37\end{array}$ & 30,750 & 16,000 & 597,000 \\
\hline $30-0 \overline{4} 1900$ & $\begin{array}{rr}58 & 20 \\
134 & 08\end{array}$ & $\begin{array}{ll}6 & 1\end{array}$ & 262 & 0 \\
\hline $30-087800$ & $\begin{array}{rr}57 & 04 \\
135 & 12\end{array}$ & 370 & 1,215 & 0 \\
\hline $30-2 \overline{590} 00$ & $\begin{array}{rr}60 & 26 \\
149 & 49\end{array}$ & 318 & 2,900 & 97,000 \\
\hline $30-278000$ & $\begin{array}{rr}61 & 24 \\
149 & 09\end{array}$ & 119 & 3,345 & - \\
\hline $30-063800$ & $\begin{array}{rr}55 & 22 \\
131 & 37\end{array}$ & 85 & 580 & 2,020 \\
\hline $30-0 \overline{6} 1800$ & $\begin{array}{rr}55 & 26 \\
131 & 40\end{array}$ & 128 & 220 & 0 \\
\hline $30-057900$ & $\begin{array}{rr}55 & 07 \\
131 & 26\end{array}$ & 68 & 963 & 7,000 \\
\hline $30-051000$ & \begin{tabular}{rr|}
58 & 20 \\
134 & 24
\end{tabular} & $\begin{array}{ll}54 \\
\end{array}$ & 200 & 0 \\
\hline $30-065600$ & $\begin{array}{rr}55 & 23 \\
131 & 31\end{array}$ & $\begin{array}{ll}3 & 4\end{array}$ & 227 & 0 \\
\hline${ }_{-}^{9 N A}$ & $\begin{array}{rr}33 & 36 \\
111 & 21\end{array}$ & 5,935 & 2,656 & 0 \\
\hline
\end{tabular}

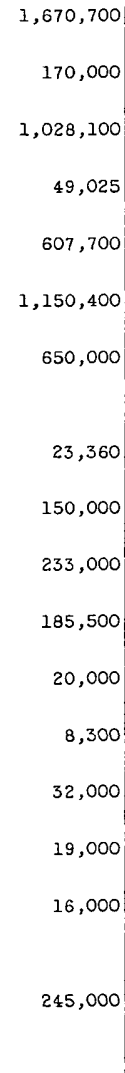

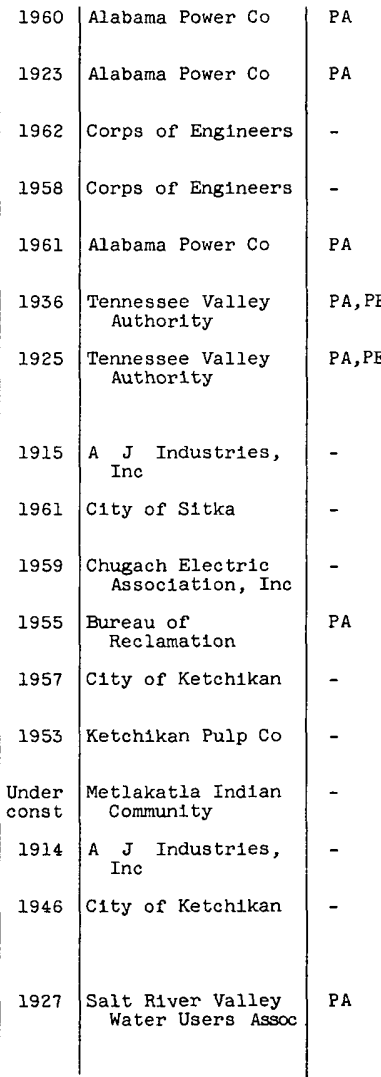


Table 1 --Reservo1rs in the United States completed or under construction as of January 1, 1963, having a usable capac1ty of 5,000 acre-feet or more--Continued [For meaning of symbols under "Use" and "Storage records published," see text]

\begin{tabular}{|c|c|c|c|c|c|c|c|c|c|c|c|c|}
\hline \multirow[b]{2}{*}{$\begin{array}{l}\text { Name of reservolr } \\
\text { and } \\
\text { stream }\end{array}$} & \multicolumn{2}{|c|}{ Location } & \multirow{2}{*}{$\begin{array}{l}\text { Drainage } \\
\text { area } \\
\text { (square } \\
\text { m1les) }\end{array}$} & \multirow[b]{2}{*}{$\begin{array}{c}\text { Surface } \\
\text { area } \\
\text { (acres) }\end{array}$} & \multicolumn{3}{|c|}{ Storage in acre-feet } & \multirow[b]{2}{*}{$\begin{array}{c}\text { Storage } \\
\text { rat1o }\end{array}$} & \multirow[b]{2}{*}{ Use } & \multirow[b]{2}{*}{$\begin{array}{c}\text { Date } \\
\text { com- } \\
\text { pleted }\end{array} \mid$} & \multirow[b]{2}{*}{$\begin{array}{l}\text { Owner } \\
\text { or } \\
\text { operator }\end{array}$} & \multirow{2}{*}{$\begin{array}{l}\text { Storage } \\
\text { records } \\
\text { pub- } \\
11 \text { shed }\end{array}$} \\
\hline & $\begin{array}{l}\text { Basin } \\
\text { 1ndex and } \\
\text { station no }\end{array}$ & $\begin{array}{l}\text { Latitude } \\
\text { and } \\
\text { long1tude }\end{array}$ & & & Dead & Total & Usable & & & & & \\
\hline ARIZONA-ContInued & & & & & & & & & & & & \\
\hline $\begin{array}{l}\text { Bartlett } \\
\text { Verde River }\end{array}$ & $09-509500$ & $\begin{array}{rr}33 & 49 \\
111 & 38\end{array}$ & 6,185 & 2,768 & 0 & 179,500 & 179,500 & 04 & FIMR & 1939 & $\begin{array}{l}\text { Salt River Valley } \\
\text { Water Users Assoc. }\end{array}$ & PA \\
\hline $\begin{array}{l}\text { Big Lake } \\
\text { North Fork of East Fork of Black } \\
\text { River tributary }\end{array}$ & $-9 \mathrm{NA}$ & $\begin{array}{rr}33 & 53 \\
109 & 25\end{array}$ & 31 & 570 & 0 & 12,200 & 12,200 & - & $\mathrm{R}$ & 1953 & $\begin{array}{l}\text { Arizona Game and } \\
\text { Fish Department }\end{array}$ & - \\
\hline $\begin{array}{l}\text { Canyon Lake (Mormon Flat Dam) } \\
\text { Salt River }\end{array}$ & $-9 N A$ & $\begin{array}{rr}33 & 33 \\
111 & 26\end{array}$ & 6,094 & 950 & 0 & 57,900 & 57,900 & 08 & IP & 1925 & $\begin{array}{l}\text { Salt River Valley } \\
\text { Water Users Assoc }\end{array}$ & PA \\
\hline $\begin{array}{l}\text { Cave Creek } \\
\text { Cave Creek }\end{array}$ & $09-512350$ & $\begin{array}{rr}33 & 44 \\
112 & 03\end{array}$ & 161 & 680 & 0 & 11,000 & 11,000 & - & $\mathrm{F}$ & 1923 & $\begin{array}{l}\text { Salt River Valley } \\
\text { Water Users Assoc }\end{array}$ & - \\
\hline $\begin{array}{l}\text { Havasu Lake (Parker Dam) } \\
\text { Colorado River }\end{array}$ & 9LB & $\begin{array}{rr}34 & 18 \\
114 & 08\end{array}$ & 178,800 & 20,390 & - & 619,400 & 180,000 & - & FIMPR & 1938 & $\begin{array}{l}\text { Bureau of } \\
\text { Reclamation }\end{array}$ & PA \\
\hline $\begin{array}{l}\text { Horseshoe } \\
\text { Verde River }\end{array}$ & 09-5095 90 & $\begin{array}{rr}33 & 59 \\
111 & 43\end{array}$ & 5,991 & 2,719 & 0 & 142,800 & 142,800 & 4 & FIMPR & 1945 & $\begin{array}{l}\text { Salt River Valley } \\
\text { Water Users Assoc }\end{array}$ & PA \\
\hline $\begin{array}{l}\text { Lake Mary (Lower) } \\
\text { Walnut Creek }\end{array}$ & $09-400900$ & $\begin{array}{rr}35 & 07 \\
111 & 35\end{array}$ & 119 & 838 & 0 & 7,978 & 7,978 & - & MR & 1912 & City of Flagstaff & - \\
\hline $\begin{array}{l}\text { Lake Mary (Upper) } \\
\text { Walnut Creek }\end{array}$ & $09-400850$ & $\begin{array}{rr}35 & 07 \\
111 & 35\end{array}$ & 84 & 1,058 & 0 & 16,576 & 16,576 & - & MR & 1941 & City of Flagstaff & - \\
\hline $\begin{array}{l}\text { Lake Mead (Hoover Dam) * } \\
\text { Colorado River }\end{array}$ & $09-4210^{9 L B}$ & $\begin{array}{rr}36 & 01 \\
114 & 44\end{array}$ & 167,800 & 157,736 & $2,620,000$ & $29,827,000$ & $27,207,000$ & 22 & FIMNP & 1936 & $\begin{array}{l}\text { Bureau of } \\
\text { Reclamation }\end{array}$ & $\mathrm{PA}$ \\
\hline $\begin{array}{l}\text { Lake Mohave (Davis Dam)* } \\
\text { Colorado River }\end{array}$ & $\begin{array}{r}9 L B \\
09-422500\end{array}$ & $\begin{array}{rr}35 & 12 \\
114 & 34\end{array}$ & 169,300 & 28,192 & 8,530 & $1,818,300$ & $1,809,800$ & - & IP & 1949 & $\begin{array}{l}\text { Bureau of } \\
\text { Reclamation }\end{array}$ & PA \\
\hline $\begin{array}{l}\text { Lake Pleasant } \\
\text { Agua Fria River }\end{array}$ & 9ND & $\begin{array}{rr}33 & 51 \\
112 & 16\end{array}$ & 1,459 & 3,585 & 0 & 163,800 & 163,800 & 26 & I & 1927 & $\begin{array}{l}\text { Maricopa County } \\
\text { Municipal Water } \\
\text { Conserv Dist No } 1\end{array}$ & PA \\
\hline $\begin{array}{l}\text { Lake Powell (Glen Canyon Dam) } \\
\text { Colorado River }\end{array}$ & $09-379900$ & $\begin{array}{rr}36 & 57 \\
111 & 29\end{array}$ & $a 107,700$ & 161,390 & $1,998,000$ & $27,000,000$ & $20,876,000$ & 16 & FIPR & 1963 & $\begin{array}{l}\text { Bureau of } \\
\text { Reclamation }\end{array}$ & PA \\
\hline $\begin{array}{l}\text { Lake Show Low (Jaques Dam) } \\
\text { Show Low Creek }\end{array}$ & $\begin{array}{r}9 \mathrm{JA} \\
09-3910 \quad 00\end{array}$ & $\begin{array}{rr}34 & 12 \\
110 & 00\end{array}$ & 672 & 194 & 1,070 & 6,176 & 5,106 & 9 & RW & 1953 & Phelps Dodge Corp & PA \\
\hline $\begin{array}{l}\text { Lyman } \\
\text { Little Colorado River }\end{array}$ & O9-3845 90 & $\begin{array}{r}3422 \\
10923\end{array}$ & 790 & 2,470 & 0 & 31,390 & 31,390 & 22 & I & 1949 & $\begin{array}{l}\text { Lyman Irrigation } \\
\text { District }\end{array}$ & PA \\
\hline $\begin{array}{l}\text { Many Farms } \\
\text { Chinle Wash } \mathrm{c}\end{array}$ & $\begin{array}{r}9 \mathrm{GH} \\
09-3790 \quad 40\end{array}$ & $\begin{array}{rl}36 & 21 \\
109 & 36\end{array}$ & - & 1,770 & 0 & 22,500 & 22,500 & - & $I$ & 1939 & $\begin{array}{l}\text { Bureau of Indian } \\
\text { Affairs }\end{array}$ & - \\
\hline
\end{tabular}




Pailted Rock
Gila River
Picacho
McClellan Wash c/
Roosevelt
Salt River
Saguaro Lake (Stewart Mountain Dam)
Salt River
San Carlos (Coolidge Dam)
Gila River
Trilby Wash detention basin
(McMicken Dam)
Trilby Wash and other washes
Whitlow Ranch
Queen Creek
Bear Creek Lake
Bear Creek
Blue Mountaln
Pet1t Jean Creek
Bull Shoals
White River
Greers Ferry
Little Red River
Lake Catherine (Remmel Dam)
Ouachita River
Lake Fort Smith
Frog Bayou
Lake Greeson (Narrows Dam)
Little Missouri River
Lake Hamilton (Carpenter Dam)
Ouachita River
Lake Ouachita
Ouachita River
Lake Sheppard Springs
Frog Bayou
Lake Winona
Alum Fork
See footnotes at end of table

\begin{tabular}{|c|c|c|c|}
\hline O9-5197 $9 \mathrm{MF}$ & $\begin{array}{r}33 \text { O4 } \\
113 \text { O1 }\end{array}$ & a50, 900 & 53,200 \\
\hline${ }_{-} \quad 9 M E$ & $\begin{array}{r}3252 \\
11129\end{array}$ & - & 2,000 \\
\hline$-9 \mathrm{NA}$ & $\begin{array}{rr}33 & 40 \\
111 & 10\end{array}$ & 5,824 & 17,315 \\
\hline 09-5010 90 & $\begin{array}{r}3334 \\
11132\end{array}$ & 6,211 & 1,26 \\
\hline $\begin{array}{r}9 M C \\
09-4690 \text { OC }\end{array}$ & $\begin{array}{r}3310 \\
11032\end{array}$ & 12,886 & 19,58 \\
\hline 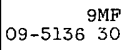 & $\begin{array}{r}3341 \\
11225\end{array}$ & a225 & 2,230 \\
\hline $09-4785 \stackrel{9 M F}{10}$ & $\begin{array}{rl}33 & 18 \\
111 & 16\end{array}$ & 144 & 8 \\
\hline$-7 \mathrm{GE}$ & $\begin{array}{ll}34 & 43 \\
90 & 42\end{array}$ & 57 & 5 \\
\hline $07-2590^{7 \mathrm{FB}}$ & $\begin{array}{ll}35 & 06 \\
93 & 39\end{array}$ & 488 & 11,00 \\
\hline $07-0545$ $7 \mathrm{HA}$ & $\begin{array}{ll}36 & 22 \\
92 & 34\end{array}$ & 6,036 & 71,2 \\
\hline $07-0759 \stackrel{7 \mathrm{HE}}{00}$ & $\begin{array}{ll}35 & 31 \\
91 & 52\end{array}$ & 1,146 & 40,50 \\
\hline $07-3590^{7 \mathrm{JA}} 00$ & $\begin{array}{ll}34 & 26 \\
92 & 54\end{array}$ & 1,516 & 1,94 \\
\hline$-{ }^{7 \mathrm{FB}}$ & $\begin{array}{ll}35 & 40 \\
94 & 09\end{array}$ & 744 & 4 \\
\hline $\begin{array}{r}7 J B \\
07-360500\end{array}$ & $\begin{array}{ll}34 & 09 \\
93 & 43\end{array}$ & 237 & 9,80 \\
\hline $07-3585{ }^{7 \mathrm{JA}} 00$ & $\begin{array}{ll}34 & 26 \\
93 & 02\end{array}$ & 1,441 & 7,15 \\
\hline $\begin{array}{r}7 \mathrm{JA} \\
07-3575 \text { OO }\end{array}$ & $\begin{array}{ll}34 & 34 \\
93 & 12\end{array}$ & 1,105 & 40,10 \\
\hline $7 F^{\prime} B$ & $\begin{array}{ll}35 & 41 \\
94 & 07\end{array}$ & 65 & 7 \\
\hline${ }_{-}^{7 J D}$ & $\begin{array}{ll}34 & 48 \\
92 & 51\end{array}$ & 435 & 2,240 \\
\hline
\end{tabular}
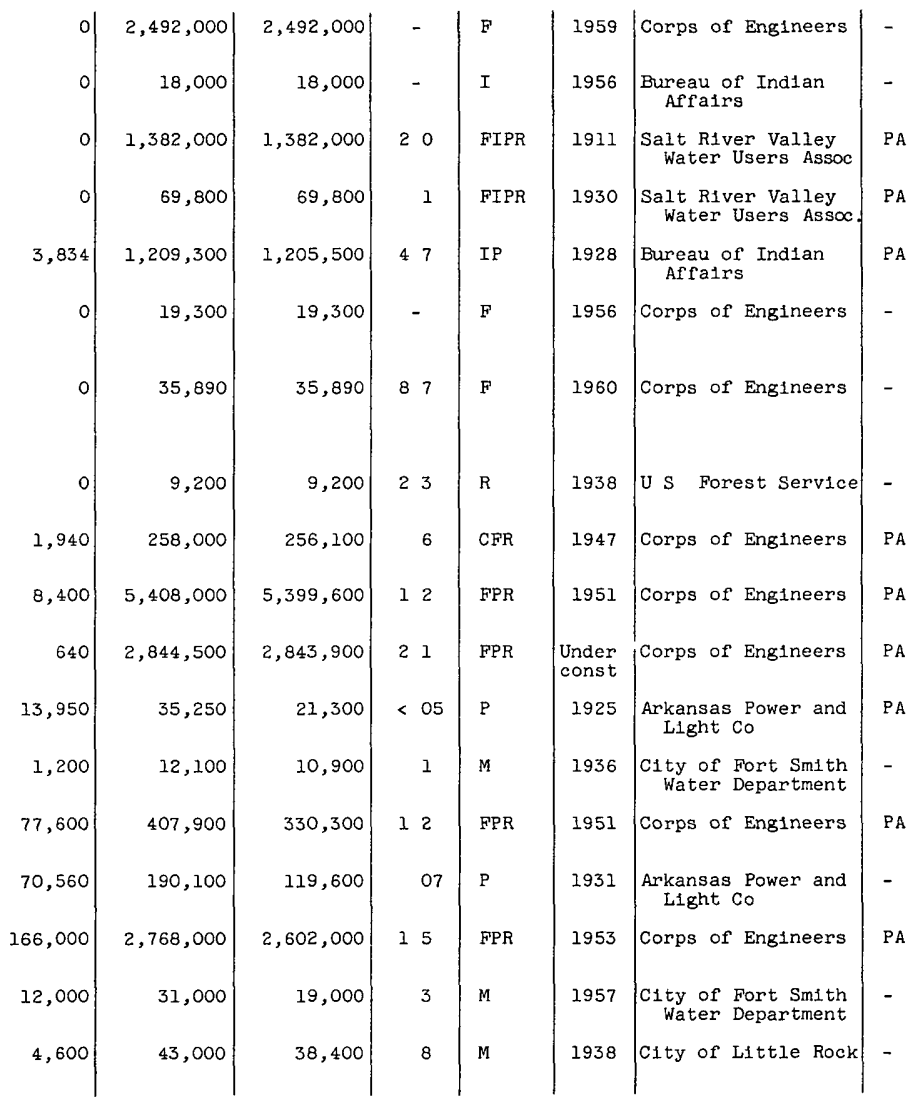
Table 1 --Reservoirs in the United States completed or under construction as of January 1, 1963, having a usable capacity of 5,000 acre-feet or more--Cont1nued

\begin{tabular}{|c|c|c|c|c|c|c|c|c|c|c|c|c|}
\hline \multirow[b]{2}{*}{$\begin{array}{l}\text { Name of reservolr } \\
\text { and } \\
\text { stream }\end{array}$} & \multicolumn{2}{|c|}{ Location } & \multirow{2}{*}{$\begin{array}{l}\text { Drainage } \\
\text { area } \\
\text { (square } \\
\text { m1les) }\end{array}$} & \multirow[b]{2}{*}{$\begin{array}{c}\text { Surface } \\
\text { area } \\
\text { (acres) }\end{array}$} & \multicolumn{3}{|c|}{ Storage in acre-feet } & \multirow[b]{2}{*}{$\begin{array}{c}\text { Storage } \\
\text { rat10 }\end{array}$} & \multirow[b]{2}{*}{ Use } & \multirow[b]{2}{*}{$\begin{array}{l}\text { Date } \\
\text { com- } \\
\text { pleted }\end{array}$} & \multirow[b]{2}{*}{$\begin{array}{l}\text { Owner } \\
\text { or } \\
\text { operator }\end{array}$} & \multirow{2}{*}{$\begin{array}{l}\text { Storage } \\
\text { records } \\
\text { pub- } \\
\text { I1shed }\end{array}$} \\
\hline & \begin{tabular}{|c|} 
Basin \\
index and \\
station no
\end{tabular} & $\begin{array}{l}\text { Lat1 tude } \\
\text { and } \\
\text { long1 tude }\end{array}$ & & & Dead & Total & Usable & & & & & \\
\hline ARKANSAS--Cont1nued & & & & & & & & & & & & \\
\hline $\begin{array}{l}\text { Maumelle Lake } \\
\text { Maumelle River }\end{array}$ & $-7 \mathrm{FC}$ & $\begin{array}{ll}34 & 51 \\
92 & 29\end{array}$ & 120 & 8,900 & 9,000 & 208,000 & 199,000 & 27 & M & 1957 & C1ty of Little Rock & - \\
\hline $\begin{array}{l}\text { Nimrod } \\
\text { Fourche La Fave River }\end{array}$ & $07-2620^{7 \mathrm{FC}}$ & $\begin{array}{ll}34 & 57 \\
93 & 10\end{array}$ & 680 & 18,300 & 900 & 336,000 & 335,100 & 5 & $\mathrm{CFR}$ & 1942 & Corps of Engineers & PA \\
\hline $\begin{array}{l}\text { Norfork } \\
\text { North Fork River }\end{array}$ & $07-059500$ & $\begin{array}{ll}36 & 15 \\
92 & 14\end{array}$ & 1,806 & 30,700 & 2,500 & $1,983,000$ & $1,980,500$ & 14 & CFPR & 1950 & Corps of Engineers & $\mathrm{PA}$ \\
\hline $\begin{array}{l}\text { Storm Creek Lake } \\
\text { Storm Creek }\end{array}$ & $-7 G E$ & $\begin{array}{ll}34 & 36 \\
90 & 37\end{array}$ & 93 & 510 & 아 & 7,650 & 7,650 & 13 & $\mathrm{R}$ & 1939 & U S Forest Service & - \\
\hline \multicolumn{13}{|l|}{ CALIFORNIA } \\
\hline $\begin{array}{l}\text { Alpine } \\
\text { Lagun1 tas Creek }\end{array}$ & $-^{21 E L}$ & $\begin{array}{rr}37 & 57 \\
122 & 38\end{array}$ & 105 & 224 & - & 9,210 & - & - & M & 1917 & $\begin{array}{l}\text { Marin Municipal } \\
\text { Water District }\end{array}$ & - \\
\hline $\begin{array}{l}\text { Anderson Lake } \\
\text { Coyote Creek }\end{array}$ & $\begin{array}{r}11 G 0 \\
11-169920\end{array}$ & $\begin{array}{rr}37 & 10 \\
121 & 38\end{array}$ & 194 & 980 & 0 & 91,310 & 91,310 & 18 & I & 1950 & $\begin{array}{c}\text { Santa Clara County } \\
\text { WaterConserv Dist }\end{array}$ & $\mathrm{PA}$ \\
\hline $\begin{array}{l}\text { Barrett } \\
\text { Cottonwood Creek }\end{array}$ & $-11 G \mathrm{~A}$ & $\begin{array}{rr}32 & 41 \\
116 & 40\end{array}$ & 250 & 794 & 719 & $\mathrm{~d} 44,760$ & $\mathrm{~d} 44,040$ & - & M & 1922 & City of San Diego & PA \\
\hline $\begin{array}{l}\text { Bass Lake (Crane Valley) } \\
\text { North Fork W1liow Creek }\end{array}$ & $\begin{array}{r}11 \mathrm{FG} \\
11-243400\end{array}$ & $\begin{array}{rr}37 & 17 \\
119 & 32\end{array}$ & 505 & 1,165 & 300 & 45,400 & 45,100 & 10 & $P$ & 1901 & $\begin{array}{l}\text { Pacific Gas and } \\
\text { Electric Co }\end{array}$ & PA \\
\hline $\begin{array}{l}\text { Bear } \\
\text { Bear River }\end{array}$ & $\begin{array}{r}11 \mathrm{FJ} \\
11-260217\end{array}$ & $\begin{array}{rr}37 & 22 \\
120 & 14\end{array}$ & 721 & 265 & 0 & 7,700 & 7,700 & - & $F$ & 1950 & Corps of Engineers & - \\
\hline $\begin{array}{l}\text { Bear River } \\
\text { Bear River }\end{array}$ & $\begin{array}{r}11 \mathrm{DL} \\
11-315400\end{array}$ & $\begin{array}{rr}38 & 33 \\
120 & 13\end{array}$ & 280 & 169 & - & 6,760 & - & - & $P$ & 1900 & $\begin{array}{l}\text { Pacific Gas and } \\
\text { Electric Co }\end{array}$ & - \\
\hline $\begin{array}{l}\text { Beardsley Lake } \\
\text { M1ddle Fork Stan1slaus }\end{array}$ & $\begin{array}{r}11 \mathrm{FM} \\
11-292800\end{array}$ & $\begin{array}{rr}38 & 12 \\
120 & 05\end{array}$ & 303 & 650 & 0 & 98,300 & 98,300 & 7 & $P$ & 1957 & $\begin{array}{l}\text { Oakdale-South San } \\
\text { Joaquin Irrig Dists }\end{array}$ & PA \\
\hline $\begin{array}{l}\text { Big Bear Lake } \\
\text { Bear Creek }\end{array}$ & $11-049000$ & $\begin{array}{rr}34 & 14 \\
116 & 59\end{array}$ & 38 & 2,600 & 0 & 72,200 & 72,200 & 52 & I & 1884 & $\begin{array}{l}\text { Bear Valley Mutual } \\
\text { Water Co, Ltd }\end{array}$ & $P A$ \\
\hline $\begin{array}{l}\text { Big Creek No } 7 \text { (Redinger Lake) } \\
\text { San Juaquin R1ver }\end{array}$ & $-11 \mathrm{PG}$ & $\begin{array}{rr}37 & 09 \\
119 & 26\end{array}$ & 1,292 & 465 & 8,914 & 35,033 & 26,119 & $<05$ & $P$ & 1951 & $\begin{array}{l}\text { Southern Cal1fornia } \\
\text { Edison Co }\end{array}$ & - \\
\hline $\begin{array}{l}\text { Big Dobe-North } \\
\text { Rattlesnake Creek }\end{array}$ & $-^{11 B D}$ & $\begin{array}{rr}41 & 40 \\
120 & 30\end{array}$ & 1,600 & 1,600 & - & 6,530 & - & - & $I$ & 2912 & Dav1d $J$ Bayne & - \\
\hline $\begin{array}{l}\text { Big Dry Creek } \\
\text { Big Dry Creek }\end{array}$ & $-^{11 \mathrm{FH}}$ & $\begin{array}{rr}36 & 53 \\
119 & 42\end{array} \mid$ & 860 & 1,530 & 0 & 16,250 & 16,250 & - & F & 1948 & Corps of Engineers & - \\
\hline
\end{tabular}


Big Sage

Rattlesnake Creek

Black Butte

Stony Creak

Blue Lake (Lower)
Blue Creek

Blue Lake (Upper)

Boca
L1ttle Truckee River

Bouquet

Bouquet Creek

Bowman Lake
Canyon Cree

Bridgeport

East Walker RIver

Bucks
Bucks Creek

Bucks Lake
Bucks Creek

Buena Vista Lake

Kern R1ver

Bullards Bar

North Fork Yuba River

Burns
Burns Creek

Butt Valley

Calaveras

Calaveras Creek

Calero

Arroyo Calero

Camanche

\section{Mokelumne}

Carbon Canyon

Carbon Canyon Creek

Casitas

Creek

See footnotes at end of table

\begin{tabular}{|c|c|c|c|}
\hline $\mid \begin{array}{r}11-3481 \mathrm{BD} \\
\mid\end{array}$ & $\begin{array}{r}4135 \\
12038\end{array}$ & 107 & 5,270 \\
\hline${ }_{-}^{I I B N}$ & $\begin{array}{rr}39 & 48 \\
122 & 15\end{array}$ & 741 & 4,560 \\
\hline $\begin{array}{r}11 D L \\
11-313475\end{array}$ & $\begin{array}{rr}38 & 37 \\
119 & 56\end{array}$ & 48 & 157 \\
\hline $\begin{array}{r}11 \mathrm{DL} \\
11-313470\end{array}$ & $\begin{array}{r}3837 \\
11956\end{array}$ & 27 & 354 \\
\hline $\begin{array}{r}10 \mathrm{BB} \\
10-344490\end{array}$ & $\begin{array}{r}3923 \\
12006\end{array}$ & 172 & 980 \\
\hline $\begin{array}{r}11 \mathrm{GF} \\
11-107820\end{array}$ & $\begin{array}{r}34 \quad 35 \\
118 \quad 24\end{array}$ & 136 & 628 \\
\hline $\begin{array}{r}11 \mathrm{CK} \\
11-415500\end{array}$ & $\begin{array}{r}3927 \\
12039\end{array}$ & 294 & 825 \\
\hline $\begin{array}{r}10 \mathrm{OEB} \\
11-292500\end{array}$ & $\begin{array}{rr}38 & 20 \\
119 & 13\end{array}$ & 362 & 3,000 \\
\hline$-^{11 C C}$ & $\begin{array}{rr}39 & 54 \\
121 & 13\end{array}$ & 306 & 136 \\
\hline $\begin{array}{r}11 \mathrm{CC} \\
11-403500\end{array}$ & $\begin{array}{r}3954 \\
121 \quad 12\end{array}$ & 286 & 1,827 \\
\hline $\begin{array}{r}11 \mathrm{FD} \\
11-185200\end{array}$ & $\begin{array}{r}3515 \\
11918\end{array}$ & 373 & 23,270 \\
\hline $\begin{array}{r}11 \mathrm{CG} \\
11-413440\end{array}$ & $\begin{array}{r}3924 \\
12108\end{array}$ & 486 & 515 \\
\hline $\begin{array}{r}11 \mathrm{FJ} \\
11-260230\end{array}$ & $\begin{array}{r}3723 \\
12016\end{array}$ & 738 & 670 \\
\hline $11-401050$ & $\begin{array}{rr}40 & 07 \\
121 & 09\end{array}$ & 750 & 1,600 \\
\hline$-^{11 G P}$ & $\begin{array}{r}3729 \\
12149\end{array}$ & - & 1,450 \\
\hline $\begin{array}{r}1160 \\
11-166740\end{array}$ & $\begin{array}{r}3711 \\
12147\end{array}$ & 70 & 325 \\
\hline $2^{11 D K}$ & $\begin{array}{r}3815 \\
12100\end{array}$ & 618 & 7,699 \\
\hline$-^{11 G E}$ & $\begin{array}{r}3355 \\
11751\end{array}$ & 193 & 237 \\
\hline $\begin{array}{r}11 \mathrm{GF} \\
11-117900\end{array}$ & $\begin{array}{r}3422 \\
11920\end{array}$ & 39 & 2,710 \\
\hline
\end{tabular}

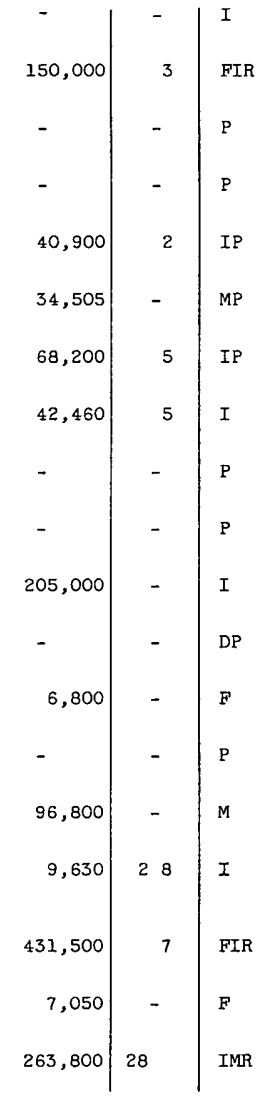

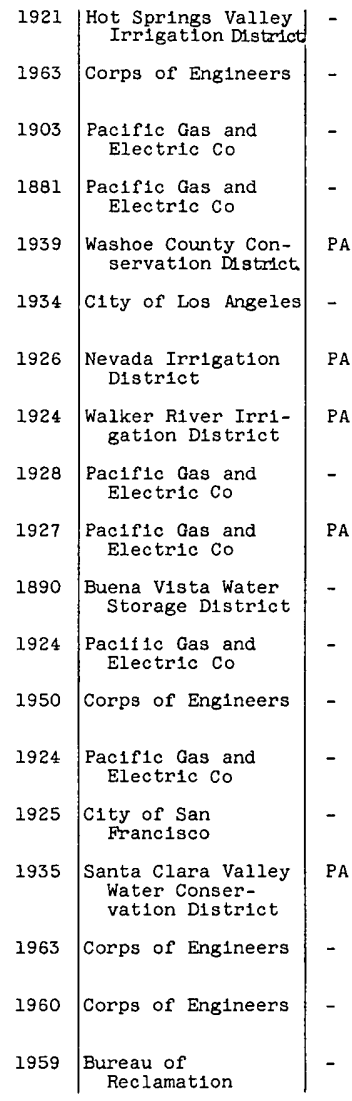



[For meaning of symbols under "Use" and "Storage records published," see text]

\begin{tabular}{|c|c|c|c|c|c|c|c|c|c|c|c|c|}
\hline \multirow[b]{2}{*}{$\begin{array}{l}\text { Name of reservo1r } \\
\text { and } \\
\text { stream }\end{array}$} & \multicolumn{2}{|c|}{ Location } & \multirow{2}{*}{$\begin{array}{l}\text { Drainage } \\
\text { area } \\
\text { (square } \\
\text { m1les) }\end{array}$} & \multirow[b]{2}{*}{$\begin{array}{l}\text { Surface } \\
\text { area } \\
\text { (acres) }\end{array}$} & \multicolumn{3}{|c|}{ Storage in acre-feet } & \multirow[b]{2}{*}{$\begin{array}{c}\text { Storage } \\
\text { rat1o }\end{array}$} & \multirow[b]{2}{*}{ Use } & \multirow[b]{2}{*}{$\begin{array}{c}\text { Date } \\
\text { com- } \\
\text { pleted }\end{array}$} & \multirow[b]{2}{*}{$\begin{array}{l}\text { Owner } \\
\text { or } \\
\text { operator }\end{array}$} & \multirow{2}{*}{$\begin{array}{l}\text { Storage } \\
\text { records } \\
\text { pub- } \\
\text { l1shed }\end{array}$} \\
\hline & \begin{tabular}{|c|} 
Basin \\
1ndex and \\
station no \\
\end{tabular} & $\begin{array}{c}\text { Lat1 tude } \\
\text { and } \\
\text { long1tude }\end{array}$ & & & Dead & Total & Usable & & & & & \\
\hline CALIFORNIA--Continued & & & & & & & & & & & & \\
\hline $\begin{array}{l}\text { Chabot (Lower San Leandro Dam) } \\
\text { San Leandro Creek }\end{array}$ & $11-181200$ & $\begin{array}{rr}37 & 44 \\
122 & 07\end{array}$ & 43 & 315 & - & - & - & - & M & 1875 & \begin{tabular}{|} 
East Bay Municipal \\
Utility District
\end{tabular} & - \\
\hline $\begin{array}{l}\text { Chatsworth } \\
\text { Tr1butary to Los Angeles R1ver }\end{array}$ & $-^{11 G E}$ & $\begin{array}{rr}34 & 14 \\
118 & 38\end{array}$ & $\begin{array}{lll}5 & 4\end{array}$ & 607 & 0 & 9,886 & 9,886 & - & M & 1918 & C1ty of Los Angeles & - \\
\hline $\begin{array}{l}\text { Cherry Lake } \\
\text { Cherry Creek }\end{array}$ & $11-277200$ & $\begin{array}{rr}37 & 58 \\
119 & 54\end{array}$ & 117 & 1,765 & 20 & 268,200 & 268,200 & 10 & FIMP & 1956 & $\begin{array}{r}\text { C1ty of San } \\
\text { Franc1sco }\end{array}$ & PA \\
\hline $\begin{array}{l}\text { Chesbro } \\
\text { Llagas Creek }\end{array}$ & $\begin{array}{r}11 G 0 \\
11-153480\end{array}$ & $\begin{array}{rr}37 & 07 \\
121 & 42\end{array}$ & 194 & 265 & o & 7,500 & 7,500 & 7 & FI & 1955 & $\begin{array}{l}\text { Santa Clara Water } \\
\text { Conserv District }\end{array}$ & PA \\
\hline $\begin{array}{l}\text { Chet Harrit } \\
\text { Qua11 Canyon Creek }\end{array}$ & $-11 \mathrm{GA}$ & $\begin{array}{rr}32 & 51 \\
216 & 53\end{array}$ & 17 & 181 & 750 & 10,500 & 9,750 & - & M & $\begin{array}{l}\text { Under } \\
\text { Const }\end{array}$ & $\left\{\begin{array}{l}\text { Helix Irrigation } \\
\text { District }\end{array}\right.$ & - \\
\hline $\begin{array}{l}\text { Clear Lake } \\
\text { Cache Creek }\end{array}$ & $\begin{array}{r}11 \mathrm{DB} \\
11-4500 \quad 00\end{array}$ & $\begin{array}{rr}39 & 03 \\
122 & 55\end{array}$ & 528 & 43,000 & - & - & 319,000 & 14 & IR & 1915 & $\begin{array}{c}\text { Clear Lake Water } \\
\text { Co }\end{array}$ & PA \\
\hline $\begin{array}{l}\text { Clear Lake } \\
\text { Lost RIver }\end{array}$ & $\begin{array}{r}11 \mathrm{AC} \\
11-483010\end{array}$ & $\begin{array}{rr}41 & 52 \\
121 & 10\end{array}$ & 550 & 24,800 & 13,450 & d526,770 & d513,320 & 21 & $\mathrm{CFI}$ & 1910 & $\begin{array}{l}\text { Bureau of } \\
\text { Reclamation }\end{array}$ & - \\
\hline $\begin{array}{l}\text { Cogswell (formerly San Gabriel No 2) } \\
\text { West Fork San Gabriel R1ver }\end{array}$ & $11-080850$ & $\begin{array}{rr}34 & 15 \\
117 & 58\end{array}$ & 392 & 160 & 35 & 10,630 & 10,595 & 2 & $\mathrm{CF}$ & 1935 & $\begin{array}{l}\text { Los Angeles County } \\
\text { Flood Control D1st }\end{array}$ & - \\
\hline $\begin{array}{l}\text { Combie (Van Ge1sen) } \\
\text { Bear R1ver }\end{array}$ & $-11 C L$ & $\begin{array}{rr}39 & 01 \\
121 & 02\end{array}$ & 130 & 360 & 0 & d7, 840 & d7, 840 & $<05$ & I & 1928 & $\begin{array}{l}\text { Nevada Irrigation } \\
\text { District }\end{array}$ & - \\
\hline $\begin{array}{l}\text { Copco No l } \\
\text { Klamath RIver }\end{array}$ & $-11 \mathrm{AE}$ & $\begin{array}{rr}41 & 59 \\
122 & 20\end{array}$ & 4,300 & 1,000 & b72,000 & 77,000 & ab 5,000 & $<05$ & $P$ & 1917 & $\begin{array}{l}\text { California-Oregon } \\
\text { Power Co }\end{array}$ & - \\
\hline $\begin{array}{l}\text { Copco No } 2 \\
\text { Klamath R1ver }\end{array}$ & $-11 \mathrm{AE}$ & $\overline{-}$ & 7,975 & 825 & - & 58,000 & - & - & $\mathrm{PR}$ & $\begin{array}{l}\text { Under } \\
\text { const }\end{array}$ & $\begin{array}{l}\text { Paclfic Power and } \\
\text { Light Co }\end{array}$ & - \\
\hline $\begin{array}{l}\text { Copper Basin (on Colorado aqueduct) } \\
\text { Copper Wash }\end{array}$ & $-9 L B$ & $\begin{array}{rr}34 & 17 \\
114 & 14\end{array}$ & 82 & 427 & 16,860 & 22,000 & 5,140 & - & M & 1938 & $\begin{array}{l}\text { Metropolitan Water } \\
\text { District of South- } \\
\text { ern California }\end{array}$ & - \\
\hline $\begin{array}{l}\text { Courtright } \\
\text { Helms Creek }\end{array}$ & $\begin{array}{r}11 \mathrm{FE} \\
11-214550\end{array}$ & $\begin{array}{rr}37 & 05 \\
118 & 58\end{array}$ & 397 & 1,621 & 0 & 129,900 & 129,900 & - & $\mathrm{P}$ & 1958 & $\begin{array}{l}\text { Pacific Gas and } \\
\text { Electric Co }\end{array}$ & PA \\
\hline $\begin{array}{l}\text { Coyote } \\
\text { Coyote Creek }\end{array}$ & $\begin{array}{r}11 G 0 \\
11-169850\end{array}$ & \begin{tabular}{rr|}
37 & 07 \\
121 & 33
\end{tabular} & 120 & 688 & 0 & 24,510 & 24,510 & 5 & I & 1936 & $\begin{array}{c}\text { Santa Clara Valley } \\
\text { Water Conser- } \\
\text { vation District }\end{array}$ & PA \\
\hline $\begin{array}{l}\text { Coyote Flat } \\
\text { Coyote Creek }\end{array}$ & $-^{11 B D}$ & $\begin{array}{rr}40 & 54 \\
120 & 59\end{array}$ & 30 & 293 & - & - & - & - & I & 1928 & T E Connolly & - \\
\hline
\end{tabular}


Cuyamaca

Doulder creek

Crystal Springs (Lower)
San Mateo Creek

Dallas-Warner
Tuolumne River $c$ f

Don Pedro

Tuolumne River

Donnell Lake

Middle Fork Stanislaus River

Donner Lake

Donner Creek

Dorr1s

Stookd1ll slough

Dwinnel

Shasta River

East Park

Little Stony Creek

El Capitan

Diego River

Encino

Enc1no Creek

Englebright

Falrmont

Los Angeles aqueduct $c$ f

Fallen Leaf Lake

Taylor creake

Taylor Creek
Taylen Lake

Farmington
Littiejohn Creek

Florence Lake

South Fork San Joaquin R1ver

See footnotes at end of table

\begin{tabular}{|c|c|c|c|c|c|}
\hline$-^{11 \mathrm{GA}}$ & $\begin{array}{rr}32 & 59 \\
116 & 35\end{array}$ & 12 이 & 930 & - 이 & 11,540 \\
\hline $\begin{array}{r}11 \mathrm{GP} \\
11-162750\end{array}$ & $\begin{array}{rr}37 & 31 \\
122 & 22\end{array}$ & 25 & 1,492 & - & - \\
\hline $\begin{array}{r}11 F N \\
11-289200\end{array}$ & $\begin{array}{rr}37 & 39 \\
120 & 41\end{array}$ & - & 3,800 & 2,000 & 27,000 \\
\hline $\begin{array}{r}11 \mathrm{FN} \\
11-287500\end{array}$ & $\begin{array}{rr}37 & 43 \\
120 & 24\end{array}$ & 1,539 & 3,100 & b30,000 & 290,400 \\
\hline $\begin{array}{r}11 F M \\
11-292600\end{array}$ & $\begin{array}{r}3820 \\
11958\end{array}$ & 266 & 430 & 2,150 & 64,740 \\
\hline$-{ }^{1 O B B}$ & $\begin{array}{rr}39 & 19 \\
120 & 12\end{array}$ & 13 & 960 & - & - \\
\hline$-^{11 \mathrm{BB}}$ & $\begin{array}{rr}41 & 29 \\
120 & 28\end{array}$ & 6 & 660 & 0 & 11,100 \\
\hline $11 \mathrm{AF}$ & $\begin{array}{rr}41 & 31 \\
122 & 23\end{array}$ & 139 & 1,850 & 0 & 72,000 \\
\hline${ }_{-}^{11 B 0}$ & $\begin{array}{rr}39 & 20 \\
122 & 30\end{array}$ & 102 & 1,820 & 265 & 50,880 \\
\hline$-^{11 G \mathrm{~A}}$ & $\begin{array}{rr}32 & 53 \\
116 & 48\end{array}$ & 190 & 1,580 & 59 & 112,810 \\
\hline$-11 \mathrm{GE}$ & \begin{tabular}{rr|}
34 & 08 \\
118 & 31
\end{tabular} & 14 & 163 & 2.4 & 10,300 \\
\hline $\begin{array}{r}11 C J \\
11-417950\end{array}$ & $\begin{array}{rr}39 & 14 \\
121 & 16\end{array}$ & 1,110 & 815 & 25,000 & 70,000 \\
\hline $1 \mathrm{NE}$ & $\begin{array}{rr}34 & 42 \\
118 & 26\end{array}$ & - & 172 & 0 & 7,507 \\
\hline$\Omega^{10 B A}$ & $\begin{array}{rr}38 & 55 \\
120 & 04\end{array}$ & 157 & 1,408 & 0 & $a 6,800$ \\
\hline $10 \mathrm{BA}$ & $\begin{array}{rr}38 & 53 \\
120 & 04\end{array}$ & 157 & 1,410 & - & - \\
\hline $\begin{array}{r}11 \mathrm{FR} \\
11-304410\end{array}$ & $\begin{array}{rr}37 & 55 \\
120 & 56\end{array}$ & 212 & 4,100 & 0 & 52,000 \\
\hline $\begin{array}{r}11 F F \\
11-229600\end{array}$ & $\begin{array}{rr}37 & 16 \\
118 & 58\end{array}$ & 171 & 962 & 168 & 64,570 \\
\hline
\end{tabular}

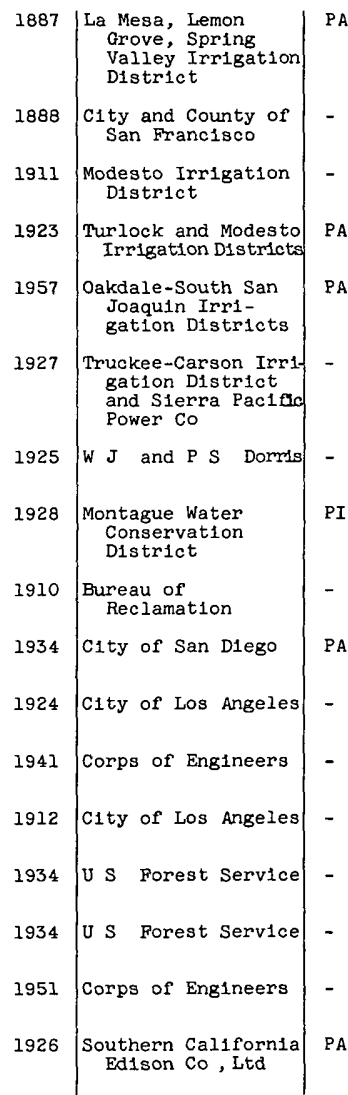

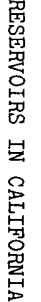




\begin{tabular}{|c|c|c|c|c|c|c|c|c|c|c|c|c|}
\hline \multirow[b]{2}{*}{$\begin{array}{l}\text { Name of reservolr } \\
\text { and } \\
\text { stream }\end{array}$} & \multicolumn{2}{|c|}{ Location } & \multirow{2}{*}{$\begin{array}{c}\text { Drainage } \\
\text { area } \\
\text { (square } \\
\text { m1les) }\end{array}$} & \multirow[b]{2}{*}{$\begin{array}{c}\text { Surface } \\
\text { area } \\
\text { (acres) }\end{array}$} & \multicolumn{3}{|c|}{ Storage in acre-feet } & \multirow[b]{2}{*}{$\begin{array}{c}\text { storage } \\
\text { rat10 }\end{array}$} & \multirow[b]{2}{*}{ Use } & \multirow[b]{2}{*}{$\begin{array}{c}\text { Date } \\
\text { com- } \\
\text { pleted }\end{array} \mid$} & \multirow[b]{2}{*}{$\begin{array}{l}\text { Owner } \\
\text { or } \\
\text { operator }\end{array}$} & \multirow{2}{*}{$\begin{array}{l}\text { Storage } \\
\text { records } \\
\text { pub- } \\
\text { 11shed }\end{array}$} \\
\hline & $\begin{array}{c}\text { Basin } \\
\text { 1ndex and } \\
\text { station no }\end{array}$ & $\begin{array}{l}\text { Latitude } \\
\text { and } \\
\text { long1tude }\end{array}$ & & & Dead & Total & Usable & & & & & \\
\hline $\begin{array}{l}\text { CALIFORNIA--Cont1nued } \\
\text { Folsom Lake } \\
\text { Amer1can River }\end{array}$ & $\mid \begin{array}{r}110 H \\
11-446200\end{array}$ & $\begin{array}{rr}38 & 42 \\
121 & 09\end{array}$ & 1,863 & 11,450 & 89,800 & - & $1,010,300$ & 04 & FIP & 1956 & $\begin{array}{l}\text { Bureau of } \\
\text { Reclamation }\end{array}$ & PA \\
\hline $\begin{array}{l}\text { French Lake } \\
\text { Canyon Creek }\end{array}$ & $\begin{array}{r}11 \mathrm{CK} \\
11-414400\end{array}$ & $\begin{array}{rr}39 & 25 \\
120 & 32\end{array}$ & 56 & 337 & 890 & 14,730 & 13,840 & - & IMP & 1859 & $\begin{array}{l}\text { Nevada Irrigation } \\
\text { District }\end{array}$ & - \\
\hline $\begin{array}{l}\text { Frenchman } \\
\text { L1ttle Last Chance Creek }\end{array}$ & $-11 \mathrm{CA}$ & $\begin{array}{rr}39 & 54 \\
120 & 12\end{array}$ & 82 & 1,470 & a3, 000 & - & 53,582 & 60 & IM & 1961 & $\begin{array}{l}\text { California State } \\
\text { Dept of Water Res }\end{array}$ & - \\
\hline 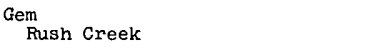 & $\begin{array}{r}10 E E \\
10-287280\end{array}$ & $\begin{array}{rr}37 & 45 \\
119 & 08\end{array}$ & 216 & 275 & - & - & 17,604 & 3 & IP & 1917 & $\begin{array}{c}\text { Cal1fornia Electric } \\
\text { Power Co }\end{array}$ & - \\
\hline $\begin{array}{l}\text { Gibraltar } \\
\text { Santa Ynez River }\end{array}$ & $-11 \mathrm{GH}$ & $\begin{array}{rr}34 & 31 \\
119 & 41\end{array}$ & 216 & 415 & 716 & 14,777 & 14,061 & 5 & M & 1920 & $\begin{array}{l}\text { C1ty of Santa } \\
\text { Barbara }\end{array}$ & PA \\
\hline $\begin{array}{l}\text { Grant Lake } \\
\text { Rush Creek }\end{array}$ & $-10 \mathrm{EE}$ & $\begin{array}{rr}37 & 50 \\
119 & 06\end{array}$ & 60 & 1,095 & 2,600 & 50,125 & 47,525 & 8 & M & 1940 & C1ty of Los Angeles & - \\
\hline $\begin{array}{l}\text { Haiwee } \\
\text { Los Angeles aqueduct }\end{array}$ & $\begin{array}{r}109 \mathrm{~A} \\
10-264860\end{array}$ & $\begin{array}{rr}36 & 10 \\
117 & 56\end{array}$ & 89 & 1,804 & 2,300 & 58,525 & 56,225 & - & M & 1913 & C1ty of Los Angeles & - \\
\hline $\begin{array}{l}\text { Hansen } \\
\text { Tu Junga Creek }\end{array}$ & $-11 \mathrm{GE}$ & $\begin{array}{rr}34 & 16 \\
118 & 22\end{array}$ & 147 & 1,090 & 1,370 & 33,370 & 32,000 & 17 & $F$ & 1940 & Corps of Engineers & - \\
\hline $\begin{array}{l}\text { Havasu Lake (Parker Dam)* } \\
\text { Colorado River }\end{array}$ & $09-42759 \mathrm{gLB}$ & $\begin{array}{rr}34 & 18 \\
114 & 08\end{array}$ & 178,800 & 20,390 & 0 & 619,400 & 180,000 & - & FIMPR & 1938 & $\begin{array}{l}\text { Bureau of } \\
\text { Reclamation }\end{array}$ & $\mathrm{PA}$ \\
\hline $\begin{array}{l}\text { Haynes } \\
\text { Goose Creek }\end{array}$ & $-11 \mathrm{BE}$ & $\begin{array}{rr}40 & 54 \\
121 & 45\end{array}$ & 53 & 242 & 0 & 6,600 & 6,600 & - & IM & 1963 & Welch and Welch & - \\
\hline $\begin{array}{l}\text { Hernandez } \\
\text { San Benito River }\end{array}$ & $-11 \mathrm{FH}$ & $\begin{array}{rr}36 & 24 \\
120 & 50\end{array}$ & 88 & 590 & 700 & 18,700 & 18,000 & 25 & IR & 1963 & $\begin{array}{l}\text { San Ben1 to County } \\
\text { Flood Control and } \\
\text { Water Conserv D1st }\end{array}$ & - \\
\hline $\begin{array}{l}\text { Hetch Hetchy (O'Shaughnessy Dam) } \\
\text { Tuolumne R1ver }\end{array}$ & $11-275500$ & $\begin{array}{rr}37 & 57 \\
119 & 47\end{array}$ & 460 & 1,960 & 0 & 360,400 & 360,400 & 5 & MP & 1923 & $\begin{array}{c}\text { City and County of } \\
\text { San Francisco }\end{array}$ & $\mathrm{PA}$ \\
\hline $\begin{array}{l}\text { Hog Flat } \\
\text { Susan River }\end{array}$ & $-10 \mathrm{AJ}$ & $\begin{array}{rr}40 & 26 \\
120 & 54\end{array}$ & 110 & 1,000 & 0 & 8,000 & 8,000 & - & I & 1891 & $\begin{array}{l}\text { Lassen Irrigation } \\
\text { Co }\end{array}$ & - \\
\hline $\begin{array}{l}\text { Huntington Lake } \\
\text { B1g Creek }\end{array}$ & $\begin{array}{r}11 F G \\
11-236000\end{array}$ & $\begin{array}{rr}37 & 14 \\
119 & 13\end{array}$ & 790 & 1,441 & 600 & 89,800 & 89,200 & - & PR & 1914 & $\begin{array}{l}\text { Southern California } \\
\text { Ed1son Co, Ltd }\end{array}$ & PA \\
\hline $\begin{array}{l}\text { Ice House } \\
\text { South Fork Silver Creek }\end{array}$ & $\begin{array}{r}11 \mathrm{DE} \\
11-441100\end{array}$ & $\begin{array}{rr}38 & 49 \\
120 & 22\end{array}$ & 272 & 678 & 160 & 45,960 & 45,800 & 9 & $P$ & 1959 & $\begin{array}{c}\text { Sacramento Municipar } \\
\text { Utility District }\end{array}$ & PA \\
\hline $\begin{array}{l}\text { Imperial } \\
\text { Colorado River }\end{array}$ & $-9 L B$ & $\begin{array}{rr}32 & 53 \\
114 & 28\end{array}$ & 187,000 & 7,300 & - & 85,000 & - & - & I & 1938 & $\begin{array}{l}\text { Bureau of } \\
\text { Reclamation }\end{array}$ & - \\
\hline
\end{tabular}


Independence Lake

Independence Creek

Iron Gate

Klamath R1ver

Isabella

Jameson Lake (Juncal Dam)

Santa Ynez River

Jenkinson Lake (Siy Park Dam) Sly Park Creek

Kent Lake

Lagunitas Creek

Kesw1ck

Sacramento River

Lafayette

Lafayette Creek

Lake Almanor

North Fork Feather R1ver

Lake Arrowhead (Little Bear)

wake Berryess

Lake Britton (P1t No 3)

Lake Cachuma
Santa Ynez River

Lake clementine

North Fork American River

Lake Crowley (Long Valley Dam)

Lake Curry

Lake Eleanor
Eleanor Creek

Lake Elsman (Austrian Dam)

Los Gatos Creek

Lake Fordyce

Fordyce Creek

See footnotes at end of table

\begin{tabular}{|c|c|}
\hline${ }_{-}^{10 B B}$ & $\begin{array}{rr}39 & 27 \\
120 \quad 17\end{array}$ \\
\hline$-^{11 \mathrm{AM}}$ & $\begin{array}{r}415 \\
1222\end{array}$ \\
\hline $\begin{array}{r}11 \mathrm{FC} \\
11-190500\end{array}$ & $\begin{array}{r}353 \\
1182\end{array}$ \\
\hline $\begin{array}{r}11 \mathrm{GH} \\
11-1210^{0} 05\end{array}$ & $\begin{array}{r}34 \\
119\end{array}$ \\
\hline$-^{11 D M}$ & $\begin{array}{r}384 \\
1203\end{array}$ \\
\hline$-^{11 E L}$ & $\begin{array}{r}375 \\
1224\end{array}$ \\
\hline $\begin{array}{r}11 \mathrm{BE} \\
11-370460\end{array}$ & $\begin{array}{r}40 \\
122\end{array}$ \\
\hline $\begin{array}{r}11 \mathrm{GP} \\
11-182910\end{array}$ & $\begin{array}{r}375 \\
1220\end{array}$ \\
\hline $\begin{array}{r}11 C B \\
11-399000\end{array}$ & $\begin{array}{rl}40 & 1 \\
121 & 0\end{array}$ \\
\hline$-10 \mathrm{NE}$ & $\begin{array}{r}341 \\
1171\end{array}$ \\
\hline $\begin{array}{r}11 D C \\
11-453900\end{array}$ & $\begin{array}{r}383 \\
1220\end{array}$ \\
\hline $\begin{array}{r}11 \mathrm{BE} \\
11-361400\end{array}$ & $\begin{array}{r}410 \\
1214\end{array}$ \\
\hline $\begin{array}{r}11 \mathrm{GH} \\
11-125500\end{array}$ & $\begin{array}{r}343 \\
1195\end{array}$ \\
\hline$-^{11 D D}$ & $\begin{array}{r}38 \\
121\end{array}$ \\
\hline$-100 \mathrm{~A}$ & $\begin{array}{r}373 \\
1184\end{array}$ \\
\hline$-^{2100}$ & $\begin{array}{r}38 \\
122\end{array}$ \\
\hline $\begin{array}{r}11 \text { FMM } \\
11-277500\end{array}$ & $\begin{array}{r}37 \\
119\end{array}$ \\
\hline $\begin{array}{r}11 G 0 \\
11-167950\end{array}$ & $\begin{array}{r}37 \\
121\end{array}$ \\
\hline $11 \mathrm{CK}$ & $\begin{array}{r}39 \\
120\end{array}$ \\
\hline
\end{tabular}

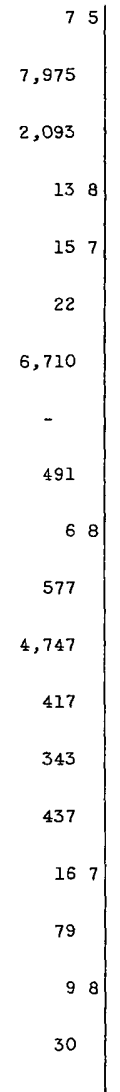

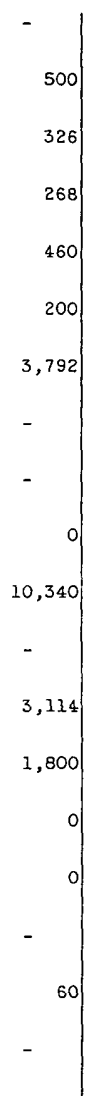

570,000

6,590

41,030

16,500

$-$

$-$

47,000

$1,602,300$

$-$

204,870

14,600

183,500

10,700

$-$

6,150

46,700 .

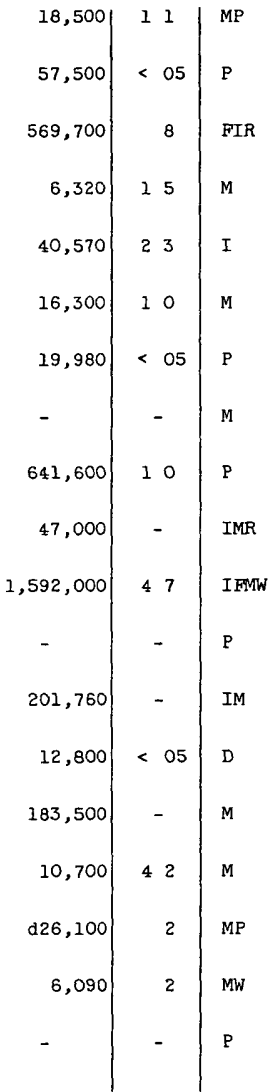

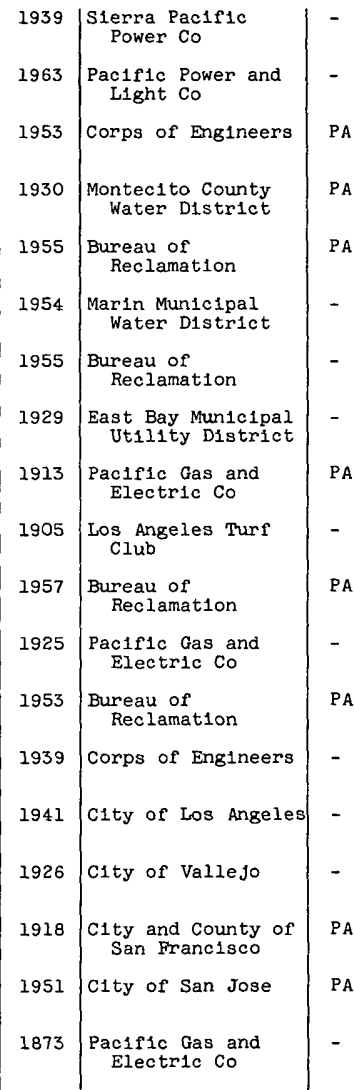

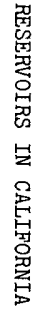


Table 1 --Reservo1rs in the Un1ted States completed or under construction as of January 1, 1963, hav1ng a usable capac1ty of 5,000 acre-feet or more--Continued

\begin{tabular}{|c|c|c|c|c|c|c|c|c|c|c|c|c|}
\hline \multirow[b]{2}{*}{$\begin{array}{l}\text { Name of reservoir } \\
\text { and } \\
\text { stream }\end{array}$} & \multicolumn{2}{|c|}{ Location } & \multirow{2}{*}{$\begin{array}{l}\text { Drainage } \\
\text { area } \\
\text { (square } \\
\text { m1les) }\end{array}$} & \multirow[b]{2}{*}{$\begin{array}{c}\text { Surface } \\
\text { area } \\
\text { (acres) }\end{array}$} & \multicolumn{3}{|c|}{ Storage in acre-feet } & \multirow[b]{2}{*}{$\mid \begin{array}{c}\text { Storage } \\
\text { rat1o }\end{array}$} & \multirow[b]{2}{*}{ Use } & \multirow[b]{2}{*}{$\begin{array}{c}\text { Date } \\
\text { com- } \\
\text { pleted }\end{array} \mid$} & \multirow[b]{2}{*}{$\begin{array}{l}\text { Owner } \\
\text { or } \\
\text { operator }\end{array}$} & \multirow{2}{*}{$\begin{array}{l}\text { Storage } \\
\text { records } \\
\text { pub- } \\
\text { lished }\end{array}$} \\
\hline & \begin{tabular}{|c|} 
Basin \\
1ndex and \\
station no
\end{tabular} & $\begin{array}{l}\text { Latitude } \\
\text { and } \\
\text { long1tude }\end{array}$ & & & Dead & Total & Usable & & & & & \\
\hline CALIFORNIA- Cont1nued & & & & & & & & & & & & \\
\hline $\begin{array}{l}\text { Lake Hemet } \\
\text { South Fork San Jacinto River }\end{array}$ & $11-069000$ & $\begin{array}{rr}33 & 40 \\
116 & 42\end{array}$ & 66 & 470 & 0 & 11,882 & 11,882 & 17 & IM & 1893 & $\begin{array}{l}\text { Lake Hemet Munic1- } \\
\text { pal Water District }\end{array}$ & $\mathrm{PA}$ \\
\hline $\begin{array}{l}\text { Lake Hennessey } \\
\text { Conn Creek }\end{array}$ & $-11 E L$ & $\begin{array}{rr}38 & 29 \\
122 & 22\end{array}$ & 523 & 850 & 0 & 31,000 & 31,000 & 12 & M & 1948 & C1ty of Napa & - \\
\hline $\begin{array}{l}\text { Lake Henshaw } \\
\text { San Luis Rey River }\end{array}$ & $-11 \mathrm{GA}$ & $\begin{array}{rr}33 & 14 \\
116 & 46\end{array}$ & 206 & 6,020 & 0 & 194,300 & 194,300 & 45 & IMR & 1923 & $\begin{array}{l}\text { V1sta Irrigation } \\
\text { District }\end{array}$ & PA \\
\hline $\begin{array}{l}\text { Lake Hodges } \\
\text { San Dieguito River }\end{array}$ & $-11 \mathrm{GA}$ & $\begin{array}{rr}33 & 03 \\
117 & 08\end{array}$ & 303 & 1,317 & 1,160 & 33,550 & 32,390 & 9 & M & 1918 & City of San Diego & $\mathrm{PA}$ \\
\hline $\begin{array}{l}\text { Lake Kaweah } \\
\text { Kaweah R1ver }\end{array}$ & $\begin{array}{r}11 F D \\
11-210900\end{array}$ & $\begin{array}{rl}36 & 25 \\
119 & 01\end{array}$ & 560 & 1,945 & 166 & 149,570 & 149,400 & 4 & FIR & 1961 & Corps of Engineers & $\mathrm{PA}$ \\
\hline $\begin{array}{l}\text { Lake Lev1tt } \\
\text { Susan River }\end{array}$ & ${ }_{-}^{10 \mathrm{AJ}}$ & $\begin{array}{rr}40 & 22 \\
120 & 30\end{array}$ & 93 & 2,560 & o) & 14,000 & 14,000 & - & I & 1891 & $\underset{\mathrm{Co}}{\text { Lassen Irrigation }}$ & - \\
\hline $\begin{array}{l}\text { Lake Mathews (Cajalco) } \\
\text { Cajalco Creek }\end{array}$ & $\quad \quad I I G D$ & $\begin{array}{rr}33 & 51 \\
117 & 26\end{array}$ & 40 & 2,750 & 3,546 & 182,000 & 278,500 & - & M & 1938 & $\begin{array}{l}\text { Metropolitan Water } \\
\text { D1st of So Callf }\end{array}$ & - \\
\hline $\begin{array}{l}\text { Lake McClure } \\
\text { Merced River }\end{array}$ & $11-269500$ & $\begin{array}{rr}37 & 35 \\
120 & 16\end{array}$ & 1,020 & 2,720 & 400 & 281,300 & 280,900 & 3 & IP & 1926 & $\begin{array}{l}\text { Merced Irrigation } \\
\text { District }\end{array}$ & PA \\
\hline $\begin{array}{l}\text { Lake Mendocino } \\
\text { East Fork Russian R1ver }\end{array}$ & $11-461800$ & $\begin{array}{rr}39 & 12 \\
123 & 11\end{array}$ & 205 & 1,960 & - & 122,500 & 118,000 & 5 & FIMRW & 1958 & Corps of Engineers & - \\
\hline $\begin{array}{l}\text { Lake Pillsbury } \\
\text { Eel River }\end{array}$ & $\begin{array}{r}11 E C \\
11-470000\end{array}$ & $\begin{array}{rr}39 & 24 \\
122 & 57\end{array}$ & 289 & 2,003 & 397 & 86,800 & 86,400 & 2 & IP & 1921 & $\begin{array}{l}\text { Pac1fic Gas and } \\
\text { Electric co }\end{array}$ & $\mathrm{PA}$ \\
\hline $\begin{array}{l}\text { Lake Piru } \\
\text { P1ru Creek }\end{array}$ & $\begin{array}{r}11 G F \\
11-109700\end{array}$ & $\begin{array}{rr}34 & 28 \\
118 & 45\end{array}$ & 424 & 1,240 & 74 & 101,225 & 101,150 & 32 & IM & 1955 & $\begin{array}{l}\text { United Water Con- } \\
\text { servation Dist }\end{array}$ & $\mathrm{PA}$ \\
\hline $\begin{array}{l}\text { Lake Spaulding } \\
\text { South Fork Yuba River }\end{array}$ & ${ }^{11 C K}$ & \begin{tabular}{rr|}
39 & 20 \\
120 & 38
\end{tabular} & 120 & 674 & - & 74,500 & - & - & $\mathrm{P}$ & 1912 & $\begin{array}{l}\text { Pac1fic Gas and } \\
\text { Electric Co }\end{array}$ & - \\
\hline $\begin{array}{l}\text { Lake Success } \\
\text { Tule River }\end{array}$ & $\begin{array}{r}11 \mathrm{FD} \\
11-204700\end{array}$ & $\begin{array}{rr}36 & 04 \\
118 & 55\end{array}$ & 391 & 2,450 & 0 & 83,900 & 83,900 & 9 & FIR & 1961 & Corps of Engineers & PA \\
\hline $\begin{array}{l}\text { Lake Tahoe } \\
\text { Truckee R1ver }\end{array}$ & $\begin{array}{r}10 \mathrm{BA} \\
10-337000\end{array}$ & $\begin{array}{rr}39 & 10 \\
120 & 08\end{array}$ & 506 & 120,000 & - & - & 744,600 & 41 & $I P R$ & 1874 & $\begin{array}{l}\text { Federal Court } \\
\text { Watermaster }\end{array}$ & PA \\
\hline $\begin{array}{l}\text { Lake Thomas A Edison } \\
\text { Mono Creek }\end{array}$ & $\begin{array}{r}11 \mathrm{FF} \\
11-231000\end{array}$ & $\begin{array}{rr}37 & 22 \\
118 & 59\end{array}$ & 889 & 1,890 & - & - & 125,000 & - & P & 1954 & $\begin{array}{c}\text { Southern Californ1a } \\
\text { Edison Co, Ltd }\end{array}$ & PA \\
\hline $\begin{array}{l}\text { Lake Valley } \\
\text { Amerlcan River }\end{array}$ & $-^{11 D D}$ & $\begin{array}{rr}39 & 18 \\
120 & 36\end{array}$ & 47 & 312 & - & - & - & - & $P$ & 1887 & $\begin{array}{l}\text { Pac1fic Gas and } \\
\text { Electric Co }\end{array}$ & - \\
\hline
\end{tabular}


Lake Van Norden

Lake Wilenor (Concow Dam)

Concow Creek

Lake Wohlford

Ficondido Creek

Lew1ston

Trinity River

Lexington

Los Gatos Creek

Little Grass Valley

South Fork Feather River

Loon Lake

Gerle Creek

Lost Creek
Lost Creek

Loveland

Sweetwater River

Lower Bear River

Lower Otay (Savage Dam)

Lower San Fernando

Lyons

South Fork Stanislaus RIver

Mammoth Pool

San Juaquin R1ver

Mariposa

Mariposa Creek

Matallja

lija Creek

McCoy Flat
Susan River

Meadow Lake Tributary to North Fork
Mokelumne Rdver

\section{Mediey Lakes}

See footnotes at end of table

\begin{tabular}{|c|c|}
\hline${ }_{-}^{11 \mathrm{CK}}$ & $\begin{array}{rr}39 & 19 \\
120 & 22\end{array}$ \\
\hline${ }_{-}^{11 C C}$ & $\begin{array}{rr}39 & 46 \\
121 & 31\end{array}$ \\
\hline $\begin{array}{r}11 \mathrm{GA} \\
11-030700\end{array}$ & $\begin{array}{rr}33 & 10 \\
117 & 00\end{array}$ \\
\hline $11 \mathrm{AM}$ & $\begin{array}{rr}40 & 43 \\
122 & 48\end{array}$ \\
\hline $11-1679 \frac{3190}{80}$ & $\begin{array}{rr}37 & 12 \\
121 & 59\end{array}$ \\
\hline $\begin{array}{r}11 C E \\
11-3950 \quad 20\end{array}$ & $\begin{array}{rr}39 & 43 \\
121 & 01\end{array}$ \\
\hline - $I I D E$ & $\begin{array}{rr}39 & 00 \\
120 & 18\end{array}$ \\
\hline $\begin{array}{r}11 C D \\
11-3956 \quad 00\end{array}$ & $\begin{array}{rr}39 & 34 \\
121 & 09\end{array}$ \\
\hline $11 \mathrm{GA}$ & $\begin{array}{ll}32 & 48 \\
3 & 6\end{array}$ \\
\hline $\begin{array}{r}11 \mathrm{DL} \\
11-315600\end{array}$ & $\begin{array}{rr}38 & 32 \\
120 & 15\end{array}$ \\
\hline $21 \mathrm{GA}$ & $\begin{array}{r}3237 \\
11656\end{array}$ \\
\hline $11 G E$ & $\begin{array}{rr}34 & 18 \\
118 & 28\end{array}$ \\
\hline $\begin{array}{r}11 \mathrm{FO} \\
11-297700\end{array}$ & $\begin{array}{rr}38 & 05 \\
120 & 11\end{array}$ \\
\hline $\begin{array}{r}11 \mathrm{FG} \\
11-2347\end{array}$ & $\begin{array}{r}3720 \\
11919\end{array}$ \\
\hline $\begin{array}{r}11 \mathrm{FJ} \\
11-260495\end{array}$ & $\begin{array}{rr}37 & 18 \\
120 & 09\end{array}$ \\
\hline $\begin{array}{r}11 \mathrm{GG} \\
11-1150\end{array}$ & $\begin{array}{r}34 \quad 29 \\
119 \quad 18\end{array}$ \\
\hline $10 \mathrm{AJ}$ & $\begin{array}{r}4027 \\
12057\end{array}$ \\
\hline $\begin{array}{r}11 D L \\
11-313483\end{array}$ & $\begin{array}{lll}38 & 36\end{array}$ \\
\hline $\mid \begin{array}{r}11 \mathrm{DG} \\
11-434900\end{array}$ & $\begin{array}{r}3852 \\
120 \quad 08\end{array}$ \\
\hline
\end{tabular}

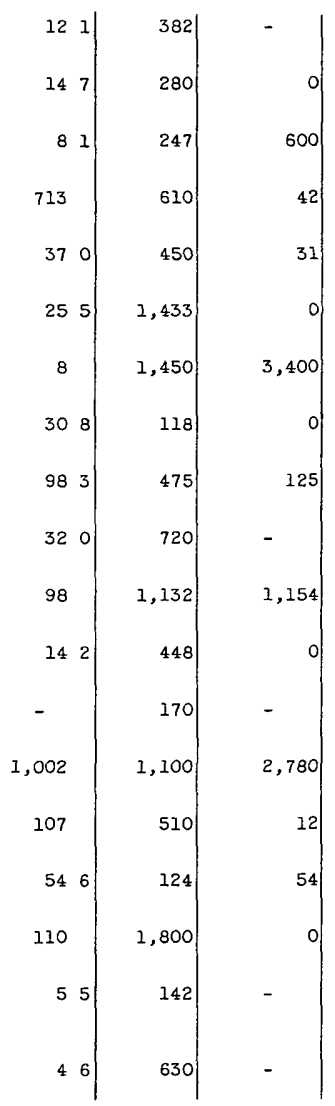

$d 5,260$
$d 8,210$
7,500
14,659
21,430
94,660
76,500
$d 5,105$
25,387
49,100
49,511
20,500
5,400
122,600
15,040
7,020
17,290
-\begin{tabular}{|}
5,000 \\
1
\end{tabular}

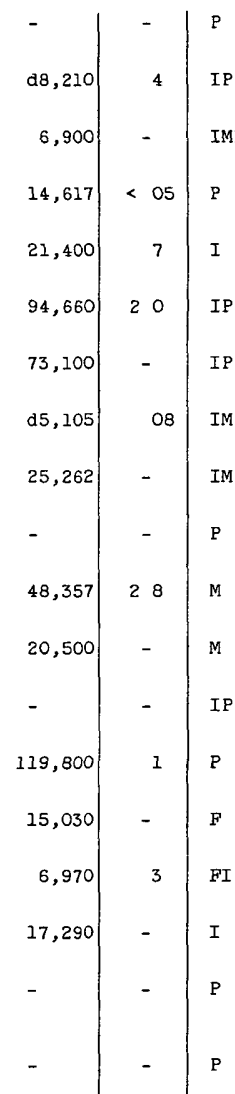

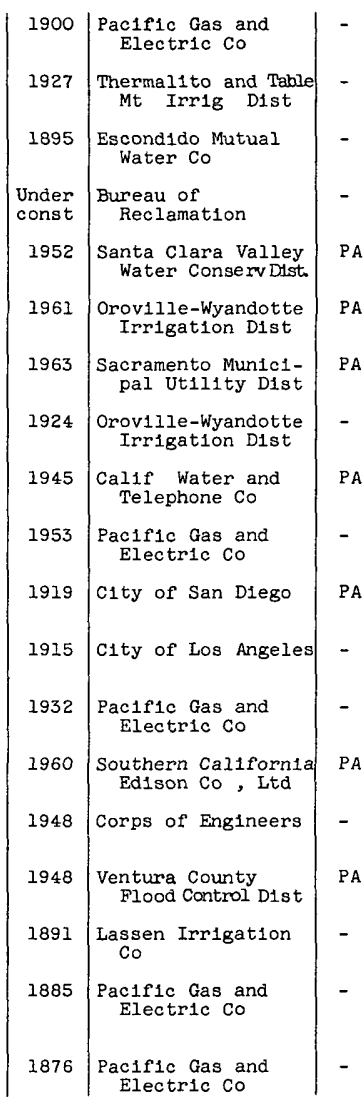



[For meaning of symbols under "Use" and "Storage records published," see text]

\begin{tabular}{|c|c|c|c|c|c|c|c|c|c|c|c|c|}
\hline \multirow[b]{2}{*}{$\begin{array}{l}\text { Name of reservolr } \\
\text { and } \\
\text { stream }\end{array}$} & \multicolumn{2}{|c|}{ Location } & \multirow{2}{*}{$\begin{array}{l}\text { Drainage } \\
\text { area } \\
\text { (square } \\
\text { miles) }\end{array}$} & \multirow[b]{2}{*}{$\begin{array}{c}\text { Surface } \\
\text { area } \\
\text { (acres) }\end{array}$} & \multicolumn{3}{|c|}{ Storage in acre-feet } & \multirow[b]{2}{*}{$\begin{array}{c}\text { Storage } \\
\text { rat10 }\end{array}$} & \multirow[b]{2}{*}{ Use } & \multirow[b]{2}{*}{$\begin{array}{c}\text { Date } \\
\text { com- } \\
\text { pleted }\end{array} \mid$} & \multirow[b]{2}{*}{$\begin{array}{l}\text { Owner } \\
\text { or } \\
\text { operator }\end{array}$} & \multirow{2}{*}{$\begin{array}{l}\text { Storage } \\
\text { records } \\
\text { pub- } \\
\text { l1shed }\end{array}$} \\
\hline & $\begin{array}{c}\text { Basin } \\
\text { 1ndex and } \\
\text { station no }\end{array}$ & $\begin{array}{l}\text { Lat1tude } \\
\text { and } \\
\text { longitude }\end{array}$ & & & Dead & Total & Usable & & & & & \\
\hline $\begin{array}{l}\text { Melones } \\
\text { Stan1slaus River }\end{array}$ & $\begin{array}{r}11 \mathrm{FP} \\
11-299000\end{array}$ & $\begin{array}{rr}37 & 57 \\
120 & 31\end{array}$ & 904 & 1,843 & 2,630 & 112,630 & 110,000 & 01 & IP & 1926 & $\begin{array}{c}\text { Oakdale and South } \\
\text { San Joaquin Irri- } \\
\text { gation Districts }\end{array}$ & PA \\
\hline $\begin{array}{l}\text { Millerton Lake } \\
\text { San Joaquin River }\end{array}$ & $11-250100$ & $\begin{array}{rr}37 & 00 \\
119 & 42\end{array}$ & 1,633 & 4,900 & 17,400 & 520,600 & 503,200 & 3 & $\mathrm{FI}$ & 1947 & $\begin{array}{l}\text { Bureau of } \\
\text { Reclamation }\end{array}$ & $\mathrm{PA}$ \\
\hline $\begin{array}{l}\text { Miramar } \\
\text { B1g Surr Creek }\end{array}$ & $-11 \mathrm{GA}$ & $\begin{array}{rr}32 & 55 \\
117 & 06\end{array}$ & 1 & 162 & 2,200 & 7,250 & 6,050 & - & M & 1960 & City of San Diego & - \\
\hline $\begin{array}{l}\text { Morena } \\
\text { Cottonwood Creek }\end{array}$ & $-11 \mathrm{GA}$ & $\begin{array}{rr}32 & 41 \\
116 & 33\end{array}$ & 120 & 1,475 & 654 & 50,210 & 49,560 & 58 & M & 1910 & C1ty of San Diego & PA \\
\hline $\begin{array}{l}\text { Morris } \\
\text { San Gabriel River }\end{array}$ & $-^{11 \mathrm{GE}}$ & \begin{tabular}{rr|}
34 & 11 \\
117 & 52
\end{tabular} & 210 & 420 & 0 & 35,000 & 35,000 & 3 & FM & 1935 & $\begin{array}{l}\text { Metropolitan Water } \\
\text { Dist of So Calis. }\end{array}$ & - \\
\hline $\begin{array}{l}\text { Mountain Meadows } \\
\text { Ham1lton Branch Feather R1ver }\end{array}$ & $11-398400$ & $\begin{array}{rr}40 & 17 \\
121 & 02\end{array}$ & 167 & - & - & 24,000 & - & - & $\mathrm{P}$ & 1924 & $\begin{array}{l}\text { Paciflc Gas and } \\
\text { Electric Co }\end{array}$ & - \\
\hline $\begin{array}{l}\text { Murray } \\
\text { Chapparel Canyon }\end{array}$ & $-11 \mathrm{GA}$ & $\begin{array}{rr}32 & 47 \\
117 & 03\end{array}$ & 38 & 200 & o & 6,085 & 6,085 & - & I & 1918 & City of San Diego & - \\
\hline $\begin{array}{l}\text { Nacimiento } \\
\text { Nacimiento RIver }\end{array}$ & $-^{11 \mathrm{GM}}$ & $\begin{array}{rr}35 & 46 \\
120 & 52\end{array}$ & 324 & 5,370 & 10,000 & 350,000 & 340,000 & 18 & FIMR & 1957 & $\begin{array}{l}\text { Monterey County } \\
\text { Flood Control and } \\
\text { Water Conserv Dist }\end{array}$ & - \\
\hline $\begin{array}{l}\text { New Camp Far West } \\
\text { Bear River }\end{array}$ & $-11 \mathrm{CL}$ & $\begin{array}{rr}39 & 03 \\
121 & 18\end{array}$ & 285 & 2,000 & 0 & 103,000 & 103,000 & 3 & $I$ & 1963 & $\begin{array}{l}\text { South Sutter water } \\
\text { District }\end{array}$ & - \\
\hline $\begin{array}{l}\text { Newell } \\
\text { Newell Creek }\end{array}$ & $-11 G P$ & $\begin{array}{rr}37 & 06 \\
122 & 04\end{array}$ & 83 & 172 & o & 8,400 & 8,400 & - & MR & 1961 & City of Santa Cruz & - \\
\hline $\begin{array}{l}\text { New Hogan } \\
\text { Calaveras RIver }\end{array}$ & ${ }_{-}^{11 D N}$ & $\begin{array}{rr}38 & 10 \\
120 & 47\end{array}$ & 363 & 4,410 & 157 & 325,010 & 324,850 & 21 & FIR & 1963 & Corps of Engineers & - \\
\hline $\begin{array}{l}\text { Nicas1o } \\
\text { N1cas1o Creek }\end{array}$ & $-11 E L$ & $\begin{array}{rr}38 & 05 \\
122 & 45\end{array}$ & 36 & 845 & 0 & 22,400 & 22,400 & 8 & M & 1961 & $\begin{array}{l}\text { Mar1n Municipal } \\
\text { Water District }\end{array}$ & - \\
\hline $\begin{array}{l}\text { N1mbus } \\
\text { American River }\end{array}$ & $-11 \mathrm{DA}$ & $\begin{array}{rr}38 & 38 \\
121 & 13\end{array}$ & 188 & 8,700 & 1,760 & 8,760 & 7,000 & $<05$ & IP & 1955 & $\begin{array}{l}\text { Bureau of } \\
\text { Reclamation }\end{array}$ & - \\
\hline $\begin{array}{l}\text { Oroville } \\
\text { Feather River }\end{array}$ & $-11 C F$ & $\begin{array}{rr}39 & 32 \\
121 & 29\end{array}$ & 3,611 & 15,500 & 700 & $3,499,300$ & $3,498,600$ & 8 & FIMP & $\begin{array}{l}\text { Under } \\
\text { const }\end{array}$ & State of Callfornia & $\mathrm{PA}$ \\
\hline $\begin{array}{l}\text { Pacheco Lake (North Fork) } \\
\text { North Fork Pacheco Creek }\end{array}$ & $11 \mathrm{GP}$ & $\begin{array}{rr}37 & 03 \\
121 & 18\end{array}$ & 686 & 197 & of & 6,150 & 6,150 & - & I & 1939 & \begin{tabular}{|} 
Pacheco Pass Water \\
District
\end{tabular} & - \\
\hline
\end{tabular}




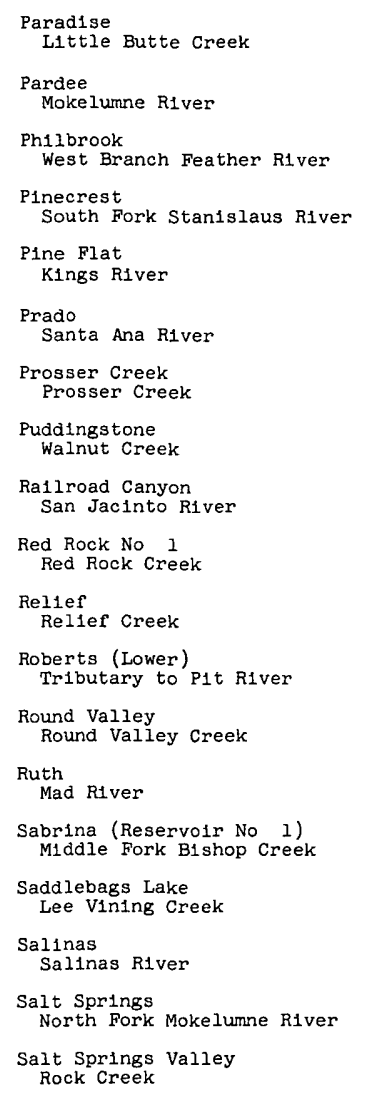

\begin{tabular}{|c|c|}
\hline$-^{11 C c}$ & $\begin{array}{rr}39 & 51 \\
121 & 34\end{array}$ \\
\hline $\begin{array}{r}11 \mathrm{DK} \\
11-320000\end{array}$ & $\begin{array}{rr}38 & 15 \\
120 & 51\end{array}$ \\
\hline$-^{11 \mathrm{CC}}$ & $\begin{array}{rr}40 & 02 \\
121 & 28\end{array}$ \\
\hline $\begin{array}{r}11 F 0 \\
11-295900\end{array}$ & $\begin{array}{rr}38 & 12 \\
119 & 59\end{array}$ \\
\hline $\begin{array}{r}11 \mathrm{FE} \\
11-221000\end{array}$ & $\begin{array}{rr}36 & 50 \\
119 & 19\end{array}$ \\
\hline$-^{11 G E}$ & $\begin{array}{r}3354 \\
117 \quad 38\end{array}$ \\
\hline$-^{10 B A}$ & $\begin{array}{rr}39 & 23 \\
120 & 08\end{array}$ \\
\hline $\begin{array}{r}11 \mathrm{GE} \\
11-085500\end{array}$ & $\begin{array}{rr}34 & 06 \\
117 & 48\end{array}$ \\
\hline $11 \mathrm{GD}$ & $\begin{array}{rr}33 & 42 \\
117 & 16\end{array}$ \\
\hline$-^{10 \mathrm{AK}}$ & $\begin{array}{rr}40 & 59 \\
120 & 09\end{array}$ \\
\hline $\begin{array}{r}11 \mathrm{FO} \\
11-291000\end{array}$ & $\begin{array}{rr}38 & 17 \\
119 & 44\end{array}$ \\
\hline$--^{11 \mathrm{BE}}$ & $\begin{array}{rr}41 & 13 \\
121 & 09\end{array}$ \\
\hline$-^{10 \mathrm{AJ}}$ & $\begin{array}{rr}40 & 31 \\
120 & 39\end{array}$ \\
\hline$-^{11 E B}$ & $\begin{array}{r}4022 \\
123 \\
25\end{array}$ \\
\hline$-^{10 G \mathrm{~A}}$ & $\begin{array}{rr}37 & 13 \\
118 & 37\end{array}$ \\
\hline$-^{10 E E}$ & $\begin{array}{rr}37 & 58 \\
119 & 16\end{array}$ \\
\hline $\begin{array}{r}11 \mathrm{GK} \\
11-1445 \text { OO }\end{array}$ & $\begin{array}{r}3520 \\
12030\end{array}$ \\
\hline $\begin{array}{r}11 \mathrm{FP} \\
11-313500\end{array}$ & $\begin{array}{rr}38 & 30 \\
120 & 13\end{array}$ \\
\hline $11 \mathrm{FR}$ & $\begin{array}{rr}38 & 02 \\
120 & 45\end{array}$ \\
\hline
\end{tabular}

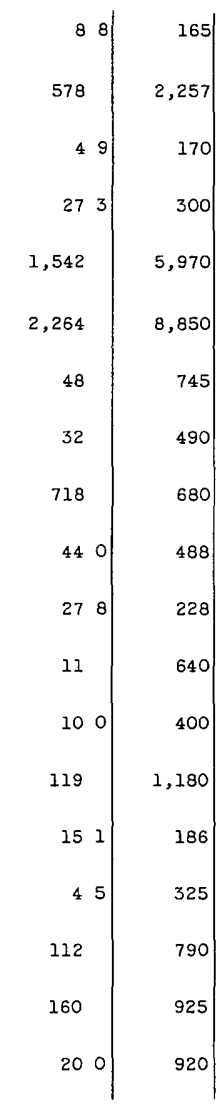


Table 1 --Reservoirs in the United States completed or under construction as of January 1, 1963, having a usable capac1ty of 5,000 acre-feet or more--Cont1nued

\begin{tabular}{|c|c|c|c|c|c|c|c|c|c|c|c|c|}
\hline \multirow[b]{2}{*}{$\begin{array}{l}\text { Name of reservo1r } \\
\text { and } \\
\text { stream }\end{array}$} & \multicolumn{2}{|c|}{ Location } & \multirow{2}{*}{$\begin{array}{l}\text { Drainage } \\
\text { area } \\
\text { (square } \\
\text { m1les) }\end{array}$} & \multirow[b]{2}{*}{$\begin{array}{c}\text { Surface } \\
\text { area } \\
\text { (acres) }\end{array}$} & \multicolumn{3}{|c|}{ Storage in acre-feet } & \multirow[b]{2}{*}{$\mid \begin{array}{c}\text { storage } \\
\text { rat1o }\end{array}$} & \multirow[b]{2}{*}{ Use } & \multirow[b]{2}{*}{$\begin{array}{c}\text { Date } \\
\text { com- } \\
\text { pleted }\end{array} \mid$} & \multirow[b]{2}{*}{$\begin{array}{l}\text { Owner } \\
\text { or } \\
\text { operator }\end{array}$} & \multirow{2}{*}{$\begin{array}{l}\text { Storage } \\
\text { records } \\
\text { pub- } \\
\text { lished }\end{array}$} \\
\hline & \begin{tabular}{|c|} 
Basin \\
1ndex and \\
station no
\end{tabular} & $\begin{array}{l}\text { Latitude } \\
\text { and } \\
\text { long1tude }\end{array}$ & & & Dead & Total & Usable & & & & & \\
\hline CALIFORNIA--Cont1n & & & & & & & & & & & & \\
\hline $\begin{array}{l}\text { San Andreas } \\
\text { San Andreas Creek }\end{array}$ & $\begin{array}{r}11 G P \\
11-1627 \quad 30\end{array}$ & $\begin{array}{rr}37 & 35 \\
122 & 25\end{array}$ & $\begin{array}{ll}4 & 4\end{array}$ & 550 & - & - & - & - & M & 1870 & $\begin{array}{l}\text { C1ty and County or } \\
\text { San Francisco }\end{array}$ & - \\
\hline $\begin{array}{l}\text { San Antonio } \\
\text { San Antonio Creek }\end{array}$ & $-11 G D$ & $\begin{array}{rr}34 & 10 \\
117 & 41\end{array}$ & 27 & 139 & o & 9,285 & 9,285 & 06 & F & 1956 & Corps of Eng1neers & - \\
\hline $\begin{array}{l}\text { San Gabriel No } 1 \\
\text { San Gabrlel River }\end{array}$ & $-^{11 G E}$ & $\begin{array}{rr}34 & 13 \\
117 & 51\end{array}$ & 203 & 560 & 2,000 & 44,600 & 42,600 & 4 & $\mathrm{CF}$ & 1938 & $\begin{array}{l}\text { Los Angeles County } \\
\text { Flood Control D1st }\end{array}$ & - \\
\hline $\begin{array}{l}\text { San Pablo } \\
\text { San Pablo Creek }\end{array}$ & $\begin{array}{r}11 G \mathrm{GP} \\
11-184090\end{array}$ & $\begin{array}{rr}37 & 56 \\
122 & 16\end{array}$ & 32 & 854 & - & - & - & - & M & 1920 & $\begin{array}{l}\text { East Bay Municipal } \\
\text { Ut111ty District }\end{array}$ & - \\
\hline $\begin{array}{l}\text { Santa Fe } \\
\text { San Gabriel RIver }\end{array}$ & $-^{11 \mathrm{GE}}$ & $\begin{array}{rr}34 & 07 \\
117 & 58\end{array}$ & 231 & 1,250 & 아 & 33,400 & 33,400 & - & F & 1949 & Corps of Engineers & - \\
\hline $\begin{array}{l}\text { Sant1ago } \\
\text { Sant1ago Creek }\end{array}$ & $-^{1 O G D}$ & $\begin{array}{rr}33 & 47 \\
117 & 43\end{array}$ & 632 & 650 & 0 & 25,000 & 25,000 & 21 & IF & 1933 & \begin{tabular}{|c|} 
Serrano and Carpen- \\
ter Irr1g DIsts \\
and Irv1ne County
\end{tabular} & $\mathrm{PA}$ \\
\hline $\begin{array}{l}\text { San Vicente } \\
\text { San V1cente Creek }\end{array}$ & $-11 \mathrm{GA}$ & $\begin{array}{rr}32 & 55 \\
116 & 55\end{array}$ & 75 & 1,069 & 350 & 90,230 & 89,880 & - & M & 1943 & City of San Diego & - \\
\hline $\begin{array}{l}\text { Schoeller Lake } \\
\text { Antelope Valley Basin }\end{array}$ & $-^{1 O N E}$ & $\begin{array}{rr}34 & 33 \\
118 & 07\end{array}$ & 7 & 288 & 0 & 6,575 & 6,575 & - & I & 1891 & $\begin{array}{l}\text { Palmdale Irri- } \\
\text { gation District }\end{array}$ & - \\
\hline $\begin{array}{l}\text { Scotts Flat } \\
\text { Deer Creek }\end{array}$ & $\begin{array}{r}11 C J \\
11-418250\end{array}$ & $\begin{array}{rr}39 & 17 \\
120 & 55\end{array}$ & 20 & 530 & 0 & 49,000 & 49,000 & 19 & IM & 1948 & $\begin{array}{l}\text { Nevada Irr1gation } \\
\text { Distr1ct }\end{array}$ & - \\
\hline $\begin{array}{l}\text { Sepulveda } \\
\text { Los Angeles RIver }\end{array}$ & $-^{11 G E}$ & $\begin{array}{rr}34 & 10 \\
118 & 28\end{array}$ & 155 & 1,900 & o & 17,300 & 27,300 & 11 & F & 1941 & Corps of Eng1neers & - \\
\hline $\begin{array}{l}\text { Shasta Lake } \\
\text { Sacramento River }\end{array}$ & $\begin{array}{r}11 \mathrm{BG} \\
11-3700 \quad 00\end{array}$ & $\begin{array}{rr}40 & 43 \\
122 & 25\end{array}$ & 6,665 & 29,500 & 115,700 & $4,492,700$ & $4,377,000$ & 7 & FIPR & 1949 & $\begin{array}{l}\text { Bureau of } \\
\text { Reclamation }\end{array}$ & $\mathrm{PA}$ \\
\hline $\begin{array}{l}\text { Shaver Lake } \\
\text { Stevenson Creek }\end{array}$ & $11-239500$ & $\begin{array}{rr}37 & 09 \\
119 & 18\end{array}$ & 291 & 2,177 & 92 & 135,400 & 135,300 & - & FIPR & 1927 & $\begin{array}{c}\text { Southern Cal1fornia } \\
\text { Edison Co }, \text { Ltd }\end{array}$ & $\mathrm{PA}$ \\
\hline $\begin{array}{l}\text { Silver Lake } \\
\text { Silver Fork }\end{array}$ & $\begin{array}{r}11 D G \\
11-4359\end{array}$ & $\begin{array}{rr}38 & 40 \\
120 & 08\end{array}$ & 142 & 510 & - & d 12,430 & - & - & $P$ & 1876 & $\begin{array}{l}\text { Pac1fic Gas and } \\
\text { Electric Co }\end{array}$ & $\mathrm{PA}$ \\
\hline $\begin{array}{l}\text { Sly Creek } \\
\text { Lost Creek }\end{array}$ & $\begin{array}{r}11 \mathrm{CD} \\
11-395400\end{array}$ & $\begin{array}{rr}39 & 35 \\
121 & 07\end{array}$ & 241 & 562 & 0 & 65,500 & 65,500 & - & IP & 1961 & $\begin{array}{l}\text { Orov1lle-Wyandotte } \\
\text { Irrigation D1st }\end{array}$ & $\mathrm{PA}$ \\
\hline $\begin{array}{l}\text { South Lake (H1isside Dam) } \\
\text { South Fork B1shop Creek }\end{array}$ & $-10 \mathrm{GA}$ & $\begin{array}{rr}37 & 10 \\
118 & 34\end{array}$ & 13 & 180 & - & - & 13,368 & 7 & IP & 1910 & $\mid \begin{array}{c}\text { California Electric } \\
\text { Power Co }\end{array}$ & - \\
\hline
\end{tabular}


Stone Canyon

Stony Gorge
Stony Creek

Stumpy Meadows

P1lot Creek

Sutherland

Santa Ysabel Creek

Sweetwater River

Tinemaha

Trinity Lake

Trinity RIve

Tule Lake
Cedar Creek

Tulloch

River

Turlock Lake (Owen Dam)

Tuolumne RIver

Twin Lakes

Cables Creek

Twitche11

ion valley

Silver Creek

Upper San Leandro

Uvas Creek

Vail Lake Temecula Creek

Villa Park

antiago Creek

Virgin1a Ranch Dry Creek

\begin{tabular}{|c|c|c|c|}
\hline$L^{1} \quad 1 G E$ & $\begin{array}{rr}34 & 06 \\
118 & 27\end{array}$ & $\begin{array}{ll}1 & 1\end{array}$ & 140 \\
\hline${ }_{-}^{11 B O}$ & $\begin{array}{rr}39 & 35 \\
122 & 32\end{array}$ & 301 & 1,275 \\
\hline$-11 D G$ & $\begin{array}{rr}38 & 54 \\
120 & 36\end{array}$ & 13 & 325 \\
\hline $11 \mathrm{GA}$ & $\begin{array}{rr}33 & 07\end{array}$ & $\begin{array}{ll}54 & 0\end{array}$ & 550 \\
\hline $11 G \mathrm{~A}$ & $\begin{array}{rr}32 & 41\end{array}$ & 180 & 1,030 \\
\hline $10 \mathrm{GA}$ & $\begin{array}{rr}117 & 00 \\
37 & 04\end{array}$ & 1,915 & 2,098 \\
\hline $11 \mathrm{BH}$ & $\begin{array}{rr}118 & 13 \\
40 & 48 \\
122 & 46\end{array}$ & 688 & 16,400 \\
\hline $\begin{array}{r}11 \mathrm{BC} \\
11-344800\end{array}$ & \begin{tabular}{rr|}
41 & 05 \\
120 & 25
\end{tabular} & 80 & 2,650 \\
\hline $\begin{array}{r}11 \mathrm{FP} \\
11-299995\end{array}$ & $\begin{array}{rr}37 & 52 \\
120 & 36\end{array}$ & 974 & 1,260 \\
\hline $11 \mathrm{FN}$ & & & \\
\hline $11-289600$ & $\begin{array}{lll}120 & 33\end{array}$ & & \\
\hline $\begin{array}{r}11 D G \\
11-436950\end{array}$ & $\begin{array}{rr}38 & 42 \\
120 & 04\end{array}$ & 127 & 620 \\
\hline $\begin{array}{r}11 G J \\
11-138050\end{array}$ & $\begin{array}{rr}34 & 59 \\
220 & 19\end{array}$ & 1,135 & 5,450 \\
\hline $\begin{array}{r}11 \mathrm{DE} \\
11-4410 \text { O1 }\end{array}$ & $\begin{array}{rr}38 & 52 \\
120 & 26\end{array}$ & 836 & 2,860 \\
\hline $\begin{array}{r}11 \mathrm{GP} \\
11-181150\end{array}$ & $\begin{array}{rr}37 & 46 \\
122 & 06\end{array}$ & $\begin{array}{ll}30 & 4\end{array}$ & 788 \\
\hline $\begin{array}{r}11 G 0 \\
11-154020\end{array}$ & $\begin{array}{rr}37 & 04 \\
121 & 41\end{array}$ & $\begin{array}{ll}30 & 2\end{array}$ & 280 \\
\hline $11 \mathrm{~GB}$ & 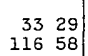 & 319 & 1,078 \\
\hline $11 \mathrm{GD}$ & 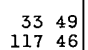 & 83 & 480 \\
\hline 110 & \begin{tabular}{rr|}
39 & 19 \\
121 & 19
\end{tabular} & 72 & 975 \\
\hline
\end{tabular}

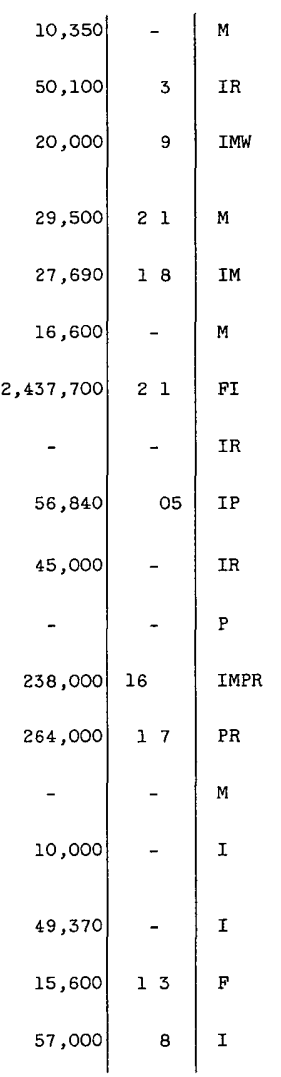


Table 1 --Reservoirs in the United States completed or under construction as of January 1, 1963, having a usable capac1ty of 5,000 acre-feet or more--Continued

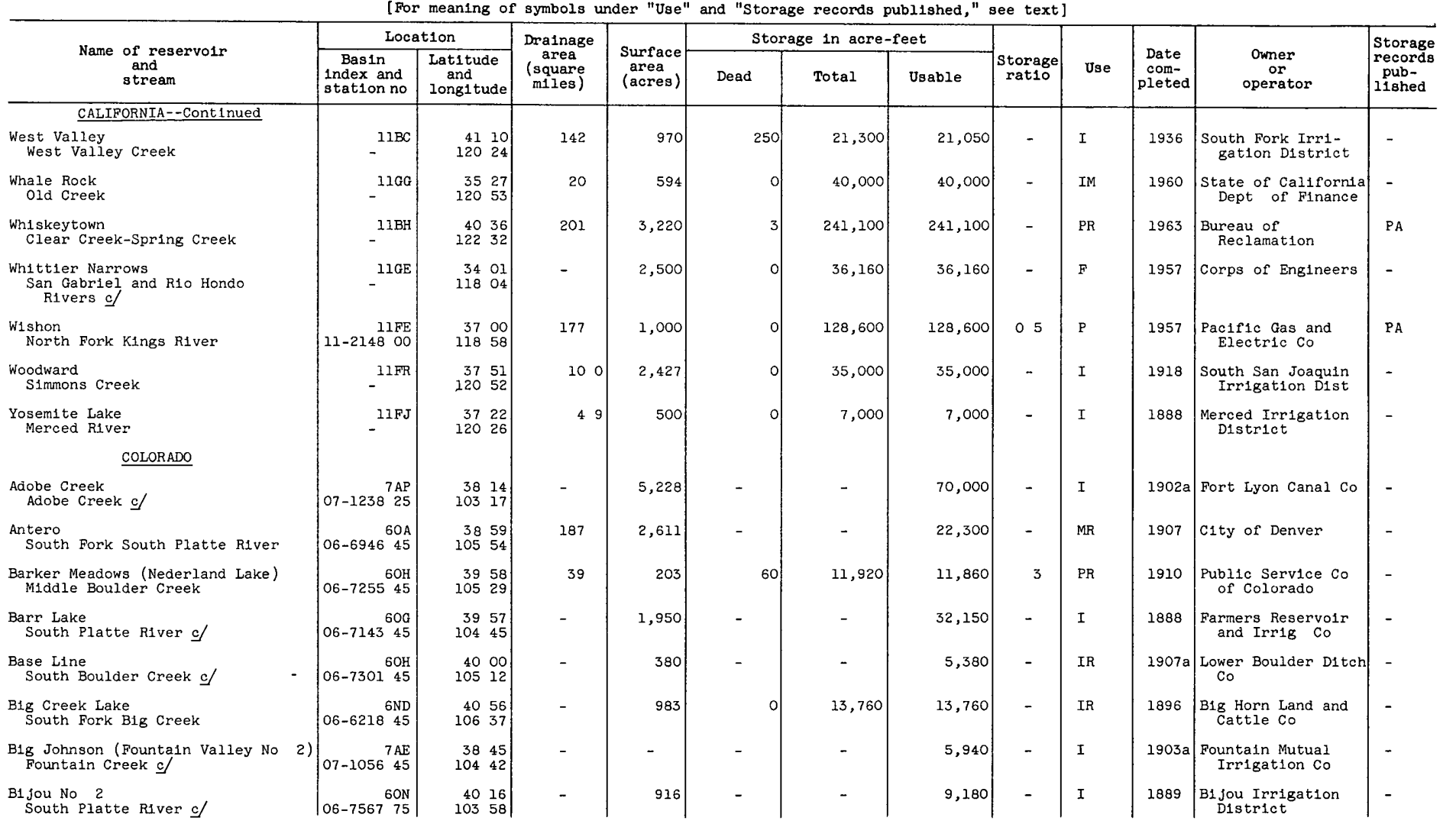




\begin{tabular}{|c|c|c|c|c|c|c|}
\hline $\begin{array}{l}\text { Black Hollow } \\
\text { Cache la Poudre River of }\end{array}$ & $06-7520^{60 L}$ & $\begin{array}{rr}40 & 37 \\
104 & 52\end{array}$ & - & - & - & - \\
\hline $\begin{array}{l}\text { Blue Lake No } 1 \\
\text { Bridal Ve1l Creek tributary }\end{array}$ & $09-1711 \quad 45$ & $\begin{array}{rr}37 & 53 \\
107 & 46\end{array}$ & - & - & _ & - \\
\hline $\begin{array}{l}\text { Bonny } \\
\text { South Fork Republican River }\end{array}$ & $\begin{array}{rr}6 \mathrm{RK} \\
06-8260 & 00\end{array}$ & $\begin{array}{rr}39 & 38 \\
102 & 11\end{array}$ & - & 5,036 & 1,420 & 170,100 \\
\hline $\begin{array}{l}\text { Boulder } \\
\text { Dry Creek c/ }\end{array}$ & $06-7253 \begin{array}{r}60 J \\
05\end{array}$ & $\begin{array}{rr}40 & 05 \\
105 & 13\end{array}$ & - & - & 0 & 13,500 \\
\hline $\begin{array}{l}\text { Boyd Lake } \\
\text { B1g Thompson River } c \text { / }\end{array}$ & $06-7413506$ & $\begin{array}{rr}40 & 25 \\
105 & 02\end{array}$ & - & 2,022 & 28,780 & 60,000 \\
\hline $\begin{array}{l}\text { Calkins Lake (Union) } \\
\text { St Vrain Creek cf }\end{array}$ & $06-7242 \quad 45$ & $\begin{array}{rr}40 & 10 \\
105 & 03\end{array}$ & - & 950 & - & - \\
\hline $\begin{array}{l}\text { Carter Lake } \\
\text { Water from Colorado River basin } \mathrm{c} /\end{array}$ & $06-7425 \quad 00 \mathrm{G}$ & $\begin{array}{rr}40 & 20 \\
105 & 13\end{array}$ & - & 1,161 & 3,310 & 116,800 \\
\hline $\begin{array}{l}\text { Chambers Lake } \\
\text { Joe Wright and Trap Creeks, } \\
\text { Fall R1ver } c /\end{array}$ & $06-7465 \quad 45$ & $\begin{array}{rr}40 & 36 \\
105 & 51\end{array}$ & - & 281 & - & - \\
\hline $\begin{array}{l}\text { Cheesman Lake } \\
\text { South Platte River }\end{array}$ & $06-7010^{600}$ & $\begin{array}{rr}39 & 12 \\
105 & 16\end{array}$ & 1,752 & 871 & 0 & 79,060 \\
\hline $\begin{array}{l}\text { Cherry Creek } \\
\text { Cherry Creek }\end{array}$ & $\begin{array}{r}60 \mathrm{E} \\
06-712990\end{array}$ & $\begin{array}{rr}39 & 39 \\
104 & 51\end{array}$ & 385 & 2,634 & o & 95,960 \\
\hline $\begin{array}{l}\text { Clear Creek } \\
\text { Clear Creek }\end{array}$ & $07-0869$ 7AA & $\begin{array}{rr}39 & 01 \\
106 & 15\end{array}$ & 62 & 414 & - & - \\
\hline $\begin{array}{l}\text { Cobb Lake } \\
\text { Cache la Poudre RIver } \mathrm{c}\end{array}$ & $06-751970$ & $\begin{array}{rr}40 & 39 \\
104 & 58\end{array}$ & - & - & - & - \\
\hline $\begin{array}{l}\text { Continental } \\
\text { North Clear Creek }\end{array}$ & $\begin{array}{r}8 B A \\
08-2144 \quad 00\end{array}$ & $\begin{array}{rr}37 & 53 \\
107 & 12\end{array}$ & a 50 & 773 & o & 26,720 \\
\hline $\begin{array}{l}\text { Cove Lake } \\
\text { San Antonio River c/ }\end{array}$ & $08-2482 \quad 45$ & $\begin{array}{rr}37 & 02 \\
105 & 53\end{array}$ & - & - & - & - \\
\hline $\begin{array}{l}\text { Cucharas Valley } \\
\text { Cucharas RIver }\end{array}$ & $07-1152 \begin{array}{r}7 \mathrm{AH} \\
45\end{array}$ & $\begin{array}{rr}37 & 45 \\
104 & 36\end{array}$ & - & - & - & - \\
\hline $\begin{array}{l}\text { Douglass Lake } \\
\text { Cache la Poudre River } \subseteq\end{array}$ & $06-7522 \quad 45$ & $\begin{array}{rr}40 & 42 \\
105 & 05\end{array}$ & - & - & - & - \\
\hline $\begin{array}{l}\text { Electra Lake } \\
\text { L1ttle Cascade and Elbert Creeks }\end{array}$ & $\begin{array}{rl}09-3597 & 45\end{array}$ & $\begin{array}{rr}37 & 33 \\
107 & 48\end{array}$ & - & 839 & 0 & 23,980 \\
\hline $\begin{array}{l}\text { Elevenmile Canyon } \\
\text { South Platte River }\end{array}$ & $\begin{array}{r}60 \mathrm{~A} \\
06-6955 \quad 00\end{array}$ & $\begin{array}{rr}38 & 54 \\
105 & 28\end{array}$ & 963 & 3,405 & 0 & 97,780 \\
\hline
\end{tabular}

\begin{tabular}{|c|c|c|c|c|}
\hline 7,480 & - & I & 1906 & $\begin{array}{c}\text { Water Supply and } \\
\text { Storage Co }\end{array}$ \\
\hline 6,014 & - & P & 1911 & $\begin{array}{l}\text { Smuggler Un1on } \\
\text { M1n1ng Co }\end{array}$ \\
\hline 168,700 & - & FIR & 2951 & $\begin{array}{l}\text { Bureau of } \\
\text { Reclamation }\end{array}$ \\
\hline 13,500 & - & FIR & 1955 & $\begin{array}{l}\text { Northern Colorado } \\
\text { Water Conservancy } \\
\text { District }\end{array}$ \\
\hline 31,200 & - & IR & 1902 & $\begin{array}{l}\text { Boyd Lake Reservolr } \\
\text { and Irr1g Co }\end{array}$ \\
\hline 12,740 & - & IR & 1902 & Union Reservoir co \\
\hline 113,500 & - & IPR & 1952 & $\begin{array}{l}\text { Bureau of } \\
\text { Reclamation }\end{array}$ \\
\hline 8,820 & - & IR & 1882 & $\begin{array}{l}\text { Water Supply and } \\
\text { Storage Co }\end{array}$ \\
\hline 79,060 & 7 & M & $1900 \mathrm{a}$ & C1ty of Denver \\
\hline 95,960 & - & $\mathrm{FR}$ & 1950 & Corps of Engineers \\
\hline 11,440 & 2 & IMR & $1902 \mathrm{a}$ & Pueblo Water Works \\
\hline 22,300 & - & I & 1919 & $\begin{array}{l}\text { Windsor Reservolr } \\
\text { and Canal Co }\end{array}$ \\
\hline 26,720 & 12 & IR & 1901 & $\begin{array}{l}\text { Santa Marla Reser- } \\
\text { vo1r Co }\end{array}$ \\
\hline 6,840 & - & I & 1893 & Pete M1ddlem1st \\
\hline 24,800 & - & $I$ & $1906 a$ & $\begin{array}{l}\text { Huerfano Valley } \\
\text { DItch Co }\end{array}$ \\
\hline 8,830 & - & I & 1901 & $\begin{array}{l}\text { Windsor Reservoir } \\
\text { and Canal Co }\end{array}$ \\
\hline 23,980 & - & P & 1902 & $\begin{array}{l}\text { West Colorado } \\
\text { Power Co }\end{array}$ \\
\hline 97,780 & 19 & MR & 1932 & C1ty of Denver \\
\hline
\end{tabular}




\begin{tabular}{|c|c|c|c|c|c|c|c|c|c|c|c|c|}
\hline \multirow[b]{2}{*}{$\begin{array}{l}\text { Name of reservo1r } \\
\text { and } \\
\text { stream }\end{array}$} & \multicolumn{2}{|c|}{ Location } & \multirow{2}{*}{$\begin{array}{c}\text { Drainage } \\
\text { area } \\
\text { (square } \\
\text { m1les) }\end{array}$} & \multirow[b]{2}{*}{$\begin{array}{c}\text { Surface } \\
\text { area } \\
\text { (acres) }\end{array}$} & \multicolumn{3}{|c|}{ Storage in acre-feet } & \multirow[b]{2}{*}{$\begin{array}{c}\text { Storage } \\
\text { rat10 }\end{array}$} & \multirow[b]{2}{*}{ Use } & \multirow[b]{2}{*}{$\begin{array}{c}\text { Date } \\
\text { com- } \\
\text { pleted }\end{array}$} & \multirow[b]{2}{*}{$\begin{array}{l}\text { Owner } \\
\text { or } \\
\text { operator }\end{array}$} & \multirow{2}{*}{$\begin{array}{l}\text { Storage } \\
\text { records } \\
\text { pub- } \\
\text { lished }\end{array}$} \\
\hline & $\begin{array}{l}\text { Basin } \\
\text { 1ndex and } \\
\text { station no }\end{array}$ & $\begin{array}{c}\text { Lat1 tude } \\
\text { and } \\
\text { long1tude }\end{array}$ & & & Dead & Total & Usable & & & & & \\
\hline COLORADO--Continued & & & & & & & & & & & & \\
\hline $\begin{array}{l}\text { Empire } \\
\text { South Platte River } c /\end{array}$ & $06-7565 \quad 55$ & $\begin{array}{rr}40 & 16 \\
104 & 11\end{array}$ & - & 2,842 & - & - & 37,710 & - & IR & 1906 & $\begin{array}{l}\text { B1 jou Irrigation } \\
\text { District }\end{array}$ & - \\
\hline $\begin{array}{l}\text { Fossil Creek } \\
\text { Cache la Poudre River } \mathrm{c}\end{array}$ & $\begin{array}{r}60 L \\
06-7522 \\
75\end{array}$ & $\begin{array}{rr}40 & 30 \\
105 & 00\end{array}$ & - & 840 & - & - & 11,510 & - & I & 1901 & $\begin{array}{l}\text { North Poudre Irri- } \\
\text { gation Co }\end{array}$ & - \\
\hline $\begin{array}{l}\text { Gould (Onion Valley) } \\
\text { Crystal Creek } c /\end{array}$ & $09-1292 \quad 45$ & $\begin{array}{rr}38 & 36 \\
107 & 35\end{array}$ & - & 560 & 0 & 9,170 & 9,170 & - & I & $1907 a$ & $\begin{array}{l}\text { Fruitland Irr1- } \\
\text { gation Co }\end{array}$ & - \\
\hline $\begin{array}{l}\text { Green Mountain } \\
\text { Blue River }\end{array}$ & 09-0570 90 & $\begin{array}{rr}39 & 53 \\
106 & 20\end{array}$ & 599 & 2,125 & 7,760 & 154,600 & 146,900 & 04 & IPR & 1942 & $\begin{array}{l}\text { Bureau of } \\
\text { Reclamation }\end{array}$ & PA \\
\hline $\begin{array}{l}\text { Gross } \\
\text { South Boulder Creek c/ }\end{array}$ & $06-729445$ & $\begin{array}{rr}39 & 57 \\
105 & 21\end{array}$ & - & 420 & 0 & 43,060 & 43,060 & - & MR & 1955 & $\begin{array}{l}\text { C1ty and County } \\
\text { of Denver }\end{array}$ & $\mathrm{PA}$ \\
\hline $\begin{array}{l}\text { Groundhog } \\
\text { Groundhog and L1ttle Fish Creeks }\end{array}$ & 09-1657 $\begin{array}{r}9 D L \\
55\end{array}$ & $\begin{array}{rr}37 & 47 \\
108 & 17\end{array}$ & also & 670 & 300 & 22,010 & 21,710 & - & I & $1905 \mathrm{a}$ & $\begin{array}{l}\text { Montezuma Valley } \\
\text { Irrigation Co }\end{array}$ & - \\
\hline $\begin{array}{l}\text { Gurley } \\
\text { Beaver Creek } \mathrm{c} /\end{array}$ & $09-1752 \quad 45$ & $\begin{array}{rr}38 & 02 \\
108 & 15\end{array}$ & - & - & - & 8,800 & - & - & IR & 1948 & $\begin{array}{l}\text { Farmers Water } \\
\text { Development co }\end{array}$ & - \\
\hline $\begin{array}{l}\text { Halligan } \\
\text { North Fork Cache la Poudre River }\end{array}$ & $\begin{array}{r}60 K \\
06-7511 \\
45\end{array}$ & $\begin{array}{rr}40 & 53 \\
105 & 20\end{array}$ & - & 253 & - & - & 6,430 & - & IR & 1910 & $\begin{array}{l}\text { North Poudre Irr1- } \\
\text { gation Co }\end{array}$ & - \\
\hline $\begin{array}{l}\text { Harvey Gap } \\
\text { East R1fle Creek } c /\end{array}$ & $09-0908 \quad 45$ & $\begin{array}{rr}39 & 37 \\
107 & 40\end{array}$ & - & - & - & 5,000 & - & - & IR & $1910 a$ & $\underset{\mathrm{CO}}{\text { Farmers Irrigation }}$ & - \\
\hline $\begin{array}{l}\text { Holbrook } \\
\text { Arkansas River } c \text { / }\end{array}$ & $07-1202 \quad 45$ & $\begin{array}{rr}38 & 04 \\
103 & 36\end{array}$ & - & 673 & - & - & 5,600 & - & IR & - & $\begin{array}{l}\text { Holbrook Mutual } \\
\text { Irrigation Co }\end{array}$ & - \\
\hline $\begin{array}{l}\text { Home Supply (Lonetree) } \\
\text { B1g Thompson River c/ }\end{array}$ & $\begin{array}{r}609 \\
06-7391 \quad 05\end{array}$ & $\begin{array}{rr}40 & 21 \\
105 & 07\end{array}$ & - & 536 & - & - & 9,270 & - & IR & 1882 & $\begin{array}{l}\text { Con-Home Supply } \\
\text { Reservolr Co }\end{array}$ & - \\
\hline $\begin{array}{l}\text { Horse Creek } \\
\text { South Platte River and Horse } \\
\text { Creek } c /\end{array}$ & $\mid \begin{array}{rr}60 \mathrm{M} \\
06-7567 & 25\end{array}$ & $\begin{array}{rl}40 & 01 \\
104 & 35\end{array}$ & - & - & - & - & 15,000 & - & I & 1911 & $\begin{array}{l}\text { Henrylen Irrigation } \\
\text { District }\end{array}$ & - \\
\hline $\begin{array}{l}\text { Horse Creek (Timber Lake) } \\
\text { Arkansas River and Horse Creek } c /\end{array}$ & $07-1236 \quad 25$ & $\begin{array}{rr}38 & 09 \\
103 & 24\end{array}$ & - & 2,456 & 4,010 & 29,010 & 25,000 & - & I & $1900 \mathrm{a}$ & Fort Lyon Canal Co & - \\
\hline $\begin{array}{l}\text { Horseshoe (Seven Lakes) } \\
\text { B1g Thompson River c) }\end{array}$ & $06-7413506$ & $\begin{array}{rr}40 & 26 \\
105 & 03\end{array}$ & - & 717 & - & - & 8,310 & - & IR & 1899 & $\begin{array}{l}\text { Seven Lakes Reser- } \\
\text { voir and Irrlg Co }\end{array}$ & - \\
\hline $\begin{array}{l}\text { Horsetooth } \\
\text { From Colorado River basin c/ }\end{array}$ & 06-7375 00 & $\begin{array}{rr}40 & 36 \\
105 & 10\end{array}$ & - & 1,899 & 8,270 & 151,800 & 143,500 & - & IR & 1949 & $\begin{array}{l}\text { Bureau of } \\
\text { Reclamation }\end{array}$ & PA \\
\hline
\end{tabular}




\begin{tabular}{|c|c|c|c|c|c|c|c|c|c|c|c|c|c|}
\hline $\begin{array}{l}\text { Ish } \\
\text { Little Thompson River } c /\end{array}$ & $\mid \begin{array}{r}60 G \\
06-742015\end{array}$ & $\begin{array}{rr}40 & 16 \\
105 & 06\end{array}$ & - & 384 & - & - & 7,060 & - & I & 1875 & $\begin{array}{l}\text { Boulder-Earimer } \\
\text { and Ish Counties }\end{array}$ & - & \\
\hline $\begin{array}{l}\text { Jackson Gulch } \\
\text { West Mancos R1ver }\end{array}$ & $\mid \begin{array}{r}9 \mathrm{GG} \\
09-3706^{4}\end{array}$ & $\begin{array}{rr}37 & 24 \\
108 & 15\end{array}$ & - & 218 & 29 & 10,160 & 10,130 & - & I & 1949 & $\begin{array}{l}\text { Mancos Conservation } \\
\text { District }\end{array}$ & - & \\
\hline $\begin{array}{l}\text { Jackson Lake } \\
\text { South Platte River } c \text { / }\end{array}$ & $\begin{array}{r}60 M \\
06-7569 \quad 25\end{array}$ & $\begin{array}{rr}40 & 22 \\
104 & 05\end{array}$ & - & 2,546 & - & - & 35,630 & - & IR & 1903 & $\begin{array}{c}\text { Jackson Lake Reser- } \\
\text { voir and Irrig Co }\end{array}$ & - & \\
\hline $\begin{array}{l}\text { John Martin (Formerly Caddoa) } \\
\text { Arkansas River }\end{array}$ & $07-1300 \begin{array}{r}7 \mathrm{AP} \\
00\end{array}$ & $\begin{array}{rr}38 & 04 \\
102 & 56\end{array}$ & 18,915 & 17,875 & 0 & 645,500 & 645,500 & 25 & FIR & - & Corps of Engineers & PA & \\
\hline $\begin{array}{l}\text { Julesburg (Jumbo) } \\
\text { South Platte R1ver c/ }\end{array}$ & $\begin{array}{r}60 P \\
06-760425\end{array}$ & $\begin{array}{rr}40 & 56 \\
102 & 38\end{array}$ & - & 1,560 & - & - & 27,220 & - & IR & 1905 & $\begin{array}{l}\text { Julesburg Irri- } \\
\text { gation District }\end{array}$ & - & \\
\hline $\begin{array}{l}\text { La Jara } \\
\text { La Jara Creek }\end{array}$ & $08-2373^{8 B C}$ & $\begin{array}{rr}37 & 15 \\
106 & 20\end{array}$ & - & 1,241 & 0 & 14,050 & 14,050 & 12 & IR & $1904 \mathrm{a}$ & $\underset{\text { Co }}{\text { Terrace Irrigation }}$ & - & \\
\hline $\begin{array}{l}\text { Lake Granby } \\
\text { Colorado River }\end{array}$ & 9DA & $\begin{array}{rr}40 & 09 \\
105 & 52\end{array}$ & 311 & 7,256 & 74,190 & 539,800 & 465,600 & - & IPR & 3949 & $\begin{array}{l}\text { Bureau of } \\
\text { Reclamation }\end{array}$ & PA & \\
\hline $\begin{array}{l}\text { Lake Henry } \\
\text { Arkansas River } c /\end{array}$ & $07-110655$ & $\begin{array}{rr}38 & 16 \\
103 & 42\end{array}$ & - & - & - & - & 6,300 & - & IW & 1891 & $\begin{array}{l}\text { National Sugar Mfg } \\
\text { Co }\end{array}$ & - & $\begin{array}{l}\text { 蛹 } \\
\text { 国 }\end{array}$ \\
\hline $\begin{array}{l}\text { Lake Loveland } \\
\text { B1g Thomps on River } \mathrm{c}\end{array}$ & $06-7413^{60 G}$ & $\begin{array}{rr}40 & 25 \\
105 & 05\end{array}$ & - & 472 & - & - & 14,240 & - & IR & $1893 \mathrm{a}$ & $\begin{array}{l}\text { Loveland Reservo1r } \\
\text { Irrigation Co }\end{array}$ & - & 苂 \\
\hline $\begin{array}{l}\text { Lake Meredith } \\
\text { Arkansas River } \mathrm{c}\end{array}$ & $\begin{array}{r}7 \mathrm{AK} \\
07-1106 \quad 65\end{array}$ & $\begin{array}{rr}38 & 09 \\
103 & 44\end{array}$ & 0 & 3,250 & - & - & 26,000 & - & IR & 1898 & $\begin{array}{l}\text { Twin Lakes Reser- } \\
\text { volr and Canal co. }\end{array}$ & - & 点 \\
\hline $\begin{array}{l}\text { Lake San Cristobal } \\
\text { Lake Fork }\end{array}$ & $09-1234 \quad 45$ & $\begin{array}{rr}37 & 59 \\
107 & 18\end{array}$ & - & 330 & - & - & 9,780 & - & MP & $1906 \mathrm{a}$ & $\begin{array}{l}\text { Bureau of } \\
\text { Reclamation }\end{array}$ & - & $\stackrel{8}{\circ}$ \\
\hline $\begin{array}{l}\text { Lower Latham } \\
\text { South Platte River } c /\end{array}$ & $06-7312^{60 G}$ & \begin{tabular}{rr|}
40 & 21 \\
104 & 38
\end{tabular} & - & 600 & - & - & 5,750 & - & IR & 1900 & $\begin{array}{l}\text { Lower Latham Reser- } \\
\text { voir Co }\end{array}$ & - & 兽 \\
\hline $\begin{array}{l}\text { MacFarlane } \\
\text { Illinols, Willow, and Soap } \\
\text { Creeks } c \text { / }\end{array}$ & $\begin{array}{r}6 \mathrm{NB} \\
06-6113^{4}\end{array}$ & $\begin{array}{rr}40 & 33 \\
106 & 17\end{array}$ & - & - & - & - & 6,510 & - & IR & $1910 a$ & $\begin{array}{l}\text { MacFarlane Irri- } \\
\text { gat1on Co }\end{array}$ & - & \\
\hline $\begin{array}{l}\text { Marshall Lake } \\
\text { South Boulder Creek } c\end{array}$ & $\begin{array}{r}60 \mathrm{H} \\
06-7300 \quad 05\end{array}$ & $\begin{array}{rr}39 & 57 \\
105 & 13\end{array}$ & - & 420 & - & - & 10,260 & - & IR & 1885 & $\begin{array}{l}\text { Farmers Reservoir } \\
\text { and Irrig Co }\end{array}$ & - & \\
\hline $\begin{array}{l}\text { Marston Lake } \\
\text { South Platte RIver and Bear } \\
\text { Creek c/ }\end{array}$ & 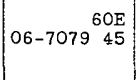 & $\begin{array}{rr}39 & 38 \\
105 & 04\end{array}$ & - & - & - & - & 19,790 & - & M & 1890 & C1ty of Denver & - & \\
\hline $\begin{array}{l}\text { Milton Lake } \\
\text { South Platte River } \mathfrak{c}\end{array}$ & $\begin{array}{r}60 G \\
06-7144 \\
45\end{array}$ & $\begin{array}{rr}40 & 14 \\
104 & 38\end{array}$ & - & 2,078 & - & - & 43,030 & - & IR & 1909 & \begin{tabular}{|} 
Farmers Reservo1r \\
and Irrigation Ca
\end{tabular} & - & \\
\hline $\begin{array}{l}\text { Model } \\
\text { Purgatoire River and Chicosa } \\
\text { Creek } \mathrm{c} \text { / }\end{array}$ & $07-1247^{7 \mathrm{AN}} 45$ & $\begin{array}{rr}37 & 20 \\
104 & 23\end{array}$ & - & 1,198 & - & - & 6,200 & - & I & $1909 a$ & $\begin{array}{l}\text { Model Land and } \\
\text { Irrigation Co }\end{array}$ & - & \\
\hline $\begin{array}{l}\text { Montgomery } \\
\text { Middle Fork South Platte River } c / \\
\text { See footnotes at end of table }\end{array}$ & $\begin{array}{r}60 \mathrm{~A} \\
06-6934 \quad 45\end{array}$ & $\begin{array}{rr}39 & 22 \\
106 & 04\end{array}$ & - & 97 & 0 & 5,090 & 5,090 & - & M & 1958 & Colorado Springs & - & $w_{\pi}$ \\
\hline
\end{tabular}




\begin{tabular}{|c|c|c|c|c|c|c|c|c|c|c|c|c|}
\hline \multirow[b]{2}{*}{$\begin{array}{l}\text { Name of reservolr } \\
\text { and } \\
\text { stream }\end{array}$} & \multicolumn{2}{|c|}{ Location } & \multirow{2}{*}{$\begin{array}{c}\text { Drainage } \\
\text { area } \\
\text { (square } \\
\text { miles) }\end{array}$} & \multirow[b]{2}{*}{$\begin{array}{c}\text { Surface } \\
\text { area } \\
\text { (acres) }\end{array}$} & \multicolumn{3}{|c|}{ Storage in acre-feet } & \multirow[b]{2}{*}{$\begin{array}{c}\text { Storage } \\
\text { rat10 }\end{array}$} & \multirow[b]{2}{*}{ Use } & \multirow[b]{2}{*}{$\begin{array}{c}\text { Date } \\
\text { com- } \\
\text { pleted }\end{array}$} & \multirow[b]{2}{*}{$\begin{array}{l}\text { Owner } \\
\text { or } \\
\text { operator }\end{array}$} & \multirow{2}{*}{$\begin{array}{l}\text { Storage } \\
\text { records } \\
\text { pub- } \\
\text { 21shed }\end{array}$} \\
\hline & \begin{tabular}{|c|} 
Basin \\
1ndex and \\
station no
\end{tabular} & $\begin{array}{l}\text { Lat1 tude } \\
\text { and } \\
\text { long1tude }\end{array}$ & & & Dead & Total & Usable & & & & & \\
\hline COLORADO--Continued & & & & & & & & & & & & \\
\hline $\begin{array}{l}\text { Mountain Home } \\
\text { Trinchera Creek }\end{array}$ & $\begin{array}{r}8 \mathrm{BE} \\
08-2410^{2}\end{array}$ & $\begin{array}{rr}37 & 23 \\
105 & 24\end{array}$ & a65 & - & - & - & 20,150 & 14 & I & $1915 \mathrm{a}$ & $\begin{array}{l}\text { Trinchera Irri- } \\
\text { gation Co }\end{array}$ & - \\
\hline $\begin{array}{l}\text { Narraguinnep } \\
\text { Delores RIver } \mathrm{c}\end{array}$ & $\mid \begin{array}{r}9 G \mathrm{GH} \\
09-3714 \quad 25\end{array}$ & $\begin{array}{rr}37 & 29 \\
108 & 37\end{array}$ & - & 581 & 200 & 9,300 & 9,100 & - & IR & 1908 & $\begin{array}{l}\text { Montezuma Valley } \\
\text { Irrigation Co }\end{array}$ & - \\
\hline $\begin{array}{l}\text { Nee Gronda } \\
\text { Arkansas River } \mathrm{c}\end{array}$ & $\begin{array}{r}7 \mathrm{AP} \\
07-1221 \quad 30\end{array}$ & $\begin{array}{rr}38 & 18 \\
102 & 46\end{array}$ & - & 3,572 & 39,860 & 98,660 & 58,800 & - & I & $1896 \mathrm{a}$ & $\begin{array}{l}\text { Amity Mutual Irri- } \\
\text { gation Co }\end{array}$ & - \\
\hline $\begin{array}{l}\text { Nee Noshe } \\
\text { Arkansas River } g \text { f }\end{array}$ & $07-1221 \quad 35$ & $\begin{array}{rr}38 & 20 \\
102 & 40\end{array}$ & - & 4,562 & 21,490 & 94,850 & 73,360 & - & I & $1896 \mathrm{a}$ & $\begin{array}{l}\text { Amity Mutual Irri- } \\
\text { gat1on Co }\end{array}$ & - \\
\hline $\begin{array}{l}\text { Nee Skah (Queen Dam) } \\
\text { Arkansas River c/ }\end{array}$ & $\begin{array}{r}7 \mathrm{AP} \\
07-1221 \quad 20\end{array}$ & $\begin{array}{rr}38 & 17 \\
102 & 38\end{array}$ & - & 1,930 & 9,940 & 35,660 & 25,720 & - & I & $1896 \mathrm{a}$ & $\begin{array}{l}\text { Amity Mutual Irr1- } \\
\text { gation Co }\end{array}$ & - \\
\hline $\begin{array}{l}\text { Nee Sopah } \\
\text { Arkansas River } c \text { f }\end{array}$ & \begin{tabular}{rr|} 
& $7 \mathrm{AP}$ \\
$07-1221$ & 25
\end{tabular} & $\begin{array}{rr}38 & 19 \\
102 & 46\end{array}$ & - & 3,628 & 10,920 & 36,400 & 25,500 & - & I & $1896 \mathrm{a}$ & $\begin{array}{l}\text { Amity Mutual Irri- } \\
\text { gation Co }\end{array}$ & - \\
\hline $\begin{array}{l}\text { North Poudre No } 5 \text { (Bee Lake) } \\
\text { North Fork Cache la Poudre } \\
\text { River c/ }\end{array}$ & $\begin{array}{r}60 \mathrm{~L} \\
06-7519\end{array}$ & $\begin{array}{rr}40 & 41 \\
105 & 02\end{array}$ & - & - & - & - & 8,410 & - & $I$ & 1894 & $\begin{array}{l}\text { North Poudre Irr1- } \\
\text { gation Co }\end{array}$ & - \\
\hline $\begin{array}{l}\text { North Poudre No } 6 \\
\text { North Fork Cache la Poudre } \\
\text { R1ver c/ }\end{array}$ & $\mid \begin{array}{r}60 \mathrm{~L} \\
06-7519\end{array}$ & $\begin{array}{rr}40 & 40 \\
105 & 02\end{array}$ & - & - & - & - & 9,990 & - & I & $1900 \mathrm{a}$ & $\begin{array}{l}\text { North Poudre Irri- } \\
\text { gation Co }\end{array}$ & - \\
\hline $\begin{array}{l}\text { North Poudre No } 15 \\
\text { Cache la Poudre Rfver and Dry } \\
\text { Creek } \mathrm{c} \text {. }\end{array}$ & $\begin{array}{r}60 L \\
06-7522 \quad 35\end{array}$ & $\begin{array}{rl}40 & 46 \\
105 & 07\end{array}$ & - & 312 & - & - & 5,530 & - & I & 1909 & $\begin{array}{l}\text { North Poudre Irri- } \\
\text { gation Co }\end{array}$ & - \\
\hline $\begin{array}{l}\text { Paonia } \\
\quad \text { Muddy Creek }\end{array}$ & \begin{tabular}{rr|}
$9 E D$ \\
$09-1314$ & 95
\end{tabular} & \begin{tabular}{rr|}
38 & 56 \\
107 & 21
\end{tabular} & 246 & - & 170 & 18,500 & 18,300 & - & IR & 1961 & $\begin{array}{l}\text { Bureau of } \\
\text { Reclamation }\end{array}$ & $\mathrm{PA}$ \\
\hline $\begin{array}{l}\text { Platoro } \\
\text { Conejos River }\end{array}$ & $08-2445 \quad 00$ & \begin{tabular}{rr|}
37 & 21 \\
106 & 33
\end{tabular} & a40 & 947 & 0 & 60,000 & 60,000 & 10 & FIR & 1951 & $\begin{array}{l}\text { Bureau of } \\
\text { Reclamation }\end{array}$ & PA \\
\hline $\begin{array}{l}\text { Point of Rocks } \\
\text { South Platte River and Cedar } \\
\text { Creek } \subseteq \text { / }\end{array}$ & $\mid \begin{array}{r}60 P \\
06-760325\end{array}$ & $\begin{array}{rr}40 & 47 \\
103 & 16\end{array}$ & - & 3,080 & 27,650 & 109,000 & 81,400 & - & IR & 1908 & $\begin{array}{l}\text { North Sterling } \\
\text { Irrigation Dist }\end{array}$ & - \\
\hline $\begin{array}{l}\text { Prewitt } \\
\text { South Platte River } c /\end{array}$ & $\begin{array}{r}60 \mathrm{OP} \\
06-7599 \quad 25\end{array}$ & $\begin{array}{rr}40 & 26 \\
103 & 22\end{array}$ & - & 2,430 & - & - & 32,820 & - & IR & 1912 & $\begin{array}{l}\text { Iliff Irrigation } \\
\text { District }\end{array}$ & - \\
\hline $\begin{array}{l}\text { Prospect } \\
\text { South Platte River } c \text { / }\end{array}$ & $\left|\begin{array}{rr}60 M \\
06-7568 & 25\end{array}\right|$ & $\begin{array}{rl}40 & 01 \\
104 & 30\end{array}$ & - & 400 & - & - & 5,610 & - & $I$ & 1911 & $\begin{array}{l}\text { Henrylen Irrigation } \\
\text { District }\end{array}$ & - \\
\hline
\end{tabular}


Ralston

Creek and South Boulder Creek c/

Reservolr No 8
Cache la Poudre RIver $c$ f

Rio Grande

R1vers1de

South Platte River c/

Sanchez

Ventero and Culebra Creeks

Santa Marla
North Clear Creek $\mathfrak{c}$ )

Sm1 th

Trinchera Creek

Standley Lake
South Platte River tributaries

Stillwater No 1

Sugar Loaf
Lake Fork c/

Taylor Park (Uncompangre)

Terrace

Alamosa Creek

Terry Lake Poudre R1ver and Dry Creek $\mathrm{c}$ )

Timnath Lake (Cache la Poudre Dam) (a) Poudre River of

Twin Lakes
Lake Creek

Two Buttes

Two Buttes Creek

Vallecito

Los Pinos (Pine) River

Vega

See footnotes at end of table

\begin{tabular}{|c|c|c|c|}
\hline$\left|\begin{array}{rr} & 60 F \\
06-7197 & 45\end{array}\right|$ & $\begin{array}{rr}39 & 50 \\
105 & 14\end{array}$ & - & 177 \\
\hline $\begin{array}{r}60 L \\
06-7519 \quad 55\end{array}$ & $\begin{array}{rr}40 & 39 \\
105 & 03\end{array}$ & - & - \\
\hline $\begin{array}{r}8 B A \\
08-2134 \quad 00\end{array}$ & $\begin{array}{rr}37 & 43 \\
107 & 17\end{array}$ & al6o & 1,030 \\
\hline $\begin{array}{r}60 \mathrm{M} \\
06-7566 \quad 25\end{array}$ & $\begin{array}{rr}40 & 20 \\
104 & 15\end{array}$ & - & 3,595 \\
\hline $\begin{array}{r}8 B F \\
08-2498 \quad 00\end{array}$ & $\begin{array}{rr}37 & 06 \\
105 & 25\end{array}$ & - & 2,660 \\
\hline $\begin{array}{r}8 \mathrm{BA} \\
08-215000\end{array}$ & $\begin{array}{rr}37 & 48 \\
107 & 06\end{array}$ & - & 580 \\
\hline $\mid \begin{array}{r}8 B E \\
08-2434 \\
25\end{array}$ & $\begin{array}{rr}37 & 22 \\
105 & 33\end{array}$ & a395 & 620 \\
\hline $\begin{array}{r}60 F \\
06-7207 \quad 45\end{array}$ & $\begin{array}{rr}39 & 52 \\
105 & 07\end{array}$ & - & 1,600 \\
\hline $09-2359 \quad 45$ & $\begin{array}{rr}40 & 02 \\
107 & 07\end{array}$ & - & - \\
\hline $07-0824 \quad 00$ & $\begin{array}{rr}39 & 15 \\
106 & 22\end{array}$ & - & 818 \\
\hline $09-1085$ 9EA & $\begin{array}{rr}38 & 49 \\
106 & 36\end{array}$ & 245 & 2,033 \\
\hline $\mid \begin{array}{r}8 B C \\
08-2364 \quad 00\end{array}$ & $\begin{array}{rr}37 & 21 \\
106 & 17\end{array}$ & 116 & 410 \\
\hline $\begin{array}{rr}60 \mathrm{~L} \\
06-7522 \quad 55\end{array}$ & $\begin{array}{rr}40 & 37 \\
105 & 05\end{array}$ & - & - \\
\hline $\begin{array}{rr}602 \\
06-7521 & 45\end{array}$ & 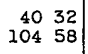 & - & - \\
\hline $07-0854 \quad \begin{array}{r}7 \mathrm{AA} \\
07\end{array}$ & $\begin{array}{rr}39 & 05 \\
106 & 19\end{array}$ & - & 2,273 \\
\hline $07-1348 \quad \begin{array}{r}7 \mathrm{AT} \\
45\end{array}$ & $\begin{array}{rr}37 & 38 \\
102 & 32\end{array}$ & - & 1,798 \\
\hline $09-3530$ OOA & $\begin{array}{rr}37 & 23 \\
107 & 34\end{array}$ & a270 & 2,720 \\
\hline $09-096100$ & $\begin{array}{rr}39 & 14 \\
107 & 49\end{array}$ & 244 & 910 \\
\hline
\end{tabular}

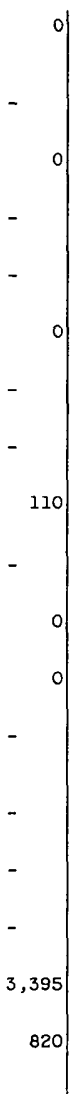

11,270
-
51,110
-
-
43,560
-
-
6,200
-
106,200
17,700
-
-
-
-

\begin{tabular}{r|c|c}
11,270 & - & IM \\
10,520 & - & $I$ \\
51,110 & 3 & IR \\
57,510 & - & I \\
103,000 & - & IR \\
43,560 & - & I \\
5,340 & 6 & I \\
17,660 & - & IR \\
6,100 & - & IR \\
17,370 & - & RW \\
106,200 & 8 & IR \\
17,700 & 2 & IPR \\
8,140 & - & IR \\
10,070 & - & I \\
54,450 & - & IMR \\
48,720 & - & IR \\
126,300 & - & IR \\
32,930 & - & IR \\
& - & -
\end{tabular}

1938a C1ty of Denver

1901 Windsor Reservo

1903a San Lu1s Valley

$1902 \begin{aligned} & \text { Riverside Reservo1r } \\ & \text { and Land Co }\end{aligned}$

1908 Sanchez Ditch a

1908a Sanchez Ditch an

1910a $\underset{\substack{\text { Santa Maria Reser- } \\ \text { vo1r Co }}}{\text {. }}$

1915a Trinchera Irr1

1902a Farmers Reservo1

and Irrigation

1940 Yampa Reservo1rs

1909 Colorado Fuel and 1937 Uncompahgre Valley

1904a Terrace Irrigation -

1890 Larimer and Weld Reservolr Co

1892 Cache 1a Poudre Reservolr $\mathrm{CO}$

1896a Tw1n Lakes Reser-
vo1r and Canal Co

1915a Two Buttes Water

Assoc

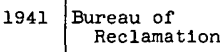

1960 Bureau of

Reclamation
Reau of 
Table 1 --Reservo1rs in the United States completed or under construction as of January 1, 1963, having a usable capac1ty of 5,000 acre-feet or more--Continued [For meaning of symbols under "Use" and "Storage records published," see text]

\begin{tabular}{|c|c|c|c|c|c|c|c|c|c|c|c|c|}
\hline \multirow[b]{2}{*}{$\begin{array}{l}\text { Name of reservo1r } \\
\text { and } \\
\text { s tream }\end{array}$} & \multicolumn{2}{|c|}{ Location } & \multirow{2}{*}{$\begin{array}{l}\text { Dralnage } \\
\text { area } \\
\text { (square } \\
\text { m1les) }\end{array}$} & \multirow[b]{2}{*}{$\begin{array}{c}\text { Surface } \\
\text { area } \\
\text { (acres) }\end{array}$} & \multicolumn{3}{|c|}{ Storage in acre-feet } & \multirow[b]{2}{*}{$\begin{array}{c}\text { storage } \\
\text { rat1o }\end{array}$} & \multirow[b]{2}{*}{ Use } & \multirow[b]{2}{*}{$\begin{array}{c}\text { Date } \\
\text { com- } \\
\text { pleted }\end{array}$} & \multirow[b]{2}{*}{$\begin{array}{l}\text { Owner } \\
\text { or } \\
\text { operator }\end{array}$} & \multirow{2}{*}{$\begin{array}{l}\text { Storage } \\
\text { records } \\
\text { pub- } \\
\text { lished }\end{array}$} \\
\hline & \begin{tabular}{|c|} 
Basin \\
1ndex and \\
station no
\end{tabular} & $\begin{array}{l}\text { Lat1tude } \\
\text { and } \\
\text { long1tude }\end{array}$ & & & Dead & Total & Usable & & & & & \\
\hline COLORADO--Cont1nued & & & & & & & & & & & & \\
\hline $\begin{array}{l}\text { W11liams Fork } \\
\text { W11liams Fork }\end{array}$ & $09-0380$ ODA & $\begin{array}{rr}40 & 02 \\
106 & 12\end{array}$ & 234 & 1,614 & 0 & 96,820 & 96,820 & 10 & IR & 1938 & $\begin{array}{l}\text { C1ty and County of } \\
\text { Denver }\end{array}$ & PA \\
\hline $\begin{array}{l}\text { Willow Creek } \\
\text { Willow Creek }\end{array}$ & $\mid \begin{array}{r}9 D A \\
09-0207\end{array}$ & $\begin{array}{rr}40 & 09 \\
105 & 56\end{array}$ & 134 & 303 & 1,490 & 10,550 & 9,060 & 4 & IR & 1953 & $\begin{array}{l}\text { Bureau of } \\
\text { Reclamation }\end{array}$ & $P A$ \\
\hline $\begin{array}{c}\text { Windsor } \\
\text { Cache la Poudre River } \mathrm{c} \\
\text { CONNECTICUT }\end{array}$ & $06-752125$ & $\begin{array}{rr}40 & 32 \\
104 & 53\end{array}$ & - & 1,000 & - & - & 17,690 & - & IR & 1890 & $\begin{array}{l}\text { Windsor Reservolr } \\
\text { and Canal Co }\end{array}$ & - \\
\hline $\begin{array}{l}\text { Barkhamsted (Saville Dam) } \\
\text { East Branch Farmington River }\end{array}$ & O1-1875 190 & $\begin{array}{ll}41 & 55 \\
72 & 57\end{array}$ & 538 & 2,320 & 28,760 & 97,470 & 68,710 & 9 & M & 1940 & $\begin{array}{l}\text { Metropolitan Dist } \\
\text { Comm, Hartford }\end{array}$ & PA \\
\hline $\begin{array}{l}\text { East Branch Compensating } \\
\text { East Branch Farmington River }\end{array}$ & $01-1876$ 1G0 & $\begin{array}{ll}41 & 53 \\
72 & 57\end{array}$ & 612 & 392 & 0 & 9,000 & 9,000 & - & $\mathrm{PR}$ & 1919 & $\begin{array}{l}\text { Metropol1tan Dist } \\
\text { Comm, Hartford }\end{array}$ & PA \\
\hline $\begin{array}{l}\text { Hall Meadow Brook } \\
\text { West Branch Naugatuck River }\end{array}$ & $01-2055^{1 \mathrm{HC}}$ & $\begin{array}{ll}41 & 52 \\
73 & 10\end{array}$ & 172 & 372 & - & 8,620 & - & - & $\mathrm{FR}$ & 1962 & $\begin{array}{l}\text { State Park and } \\
\text { Forest Commission }\end{array}$ & - \\
\hline $\begin{array}{l}\text { Hogback } \\
\text { West Branch Farmington River }\end{array}$ & $01-185900$ & $\begin{array}{ll}41 & 59 \\
73 & 01\end{array}$ & 122 & 550 & 0 & 20,000 & 20,000 & 1 & M & 1953 & $\begin{array}{l}\text { Metropolitan Dist } \\
\text { Comm, Hartford }\end{array}$ & - \\
\hline $\begin{array}{l}\text { Lake Candlewood (Rocky River Dam) } \\
\text { Rocky River }\end{array}$ & $01-2010^{1 \mathrm{HD}}$ & $\begin{array}{ll}41 & 35 \\
73 & 26\end{array}$ & $\begin{array}{ll}40 & 4\end{array}$ & 5,600 & 37,000 & 179,600 & 142,600 & - & $\mathrm{PR}$ & 1928 & $\begin{array}{l}\text { Connecticut Light } \\
\text { and Power Co }\end{array}$ & $\mathrm{PA}$ \\
\hline $\begin{array}{l}\text { Lake Ga1llard } \\
\text { Branford River }\end{array}$ & 1HA & $\begin{array}{ll}41 & 20 \\
72 & 46\end{array}$ & 173 & 1,150 & 15,360 & 48,630 & 33,270 & 20 & M & 1933 & New Haven Water co & - \\
\hline $\begin{array}{l}\text { Lake Lillinonah (Shepaug Dam) } \\
\text { Housatonic River }\end{array}$ & $01-2035$ 00 & $\begin{array}{ll}41 & 27 \\
73 & 18\end{array}$ & 1,391 & 1,870 & 33,000 & 74,000 & $b 5,038$ & $<05$ & $\mathrm{PR}$ & 1955 & $\begin{array}{l}\text { Connecticut Light } \\
\text { and Power Co }\end{array}$ & $\mathrm{PA}$ \\
\hline $\begin{array}{l}\text { Laurel } \\
\text { Mill (R1ppowam) RIver }\end{array}$ & $01-209790$ & $\begin{array}{ll}41 & 10 \\
73 & 33\end{array}$ & 128 & 265 & 300 & 6,910 & 6,610 & - & $M$ & 1924 & Stamford Water Co & - \\
\hline $\begin{array}{l}\text { Mad River } \\
\text { Mad R1ver }\end{array}$ & $\begin{array}{r}160 \\
01-186090\end{array}$ & $\begin{array}{ll}41 & 56 \\
73 & 06\end{array}$ & 182 & - & 200 & 9,700 & - & - & $\mathrm{FR}$ & 1962 & $\begin{array}{l}\text { State Park and } \\
\text { Forest Commission }\end{array}$ & - \\
\hline $\begin{array}{l}\text { Mansfield Hollow } \\
\text { Natchaug R1ver }\end{array}$ & $01-1215^{1 F H}$ & $\begin{array}{ll}41 & 45 \\
72 & 11\end{array}$ & 159 & 1,880 & $\mathrm{a} 40$ & 52,000 & 52,000 & 3 & $\mathrm{FR}$ & 1952 & Corps of Engineers & PA \\
\hline $\begin{array}{l}\text { Nepaug } \\
\text { Nepaug River and Phelps Brook }\end{array}$ & $01-187900$ & $\begin{array}{ll}41 & 50 \\
72 & 56\end{array}$ & 320 & 851 & 430 & 29,700 & 29,300 & 8 & M & 1918 & $\begin{array}{l}\text { Metropolitan Dist } \\
\text { Comm, Hartford }\end{array}$ & $\mathrm{PA}$ \\
\hline $\begin{array}{l}\text { Saugatuck } \\
\text { Saugatuck River }\end{array}$ & 01-2090 $1 \mathrm{HE}$ & $\begin{array}{ll}41 & 15 \\
73 & 21\end{array}$ & 35 & 868 & 21,370 & 38,000 & 16,600 & - & M & 1942 & $\begin{array}{l}\text { Br1dgeport Hydrau- } \\
\text { I10 Co }\end{array}$ & PA \\
\hline
\end{tabular}




\begin{tabular}{|c|c|c|c|c|c|c|c|c|c|c|c|c|}
\hline $\begin{array}{l}\text { Shen1psit Lake } \\
\text { Hockanum R1ver }\end{array}$ & $\mid \begin{array}{r}1 \mathrm{GP} \\
01-192000\end{array}$ & $\begin{array}{ll}41 & 52 \\
72 & 26\end{array}$ & 165 & 625 & a5, 100 & 14,400 & 29,300 & 5 & $M$ & 1871 & $\begin{array}{c}\text { Rockville water and } \\
\text { Aqueduct Co }\end{array}$ & PA \\
\hline $\begin{array}{l}\text { Thomaston } \\
\text { Naugatuck RIver } \\
\text { DELAWARE }\end{array}$ & $\mid \begin{array}{r}1 \mathrm{HC} \\
01-206600\end{array}$ & $\begin{array}{ll}41 & 42 \\
73 & 04\end{array}$ & 970 & 950 & of & 42,000 & - & - & $\mathrm{FR}$ & 1960 & Corps of Eng1neers & $\mathrm{PA}$ \\
\hline $\begin{array}{l}\text { Edgar Hoopes } \\
\text { Red Clay Creek tributary } \\
\text { FLORIDA }\end{array}$ & $\begin{array}{r}1 \mathrm{MKK} \\
01-479990\end{array}$ & $\begin{array}{ll}39 & 46 \\
75 & 38\end{array}$ & a2 & a200 & 0 & 6,750 & 6,750 & - & M & 1932 & City of Wilmington & - \\
\hline $\begin{array}{l}\text { Burrell lock and dam } \\
\text { Halnes Creek }\end{array}$ & $02-238000$ & $\begin{array}{ll}28 & 52 \\
81 & 47\end{array}$ & a640 & 106,000 & $>530,000$ & $>585,000$ & 55,000 & 18 & FNR & 1957 & \begin{tabular}{|} 
Oklawaha Basin Rec- \\
reation, Water \\
Control, and Con- \\
serv Author1ty
\end{tabular} & $\mathrm{PA}$ \\
\hline $\begin{array}{l}\text { Cherry Lake splliway } \\
\text { Palatlakaha Creek }\end{array}$ & $\begin{array}{r}2 M \\
02-236900\end{array}$ & $\begin{array}{ll}28 & 36 \\
81 & 49\end{array}$ & al6o & 25,600 & $>102,500$ & $>138,500$ & 36,000 & 42 & FNR & 1956 & $\begin{array}{l}\text { Oklawaha Basin Rec- } \\
\text { reation, water } \\
\text { Control, and Con- } \\
\text { serv Authority }\end{array}$ & PA \\
\hline $\begin{array}{l}\text { Conservation area No } 1 \\
\text { Everglades }\end{array}$ & $\begin{array}{r}20 \mathrm{~A} \\
02-281260\end{array}$ & $\begin{array}{ll}26 & 22 \\
80 & 20\end{array}$ & 221 & 141,400 & 0 & 292,000 & 292,000 & - & CFI & 1954 & $\begin{array}{l}\text { Central and Southern } \\
\text { Florida Flood } \\
\text { Control District }\end{array}$ & PA \\
\hline $\begin{array}{l}\text { Conservation area No } 2 \mathrm{~A} \\
\text { Everglades }\end{array}$ & $\begin{array}{r}20 \mathrm{~A} \\
02-284600\end{array}$ & $\begin{array}{ll}26 & 12 \\
80 & 27\end{array}$ & 151 & 96,600 & 0 & 363,000 & 363,000 & - & CFIR & 1954 & $\begin{array}{c}\text { Central and South- } \\
\text { ern Florida Flood } \\
\text { Control District }\end{array}$ & - \\
\hline $\begin{array}{c}\text { Conservation area No } 3 \mathrm{~A} \\
\text { Everglades }\end{array}$ & $\mid \begin{array}{r}20 \mathrm{~A} \\
02-289020\end{array}$ & $\begin{array}{ll}25 & 46 \\
80 & 45\end{array}$ & 787 & 503,700 & - & $2,726,000$ & 380,000 & - & $\mathrm{FR}$ & 1963 & $\begin{array}{c}\text { Central and South- } \\
\text { ern Florida Flood } \\
\text { Control District }\end{array}$ & - \\
\hline $\begin{array}{l}\text { Lake Apopka } \\
\text { Apopka-Beaucla1r Canal }\end{array}$ & $\begin{array}{r}2 M B \\
02-2376 \quad 00\end{array}$ & $\begin{array}{ll}28 & 44 \\
81 & 41\end{array}$ & al 80 & a31, 000 & - & - & - & - & FNR & 1956 & $\begin{array}{l}\text { Lake Apopka Recrea- } \\
\text { tion and Water } \\
\text { Conserv Control } \\
\text { Authority }\end{array}$ & PA \\
\hline $\begin{array}{l}\text { Lake Is tokpoga } \\
\text { Lake }\end{array}$ & $02-2717000$ & $\begin{array}{lll}\text { a } & 27 & 20 \\
\text { a } & 81 & 15\end{array}$ & 624 & 29,500 & b115,000 & 185,000 & 70,000 & 2 & FI & 1962 & $\begin{array}{c}\text { Central and South- } \\
\text { ern Florida Flood } \\
\text { Control D1strict }\end{array}$ & $\mathrm{PA}$ ef \\
\hline $\begin{array}{l}\text { Lake Okeechobee } \\
\text { Lake }\end{array}$ & $02-2550$ 2NF & $\begin{array}{lll}\text { a } & 26 & 57 \\
\text { a } & 80 & 50\end{array}$ & 25,650 & 450,000 & $b 2,900,000$ & $4,200,000$ & $1,300,000$ & 6 & FIN & 1938 & Corps of Engineers & $\mathrm{PA}$ ef \\
\hline $\begin{array}{l}\text { Lake Seminole (J1m Woodruff Dam)* } \\
\text { Apalach1cola RIver }\end{array}$ & $02-357500$ & $\begin{array}{ll}30 & 43 \\
84 & 52\end{array}$ & 17,150 & 41,560 & 680 & 426,600 & 425,900 & $<05$ & NPR & 1957 & Corps of Engineers & PA \\
\hline $\begin{array}{l}\text { Lake Talquin } \\
\text { Ochlockonee R1ver }\end{array}$ & $02-3299 \quad 00$ & $\begin{array}{ll}30 & 23 \\
84 & 39\end{array}$ & al, 660 & a10,000 & 0 & 73,000 & 73,000 & 06 & $P$ & 1930 & Flor1da Power Corp & PA \\
\hline $\begin{array}{l}\text { Moss Bluff } \\
\text { Oklawaha River }\end{array}$ & 2MD & $\begin{array}{ll}29 & 05 \\
81 & 53\end{array}$ & a910 & 20,500 & 130,000 & 177,500 & 47,500 & 09 & FN & 1925 & Corps of Engineers & - \\
\hline
\end{tabular}


Table 1 --Reservo1rs in the United States completed or under construction as of January 1, 1963, having a usable capacity of 5,000 acre-feet or more--Continued

[For meaning of symbols under "Use" and "Storage records published," see text]

\begin{tabular}{|c|c|c|c|c|c|c|c|c|c|c|c|c|}
\hline \multirow[b]{2}{*}{$\begin{array}{l}\text { Name of reservoir } \\
\text { and } \\
\text { stream }\end{array}$} & \multicolumn{2}{|c|}{ Location } & \multirow{2}{*}{$\begin{array}{l}\text { Drainage } \\
\text { area } \\
\text { (square } \\
\text { m1les) }\end{array}$} & \multirow[b]{2}{*}{$\begin{array}{c}\text { Surface } \\
\text { area } \\
\text { (acres) }\end{array}$} & \multicolumn{3}{|c|}{ Storage in acre-feet } & \multirow[b]{2}{*}{$\begin{array}{c}\text { Storage } \\
\text { rat10 }\end{array}$} & \multirow[b]{2}{*}{ Use } & \multirow[b]{2}{*}{$\begin{array}{c}\text { Date } \\
\text { com- } \\
\text { pleted }\end{array}$} & \multirow[b]{2}{*}{$\begin{array}{l}\text { Owner } \\
\text { or } \\
\text { operator }\end{array}$} & \multirow{2}{*}{$\begin{array}{l}\text { Storage } \\
\text { recorda } \\
\text { pub- } \\
\text { lished }\end{array}$} \\
\hline & $\begin{array}{l}\text { Basin } \\
\text { 1ndex and } \\
\text { station no }\end{array}$ & $\begin{array}{l}\text { Lat1 tude } \\
\text { and } \\
\text { long1 tude }\end{array}$ & & & Dead & Total & Usable & & & & & \\
\hline FLORIDA--Cont1nued & & & & & & & & & & & & \\
\hline $\begin{array}{l}\text { W1 thlacoochee } \\
\text { W1 thlacoochee River } \\
\text { GEORGIA }\end{array}$ & $02-313220$ & $\begin{array}{ll}29 & 01 \\
82 & 37\end{array}$ & 21,980 & 4,163 & - & - & 15,330 & $<005$ & $\mathrm{P}$ & $1905 a$ & Flor1da Power Corp & - \\
\hline $\begin{array}{l}\text { Allatoona } \\
\text { Etowah River }\end{array}$ & $\begin{array}{r}2 \mathrm{VF} \\
02-3935 \text { OD }\end{array}$ & $\begin{array}{ll}34 & 10 \\
84 & 44\end{array}$ & 1,110 & 19,200 & 182,800 & $, 670,000$ & 587,200 & 5 & $\mathrm{FPR}$ & 1949 & Corps of Engineers & $\mathrm{PA}$ \\
\hline $\begin{array}{l}\text { Blue Ridge Lake } \\
\text { Toccoa RIver }\end{array}$ & $03-558500$ & $\begin{array}{ll}34 & 53 \\
84 & 17\end{array}$ & 232 & 3,320 & b14,500 & 200,800 & $\mathrm{~b} 186,300$ & 42 & $P R$ & 1931 & $\begin{array}{l}\text { Tennessee Valley } \\
\text { Authority }\end{array}$ & $P A, P E$ \\
\hline $\begin{array}{l}\text { Burton } \\
\text { Tallulah R1ver }\end{array}$ & $02-1785^{2 J 8} 00$ & $\begin{array}{ll}34 & 47 \\
83 & 32\end{array}$ & 115 & 2,775 & 21,000 & 108,000 & 87,000 & 5 & $\mathrm{PR}$ & 1920 & Georgla Power Co & $\mathrm{PA}$ \\
\hline $\begin{array}{l}\text { Clark Hill }{ }^{*} \\
\text { Savannah R1ver }\end{array}$ & $02-1945$ OO & $\begin{array}{ll}33 & 40 \\
82 & 12\end{array}$ & 6,150 & 78,500 & $1,170,000$ & $2,900,000$ & $1,730,000$ & 6 & FNP & 1952 & Corps of Engineers & $\mathrm{PA}$ \\
\hline $\begin{array}{l}\text { Columbia } \\
\text { Chattahoochee River }\end{array}$ & $02-3438^{2 R H} 00$ & $\begin{array}{ll}31 & 15 \\
85 & 07\end{array}$ & 8,210 & 1,570 & 590 & 18,170 & 17,580 & $<05$ & NPR & 1963 & Corps of Engineers & - \\
\hline $\begin{array}{l}\text { Flint River } \\
\text { Flint RIver }\end{array}$ & $02-3505^{2 S D}$ & $\begin{array}{ll}31 & 36 \\
84 & 08\end{array}$ & 4,180 & 2,500 & - & - & 7,500 & $<05$ & PR & 1921 & Georg1a Power Co & - \\
\hline $\begin{array}{l}\text { Goat Rock* } \\
\text { Chattahoochee River }\end{array}$ & $02-3413^{2 R E} 00$ & $\begin{array}{ll}32 & 36 \\
85 & 05\end{array}$ & 4,520 & 940 & 6,000 & all,, 000 & a5, 000 & $<05$ & $\mathrm{PR}$ & 1912 & Georgla Power Co & - \\
\hline $\begin{array}{l}\text { Hartwell * } \\
\text { Savannah River }\end{array}$ & $02-187250$ & $\begin{array}{ll}34 & 21 \\
82 & 50\end{array}$ & 2,088 & 61,350 & $1,134,100$ & $2,842,700$ & $1,708,600$ & 6 & FNP & 1961 & Corps of Engineers & PA \\
\hline $\begin{array}{l}\text { Lake Harding (Bartletts Ferry Dam)* } \\
\text { Chattahoochee River }\end{array}$ & $02-3410 \quad 00$ & $\begin{array}{ll}32 & 40 \\
85 & 05\end{array}$ & 4,240 & 5,850 & 124,000 & 181,000 & b57,000 & $<05$ & MNPRW & 1926 & Georgla Power Co & $\mathrm{PA}$ \\
\hline $\begin{array}{l}\text { Lake Seminole (J1m Woodruff)* } \\
\text { Apalachicola R1ver }\end{array}$ & $02-3575^{2 \mathrm{SH}}$ & $\begin{array}{ll}30 & 43 \\
84 & 52\end{array}$ & a.17,100 & 41,560 & 680 & 426,600 & 425,900 & $<05$ & NPR & 1957 & Corps of Engineers & PA \\
\hline $\begin{array}{l}\text { Lake S1dney Lanier (Buford Dam) } \\
\text { Chat tahoochee River }\end{array}$ & $22-334400$ & $\begin{array}{ll}34 & 10 \\
84 & 04\end{array}$ & 1,040 & 47,182 & 867,600 & $2,554,000$ & $1,686,400$ & 10 & FMPR & 1957 & Corps of Engineers & PA \\
\hline $\begin{array}{l}\text { Lloyd Shoals } \\
\text { Ocmulgee River }\end{array}$ & $02-2100 \mathrm{OKF}$ & $\begin{array}{ll}33 & 19 \\
83 & 50\end{array}$ & 1,400 & 4,750 & 63,000 & 107,000 & 44,000 & 07 & $\mathrm{PR}$ & 1911 & Georgla Power Co & $\mathrm{PA}$ \\
\hline $\begin{array}{l}\text { Math1s (Terrora-Rabun) } \\
\text { Tallulah River }\end{array}$ & $02-179500$ & $\begin{array}{ll}34 & 46 \\
83 & 25\end{array}$ & 151 & 834 & 8,000 & 31,000 & 23,000 & 08 & PR & 1914 & Georgia Power co & $\mathrm{PA}$ \\
\hline $\begin{array}{l}\text { Nottely Lake } \\
\text { Nottely River }\end{array}$ & $03-553000$ & $\begin{array}{ll}34 & 57 \\
84 & 05\end{array}$ & 214 & 4,290 & 13,200 & 184,400 & 171,200 & $\epsilon$ & FNPR & 1942 & $\begin{array}{l}\text { Tennessee Valley } \\
\text { Author1ty }\end{array}$ & $P A, P E$ \\
\hline
\end{tabular}




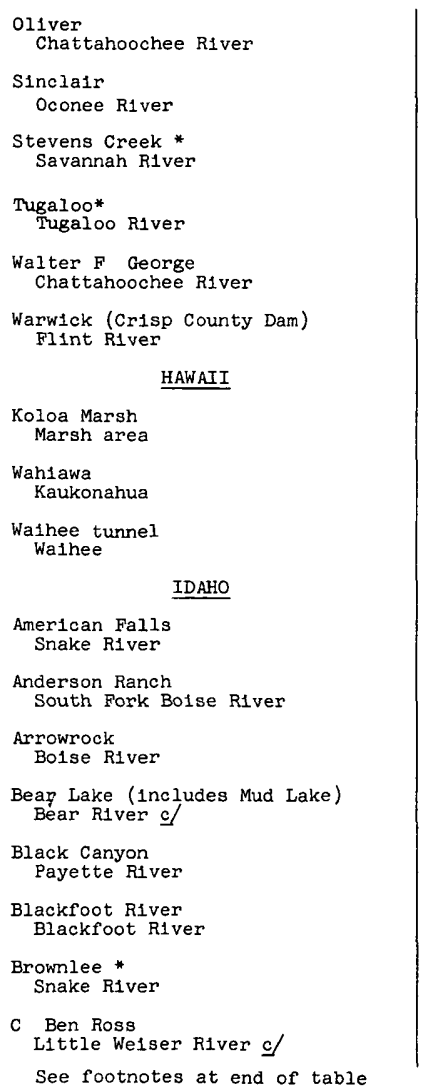

\begin{tabular}{|c|c|c|c|}
\hline $\begin{array}{r}2 \mathrm{RE} \\
02-341400\end{array}$ & $\begin{array}{ll}32 & 30 \\
85 & 00\end{array}$ & 4,630 & 2,150 \\
\hline $\begin{array}{r}2 K C \\
02-222500\end{array}$ & $\begin{array}{ll}33 & 10 \\
83 & 14\end{array}$ & 2,900 & 15,350 \\
\hline $02-1964 \begin{array}{r}2 \mathrm{JJ} \\
50\end{array}$ & $\begin{array}{ll}33 & 34 \\
82 & 03\end{array}$ & 7,180 & 4,300 \\
\hline $\begin{array}{r}2 \mathrm{JB} \\
02-1816 \quad 00\end{array}$ & $\begin{array}{ll}34 & 41 \\
83 & 21\end{array} \mid$ & 465 & 597 \\
\hline $02-3432 \quad 40$ & $\begin{array}{ll}31 & 38 \\
85 & 04\end{array}$ & 7,460 & 48,600 \\
\hline $\begin{array}{r}2 S D \\
02-3504 \quad 00\end{array}$ & $\begin{array}{ll}31 & 51 \\
83 & 57\end{array}$ & 3,750 & 7,000 \\
\hline $40-0527$ oo & $\begin{array}{rr}21 & 55 \\
159 & 27\end{array}$ & 24 & 424 \\
\hline $40-2095$ oo & $\begin{array}{rr}21 & 30 \\
158 & 03\end{array}$ & 167 & 302 \\
\hline $40-2837 \quad 50$ & $\begin{array}{rr}21 & 27 \\
157 & 52\end{array}$ & - & - \\
\hline $\begin{array}{r}13 \mathrm{EN} \\
13-076500\end{array}$ & $\begin{array}{rr}42 & 47 \\
112 & 53\end{array}$ & 13,580 & 56,100 \\
\hline $\begin{array}{r}13 D D \\
13-1900 \text { DD }\end{array}$ & $\begin{array}{rr}43 & 22 \\
115 & 27\end{array}$ & 980 & 4,780 \\
\hline $\begin{array}{r}13 \mathrm{DD} \\
13-1940 \quad 00\end{array}$ & $\begin{array}{rr}43 & 36 \\
115 & 55\end{array}$ & 2,210 & 3,050 \\
\hline $\begin{array}{r}10 \mathrm{HB} \\
10-055500\end{array}$ & $\begin{array}{rr}42 & 07 \\
111 & 19\end{array}$ & - & 87,000 \\
\hline$-^{13 D G}$ & $\begin{array}{rr}43 & 56 \\
116 & 26\end{array}$ & 2,680 & 1,045 \\
\hline $\begin{array}{r}13 \mathrm{EK} \\
13-065000\end{array}$ & $\begin{array}{rr}43 & 00 \\
111 & 43\end{array}$ & 581 & 17,300 \\
\hline $\begin{array}{r}13 \mathrm{DK} \\
13-289700\end{array}$ & $\begin{array}{rr}44 & 50 \\
116 & 54\end{array}$ & 72,590 & 15,100 \\
\hline${ }_{-}^{13 D F}$ & $\begin{array}{rr}44 & 31 \\
116 & 26\end{array}$ & - & 353 \\
\hline
\end{tabular}

\begin{tabular}{|c|c|c|c|c|c|c|}
\hline 26,000 & 32,000 & 6,000 & $<05$ & MNPRW & 1959 & Georgia Power Co \\
\hline 25,000 & 334,000 & 109,000 & 09 & MPR & 1953 & Georgla Power co \\
\hline 5,000 & 16,000 & 11,000 & $<05$ & $P$ & 1914 & $\begin{array}{l}\text { South Carolina Elec- } \\
\text { tric and Gas Co }\end{array}$ \\
\hline 31,500 & 43,000 & all, 500 & $<05$ & $\mathrm{PR}$ & 1923 & Georg1a Power co \\
\hline 196,700 & $1,028,100$ & 831,400 & 1 & NPR & 1963 & Corps of Engineers \\
\hline 114,000 & 149,000 & a35,000 & $<05$ & MPR & 1930 & Crisp County \\
\hline 430 & 7,686 & 7,256 & - & I & 1905 & Grove Farm co Inc \\
\hline 307 & 9,329 & 9,022 & 25 & I & 1905 & $\begin{array}{l}\text { Walalua Agricul- } \\
\text { tural Co, Ltd }\end{array}$ \\
\hline 0 & 6,660 & 6,660 & - & $\mathrm{M}$ & 1955 & $\begin{array}{l}\text { Honolulu Board of } \\
\text { Water Supply }\end{array}$ \\
\hline 0 & $1,700,000$ & $1,700,000$ & 3 & IP & 1926 & $\begin{array}{l}\text { Bureau of } \\
\text { Reclamation }\end{array}$ \\
\hline 29,000 & 493,200 & 464,200 & 7 & FIP & 1950 & $\begin{array}{l}\text { Bureau of } \\
\text { Reclamation }\end{array}$ \\
\hline 0 & 286,600 & 286,600 & 2 & I & 1915 & $\begin{array}{l}\text { Bureau of } \\
\text { Reclamation }\end{array}$ \\
\hline - & - & $1,420,000$ & - & IPR & 1914 & $\begin{array}{l}\text { Utah Power and } \\
\text { Light Co }\end{array}$ \\
\hline 200 & 44,100 & 43,900 & $<05$ & IP & 1924 & $\begin{array}{l}\text { Bureau of } \\
\text { Reclamation }\end{array}$ \\
\hline - & $\mathrm{d} 413,000$ & bd 350,000 & - & I & 1924 & $\begin{array}{l}\text { Bureau of Indian } \\
\text { Affa1rs }\end{array}$ \\
\hline 426,700 & $1,426,700$ & $1,000,000$ & - & NP & 1958 & Idaho Power Co \\
\hline 0 & 7,787 & 7,787 & - & I & 1936 & $\begin{array}{l}\text { Little We1ser River } \\
\text { Irrigation Dist }\end{array}$ \\
\hline
\end{tabular}



[For meaning of symbols under "Use" and "Storage records published," see text]

\begin{tabular}{|c|c|c|c|c|c|c|c|c|c|c|c|c|}
\hline \multirow[b]{2}{*}{$\begin{array}{l}\text { Name of reservo1r } \\
\text { and } \\
\text { stream }\end{array}$} & \multicolumn{2}{|c|}{ Location } & \multirow{2}{*}{$\begin{array}{l}\text { Dra1nage } \\
\text { area } \\
\text { (square } \\
\text { m1les) }\end{array}$} & \multirow[b]{2}{*}{$\begin{array}{c}\text { Surface } \\
\text { area } \\
\text { (acres) }\end{array}$} & \multicolumn{3}{|c|}{ Storage in acre-feet } & \multirow[b]{2}{*}{$\begin{array}{c}\text { Storage } \\
\text { rat10 }\end{array}$} & \multirow[b]{2}{*}{ Use } & \multirow[b]{2}{*}{$\begin{array}{c}\text { Date } \\
\text { com- } \\
\text { pleted }\end{array}$} & \multirow[b]{2}{*}{$\begin{array}{l}\text { Owner } \\
\text { or } \\
\text { operator }\end{array}$} & \multirow{2}{*}{$\begin{array}{l}\text { Storage } \\
\text { records } \\
\text { pub- } \\
\text { lished }\end{array}$} \\
\hline & \begin{tabular}{|c|} 
Basin \\
1ndex and \\
station no
\end{tabular} & \begin{tabular}{|l|} 
Lat1tude \\
and \\
long1tude
\end{tabular} & & & Dead & Total & Usable & & & & & \\
\hline IDAHO--ContInued & & & & & & & & & & & & \\
\hline C $\begin{array}{c}J \text { Strike } \\
\text { Snake River }\end{array}$ & $\begin{array}{r}13 \mathrm{DC} \\
13-171500\end{array}$ & $\begin{array}{rr}42 & 57 \\
115 & 59\end{array}$ & - & 7,500 & 166,000 & 250,000 & 84,000 & $<\begin{array}{ll}0 & 05\end{array}$ & $P$ & 1952 & Idaho Power Co & PA \\
\hline $\begin{array}{l}\text { Cabinet Gorge } \\
\text { Clark Fork R1ver }\end{array}$ & $-^{12 \mathrm{DG}}$ & $\begin{array}{rr}47 & 05 \\
116 & 03\end{array}$ & 21,840 & 3,228 & - & - & 43,500 & $<05$ & $\mathrm{P}$ & 1952 & $\begin{array}{l}\text { Washington water } \\
\text { Power Co }\end{array}$ & - \\
\hline $\begin{array}{l}\text { Cascade } \\
\quad \text { North Fork Payette R1ver }\end{array}$ & $\begin{array}{r}13 \mathrm{DG} \\
13-244500\end{array}$ & \begin{tabular}{rr|}
44 & 32 \\
116 & 03
\end{tabular} & 626 & 26,500 & 50,000 & 703,200 & 653,200 & 9 & IP & 1949 & $\begin{array}{l}\text { Bureau of } \\
\text { Reclamation }\end{array}$ & PA \\
\hline $\begin{array}{l}\text { Cedar Creek } \\
\text { Cedar Creek }\end{array}$ & $\begin{array}{r}13 \mathrm{ES} \\
13-106700\end{array}$ & $\begin{array}{rr}42 & 13 \\
114 & 53\end{array}$ & 128 & - & - & 26,000 & - & - & $I$ & 1920 & Cedar Mesa Co & PA \\
\hline $\begin{array}{l}\text { Coeur d'Alene Lake } \\
\text { Spokane R1ver }\end{array}$ & $\begin{array}{r}12 \mathrm{IEG} \\
12-4155^{2} 00\end{array}$ & \begin{tabular}{rr|}
47 & 42 \\
116 & 58
\end{tabular} & 3,700 & 32,000 & 21,400 & 238,500 & 217,100 & 5 & $P$ & 1906 & $\begin{array}{l}\text { washington water } \\
\text { Power Co }\end{array}$ & PA \\
\hline $\begin{array}{l}\text { Crane Creek } \\
\text { Crane Creek }\end{array}$ & $\begin{array}{r}13 D F \\
13-264000\end{array}$ & $\begin{array}{rr}44 & 22 \\
116 & 37\end{array}$ & 242 & $a 3,300$ & 0 & $a 60,000$ & $a 60,000$ & 12 & I & 1921 & $\begin{array}{l}\text { Crane Creek Reser- } \\
\text { volr Admin1stra- } \\
\text { t1on Board }\end{array}$ & $\mathrm{PA}$ ef \\
\hline $\begin{array}{l}\text { Deadwood } \\
\text { Deadwood River }\end{array}$ & $\begin{array}{r}13 \mathrm{DG} \\
13-236000\end{array}$ & $\begin{array}{rr}44 & 18 \\
115 & 39\end{array}$ & 112 & 3,160 & 1,500 & 161,900 & 160,400 & 11 & CIP & 1930 & $\begin{array}{l}\text { Bureau of } \\
\text { Reclamation }\end{array}$ & $\mathrm{PA}$ ef \\
\hline $\begin{array}{l}\text { Deep Creek (Henderson Dam) } \\
\text { Deep Creek }\end{array}$ & $-^{10 \mathrm{HD}}$ & $\begin{array}{rr}42 & 13 \\
112 & 10\end{array}$ & - & 181 & 0 & $a 5,400$ & a5, 400 & 8 & I & 1951 & $\begin{array}{l}\text { Deep Creek Irr1- } \\
\text { gation Co }\end{array}$ & - \\
\hline $\begin{array}{l}\text { Deep Creek } \\
\text { Deep Creek and springs }\end{array}$ & ${ }_{-}^{10 \mathrm{HA}}$ & $\begin{array}{rr}42 & 04 \\
112 & 41\end{array}$ & - & - & - & 6,594 & - & - & I & 1948 & $\begin{array}{l}\text { Pratt Irrigation } \\
\text { Co, Ltd }\end{array}$ & - \\
\hline $\begin{array}{l}\text { F1sh Creek } \\
\text { Fish Creek }\end{array}$ & $-13 \mathrm{ET}$ & $\begin{array}{rr}43 & 25 \\
113 & 49\end{array}$ & 628 & 558 & of & 14,411 & 14,411 & 13 & I & 1922 & $\begin{array}{l}\text { Carey Valley } \\
\text { Reservolr Co }\end{array}$ & - \\
\hline $\begin{array}{l}\text { Glendale } \\
\text { Cub River } c /\end{array}$ & $\begin{array}{r}10 \mathrm{HC} \\
10-098510\end{array}$ & $\begin{array}{rr}42 & 08 \\
111 & 44\end{array}$ & - & 220 & 0 & 5,750 & 5,750 & - & I & 1928 & $\begin{array}{r}\text { Preston-Whitney } \\
\text { Irrigation Co }\end{array}$ & - \\
\hline $\begin{array}{l}\text { Grays Lake } \\
\text { Willow Creek }\end{array}$ & $-13 \mathrm{EJ}$ & $\begin{array}{rr}43 & 10 \\
111 & 32\end{array}$ & - & 22,000 & 360,000 & 400,000 & 40,000 & - & I & 1924 & $\begin{array}{l}\text { Bureau of Indian } \\
\text { Affairs }\end{array}$ & PA \\
\hline $\begin{array}{l}\text { Henrys Lake } \\
\text { Henrys Fork }\end{array}$ & $\begin{array}{r}13 \mathrm{ER} \\
13-039000\end{array}$ & $\begin{array}{rr}44 & 36 \\
111 & 21\end{array}$ & 98 & 6,356 & - & - & 79,351 & 23 & I & 1923 & $\begin{array}{l}\text { North Fork Reser- } \\
\text { vo1r Co }\end{array}$ & PA \\
\hline $\begin{array}{l}\text { Hubbard } \\
\text { Boise River } c \text { f }\end{array}$ & $-130 D$ & $\begin{array}{rr}43 & 31 \\
116 & 21\end{array}$ & - & - & - & - & 7,500 & - & I & 1906 & $\begin{array}{l}\text { Bureau of } \\
\text { Reclamation }\end{array}$ & - \\
\hline $\begin{array}{l}\text { Is land Park } \\
\text { Henrys Fork }\end{array}$ & $13-042000$ & $\begin{array}{rr}44 & 25 \\
111 & 24\end{array}$ & 481 & 7,794 & 0 & 127,265 & 127,265 & 3 & I & 1938 & $\begin{array}{l}\text { Bureau of } \\
\text { Reclamation }\end{array}$ & $\mathrm{PA}$ \\
\hline
\end{tabular}




\begin{tabular}{|c|c|c|c|c|c|c|c|c|c|c|c|c|}
\hline $\begin{array}{l}\text { Lake Fork } \\
\text { Lake Fork Payette River }\end{array}$ & $\left|\begin{array}{r}13 D G \\
13-241000\end{array}\right|$ & $\begin{array}{rr}44 & 54 \\
116 & 03\end{array}$ & 64 & 1,500 & - & - & $\mathrm{d} 16,940$ & 2 & I & 1926 & $\begin{array}{l}\text { Lake Irrigation } \\
\text { Distr1ct }\end{array}$ & PA \\
\hline $\begin{array}{l}\text { Lake Lowell (Deer Flat Dam) } \\
\text { Bo1se RIver and Moore Creek } c /\end{array}$ & $\begin{array}{r}13 \mathrm{DD} \\
13-203500\end{array}$ & $\begin{array}{rr}43 & 35 \\
116 & 45\end{array}$ & - & 9,835 & 13,960 & 190,100 & 176,140 & - & I & 1908 & $\begin{array}{l}\text { Bureau of } \\
\text { Reclamation }\end{array}$ & PA \\
\hline $\begin{array}{l}\text { Lake Waha } \\
\text { Lake Creek }\end{array}$ & $-^{13 B D}$ & $\begin{array}{rr}46 & 13 \\
116 & 52\end{array}$ & - & - & - & 6,900 & - & - & I & 1915 & $\begin{array}{l}\text { Bureau of } \\
\text { Reclamation }\end{array}$ & - \\
\hline $\begin{array}{l}\text { Lake Walcott } \\
\text { Snake River }\end{array}$ & $\begin{array}{r}13 E P \\
13-081000\end{array}$ & $\begin{array}{rr}42 & 40 \\
113 & 29\end{array}$ & 15,700 & 12,250 & 115,000 & 210,180 & $b 95,180$ & $<05$ & PI & 1906 & $\begin{array}{l}\text { Bureau of } \\
\text { Reclamation }\end{array}$ & PA \\
\hline $\begin{array}{l}\text { Little Camas } \\
\text { L1ttle Camas Creek }\end{array}$ & $-13 \mathrm{DD}$ & $\begin{array}{rr}43 & 21 \\
115 & 23\end{array}$ & $\begin{array}{ll}31 & 8\end{array}$ & 1,250 & - & - & 22,300 & - & I & 1918 & $\begin{array}{l}\text { Mountain Home Irr1- } \\
\text { gation District }\end{array}$ & PA \\
\hline $\begin{array}{l}\text { Little Wood } \\
\text { L1ttle Wood R1ver }\end{array}$ & $\begin{array}{r}13 \mathrm{ET} \\
13-148200\end{array}$ & $\begin{array}{rr}43 & 25 \\
114 & 02\end{array}$ & 279 & 571 & 8 & 29,960 & 29,950 & 3 & I & 1959 & $\begin{array}{l}\text { Little Wood River } \\
\text { Irrigation Dist }\end{array}$ & PA \\
\hline $\begin{array}{l}\text { Lost Valley } \\
\text { Lost Creek }\end{array}$ & $\begin{array}{r}13 \mathrm{DF} \\
13-254000\end{array}$ & $\begin{array}{rr}44 & 57 \\
116 & 28\end{array}$ & 294 & 700 & of & 7,140 & 7,140 & 3 & I & 1938 & $\begin{array}{l}\text { Lost Valley Reser- } \\
\text { volr Co }\end{array}$ & $\mathrm{PA}$ ef \\
\hline $\begin{array}{l}\text { Lower Salmon Falls } \\
\text { Snake R1ver }\end{array}$ & $-13 \mathrm{ET}$ & $\begin{array}{rr}42 & 51 \\
114 & 54\end{array}$ & - & a840 & - & - & a5,200 & $<05$ & $P$ & 1949 & Idaho Power Co & - \\
\hline $\begin{array}{l}\text { Lucky Peak } \\
\text { Bo1se River }\end{array}$ & $\mid \begin{array}{r}13 D D \\
13-201500\end{array}$ & $\begin{array}{rr}43 & 32 \\
116 & 04\end{array}$ & 22,680 & 3,810 & 28,770 & 307,040 & 278,270 & 1 & FIR & 1955 & Corps of Englneers & PA \\
\hline $\begin{array}{l}\text { Mackay } \\
\text { Big Lost River }\end{array}$ & $\begin{array}{r}13 \mathrm{EL} \\
13-126000\end{array}$ & $\begin{array}{rr}43 & 57 \\
113 & 40\end{array}$ & 788 & 1,360 & a.25 & 44,495 & $a 44,370$ & 2 & I & 1956 & $\begin{array}{l}\text { Big Lost River } \\
\text { Irrigation Dist }\end{array}$ & PA \\
\hline $\begin{array}{l}\text { Magic } \\
\text { B1g Wood River }\end{array}$ & $\begin{array}{r}13 \mathrm{ET} \\
13-1420 \text { OO }\end{array}$ & \begin{tabular}{rr|}
43 & 15 \\
114 & 22
\end{tabular} & al, 600 & 3,904 & - & - & d191,500 & 6 & I & 1917 & Big Wood Canal Co & PA \\
\hline $\begin{array}{l}\text { Mud Lake } \\
\text { Camas Creek }\end{array}$ & $\begin{array}{r}13 E J \\
13-1150\end{array}$ & $\begin{array}{rr}43 & 54 \\
112 & 21\end{array}$ & 1,130 & $a 6,600$ & - & 61,660 & - & - & I & 1914 & $\begin{array}{l}\text { Owsley Canal Co, } \\
\text { and others }\end{array}$ & PA \\
\hline $\begin{array}{l}\text { Oakley } \\
\text { Goose Creek and diversion }\end{array}$ & $\begin{array}{r}13 \mathrm{EP} \\
13-083500\end{array}$ & $\begin{array}{rr}42 & 12 \\
113 & 55\end{array}$ & 729 & 1,250 & 0 & 74,350 & 74,350 & 2 & I & 1913 & Oakley Canal Co & PA \\
\hline $\begin{array}{l}\text { Onelda } \\
\text { Bear River }\end{array}$ & $10-086400$ & $\begin{array}{rr}42 & 16 \\
111 & 45\end{array}$ & 4,400 & 480 & - & - & 11,500 & $<05$ & FIP & 1913 & $\begin{array}{l}\text { Utah Power and } \\
\text { Light Co }\end{array}$ & - \\
\hline $\begin{array}{l}\text { Oxbow * } \\
\text { Snake RIver }\end{array}$ & $-13 \mathrm{DK}$ & $\begin{array}{rr}44 & 58 \\
116 & 51\end{array}$ & 73,150 & - & - & 52,500 & - & - & $\mathrm{P}$ & 1961 & Idaho Power Co & - \\
\hline $\begin{array}{l}\text { Paddock Valley } \\
\text { L1ttle W1llow Creek }\end{array}$ & $-13 D G$ & $\begin{array}{rr}44 & 12 \\
116 & 36\end{array}$ & 28 & al,, 500 & 0 & a32,000 & $a 32,000$ & - & I & 1950 & $\begin{array}{l}\text { Little willow Creek } \\
\text { Irrigation Co }\end{array}$ & - \\
\hline $\begin{array}{l}\text { Palisades } \\
\text { Snake River }\end{array}$ & $\begin{array}{r}13 E G \\
13-0324 \quad 50\end{array}$ & $\begin{array}{rr}43 & 20 \\
111 & 12\end{array}$ & 5,208 & 15,150 & 199,600 & $1,401,600$ & $1,202,000$ & 3 & CFIMP & 1955 & $\begin{array}{l}\text { Bureau of } \\
\text { Reclamation }\end{array}$ & PA \\
\hline $\begin{array}{l}\text { Payette Lake } \\
\text { North Fork Payette River }\end{array}$ & $\begin{array}{rl}13 D G \\
13-2385 & 00\end{array} \mid$ & $\begin{array}{rr}44 & 55 \\
116 & 07\end{array}$ & 144 & 5,000 & - & - & $\mathrm{a} 27,700$ & 1 & I & 1943 & Lake Reservoir Co & $\mathrm{PA}$ ef \\
\hline $\begin{array}{l}\text { Pend Orelile Lake } \\
\text { Pend Orellle River }\end{array}$ & $\begin{array}{r}12 D M \\
12-392500\end{array}$ & $\begin{array}{rr}48 & 11 \\
117 & 00\end{array}$ & 22,900 & 94,600 & 406,200 & $1,561,300$ & $1,155,100$ & 06 & CFNPR & 1952 & Corps of Engineers & PA \\
\hline
\end{tabular}




\begin{tabular}{|c|c|c|c|c|c|c|c|c|c|c|c|c|}
\hline \multirow[b]{2}{*}{$\begin{array}{l}\text { Name of reservolr } \\
\text { and } \\
\text { stream }\end{array}$} & \multicolumn{2}{|c|}{ Location } & \multirow{2}{*}{$\begin{array}{c}\text { Drainage } \\
\text { area } \\
\text { (square } \\
\text { m1les) }\end{array}$} & \multirow[b]{2}{*}{$\begin{array}{c}\text { Surface } \\
\text { area } \\
\text { (acres) }\end{array}$} & \multicolumn{3}{|c|}{ Storage in acre-feet } & \multirow[b]{2}{*}{$\begin{array}{c}\text { Storage } \\
\text { rat1o }\end{array}$} & \multirow[b]{2}{*}{ Use } & \multirow[b]{2}{*}{$\begin{array}{c}\text { Date } \\
\text { com- } \\
\text { pleted }\end{array}$} & \multirow[b]{2}{*}{$\begin{array}{l}\text { Owner } \\
\text { or } \\
\text { operator }\end{array}$} & \multirow{2}{*}{$\begin{array}{l}\text { Storage } \\
\text { records } \\
\text { pub- } \\
\text { 11shed }\end{array}$} \\
\hline & $\begin{array}{c}\text { Basin } \\
\text { 1ndex and } \\
\text { station no }\end{array}$ & $\begin{array}{l}\text { Lat1tude } \\
\text { and } \\
\text { long1tude }\end{array}$ & & & Dead & Total & Usable & & & & & \\
\hline IDAHO--Cont1nued & & & & & & & & & & & & \\
\hline $\begin{array}{l}\text { Pleasant Valley } \\
\text { Blacks Creek }\end{array}$ & $-13 D D$ & $\begin{array}{rr}43 & 27 \\
116 & 09\end{array}$ & - & 403 & - & 7,900 & - & - & FIM & 1925 & $\begin{array}{l}\text { Pleasant Valley } \\
\text { Irrigation Co }\end{array}$ & - \\
\hline $\begin{array}{l}\text { Portneuf-Marsh Valley } \\
\text { Portneuf River }\end{array}$ & $-13 \mathrm{EM}$ & $\begin{array}{rr}42 & 53 \\
111 & 56\end{array}$ & - & 1,593 & - & 23,695 & - & - & I & 1950 & $\begin{array}{l}\text { Portneuf-Marsh } \\
\text { Valley Canal Co }\end{array}$ & - \\
\hline $\begin{array}{l}\text { Priest Lake } \\
\text { Priest River }\end{array}$ & $12-393000$ & $\begin{array}{rr}48 & 30 \\
216 & 53\end{array}$ & 572 & 23,400 & - & - & a 82,000 & 01 & NPR & 1951 & State of Idaho & $\mathrm{PA}$ \\
\hline $\begin{array}{l}\text { Reservoir A } \\
\text { Sweet Water-Web Creek }\end{array}$ & $-13 \mathrm{BD}$ & $\begin{array}{rr}46 & 23 \\
116 & 51\end{array}$ & - & - & of & 6,682 & 6,682 & - & IM & - & Lewiston Orchards & - \\
\hline $\begin{array}{l}\text { Salmon River Canal Company } \\
\text { Salmon Falls Creek }\end{array}$ & $13-106500$ & $\begin{array}{rr}42 & 13 \\
114 & 44\end{array}$ & al, 610 & 3,400 & 48,000 & 230,650 & 182,650 & 19 & I & 1911 & $\underset{\text { Co }}{\text { Salmon River Canal }}$ & PA \\
\hline $\begin{array}{l}\text { Soda } \\
\text { Bear River }\end{array}$ & $\begin{array}{r}10 \mathrm{HC} \\
10-0794 \quad 00\end{array}$ & $\begin{array}{rr}42 & 38 \\
111 & 42\end{array}$ & 3,840 & 1,070 & - & - & 11,800 & $<05$ & IPR & 1924 & $\begin{array}{l}\text { Utah Power and } \\
\text { L1ght Co }\end{array}$ & - \\
\hline $\begin{array}{l}\text { Twin Lakes (Lola Creek) } \\
\text { Lola Creek }\end{array}$ & $12-419200$ & $\begin{array}{rr}47 & 52 \\
116 & 52\end{array}$ & 412 & 4,040 & - & 31,240 & - & - & I & 1909 & $\begin{array}{l}\text { Tw1n Lakes Reser- } \\
\text { vo1r and Irri- } \\
\text { gation Co, Itd }\end{array}$ & PA \\
\hline $\begin{array}{l}\text { Twin Lakes No }{ }^{1} \\
\text { Mink Creek } c /\end{array}$ & $\begin{array}{r}10 \mathrm{HC} \\
10-088200\end{array}$ & $\begin{array}{rr}42 & 11 \\
111 & 58\end{array}$ & - & 508 & 555 & 14,000 & 13,440 & - & I & 1920 & $\underset{\text { Co }}{\text { Twin Lakes Canal }}$ & - \\
\hline $\begin{array}{l}\text { Wilson Lake } \\
\text { Snake River } \mathrm{c} \\
\text { ILLINOIS }\end{array}$ & $-13 E R$ & $\begin{array}{rr}42 & 37 \\
114 & 10\end{array}$ & - & 1,432 & - & - & 18,500 & - & I & 1909 & $\underset{\text { Corth S1de Canal }}{\text { No }}$ & - \\
\hline $\begin{array}{l}\text { Crab Orchard Lake } \\
\text { Crab Orchard Creek }\end{array}$ & $05-5980$ OMG & $\begin{array}{ll}37 & 43 \\
89 & 09\end{array}$ & 215 & 6,910 & 67,320 & 109,800 & 42,500 & 4 & $\mathrm{CMR}$ & 1939 & $\begin{array}{l}\text { U } \mathrm{S} F 1 \mathrm{sh} \text { and W1ld- } \\
\text { life Service }\end{array}$ & - \\
\hline $\begin{array}{l}\text { Devils Kitchen Lake } \\
\text { Grassy Creek }\end{array}$ & $05-5979 \begin{array}{r}5 \mathrm{MG} \\
30\end{array}$ & $\begin{array}{ll}37 & 38 \\
89 & 06\end{array}$ & 183 & 810 & - & 29,200 & - & - & RW & 1959 & U $\underset{\text { SIfe Service }}{\text { Fish and Wild- }}$ & - \\
\hline $\begin{array}{l}\text { Farmdale } \\
\text { Farm Creek }\end{array}$ & $05-560450$ & $\begin{array}{ll}40 & 40 \\
89 & 30\end{array}$ & 265 & 385 & 0 & 11,300 & 11,300 & - & $\mathrm{F}$ & 1950 & Corps of Englneers & - \\
\hline $\begin{array}{l}\text { Lake Bloomington } \\
\text { Money Creek }\end{array}$ & $05-565450$ & $\begin{array}{ll}40 & 40 \\
88 & 56\end{array}$ & 61 & 487 & 0 & 8,912 & 8,912 & 3 & MR & 1930 & C1ty of Bloomington & $\mathrm{PA}$ \\
\hline $\begin{array}{l}\text { Lake Cooper (Keokuk lock and dam 19)* } \\
\text { M1ss1ss1ppi R1ver }\end{array}$ & $\left|\begin{array}{r}5 \mathrm{HA} \\
05-474500\end{array}\right|$ & $\begin{array}{ll}40 & 24 \\
91 & 22\end{array} \mid$ & 119,000 & 28,200 & - & 337,000 & 26,000 & $<05$ & NPR & 1913 & $\begin{array}{l}\text { Union Electric } \\
\text { Power Co }\end{array}$ & - \\
\hline
\end{tabular}


Lake Decatur
Sangamon R1ver

Lake of Egypt

Saline River

Lake Mattoon

L1ttle Wabash River

Lake Sara

Point Creek

Lake Springfield

Sugar creek

Lake Vermilion
North Fork Vermilion River

Little Grassy Lake

Little Grassy Creek

Omega Lake
Lost Fork

Silver Lake

S1lver Lake
East Fork S1lver Creek

Wonder Lake

Nippersink Creek

\section{INDIANA}

Bean Blossom (Lake Lemon Dam) Bean Blossom Creek

Cagles Mill (Cathact Lake)

Ge1st

Fall Creek

Lake Freeman

TIppecanoe River

Lake Greenwood
First Creek

ake Shafer

Lake Shafer
T1ppecanoe River

ansfield

Raccoon Creek

Morse

Creek

See footnotes at end of table

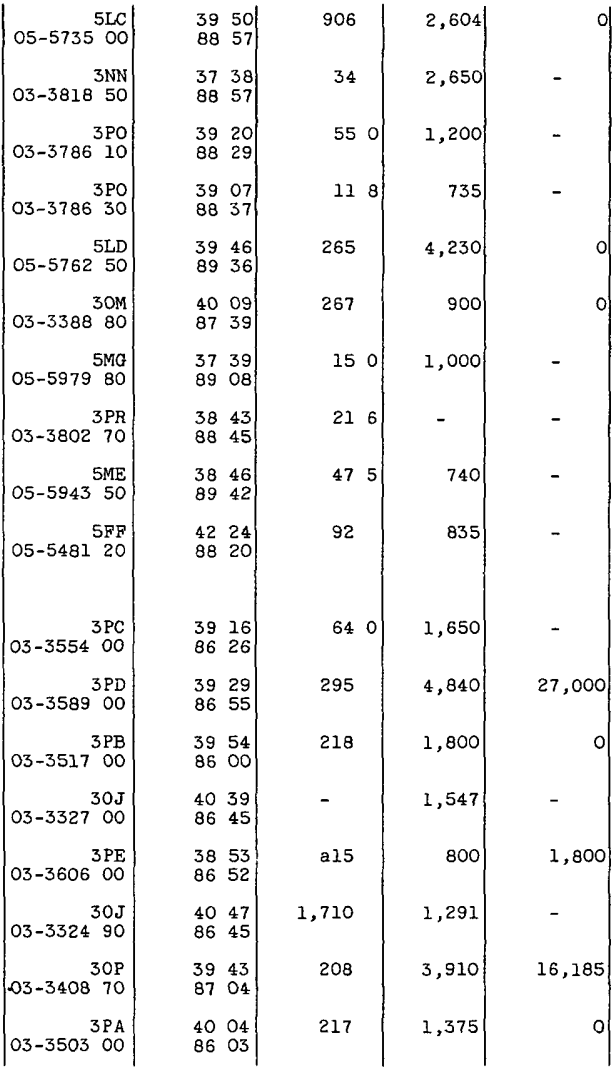

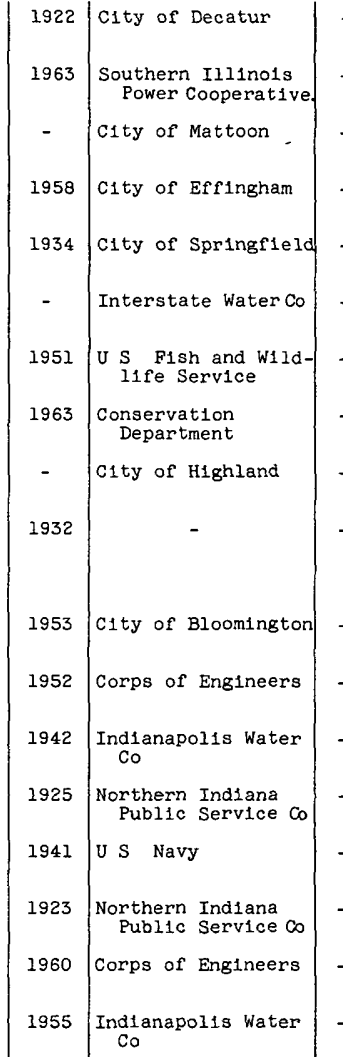


Table 1 --Reservoirs in the Un1ted States completed or under construction as of January 1, 1963, having a usable capac1ty of 5,000 acre-feet or more--Continued

\begin{tabular}{|c|c|c|c|c|c|c|c|c|c|c|c|c|}
\hline \multirow[b]{2}{*}{$\begin{array}{c}\text { Name of reservo1r } \\
\text { and } \\
\text { stream }\end{array}$} & \multicolumn{2}{|c|}{ Location } & \multirow{2}{*}{$\begin{array}{l}\text { Drainage } \\
\text { area } \\
\text { (square } \\
\text { miles) }\end{array}$} & \multirow[b]{2}{*}{$\begin{array}{c}\text { Surface } \\
\text { area } \\
\text { (acres) }\end{array}$} & \multicolumn{3}{|c|}{ Storage in acre-feet } & \multirow[b]{2}{*}{$\mid \begin{array}{c}\text { Storage } \\
\text { rat10 }\end{array}$} & \multirow[b]{2}{*}{ Use } & \multirow[b]{2}{*}{$\begin{array}{c}\text { Date } \\
\text { com- } \\
\text { pleted }\end{array}$} & \multirow[b]{2}{*}{$\begin{array}{l}\text { Owner } \\
\text { or } \\
\text { operator }\end{array}$} & \multirow{2}{*}{$\begin{array}{l}\text { Storage } \\
\text { records } \\
\text { pub- } \\
\text { lished }\end{array}$} \\
\hline & \begin{tabular}{|c|} 
Basin \\
1ndex and \\
station no
\end{tabular} & $\begin{array}{l}\text { Lat1tude } \\
\text { and } \\
\text { longitude }\end{array}$ & & & Dead & Total & Usable & & & & & \\
\hline \multicolumn{13}{|l|}{ INDIANA- Cont Inued } \\
\hline $\begin{array}{l}\text { Prairie Creek } \\
\text { Prairle Creek } \\
\text { IOWA }\end{array}$ & $\begin{array}{r}3 P A \\
03-3469\end{array}$ & $\begin{array}{ll}40 & 09 \\
86 & 17\end{array}$ & 17 & 1,275 & 706 & 22,000 & 21,300 & - & M & 1960 & $\underset{\text { Co }}{\text { Muncle Waterworks }}$ & - \\
\hline $\begin{array}{l}\text { Coralville } \\
\text { Iowa River }\end{array}$ & $\begin{array}{r}5 \mathrm{GC} \\
05-4535 \\
10\end{array}$ & $\begin{array}{ll}41 & 43 \\
91 & 32\end{array}$ & 3,115 & 24,800 & 16,900 & 488,900 & 472,000 & 04 & FR & 1957 & Corps of Engineers & $\mathrm{PA}$ \\
\hline $\begin{array}{l}\text { Lake Cooper (Keokuk lock and dam } \\
\text { No 19)* } \\
\text { Miss1ss1pp1 River }\end{array}$ & $\begin{array}{r}5 \mathrm{HA} \\
05-474500\end{array}$ & $\begin{array}{ll}40 & 24 \\
91 & 22\end{array} \mid$ & 119,000 & 28,200 & - & 337,000 & 26,000 & $<05$ & NPR & 1913 & $\begin{array}{l}\text { Union Electric } \\
\text { Power Co }\end{array}$ & - \\
\hline $\begin{array}{l}\text { MacBride } \\
\text { Mill Creek }\end{array}$ & $05-4535$ $5 \mathrm{GC}$ & $\begin{array}{ll}41 & 48 \\
91 & 34\end{array}$ & 270 & 960 & 0 & 14,400 & 14,400 & - & $\mathrm{R}$ & 1957 & $\begin{array}{c}\text { Iowa State Conser- } \\
\text { vation Comm1ssion }\end{array}$ & PA \\
\hline \multicolumn{13}{|l|}{$\underline{\text { KANSAS }}$} \\
\hline $\begin{array}{l}\text { Cedar Bluff } \\
\text { Smokey Hill River }\end{array}$ & $06-861500$ & $\begin{array}{ll}38 & 47 \\
99 & 43\end{array}$ & 5,530 & 10,790 & 8,261 & 376,950 & 368,690 & - & FIR & 1951 & $\begin{array}{l}\text { Bureau of } \\
\text { Reclamation }\end{array}$ & $\mathrm{PA}$ \\
\hline $\begin{array}{l}\text { Cheyenne Bottoms Wildiffe Refuge } \\
\text { Arkansas and Walnut Rivers } \subseteq\end{array}$ & $\begin{array}{r}7 \mathrm{DB} \\
07-1430 \quad 00\end{array}$ & $\begin{array}{ll}38 & 28 \\
98 & 40\end{array}$ & - & 12,290 & - & 24,080 & - & - & R & 1955 & $\begin{array}{l}\text { Kansas Forestry, } \\
\text { Fish, and Game } \\
\text { Commission }\end{array}$ & - \\
\hline $\begin{array}{l}\text { Clark County State Lake } \\
\text { Bluff Creek }\end{array}$ & $\begin{array}{r}7 \mathrm{DA} \\
07-157750\end{array}$ & $\begin{array}{ll}37 & 23 \\
99 & 47\end{array}$ & 1434 & 337 & - & 7,660 & - & - & $\mathrm{R}$ & 1941 & $\begin{array}{l}\text { Kansas Forestry, } \\
\text { Fish, and Game } \\
\text { Commission }\end{array}$ & - \\
\hline $\begin{array}{l}\text { Council Grove Lake } \\
\text { Canning Creek }\end{array}$ & $07-1793 \begin{array}{r}7 \mathrm{EC} \\
90\end{array}$ & $\begin{array}{ll}38 & 41 \\
96 & 33\end{array}$ & 122 & 387 & - & 8,420 & - & - & $M R$ & 1942 & $\begin{array}{l}\text { City of council } \\
\text { Grove }\end{array}$ & - \\
\hline $\begin{array}{l}\text { Emporia Municipal (Lake Kahola) } \\
\text { Kahola Creek }\end{array}$ & $07-1797 \begin{array}{r}7 \mathrm{EC} \\
10\end{array}$ & $\begin{array}{ll}38 & 31 \\
96 & 25\end{array}$ & 161 & 405 & - & 6,434 & - & - & MR & 1936 & C1ty of Emporla & - \\
\hline $\begin{array}{l}\text { Fall River } \\
\text { Fall RIver }\end{array}$ & $07-1680 \quad 00$ & $\begin{array}{ll}37 & 39 \\
96 & 04\end{array}$ & 585 & 10,400 & 80 & 263,000 & 262,900 & 11 & $\mathrm{CF}$ & 1949 & Corps of Engineers & $\mathrm{PA}$ \\
\hline $\begin{array}{l}\text { Fort Scott } \\
\text { Rock Creek }\end{array}$ & $\begin{array}{r}6 S F \\
06-9174 \\
0\end{array}$ & $\begin{array}{ll}37 & 47 \\
94 & 46\end{array}$ & 117 & 352 & - & 6,485 & - & - & MR & 1958 & C1ty of Fort Scott & - \\
\hline $\begin{array}{l}\text { Kanopolis } \\
\text { Smoky Hill River }\end{array}$ & \begin{tabular}{rr|}
$6 \mathrm{RC}$ \\
$06-8650$ & 00
\end{tabular} & $\begin{array}{ll}38 & 37 \\
97 & 58\end{array}$ & 7,857 & 13,900 & 60 & 432,880 & 432,820 & 16 & $\mathrm{CFR}$ & 1948 & Corps of Engineers & PA \\
\hline $\begin{array}{l}\text { Kirwin } \\
\text { North Fork Solomon River }\end{array}$ & $\left|\begin{array}{rr}6 R F \\
06-8717 & 00\end{array}\right|$ & $\begin{array}{ll}39 & 40 \\
99 & 07\end{array}$ & 1,360 & 10,640 & 6,385 & 314,550 & 308,165 & - & $F I R$ & 1955 & $\begin{array}{l}\text { Bureau of } \\
\text { Reclamation }\end{array}$ & - \\
\hline
\end{tabular}


Lake Bluestem
Harrison Creek

Lake McKinney

Arkansas River c/

Lovewell
White Rock Creek and diversion

Lowell (RIverton)

Spring River and Shoal Creek

Parsons

Labette Creek

Shawnee Lake

Toronto

Verdigr1s River

Tuttle Creek

Webster

South Fork Solomon River

Wyandotte County Lake

Marshall Creek

\section{KENTUCKY}

Buckhorn
M1ddle Fork Kentucky River

Dewey

Herrington Lake (D1x Dam)

Kentucky Lake
Tennessee R1ver

Lake Cumberland (Wolf Creek Dam)

Rough River
Rough River

\section{LOUISIANA}

Anacoco Lake
Bayou Anacoco

See footnotes at end of table

\begin{tabular}{|c|c|c|c|c|}
\hline $\begin{array}{r}7 D C \\
07-1466 \quad 20\end{array}$ & $\begin{array}{ll}37 & 51 \\
96 & 47\end{array}$ & 486 & 845 & - \\
\hline $\begin{array}{r}7 \mathrm{DA} \\
07-1385 \quad 10\end{array}$ & $\begin{array}{rr}37 & 59 \\
101 & 12\end{array}$ & - & 3,000 & - \\
\hline $\begin{array}{r}6 R P \\
06-8539 \stackrel{00}{ }\end{array}$ & $\begin{array}{ll}39 & 54 \\
98 & 02\end{array}$ & 342 & 5,025 & 5,050 \\
\hline $07-1875 \stackrel{7 \mathrm{ED}}{80}$ & $\begin{array}{ll}94 & 43 \\
37 & 04\end{array}$ & 2,210 & 862 & - \\
\hline $07-1844 \stackrel{7 \mathrm{ED}}{80}$ & $\begin{array}{ll}37 & 24 \\
95 & 20\end{array}$ & 375 & 980 & - \\
\hline $\begin{array}{r}6 R T \\
06-889600\end{array}$ & $\begin{array}{ll}39 & 01 \\
95 & 38\end{array}$ & $\begin{array}{ll}9 & 7\end{array}$ & 411 & - \\
\hline $\begin{array}{r}7 \mathrm{~EB} \\
07-1659 \quad 00\end{array}$ & $\begin{array}{ll}37 & 44 \\
95 & 56\end{array}$ & 730 & 10,300 & 13,000 \\
\hline $\begin{array}{r}6 R S \\
06-886900\end{array}$ & $\begin{array}{ll}39 & 15 \\
96 & 36\end{array}$ & 9,560 & 54,050 & 233,000 \\
\hline $\begin{array}{r}6 R E \\
06-873100\end{array}$ & $\begin{array}{ll}39 & 25 \\
99 & 25\end{array}$ & 1,200 & 8,480 & 2,180 \\
\hline $\begin{array}{r}6 \mathrm{PE} \\
06-8212 \quad 55\end{array}$ & $\begin{array}{ll}38 & 10 \\
94 & 46\end{array}$ & 80 & 305 & - \\
\hline $03-2808^{3 L C}$ & $\begin{array}{ll}37 & 20 \\
83 & 28\end{array}$ & 408 & 3,610 & 10,280 \\
\hline $03-2110$ 3GL & $\begin{array}{ll}37 & 44 \\
82 & 44\end{array}$ & 206 & 3,340 & 12,300 \\
\hline $\begin{array}{r}3 L G \\
03-2860 \quad 00\end{array}$ & $\begin{array}{ll}37 & 47 \\
84 & 42\end{array}$ & 439 & 3,600 & - \\
\hline $03-6090$ WJ & $\begin{array}{ll}37 & 01 \\
88 & 16\end{array}$ & 40,200 & 261,000 & $1,991,800$ \\
\hline $\begin{array}{r}3 R F \\
03-4135 \stackrel{00}{ }\end{array}$ & $\begin{array}{ll}36 & 52 \\
85 & 09\end{array}$ & 5,789 & 63,530 & $1,853,000$ \\
\hline $\begin{array}{r}\text { 3NG } \\
03-3180 \text { 05 }\end{array}$ & $\begin{array}{ll}37 & 37 \\
86 & 30\end{array}$ & 454 & 10,260 & 20,170 \\
\hline $\begin{array}{r}80 \mathrm{~A} \\
08-0277^{10}\end{array}$ & $\begin{array}{ll}31 & 06 \\
93 & 23\end{array}$ & 213 & 2,600 & 0 \\
\hline
\end{tabular}

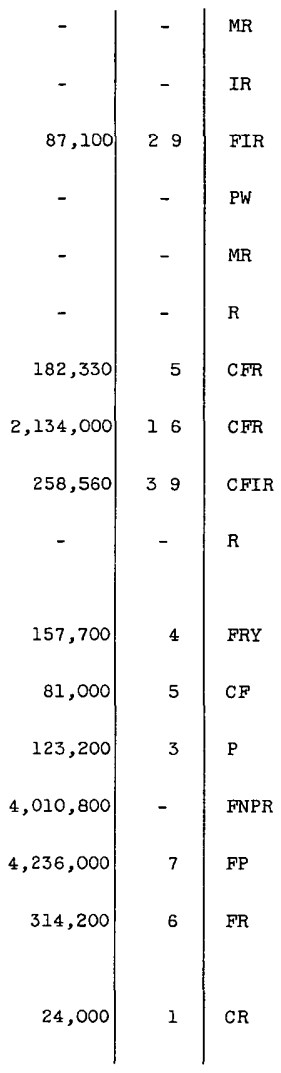


Table 1 --Reservoirs in the United States completed or under construction as of January 1, 1963, having a usable capacity of 5,000 acre-feet or more--Continued [For meaning of symbols under "Use" and "Storage records published," see text]

\begin{tabular}{|c|c|c|c|c|c|c|c|c|c|c|c|c|}
\hline \multirow[b]{2}{*}{$\begin{array}{l}\text { Name of reservo1r } \\
\text { and } \\
\text { stream }\end{array}$} & \multicolumn{2}{|c|}{ Location } & \multirow{2}{*}{$\begin{array}{l}\text { Drainage } \\
\text { area } \\
\text { (square } \\
\text { m1les) }\end{array}$} & \multirow[b]{2}{*}{$\begin{array}{c}\text { Surface } \\
\text { area } \\
\text { (acres) }\end{array}$} & \multicolumn{3}{|c|}{ Storage in acre-feet } & \multirow[b]{2}{*}{$\begin{array}{r}\text { Storage } \\
\text { rat10 }\end{array}$} & \multirow[b]{2}{*}{ Use } & \multirow[b]{2}{*}{$\left|\begin{array}{c}\text { Date } \\
\text { com- } \\
\text { pleted }\end{array}\right|$} & \multirow[b]{2}{*}{$\begin{array}{l}\text { Owner } \\
\text { or } \\
\text { operator }\end{array}$} & \multirow{2}{*}{$\begin{array}{l}\text { Storage } \\
\text { records } \\
\text { pub- } \\
\text { lished }\end{array}$} \\
\hline & \begin{tabular}{|c|} 
Basin \\
1ndex and \\
station no
\end{tabular} & $\begin{array}{c}\text { Lat1 tude } \\
\text { and } \\
\text { long1 tude }\end{array}$ & & & Dead & Total & Usable & & & & & \\
\hline LOUIS IANA Continued & & & & & & & & & & & & \\
\hline $\begin{array}{l}\text { Bayou DeS1ard } \\
\text { Bayou DeStard }\end{array}$ & $\begin{array}{r}70 \mathrm{~A} \\
07-3665 \quad 00\end{array}$ & $\begin{array}{ll}32 & 33 \\
92 & 07\end{array}$ & 32 & 1,100 & - & 13,520 & - & - & IMR & 1928 & $\begin{array}{c}\text { C1ty of Monroe and } \\
\text { Ouachita Par1sh }\end{array}$ & $\mathrm{PA}$ ef \\
\hline $\begin{array}{l}\text { Biack Lake } \\
\text { Black Bayou }\end{array}$ & $07-346490$ & $\begin{array}{ll}32 & 53 \\
93 & 54\end{array}$ & 231 & 3,960 & 0 & 17,750 & 17,750 & 01 & $\mathrm{CR}$ & 1945 & $\begin{array}{l}\text { Black Bayou F1sh } \\
\text { and Game Comm }\end{array}$ & - \\
\hline $\begin{array}{l}\text { Black Lake-Clear Lake } \\
\text { Black Lake Bayou }\end{array}$ & 07-3528 90 & $\begin{array}{ll}31 & 51 \\
92 & 58\end{array} \mid$ & 920 & 13,800 & o & 109,000 & 109,000 & 2 & $C R$ & 1934 & $\begin{array}{l}\text { Northwest Louisiana } \\
\text { Game and Fish } \\
\text { Commission }\end{array}$ & - \\
\hline $\begin{array}{l}\text { Bodcau Bayou } \\
\text { Bodcau Bayou }\end{array}$ & $\begin{array}{r}7 M A \\
07-349650\end{array}$ & $\begin{array}{ll}32 & 42 \\
93 & 31\end{array}$ & 683 & 44,950 & o & 967,900 & - & - & $F$ & 1949 & Corps of Engineers & - \\
\hline $\begin{array}{l}\text { Bundick Lake } \\
\text { Bundick Creek }\end{array}$ & $08-014880$ & $\begin{array}{ll}30 & 43 \\
93 & 06\end{array}$ & 208 & 1,750 & 0 & 9,200 & 9,200 & $<05$ & $R$ & 1961 & $\begin{array}{c}\text { Bund1ck Creek Game } \\
\text { and F1sh Preserve }\end{array}$ & - \\
\hline $\begin{array}{l}\text { Caddo Lake } \\
\text { Cypress Creek }\end{array}$ & $07-3463 \quad 10$ & $\begin{array}{ll}32 & 42 \\
93 & 55\end{array}$ & 2,744 & 32,500 & 187,500 & 187,500 & 0 & - & $\mathrm{R}$ & 1914 & Corps of Eng1neers & - \\
\hline $\begin{array}{l}\text { Cane River Lake } \\
\text { Cane RIver }\end{array}$ & $07-3547 \quad 7 \mathrm{MA}$ & $\begin{array}{ll}31 & 34 \\
92 & 58\end{array} \mid$ & 34 & 1,350 & - & a.2,, 000 & - & - & $\mathrm{R}$ & 1949 & $\begin{array}{l}\text { Natch1 toches Parish } \\
\text { Pollce Jury }\end{array}$ & - \\
\hline $\begin{array}{l}\text { Chenlere Brake } \\
\text { Chenfere Creek }\end{array}$ & $\begin{array}{r}70 c \\
07-367500\end{array}$ & $\begin{array}{ll}32 & 27 \\
92 & 12\end{array}$ & 140 & 3,400 & 2,500 & 24,000 & 21,500 & 2 & $\mathrm{CR}$ & 1944 & $\begin{array}{c}\text { Cheniere Brake F1sh } \\
\text { Commission }\end{array}$ & - \\
\hline $\begin{array}{l}\text { Clear Lake-Smi thport Lake } \\
\text { Rambin Bayou }\end{array}$ & $07-3517 \begin{array}{r}7 \mathrm{MA} \\
0\end{array}$ & $\begin{array}{ll}32 & 07 \\
93 & 34\end{array}$ & 205 & 2,950 & 1,800 & 11,500 & 9,700 & 06 & $\mathrm{CR}$ & 1948 & $\begin{array}{l}\text { Bayou P1erre Game } \\
\text { and F1sh Comm }\end{array}$ & - \\
\hline $\begin{array}{l}\text { Cocodrie Lake } \\
\text { Bayou Cocodrie }\end{array}$ & $07-3819 \quad 50$ & $\begin{array}{ll}31 & 00 \\
92 & 23\end{array}$ & 240 & 6,100 & 0 & 11,000 & 11,000 & $<05$ & $R$ & 1959 & $\begin{array}{l}\text { Rapids and Evange- } \\
\text { line Partsh } \\
\text { Police Juries }\end{array}$ & - \\
\hline $\begin{array}{l}\text { Corney Lake } \\
\text { Corney Bayou }\end{array}$ & $\begin{array}{r}70 B \\
07-365970\end{array}$ & $\begin{array}{ll}32 & 54 \\
92 & 44\end{array}$ & 2400 & 1,900 & 2,000 & 8,000 & 6,000 & $<05$ & $\mathrm{CR}_{\mathrm{R}}$ & 1940 & $\begin{array}{l}\text { Corney Lake Game } \\
\text { and Fish Comm }\end{array}$ & - \\
\hline $\begin{array}{l}\text { Cross Lake (Shreveport) } \\
\text { Cross Bayou }\end{array}$ & $07-348488$ & $\begin{array}{ll}32 & 31 \\
93 & 48\end{array}$ & 256 & 8,840 & 28,000 & 77,600 & 49,600 & 3 & MR & 1925 & C1ty of Shreveport & - \\
\hline $\begin{array}{l}\text { Iatt Lake } \\
\text { Iatt Creek }\end{array}$ & $07-3553 \quad 00$ & $\begin{array}{ll}31 & 33 \\
92 & 39\end{array}$ & 242 & 5,050 & of & 13,500 & 13,500 & 06 & $\mathrm{R}$ & 1957 & $\begin{array}{l}\text { Iatt Lake Game and } \\
\text { Fish Commission }\end{array}$ & - \\
\hline $\begin{array}{l}\text { Kepler Lake } \\
\text { Kepler Creek }\end{array}$ & $07-352410$ & $\begin{array}{ll}32 & 19 \\
93 & 10\end{array}$ & 462 & 1,925 & 900 & 16,800 & 15,900 & 4 & R & 1958 & $\begin{array}{l}\text { B1enville Parish } \\
\text { Pollce Jury }\end{array}$ & - \\
\hline $\begin{array}{l}\text { Lake Bistineau } \\
\text { Bayou Dorcheat }\end{array}$ & $|07-349250|$ & $\begin{array}{ll}32 & 20 \\
93 & 26\end{array}$ & 1,458 & 17,200 & 36,000 & 120,400 & 84,400 & 07 & $\mathrm{CR}$ & 1942 & $\left|\begin{array}{c}\text { Lake Bistineau Game } \\
\text { and F1sh Comm }\end{array}\right|$ & - \\
\hline
\end{tabular}




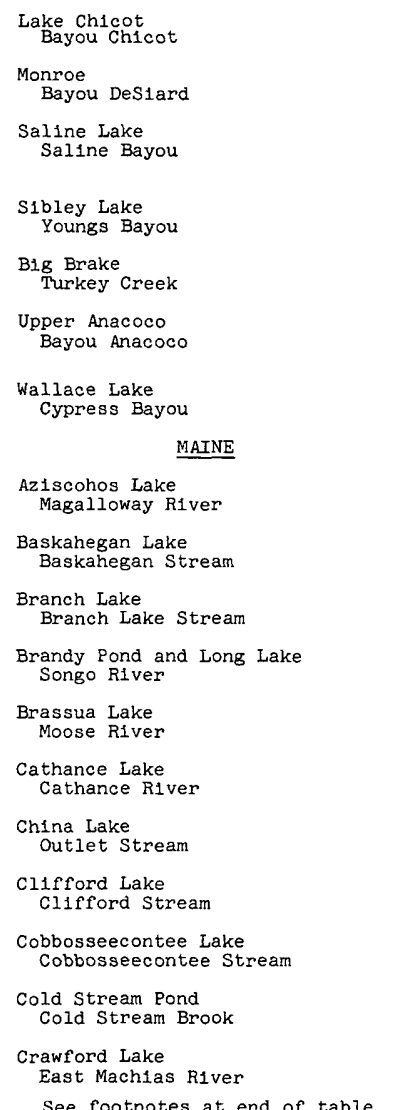

\begin{tabular}{|c|c|c|c|c|c|}
\hline $\begin{array}{rr}7 Q D \\
07-3821 \\
23\end{array}$ & $\begin{array}{ll}30 & 49 \\
92 & 16\end{array}$ & 57 of & 1,625 & 220 & 9,700 \\
\hline $07-3665 \quad 00$ & $\begin{array}{ll}32 & 33 \\
92 & 07\end{array}$ & - & - & - & 6,500 \\
\hline $\begin{array}{r}7 \mathrm{MA} \\
07-3521 \\
50\end{array}$ & $\begin{array}{ll}31 & 52 \\
92 & 56\end{array}$ & 420 & 8,950 & 6,500 & 61,000 \\
\hline $\begin{array}{rr}7 \mathrm{MA} \\
07-3537 & 90\end{array}$ & $\begin{array}{ll}31 & 45 \\
93 & 07\end{array}$ & 40 & 2,175 & 1,200 & 19,500 \\
\hline $\begin{array}{r}70 C \\
07-3692 \\
70\end{array} \mid$ & $\begin{array}{ll}31 & 54 \\
91 & 46\end{array}$ & 163 & 3,100 & 2,100 & 20,500 \\
\hline $\begin{array}{r}80 \mathrm{~A} \\
08-0274 \quad 50\end{array}$ & $\begin{array}{ll}31 & 11 \\
93 & 22\end{array}$ & 116 & 4,300 & d & 58,000 \\
\hline $\begin{array}{r}7 \mathrm{7MA} \\
07-3515 \quad 50\end{array}$ & $\begin{array}{ll}32 & 19 \\
93 & 40\end{array}$ & 266 & 9,250 & $b 7,760$ & 96,100 \\
\hline $\begin{array}{r}1 \mathrm{DA} \\
01-0520 \quad 00\end{array}$ & $\begin{array}{ll}44 & 57 \\
71 & 00\end{array} \mid$ & 214 & 7,100 & - & - \\
\hline $01-0302^{1 B G}$ & $\begin{array}{ll}45 & 32 \\
67 & 50\end{array}$ & 154 & 10,496 & - & - \\
\hline $\begin{array}{r}1 \mathrm{IBC} \\
01-0255 \quad 00\end{array}$ & $\begin{array}{ll}44 & 34 \\
68 & 30\end{array}$ & 31 & 2,770 & - & - \\
\hline $\begin{array}{rr}1 D D \\
01-0630 & 00\end{array} \mid$ & $\begin{array}{ll}43 & 56 \\
70 & 35\end{array}$ & 120 & - & - & - \\
\hline $\begin{array}{r}1 \mathrm{CB} \\
01-0385^{2} 00\end{array}$ & $\begin{array}{ll}45 & 40 \\
69 & 49\end{array}$ & 715 & 9,660 & 0 & 196,500 \\
\hline \begin{tabular}{rr|r}
$1 B B$ \\
$01-0211$ & 00
\end{tabular} & $\begin{array}{ll}44 & 56 \\
67 & 24\end{array}$ & 24 & 3,437 & - & - \\
\hline $\begin{array}{r}I C E \\
01-049200\end{array}$ & $\begin{array}{ll}44 & 27 \\
69 & 36\end{array}$ & 36 & 3,853 & - & - \\
\hline $\begin{array}{rr}1 \mathrm{BA} \\
01-0191 & 00\end{array}$ & $\begin{array}{ll}45 & 04 \\
67 & 42\end{array}$ & 14 & 1,430 & o & 16,700 \\
\hline $01-0494 \stackrel{1 C F}{00}$ & $\begin{array}{ll}44 & 17 \\
69 & 53\end{array}$ & 133 & 5,440 & - & - \\
\hline $\begin{array}{rr}1 \mathrm{BH} \\
01-0354 & 00\end{array}$ & $\begin{array}{ll}45 & 15 \\
68 & 34\end{array}$ & 28 & 4,723 & - & - \\
\hline $\begin{array}{rr}1 \mathrm{BB} \\
01-0219 & 00\end{array} \mid$ & $\begin{array}{ll}45 & 01 \\
67 & 36\end{array} \mid$ & 60 & 1,715 & - & - \\
\hline
\end{tabular}

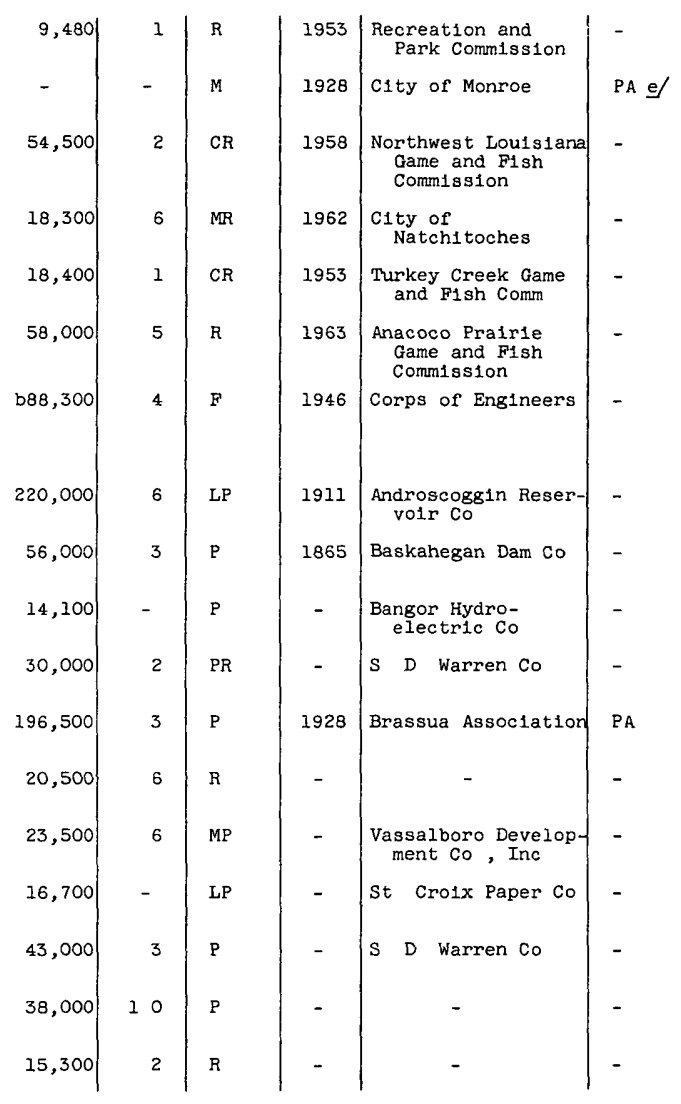




\begin{tabular}{|c|c|c|c|c|c|c|c|c|c|c|c|c|}
\hline \multirow[b]{2}{*}{$\begin{array}{l}\text { Name of reservoir } \\
\text { and } \\
\text { stream }\end{array}$} & \multicolumn{2}{|c|}{ Location } & \multirow{2}{*}{$\begin{array}{l}\text { Drainage } \\
\text { area } \\
\text { (square } \\
\text { miles) }\end{array}$} & \multirow[b]{2}{*}{$\begin{array}{c}\text { Surface } \\
\text { area } \\
\text { (acres) }\end{array}$} & \multicolumn{3}{|c|}{ Storage in acre-feet } & \multirow[b]{2}{*}{$\begin{array}{c}\text { Storage } \\
\text { rat10 }\end{array}$} & \multirow[b]{2}{*}{ Use } & \multirow[b]{2}{*}{$\begin{array}{c}\text { Date } \\
\text { com- } \\
\text { pleted }\end{array}$} & \multirow[b]{2}{*}{$\begin{array}{l}\text { Owner } \\
\text { or } \\
\text { operator }\end{array}$} & \multirow{2}{*}{$\begin{array}{l}\text { Storage } \\
\text { records } \\
\text { pub- } \\
\text { lished }\end{array}$} \\
\hline & \begin{tabular}{|c|} 
Basin \\
1ndex and \\
station no.
\end{tabular} & $\begin{array}{l}\text { Lat1 tude } \\
\text { and } \\
\text { long1 tude }\end{array}$ & & & Dead & Total & Usable & & & & & \\
\hline MAINE--Cont1nued & & & & & & & & & & & & \\
\hline $\begin{array}{l}\text { Crotched Pond } \\
\text { Dead RIver }\end{array}$ & $\begin{array}{r}1 D C \\
01-055200\end{array}$ & $\begin{array}{ll}44 & 25 \\
70 & 02\end{array} \mid$ & 46 & 1,070 & - & - & 10,700 & 02 & $\mathrm{R}$ & - & - & - \\
\hline $\begin{array}{l}\text { Damariscotta Lake } \\
\text { Damariscotta RIver }\end{array}$ & $\mid \begin{array}{r}1 C A \\
01-037900\end{array}$ & $\begin{array}{ll}44 & 04 \\
69 & 32\end{array} \mid$ & 570 & 4,345 & - & - & 13,100 & - & $\mathrm{P}$ & 1921 & $\begin{array}{l}\text { Central Maine } \\
\text { Power co }\end{array}$ & - \\
\hline $\begin{array}{l}\text { Ellis and McGrath Ponds } \\
\text { Tributary to Great Pond }\end{array}$ & $\begin{array}{r}1 \mathrm{CE} \\
01-0492 \quad 20\end{array}$ & $\begin{array}{ll}44 & 31 \\
69 & 48\end{array}$ & 9 & 1,060 & - & - & 6,300 & 6 & $\mathrm{P}$ & 1960 & $\begin{array}{l}\text { Central Maine } \\
\text { Power Co }\end{array}$ & - \\
\hline $\begin{array}{l}\text { Embden Pond } \\
\text { M1ll Stream }\end{array}$ & $\begin{array}{r}1 C D \\
01-047100\end{array}$ & $\begin{array}{ll}44 & 55 \\
69 & 56\end{array}$ & 29 & 1,500 & - & - & 6,100 & 2 & $\mathrm{R}$ & - & $\begin{array}{l}\text { Maine Department of } \\
\text { Inland FIsheries } \\
\text { and Game }\end{array}$ & - \\
\hline $\begin{array}{l}\text { Estes Lake } \\
\text { Mousam River }\end{array}$ & $\mid \begin{array}{r}1 D H \\
01-069000\end{array}$ & $\begin{array}{ll}43 & 26 \\
70 & 40\end{array}$ & 105 & - & - & - & 5,350 & $<05$ & $\mathrm{P}$ & - & - & - \\
\hline $\begin{array}{l}\text { First Roach Pond } \\
\text { Roach River }\end{array}$ & O1-0400 00 & $\begin{array}{ll}45 & 41 \\
69 & 26\end{array}$ & 696 & 3,140 & 0 & 21,500 & 21,500 & 3 & LP & - & Roach River Dam Co & PA \\
\hline $\begin{array}{l}\text { Flagstaff Lake } \\
\text { Dead River }\end{array}$ & $01-0430 \quad 00$ & $\begin{array}{ll}45 & 13 \\
70 & 12\end{array}$ & 520 & - & 0 & 276,000 & 276,000 & 5 & $L P$ & 1950 & $\begin{array}{l}\text { Central Maine Power } \\
\text { Co }\end{array}$ & $\mathrm{PA}$ \\
\hline $\begin{array}{l}\text { Gardner Lake } \\
\text { East Machias River }\end{array}$ & $\mid \begin{array}{r}1 B B \\
01-0221 \quad 00\end{array}$ & $\begin{array}{ll}44 & 45 \\
67 & 20\end{array}$ & 57 & 5,376 & - & - & 27,000 & 3 & $\mathrm{R}$ & - & Town of East Machias & - \\
\hline $\begin{array}{l}\text { Graham Lake } \\
\text { Union River }\end{array}$ & $\begin{array}{r}1 \mathrm{BC} \\
01-0252 \quad 00\end{array}$ & $\begin{array}{ll}44 & 36 \\
68 & 26\end{array}$ & 452 & 12,000 & - & - & 140,000 & - & $\mathrm{P}$ & 1922 & $\begin{array}{l}\text { Bangor Hydro-elec- } \\
\text { tric Co }\end{array}$ & - \\
\hline $\begin{array}{l}\text { Grand Lake (Eastern) and North Lake } \\
\text { St Cro1x River }\end{array}$ & |r-0182 00 & $\begin{array}{ll}45 & 50 \\
67 & 46\end{array}$ & 138 & 16,800 & - & - & 105,000 & 7 & $P$ & - & St Cro1x Paper Co & - \\
\hline $\begin{array}{l}\text { Grand Lake (Matagamon) } \\
\text { East Branch Penobscot River }\end{array}$ & $\begin{array}{r}1 \mathrm{BEE} \\
01-0290 \quad 00\end{array}$ & $\begin{array}{ll}46 & 08 \\
68 & 47\end{array}$ & 470 & 4,130 & 0 & 41,000 & 41,000 & 07 & LP & 1942 & $\begin{array}{l}\text { Bangor Hydro-elec- } \\
\text { tric Co }\end{array}$ & PA \\
\hline $\begin{array}{l}\text { Grand Lake (Western) } \\
\text { Grand Lake Stream }\end{array}$ & $01-0190 \quad 00$ & $\begin{array}{ll}45 & 10 \\
67 & 46\end{array}$ & 224 & 23,825 & - & - & 161,000 & 7 & $P$ & - & St Croix Paper Co & - \\
\hline $\begin{array}{l}\text { Great East Lake * } \\
\text { Salmon Falls River }\end{array}$ & $01-0700 \quad 00$ & $\begin{array}{ll}43 & 35 \\
70 & 56\end{array}$ & 170 & 1,690 & 0 & 11,800 & 11,800 & 9 & $P$ & - & $\begin{array}{c}\text { New Hampshire Water } \\
\text { Resources Board }\end{array}$ & - \\
\hline $\begin{array}{l}\text { Great Falls Flowage } \\
\text { St Cro1x River }\end{array}$ & $01-019900$ & $\begin{array}{ll}45 & 19 \\
67 & 28\end{array}$ & 1,320 & 17,600 & - & - & 88,000 & 06 & $P$ & 1914 & St Croix Paper Co & - \\
\hline $\begin{array}{l}\text { Great Pond } \\
\text { Messalonskee Stream }\end{array}$ & $\begin{array}{r}1 \mathrm{CE} \\
01-049230\end{array}$ & $\begin{array}{ll}44 & 31 \\
69 & 52\end{array}$ & 82 & 8,198 & - & - & 49,000 & 5 & $P$ & 1928 & $\underset{\text { Contral Maine Power }}{\text { Cen }}$ & - \\
\hline
\end{tabular}


Green Lake

Green Lake Stream

Gulf Isiand Pond

Androscoggin River

Highland Lake
Stevens Brook

Ind 1 an Pond

Kennebago Lake

Kennebago Stream

Kezar Lake
Kezar Outle

Kingsbury Pond

Kingsbury Stream

Lake Auburn

Outlet Stream to Andros River

Lake Maranacook

Tributary to Lake Annabessacook

Lake Wassookeag
East Branch Sebasticook River

Littie Sebago Lake

Lower Sysladobsis Lake

Grand Lake Stream

Madawaska Lake

L1ttle Madawaska River

Megunticook Lake

Messalonskee Lake

M1linocket Lake

M11linocket Stream

Millinocket Lake

MiIlinocket Stream

Moose Pond

Moose Pond Brook

Moose Pond (Great Moose Lake) Sabasticook River

See footnotes at end of table

\begin{tabular}{|c|c|c|c|c|c|}
\hline $01-024500$ & $\begin{array}{ll}44 & 38 \\
68 & 27\end{array}$ & 47 & 2,840 & - & - \\
\hline $\begin{array}{r}1 D C \\
01-056000\end{array}$ & $\begin{array}{ll}44 & 09 \\
70 & 12\end{array}$ & 2,860 & - & 31,800 & 57,100 \\
\hline 1DD & $\begin{array}{ll}44 & 03 \\
70 & 43\end{array}$ & 20 & 1,340 & - & - \\
\hline $\begin{array}{r}1 \mathrm{ICB} \\
01-041500\end{array}$ & $\begin{array}{ll}45 & 28 \\
69 & 51\end{array}$ & 1,382 & - & 53,300 & 72,300 \\
\hline $01-0500$ 1DA & $\begin{array}{ll}45 & 06 \\
70 & 47\end{array}$ & 112 & 2,560 & o & 21,100 \\
\hline $01-0645 \quad \begin{array}{r}\text { IDE } \\
70\end{array}$ & $\begin{array}{ll}44 & 08 \\
70 & 57\end{array}$ & $\begin{array}{ll}58 & 5\end{array}$ & 2,510 & - & - \\
\hline $\begin{array}{r}1 \mathrm{BH} \\
01-0314 \quad 00\end{array}$ & $\begin{array}{ll}45 & 07 \\
69 & 39\end{array}$ & - & 580 & - & - \\
\hline $01-056500$ & $\begin{array}{ll}44 & 09 \\
70 & 14\end{array}$ & 176 & 2,221 & - & - \\
\hline $01-0493^{1 C F}$ & $\begin{array}{ll}44 & 18 \\
69 & 58\end{array}$ & 33 & 1,640 & - & - \\
\hline $\begin{array}{r}\text { ICE } \\
01-048600\end{array}$ & $\begin{array}{ll}45 & 02 \\
69 & 19\end{array}$ & - & 1,090 & - & - \\
\hline $01-064100$ & $\begin{array}{ll}43 & 41 \\
70 & 26\end{array}$ & 186 & - & - & - \\
\hline $01-0188^{1 B A} 00$ & $\begin{array}{ll}45 & 12 \\
67 & 58\end{array}$ & 44 & 5,410 & - & - \\
\hline $01-017200$ & $\begin{array}{ll}47 & 00 \\
68 & 12\end{array}$ & 316 & 1,820 & - & - \\
\hline $\begin{array}{r}1 \mathrm{CA} \\
01-0374 \quad 10\end{array}$ & $\begin{array}{ll}44 & 13 \\
69 & 05\end{array}$ & 22 & 1,240 & - & - \\
\hline $01-049250$ & $\begin{array}{ll}44 & 32 \\
69 & 44\end{array} \mid$ & 275 & 3,424 & - & - \\
\hline $01-015500$ & $\begin{array}{ll}46 & 18 \\
68 & 50\end{array}$ & 69 & - & - & - \\
\hline $01-0270 \quad 2 B E$ & $\begin{array}{ll}45 & 44 \\
68 & 44\end{array}$ & 106 & 8,928 & - & - \\
\hline $\begin{array}{r}1 \mathrm{DE} \\
01-0645^{9}\end{array}$ & $\begin{array}{ll}43 & 58 \\
70 & 49\end{array} \mid$ & - & 1,660 & - & - \\
\hline $01-0487$ 1CE & $\begin{array}{ll}44 & 54 \\
69 & 29\end{array}$ & 235 & 3,520 & - & - \\
\hline
\end{tabular}


Table 1 --Reservoirs in the Unlted States completed or under construction as of January 1, 1963, having a usable capac1ty of 5,000 acre-feet or more--Continued

\begin{tabular}{|c|c|c|c|c|c|c|c|c|c|c|c|c|}
\hline \multirow[b]{2}{*}{$\begin{array}{l}\text { Name of reservolr } \\
\text { and } \\
\text { stream }\end{array}$} & \multicolumn{2}{|c|}{ Location } & \multirow{2}{*}{$\begin{array}{l}\text { Dralnage } \\
\text { area } \\
\text { (square } \\
\text { m1les) }\end{array}$} & \multirow[b]{2}{*}{$\begin{array}{c}\text { Surface } \\
\text { area } \\
\text { (acres) }\end{array}$} & \multicolumn{3}{|c|}{ Storage in acre-feet } & \multirow[b]{2}{*}{$\begin{array}{c}\text { Storage } \\
\text { rat10 }\end{array}$} & \multirow[b]{2}{*}{ Use } & \multirow[b]{2}{*}{$\begin{array}{c}\text { Date } \\
\text { com- } \\
\text { pleted }\end{array}$} & \multirow[b]{2}{*}{$\begin{array}{l}\text { Owner } \\
\text { or } \\
\text { operator }\end{array}$} & \multirow{2}{*}{$\begin{array}{c}\text { Storage } \\
\text { records } \\
\text { pub- } \\
\text { I1shed }\end{array}$} \\
\hline & \begin{tabular}{|c|} 
Basin \\
1ndex and \\
station no \\
\end{tabular} & $\begin{array}{l}\text { Lat1 tude } \\
\text { and } \\
\text { long1 tude }\end{array}$ & & & Dead & Total & Usable & & & & & \\
\hline MAINE--Cont1nued & & & & & & & & & & & & \\
\hline $\begin{array}{l}\text { Moosehead Lake } \\
\text { Kennebec R1ver }\end{array}$ & $\begin{array}{r}1 C B \\
01-040500\end{array}$ & $\begin{array}{ll}45 & 35 \\
69 & 43\end{array}$ & 1,266 & 74,200 & 0 & 544,900 & 544,900 & 04 & LP & $1840 \mathrm{a}$ & $\begin{array}{l}\text { Kennebec Log } \\
\text { Driving Associates }\end{array}$ & $\mathrm{PA}$ \\
\hline $\begin{array}{l}\text { Mooselookmeguntic Lake (Upper Dam) } \\
\text { Tr1butary to Rap1d River }\end{array}$ & 01-0510 00 & $\begin{array}{ll}44 & 53 \\
70 & 52\end{array}$ & 405 & 16,600 & o & 192,000 & 192,000 & 4 & LP & 1852 & $\underset{\text { Union water Power }}{\text { Co }}$ & PA \\
\hline $\begin{array}{l}\text { Mousam Lake } \\
\text { Mousam River }\end{array}$ & $01-0685$ DD & $\begin{array}{ll}43 & 30 \\
70 & 51\end{array}$ & 310 & 705 & - & - & 9,450 & 3 & $\mathrm{PR}$ & - & Town of Sanford & PA \\
\hline $\begin{array}{l}\text { Moxie Pond } \\
\text { Mox1e Stream }\end{array}$ & 01-0420 $1 \mathrm{1CB}$ & $\begin{array}{ll}45 & 21 \\
69 & 52\end{array}$ & 817 & 1,740 & 0 & 14,700 & 14,700 & 2 & LP & 1923 & Moxie Associates & PA \\
\hline $\begin{array}{l}\text { North Tw1n Lake } \\
\text { West Branch Penobscot River }\end{array}$ & $01-0270 \quad 00$ & $\begin{array}{ll}45 & 39 \\
68 & 47\end{array}$ & 1,864 & - & - & - & 344,000 & 2 & LP & - & $\begin{array}{l}\text { Great Northern } \\
\text { Paper Co }\end{array}$ & PA \\
\hline $\begin{array}{l}\text { Panther and Rattlesnake Ponds } \\
\text { Panther Run }\end{array}$ & | $01-0605$ 1DD & $\begin{array}{ll}43 & 54 \\
70 & 28\end{array}$ & 30 & 2,210 & - & - & 9,300 & 3 & $\mathrm{R}$ & - & - & - \\
\hline $\begin{array}{l}\text { Parmachenee Lake } \\
\text { Magalloway River }\end{array}$ & $01-0519$ IDA & $\begin{array}{ll}45 & 08 \\
70 & 58\end{array}$ & 105 & 960 & - & - & 10,200 & 06 & $\mathrm{~L}$ & - & Brown Co & - \\
\hline $\begin{array}{l}\text { Pleasant Lake and Parker Pond } \\
\text { Mile Brook }\end{array}$ & $01-061500$ & $\begin{array}{ll}44 & 01 \\
70 & 32\end{array}$ & 101 & 1,230 & - & - & 6,150 & 6 & $\mathrm{PR}$ & - & - & - \\
\hline $\begin{array}{l}\text { Rangeley Lake } \\
\text { Rangeley Stream }\end{array}$ & 01-0505 00 & $\begin{array}{ll}44 & 58 \\
70 & 46\end{array}$ & 90 & 6,250 & 0 & $\mathrm{~d} 30,700$ & $\mathrm{~d} 30,700$ & 2 & $\mathrm{LP}$ & 1882 & $\begin{array}{l}\text { Unton Water Power } \\
\text { Co }\end{array}$ & PA \\
\hline $\begin{array}{l}\text { Ripogenus Lake and others } \\
\text { West Branch Penobscot River }\end{array}$ & $01-0270 \quad 00$ & $\begin{array}{ll}45 & 53 \\
69 & 10\end{array}$ & 1,410 & 243,000 & - & - & 977,000 & 5 & LP & 1917 & $\begin{array}{l}\text { Great Northern } \\
\text { Paper } \mathrm{Co}\end{array}$ & PA \\
\hline $\begin{array}{l}\text { Sabattus Pond } \\
\text { Sabattus R1ver }\end{array}$ & 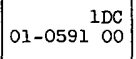 & $\begin{array}{ll}44 & 07 \\
70 & 06\end{array}$ & 35 & 2,918 & - & - & 77,500 & 4 & $\mathrm{PR}$ & - & - & - \\
\hline $\begin{array}{l}\text { Schoodic Lake } \\
\text { Schoodlc Stream }\end{array}$ & $01-0341$ 18H & $\begin{array}{ll}45 & 22 \\
68 & 55\end{array}$ & 43 & 6,989 & - & - & 23,760 & 5 & $P$ & - & $\begin{array}{l}\text { Bangor Hydro- } \\
\text { Electric Co }\end{array}$ & - \\
\hline $\begin{array}{l}\text { Sebago Lake } \\
\text { Presumpscot R1ver }\end{array}$ & $01-063500$ & $\begin{array}{ll}43 & 49 \\
70 & 27\end{array}$ & 436 & 29,200 & o & 223,000 & 223,000 & 5 & MP & 1878 & S P Warren Co & PA \\
\hline $\begin{array}{l}\text { Sebast1cook Lake } \\
\text { East Branch Sebast1cook River }\end{array}$ & $\begin{array}{r}\text { ICE } \\
01-0489 \\
00\end{array}$ & $\begin{array}{ll}44 & 51 \\
69 & 17\end{array}$ & 135 & 4,160 & - & - & 20,800 & 1 & P & 1956 & $\begin{array}{c}\text { Central MaIne Power } \\
\text { Co }\end{array}$ & - \\
\hline $\begin{array}{l}\text { Sebec Lake } \\
\text { Sebec RIver }\end{array}$ & $\mid \begin{array}{r}1 \mathrm{BH} \\
01-0325 \quad 00\end{array}$ & $\begin{array}{ll}45 & 17 \\
69 & 06\end{array}$ & 327 & 7,000 & - & - & 57,400 & 1 & $\mathrm{P}$ & 1962 & $\begin{array}{l}\text { Bangor Hydro- } \\
\text { Electric Co }\end{array}$ & PA \\
\hline $\begin{array}{l}\text { Spednik Lake } \\
\text { St Cro1x River }\end{array}$ & $\left|\begin{array}{rr}1 B A \\
01-0184 & 00\end{array}\right|$ & $\begin{array}{ll}45 & 35 \\
67 & 26\end{array}$ & 417 & 15,600 & - & - & 187,100 & 4 & LP & - & St Cro1x Paper Co & - \\
\hline
\end{tabular}




\begin{tabular}{|c|c|}
\hline $\begin{array}{l}\text { Spencer Lake } \\
\text { L1ttle Spencer Stream }\end{array}$ & $01-044500$ \\
\hline $\begin{array}{l}\text { Squapan Lake } \\
\text { Squapan Stream }\end{array}$ & $01-0160^{1 \mathrm{AD}} \mathrm{DO}$ \\
\hline $\begin{array}{l}\text { Telos Lake } \\
\text { Webster Brook }\end{array}$ & $01-028500$ \\
\hline $\begin{array}{l}\text { Th1rd Machias Lake } \\
\text { Machias River }\end{array}$ & $01-0214^{1 \mathrm{BB}}$ \\
\hline $\begin{array}{l}\text { Thompson Lake } \\
\text { L1ttle Androscoggin River }\end{array}$ & $01-0580$ 1DC \\
\hline $\begin{array}{l}\text { Three Ponds-Milton, Northeast, and } \\
\text { Town House * } \\
\text { Salmon Falls River }\end{array}$ & $01-0720$ ID \\
\hline $\begin{array}{l}\text { Tomah Lake } \\
\text { Tomah Stream }\end{array}$ & $01-0191$ IBA \\
\hline $\begin{array}{l}\text { Unity Pond } \\
\text { Twenty-f1ve Mile Stream }\end{array}$ & 1CE \\
\hline $\begin{array}{l}\text { Upper and Lower R1chardson Lakes } \\
\text { Rap1d R1ver }\end{array}$ & 01-0515 100 \\
\hline $\begin{array}{l}\text { Webber Pond } \\
\text { Sevenm1le Brook }\end{array}$ & $01-0492^{1 C F}$ \\
\hline $\begin{array}{l}\text { Wesserunsett Lake } \\
\text { Tributary to Wesserunsett Stream }\end{array}$ & $01-0480$ OO \\
\hline $\begin{array}{l}\text { West Musquash Lake } \\
\text { West Branch Musquash Stream }\end{array}$ & 01-0192 oc \\
\hline $\begin{array}{l}\text { Wilson Pond } \\
\text { W1lson Stream }\end{array}$ & $01-032000$ \\
\hline $\begin{array}{l}\text { Wilson Pond } \\
\text { W1lson Stream }\end{array}$ & $01-0477$ OO \\
\hline $\begin{array}{l}\text { Wyman Pond } \\
\text { Kennebec R1ver }\end{array}$ & $01-0455^{1 C D}$ OO \\
\hline MARYLAND & \\
\hline $\begin{array}{l}\text { Conow1ngo } \\
\text { Susquehanna River }\end{array}$ & $01-5783^{1 P H} 00$ \\
\hline $\begin{array}{l}\text { Deep Creek } \\
\text { Deep Creek }\end{array}$ & $03-0760$ 3BJ \\
\hline $\begin{array}{l}\text { L1berty } \\
\text { North Branch Patapsco R1ver }\end{array}$ & 1RJ \\
\hline
\end{tabular}

\begin{tabular}{|c|c|c|c|c|}
\hline $\begin{array}{ll}45 & 22 \\
70 & 16\end{array}$ & 48 & 1,510 이 & of & 14,700 \\
\hline $\begin{array}{ll}46 & 33 \\
68 & 20\end{array}$ & 671 & 5,360 & 0 & 58,600 \\
\hline $\begin{array}{ll}46 & 09 \\
69 & 08\end{array}$ & a2 40 & 14,000 & 0 & 116,000 \\
\hline $\begin{array}{ll}45 & 04 \\
67 & 53\end{array}$ & 71 & 2,850 & - & - \\
\hline $\begin{array}{ll}44 & 08 \\
70 & 30\end{array}$ & 460 & 4,365 & 0 & 21,850 \\
\hline $\begin{array}{ll}43 & 25 \\
70 & 59\end{array}$ & 111 & - & 0 & 26,200 \\
\hline $\begin{array}{ll}45 & 25 \\
67 & 37\end{array}$ & 92 & 1,880 & 0 & 18,000 \\
\hline $\begin{array}{ll}44 & 38 \\
69 & 22\end{array}$ & 47 & 2,720 & - & - \\
\hline $\begin{array}{ll}44 & 47 \\
70 & 55\end{array}$ & 509 & 8,370 & 0 & 131,000 \\
\hline $\begin{array}{ll}44 & 24 \\
69 & 40\end{array}$ & 29 & 1,220 & - & - \\
\hline $\begin{array}{ll}44 & 51 \\
69 & 45\end{array}$ & 16 & 1,220 & - & - \\
\hline $\begin{array}{ll}45 & 19 \\
67 & 47\end{array}$ & - & 1,590 & 0 & 6,360 \\
\hline $\begin{array}{ll}45 & 29 \\
69 & 51\end{array}$ & - & 970 & 0 & 8,950 \\
\hline $\begin{array}{ll}44 & 35 \\
70 & 14\end{array}$ & 34 & 770 & - & - \\
\hline $\begin{array}{ll}45 & 04 \\
69 & 54\end{array}$ & 2,625 & - & 148,000 & 208,000 \\
\hline $\begin{array}{ll}39 & 40 \\
76 & 10\end{array}$ & 27,098 & 8,563 & 237,263 & 308,000 \\
\hline $\begin{array}{ll}39 & 31 \\
79 & 23\end{array}$ & 647 & 4,500 & 13,085 & 106,060 \\
\hline $\begin{array}{ll}39 & 23 \\
76 & 53\end{array}$ & 164 & 3,110 & 3,860 & 132,975 \\
\hline
\end{tabular}

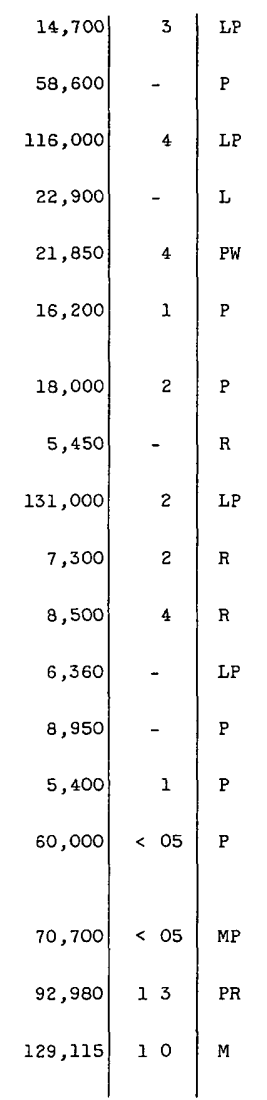

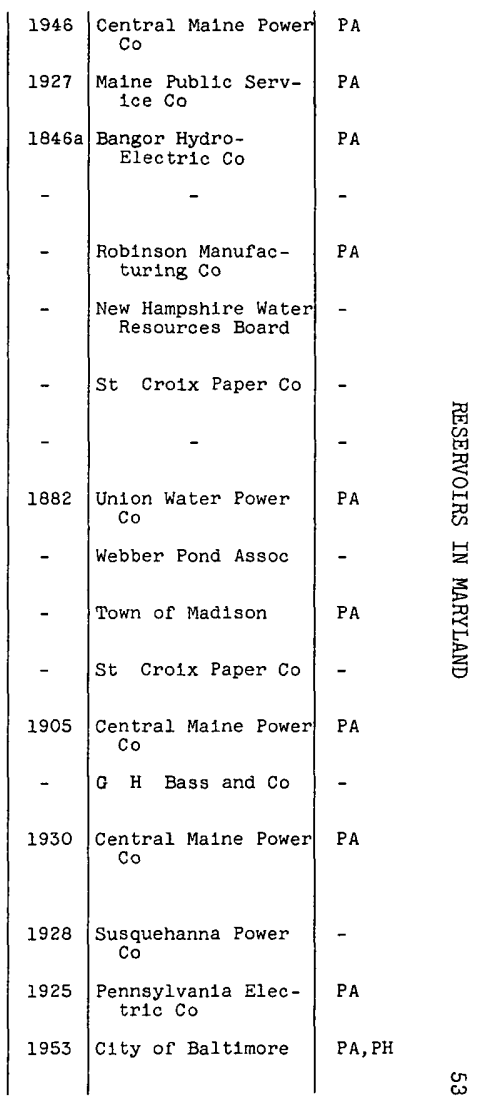


Table 1 --Reservo1rs in the United States completed or under construction as of January 1, 1963, having a usable capac1ty of 5,000 acre-feet or more--Continued [For meaning of symbols under "Use" and "Storage records published," see text]

\begin{tabular}{|c|c|c|c|c|c|c|c|c|c|c|c|c|}
\hline \multirow[b]{2}{*}{$\begin{array}{c}\text { Name of reservoir } \\
\text { and } \\
\text { stream }\end{array}$} & \multicolumn{2}{|c|}{ Location } & \multirow{2}{*}{$\begin{array}{l}\text { Drainage } \\
\text { area } \\
\text { (square } \\
\text { m1les) }\end{array}$} & \multirow[b]{2}{*}{$\begin{array}{c}\text { Surface } \\
\text { area } \\
\text { (acres) }\end{array}$} & \multicolumn{3}{|c|}{ Storage in acre-feet } & \multirow[b]{2}{*}{$\begin{array}{c}\text { Storage } \\
\text { rat10 }\end{array}$} & \multirow[b]{2}{*}{ Use } & \multirow[b]{2}{*}{$\begin{array}{c}\text { Date } \\
\text { com- } \\
\text { pleted }\end{array} \mid$} & \multirow[b]{2}{*}{$\begin{array}{l}\text { Owner } \\
\text { or } \\
\text { operator }\end{array}$} & \multirow{2}{*}{$\begin{array}{l}\text { Storage } \\
\text { records } \\
\text { pub- } \\
\text { lished }\end{array}$} \\
\hline & \begin{tabular}{|c|} 
Basin \\
index and \\
station no
\end{tabular} & $\begin{array}{c}\text { Lat1 tude } \\
\text { and } \\
\text { long1.tude }\end{array}$ & & & Dead & Total & Usable & & & & & \\
\hline MARYLAND--Continued & & & & & & & & & & & & \\
\hline $\begin{array}{l}\text { Loch Raven } \\
\text { Gunpowder Falls }\end{array}$ & $01-583980$ & $\begin{array}{ll}39 & 26 \\
76 & 33\end{array}$ & 303 & 2,391 & 210 & 72,730 & 72,520 & 02 & M & 1914 & C1ty of Baltimore & $\mathrm{PA}, \mathrm{PH}$ \\
\hline $\begin{array}{l}\text { Prettyboy } \\
\text { Gunpowder Falls }\end{array}$ & $\begin{array}{r}\text { IRJ } \\
01-581900\end{array}$ & $\begin{array}{ll}39 & 37 \\
76 & 42\end{array}$ & 80 & 1,498 & 710 & 60,990 & 60,280 & 8 & M & 1933 & City of Baltimore & $\mathrm{PA}, \mathrm{PH}$ \\
\hline $\begin{array}{l}\text { Rocky Gorge } \\
\text { Patuxent River }\end{array}$ & $01-592495$ & $\begin{array}{ll}39 & 07 \\
76 & 52\end{array}$ & 132 & 830 & 307 & $d 19,642$ & 18,106 & 2 & MR & 1954 & $\begin{array}{l}\text { Washington Suburban } \\
\text { San1 tary Comm }\end{array}$ & PA \\
\hline $\begin{array}{l}\text { Savage River } \\
\text { Savage River }\end{array}$ & $\begin{array}{r}1 S A \\
01-597490\end{array}$ & $\begin{array}{ll}39 & 30 \\
79 & 08\end{array}$ & 105 & 366 & 0 & 20,000 & 20,000 & 2 & FRMY & 1952 & $\begin{array}{l}\text { Upper Potomac River } \\
\text { Commission }\end{array}$ & $\mathrm{PA}$ \\
\hline $\begin{array}{c}\text { Triadelphia Lake (Brighton Dam) } \\
\text { Patuxent River } \\
\text { MASSACHUSETTS }\end{array}$ & 01-5916 00 & $\begin{array}{ll}39 & 12 \\
77 & 00\end{array}$ & 786 & 800 & 0 & 20,199 & 20,199 & 3 & MR & 1943 & $\begin{array}{c}\text { Washington Suburban } \\
\text { San1 tary Comm }\end{array}$ & PA \\
\hline $\begin{array}{l}\text { Assawompset, Pocksha, Great Quit- } \\
\text { tacas, and Little Quittacas } \\
\text { Ponds } \\
\text { Nemaskat River }\end{array}$ & $01-107300$ & $\begin{array}{ll}41 & 51 \\
70 & 55\end{array}$ & 260 & 4,166 & - & 45,683 & 5,000 & - & M & - & $\begin{array}{l}\text { Cities of Taunton } \\
\text { and New Bedford }\end{array}$ & - \\
\hline $\begin{array}{l}\text { Barre Falls } \\
\text { Ware RIver }\end{array}$ & $01-1724 \quad 1 \mathrm{GM}$ & $\begin{array}{ll}42 & 26 \\
72 & 02\end{array}$ & 550 & 1,400 & - & - & 24,000 & 35 & $\mathrm{~F}$ & 1958 & Corps of Engineers & PA \\
\hline $\begin{array}{l}\text { Birch Hill } \\
\text { Millers River }\end{array}$ & $01-163500$ & $\begin{array}{ll}42 & 38 \\
72 & 07\end{array}$ & 175 & 3,200 & - & - & 50,000 & 2 & $\mathrm{~F}$ & 1941 & Corps of Engineers & $\mathrm{PA}$ \\
\hline $\begin{array}{l}\text { Borden Brook } \\
\text { Boraen Brook }\end{array}$ & $\begin{array}{r}1 G N \\
01-1819^{1 G N}\end{array}$ & $\begin{array}{ll}42 & 08 \\
72 & 56\end{array}$ & 80 & 214 & o & 7,897 & 7,897 & 7 & MP & 1909 & City of Springfield & PA \\
\hline $\begin{array}{l}\text { Buffumville } \\
\text { Little River }\end{array}$ & $01-124400$ & $\begin{array}{ll}42 & 07 \\
71 & 54\end{array}$ & 265 & 530 & - & - & 12,700 & 4 & $\mathrm{FR}$ & 1958 & Corps of Engineers & PA \\
\hline $\begin{array}{l}\text { Cambridge } \\
\text { Hobbs Brook }\end{array}$ & $01-104300$ & $\begin{array}{ll}42 & 24 \\
71 & 16\end{array}$ & $\begin{array}{ll}6 & 9\end{array}$ & 461 & - & - & 9,275 & - & $M$ & 1897 & C1ty of Cambridge & - \\
\hline $\begin{array}{l}\text { Cobble Mountain } \\
\text { Westfleld Little River }\end{array}$ & $01-182500$ & $\begin{array}{ll}42 & 07 \\
72 & 53\end{array}$ & $\begin{array}{ll}45 & 8\end{array}$ & 1,134 & 26 & 70,000 & 70,000 & 11 & MP & 1931 & City of Springfield & $\mathrm{PA}$ \\
\hline $\begin{array}{l}\text { East Brimfield } \\
\text { Quinebaug River }\end{array}$ & $01-1233^{1 F G}$ & $\begin{array}{ll}42 & 07 \\
72 & 08\end{array}$ & $\begin{array}{ll}67 & 5\end{array}$ & 2,300 & - & - & 31,800 & 4 & FRW & 1960 & Corps of Engineers & $P A$ \\
\hline $\begin{array}{l}\text { Hodges V1llage } \\
\text { French River }\end{array}$ & $01-124300$ & $\begin{array}{ll}42 & 07 \\
71 & 53\end{array}$ & 310 & 740 & - & - & 13,200 & 3 & $\mathrm{~F}$ & 1959 & Corps of Engineers & $\mathrm{PA}$ \\
\hline
\end{tabular}

.


Knightville

1d River

Lake Cochichewich Merrimack River tributary

Lake Cochituate

Sudbury River tributary

North Watuppa Pond

Quequechan River

Otis

Asnebumskit Brook

Putnamville

Ipsw1ch RIver c/

Quabbin

Swift River

South Watuppa Pond

Quequechan R1ve

Sudbury

udbury River

Tighe-Carmody
Manhan River

Tully

Fest Branch Tully R1ver

Wachusett

South Branch Nashua River

West Hill
West River

Westville

Wuinebaug River

\section{MICHIGAN}

Alcona Pond (Bamfield)

Au Sable River

Belleville Lake

\section{Huron River}

Middle Branch Ontonagon River See footnotes at end of table

\begin{tabular}{|c|c|}
\hline $\mid \begin{array}{r}1 \mathrm{GN} \\
01-1790 \quad 00\end{array}$ & $\begin{array}{ll}42 & 17 \\
72 & 5\end{array}$ \\
\hline $01-100650$ & $\begin{array}{ll}42 & 4 \\
71 & 0\end{array}$ \\
\hline $01-0984 \stackrel{1 E G}{50}$ & $\begin{array}{ll}421 \\
712\end{array}$ \\
\hline $01-1091_{10}^{1 \mathrm{FC}}$ & $\begin{array}{l}41 \\
71\end{array}$ \\
\hline$-{ }^{1 \mathrm{GO}}$ & $\begin{array}{l}42 \\
73\end{array}$ \\
\hline $01-095350$ & $\begin{array}{l}422 \\
715\end{array}$ \\
\hline $01-101800$ & $\begin{array}{l}42 \\
70\end{array}$ \\
\hline $01-1750$ 1GM & \\
\hline $01-109120$ & \\
\hline $01-097490$ & 42 \\
\hline $01-1716 \stackrel{1 \mathrm{GM}}{00}$ & $\begin{array}{l}42 \\
72\end{array}$ \\
\hline $01-164500$ & 4 \\
\hline $01-095450$ & $\begin{array}{l}42 \\
71\end{array}$ \\
\hline $01-1111$ OFE & $\begin{array}{l}42 \\
71\end{array}$ \\
\hline $01-1235^{1 F G}$ & $\begin{array}{l}420 \\
720\end{array}$ \\
\hline $04-1370$ OHG & $\begin{array}{l}44 \\
83\end{array}$ \\
\hline $04-175000$ & $\begin{array}{l}42 \\
83\end{array}$ \\
\hline $\begin{array}{r}4 \mathrm{BA} \\
04-0340 \quad 00\end{array}$ & 89 \\
\hline
\end{tabular}

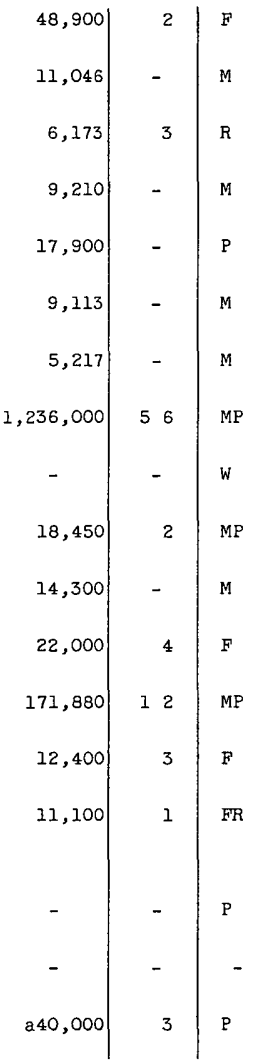

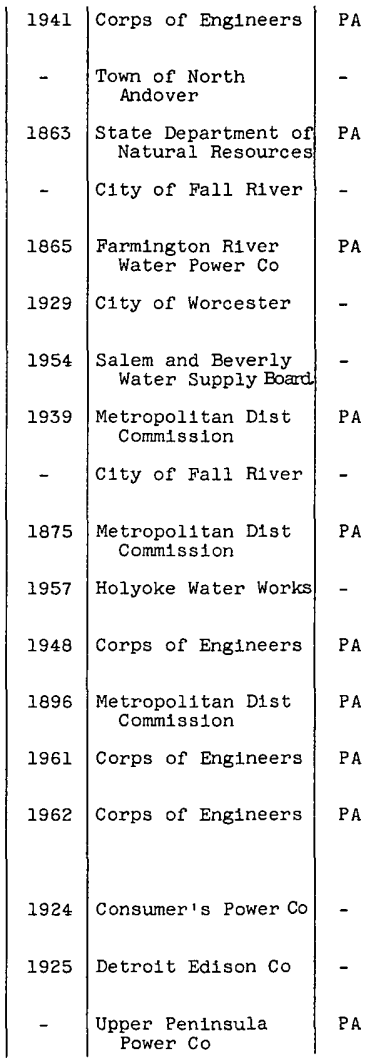




\begin{tabular}{|c|c|c|c|c|c|c|c|c|c|c|c|c|}
\hline \multirow[b]{2}{*}{$\begin{array}{l}\text { Name of reservo1r } \\
\text { and } \\
\text { stream }\end{array}$} & \multicolumn{2}{|c|}{ Location } & \multirow{2}{*}{$\begin{array}{c}\text { Drainage } \\
\text { area } \\
\text { (square } \\
\text { miles) }\end{array}$} & \multirow[b]{2}{*}{$\begin{array}{c}\text { Surface } \\
\text { area } \\
\text { (acres) }\end{array}$} & \multicolumn{3}{|c|}{ Storage in acre-feet } & \multirow[b]{2}{*}{$\begin{array}{c}\text { Storage } \\
\text { rat10 }\end{array}$} & \multirow[b]{2}{*}{ Use } & \multirow[b]{2}{*}{$\begin{array}{c}\text { Date } \\
\text { com- } \\
\text { pleted }\end{array}$} & \multirow[b]{2}{*}{$\begin{array}{l}\text { Owner } \\
\text { or } \\
\text { operator }\end{array}$} & \multirow{2}{*}{$\begin{array}{l}\text { Storage } \\
\text { records } \\
\text { pub- } \\
\text { lished }\end{array}$} \\
\hline & \begin{tabular}{|c|} 
Basin \\
index and \\
station no \\
\end{tabular} & $\begin{array}{c}\text { Lat1 tude } \\
\text { and } \\
\text { long1tude }\end{array}$ & & & Dead & Total & Usable & & & & & \\
\hline MICHIGAN--Continued & & & & & & & & & & & & \\
\hline $\begin{array}{l}\text { Brule * } \\
\text { Brule River }\end{array}$ & $\begin{array}{r}4 \mathrm{CJ} \\
04-062010\end{array}$ & $\begin{array}{ll}45 & 57 \\
88 & 13\end{array}$ & 1,050 & 774 & - & 18,000 & - & - & $\mathrm{P}$ & 1919 & $\begin{array}{l}\text { Wisconsin-Michigan } \\
\text { Power Co }\end{array}$ & - \\
\hline $\begin{array}{l}\text { Chalk Hill }{ }^{*} \\
\text { Menominee River }\end{array}$ & $04-066020$ & $\begin{array}{ll}45 & 31 \\
87 & 48\end{array}$ & 3,321 & 765 & - & $a 8,000$ & - & - & $\mathrm{P}$ & 1927 & $\begin{array}{l}\text { Wisconsin-Michigan } \\
\text { Power Co }\end{array}$ & - \\
\hline $\begin{array}{l}\text { Cleveland Cliffs Basin } \\
\text { Au Train River }\end{array}$ & $04-0447 \quad 20$ & $\begin{array}{ll}46 & 19 \\
86 & 51\end{array}$ & 80 & 1,949 & - & - & 12,300 & - & $\mathrm{P}$ & 1931 & $\begin{array}{l}\text { Cleveland-Cliffs } \\
\text { Iron Co }\end{array}$ & - \\
\hline $\begin{array}{l}\text { Cooke Dam Basin } \\
\text { Au Sable River }\end{array}$ & $04-1374 \quad 00$ & $\begin{array}{ll}44 & 28 \\
83 & 34\end{array}$ & 1,641 & 1,800 & - & - & $>5,000$ & - & $\mathrm{P}$ & 1911 & Consumer's Power Co & - \\
\hline $\begin{array}{l}\text { Croton Pond } \\
\text { Muskegon River }\end{array}$ & $\begin{array}{r}4 \mathrm{GC} \\
04-121950\end{array}$ & $\begin{array}{ll}43 & 26 \\
85 & 40\end{array}$ & 2,224 & 1,380 & - & - & $>5,000$ & - & $\mathrm{P}$ & 1907 & Consumer's Power $\mathrm{Co}$ & - \\
\hline $\begin{array}{l}\text { Dead River Storage Basin (Ho1st Darn) } \\
\text { Dead R1ver }\end{array}$ & $04-0435$ OBD & $\begin{array}{ll}46 & 34 \\
87 & 34\end{array}$ & 141 & 4,236 & - & - & $a 55,300$ & - & $P$ & 1916 & $\begin{array}{l}\text { Cleveland-Cliffs } \\
\text { Iron Co }\end{array}$ & - \\
\hline $\begin{array}{l}\text { Deer Lake Basin } \\
\text { Carp R1ver }\end{array}$ & $\begin{array}{r}4 \mathrm{BE} \\
04-0443 \quad 40\end{array}$ & $\begin{array}{ll}46 & 32 \\
87 & 40\end{array}$ & 363 & al, 700 & - & - & a22, 500 & - & $P$ & 1912 & $\begin{array}{l}\text { Cleveland-Cliffs } \\
\text { Iron Co }\end{array}$ & - \\
\hline 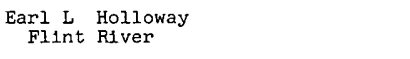 & $04-147000$ & $\begin{array}{ll}43 & 07 \\
83 & 30\end{array}$ & 543 & 1,973 & - & - & 17,700 & 09 & MW & 1953 & C1ty of Flint & PA \\
\hline $\begin{array}{l}\text { Fletcher Pond } \\
\text { Upper South Branch Thunder Bay } \\
\text { R1ver }\end{array}$ & $04-1329 \begin{array}{r}4 \mathrm{HF} \\
05\end{array}$ & $\begin{array}{ll}45 & 02 \\
83 & 47\end{array}$ & 171 & 8,560 & 4,500 & 44,600 & 40,100 & 5 & $\mathrm{P}$ & 1930 & Alpena Power Co & - \\
\hline $\begin{array}{l}\text { Foote Basin } \\
\text { Au Sable River }\end{array}$ & $04-137500$ & $\begin{array}{ll}44 & 26 \\
83 & 26\end{array}$ & 1,664 & 1,850 & - & - & $>5,000$ & - & $\mathrm{P}$ & 1918 & Consumer's Power Cs & - \\
\hline $\begin{array}{l}\text { Ford Lake } \\
\text { Huron River }\end{array}$ & $04-1749 \quad \begin{array}{r}4 \mathrm{~KB} \\
00\end{array}$ & $\begin{array}{ll}42 & 12 \\
83 & 33\end{array}$ & 812 & 917 & - & 16,500 & - & - & $P$ & 1932 & Ford Motor Co & - \\
\hline $\begin{array}{l}\text { Hardy Lake } \\
\text { Muskegon River }\end{array}$ & $\begin{array}{r}4 \mathrm{GC} \\
04-121750\end{array}$ & $\begin{array}{ll}43 & 29 \\
85 & 38\end{array}$ & 1,851 & 3,970 & - & - & $>5,000$ & - & $P$ & 1931 & Consumer's Power co & - \\
\hline $\begin{array}{l}\text { Hodenpyl backwater } \\
\text { Manistee R1ver }\end{array}$ & $04-124020$ & $\begin{array}{ll}44 & 22 \\
85 & 49\end{array}$ & 1,018 & 2,025 & - & - & $>5,000$ & - & $\mathrm{P}$ & 1925 & Consumer's Power co & - \\
\hline $\begin{array}{l}\text { Hubbard Lake } \\
\text { Lower South Branch Thunder Bay } \\
\text { R1ver }\end{array}$ & $04-134500$ & $\begin{array}{ll}44 & 52 \\
83 & 36\end{array}$ & 146 & 8,800 & 4,200 & 34,200 & 30,000 & 4 & $\mathrm{PR}$ & 1915 & Alpena Power Co & - \\
\hline $\begin{array}{l}\text { Lake Allegan (Calkins Dam) } \\
\text { Kalamazoo River }\end{array}$ & $04-108000$ & $\begin{array}{ll}42 & 34 \\
85 & 57\end{array}$ & 1,540 & 1,600 & - & 17,200 & - & - & P & 1936 & City of Allegan & - \\
\hline
\end{tabular}


Lake Gogeblc
West Branch Ontonagon River

Michlgamme (Way Dam)

Morrow Lake
Kalamazoo River

Norway Point

Thunder Bay River

Peavy Pond (Peavy Falls)
Michigamme River

Sanford Lake

abawassee River

Schweltzer

Schweltzer Creek

Silver Lake

Tippy backwater
Manistee River

Wh1 te Rapids *

Wixom Lake (Endenville Dam

Tittabawassee River

MINNESOTA

Bass Lake (No 14-29)
Bass Lake Outlet

Bass Lake Outlet

Bemidj1 Lake
Mississippi River

Big Birch Lake (No 7-1)
Birch Lake Outlet

Big Stone Lake (No 6-31)

Minnesota River

B1rch Lake

Kawishiwi River

Boulder Lake

Carlos Lake (No 6-3)
Long Prairie River

See footnotes at end of table

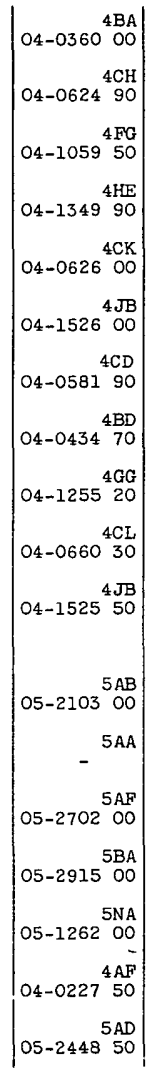

\begin{tabular}{|c|c|c|}
\hline $\begin{array}{ll}46 & 35 \\
89 & 33\end{array}$ & 162 & 14,080 \\
\hline $\begin{array}{ll}46 & 10 \\
88 & 14\end{array}$ & 642 & 7,000 \\
\hline $\begin{array}{ll}42 & 17 \\
85 & 29\end{array}$ & 1,000 & 1,000 \\
\hline $\begin{array}{ll}45 & 06 \\
83 & 31\end{array}$ & 1,260 & 1,700 \\
\hline $\begin{array}{ll}45 & 59 \\
88 & 13\end{array}$ & 710 & 3,160 \\
\hline $\begin{array}{ll}43 & 41 \\
84 & 23\end{array}$ & 1,020 & 1,526 \\
\hline $\begin{array}{ll}46 & 25 \\
87 & 39\end{array}$ & 231 & b360 \\
\hline $\begin{array}{ll}46 & 39 \\
87 & 50\end{array}$ & 24 & - \\
\hline $\begin{array}{ll}44 & 15 \\
85 & 56\end{array}$ & 1,451 & 1,540 \\
\hline $\begin{array}{ll}45 & 29 \\
87 & 48\end{array}$ & 3,325 & 445 \\
\hline $\begin{array}{ll}43 & 49 \\
84 & 22\end{array}$ & 985 & 2,178 \\
\hline $\begin{array}{ll}47 & 17 \\
93 & 37\end{array}$ & 24 & 2,644 \\
\hline $\begin{array}{ll}47 & 29 \\
94 & 44\end{array}$ & 1,088 & 6,765 \\
\hline $\begin{array}{ll}45 & 47 \\
94 & 53\end{array}$ & 35 & 2,143 \\
\hline $\begin{array}{ll}45 & 18 \\
96 & 27\end{array}$ & 1,160 & - \\
\hline $\begin{array}{ll}47 & 49 \\
91 & 47\end{array}$ & - & 5,630 \\
\hline $\begin{array}{ll}47 & 03 \\
92 & 12\end{array}$ & 60 & 4,480 \\
\hline $\begin{array}{ll}45 & 59 \\
95 & 20\end{array}$ & 226 & 6,433 \\
\hline
\end{tabular}

-
-
-
-
-
-
-
-
-
-
-
-

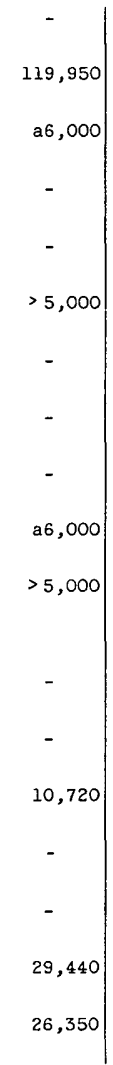

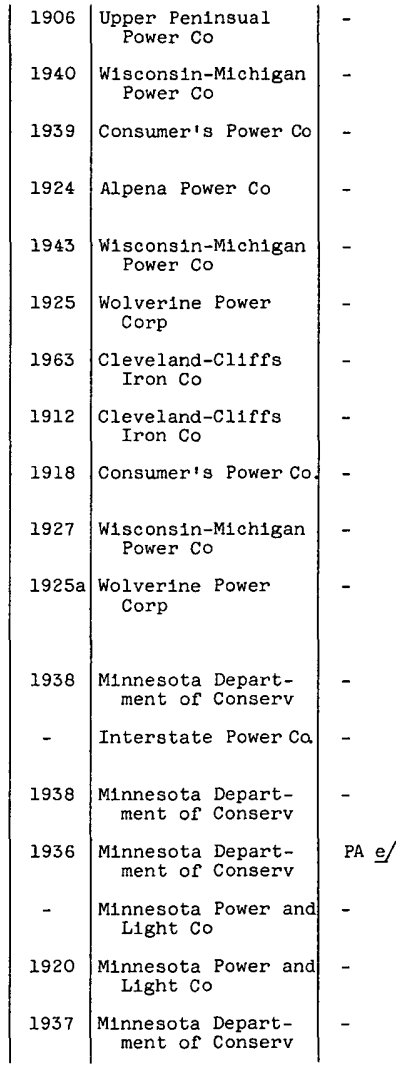

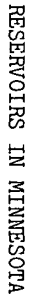


Table I --Reservo1rs in the United States completed or under construction as of January 1, 1963, having a usable capac1ty of 5,000 acre-feet or more--Cont1nued

\begin{tabular}{|c|c|c|c|c|c|c|c|c|c|c|c|c|}
\hline \multirow{2}{*}{$\begin{array}{c}\text { Name of reservolr } \\
\text { and } \\
\text { stream }\end{array}$} & \multicolumn{2}{|c|}{ Location } & \multirow{2}{*}{$\begin{array}{l}\text { Dralnage } \\
\text { area } \\
\text { (square } \\
\text { m1les) }\end{array}$} & \multirow[b]{2}{*}{$\begin{array}{c}\text { Surface } \\
\text { area } \\
\text { (acres) }\end{array}$} & \multicolumn{3}{|c|}{ Storage in acre-feet } & \multirow[b]{2}{*}{$\begin{array}{c}\text { Storage } \\
\text { rat10 }\end{array}$} & \multirow[b]{2}{*}{ Use } & \multirow[b]{2}{*}{$\begin{array}{c}\text { Date } \\
\text { com- } \\
\text { pleted }\end{array}$} & \multirow[b]{2}{*}{$\begin{array}{l}\text { Owner } \\
\text { or } \\
\text { operator }\end{array}$} & \multirow{2}{*}{$\begin{array}{l}\text { Storage } \\
\text { records } \\
\text { pub- } \\
\text { lished }\end{array}$} \\
\hline & \begin{tabular}{|c|} 
Basin \\
1ndex and \\
station no
\end{tabular} & $\begin{array}{l}\text { Lat1 tude } \\
\text { and } \\
\text { long1tude }\end{array}$ & & & Dead & Total & Usable & & & & & \\
\hline $\begin{array}{l}\text { MINNESOTA--Continued } \\
\text { Cass Lake } \\
\text { M1ss1ssippi River }\end{array}$ & $05-200650$ & $\begin{array}{ll}47 & 25 \\
94 & 29\end{array}$ & 1,088 & 20,347 & - & - & $\mathrm{d} 83,420$ & - & $\mathrm{c}$ & 1928 & U S Forest Service & - \\
\hline $\begin{array}{l}\text { Dead Lake (No 9-4) } \\
\text { Dead R1ver }\end{array}$ & $\begin{array}{r}50 \mathrm{~B} \\
05-030390\end{array}$ & $\begin{array}{ll}46 & 28 \\
95 & 45\end{array}$ & 100 & 7,708 & - & 30,830 & 23,890 & - & $\mathrm{CR}$ & 1938 & $\begin{array}{l}\text { Minnesota Depart- } \\
\text { ment of Conserv }\end{array}$ & - \\
\hline $\begin{array}{l}\text { Deer Lake (No 14-7) } \\
\text { Deer Lake Outlet }\end{array}$ & 05-1318 500 & $\begin{array}{ll}47 & 51 \\
93 & 26\end{array}$ & 38 & 1,652 & 2,590 & 9,750 & 7,160 & - & $\mathrm{CR}$ & 1938 & $\begin{array}{l}\text { Minnesota Depart- } \\
\text { ment of Conserv }\end{array}$ & - \\
\hline $\begin{array}{l}\text { Deer Lake (No 14-23) } \\
\text { Deer RIver }\end{array}$ & $05-209000$ & $\begin{array}{ll}47 & 23 \\
93 & 43\end{array}$ & 22 & 4,157 & 6,240 & 16,880 & 10,640 & - & $\mathrm{CR}$ & 1938 & $\begin{array}{l}\text { Minnesota Depart- } \\
\text { ment of Conserv }\end{array}$ & - \\
\hline $\begin{array}{l}\text { Fish Lake } \\
\text { Beaver River }\end{array}$ & $\begin{array}{r}4 A F \\
04-022950\end{array}$ & $\begin{array}{ll}46 & 57 \\
92 & 17\end{array}$ & 47 & 4,480 & 0 & 39,680 & 39,680 & - & $\mathrm{P}$ & 1911 & $\underset{\text { Light Co }}{\text { Minnesota Power and }}$ & - \\
\hline $\begin{array}{l}\text { Gull Lake } \\
\text { Gull River }\end{array}$ & $\begin{array}{r}5 \mathrm{AC} \\
05-246500\end{array}$ & $\begin{array}{ll}46 & 25 \\
94 & 21\end{array}$ & 287 & 13,139 & - & - & $\mathrm{b} 26,020$ & 04 & FMNR & 1913 & Corps of Englneers & PA \\
\hline $\begin{array}{l}\text { Height of Land Lake (No 9-9) } \\
\text { Otter Tail River }\end{array}$ & $\begin{array}{r}50 B \\
05-029500\end{array}$ & $\begin{array}{ll}46 & 53 \\
95 & 38\end{array}$ & 218 & 3,941 & of & 20,100 & 20,100 & - & $\mathrm{CR}$ & 1938 & $\begin{array}{l}\text { Minnesota Depart- } \\
\text { ment of Conserv }\end{array}$ & $\mathrm{PA} e /$ \\
\hline $\begin{array}{l}\text { Heron Lake (No } 3-1 \text { ) } \\
\text { Heron Lake Outlet }\end{array}$ & $05-474990$ & $\begin{array}{ll}43 & 48 \\
95 & 16\end{array}$ & 457 & 4,000 & of & 19,360 & 19,360 & - & $C R$ & 1939 & $\begin{array}{l}\text { Minnesota Depart- } \\
\text { ment of Conserv }\end{array}$ & - \\
\hline $\begin{array}{l}\text { Ida Lake (No 6-9) } \\
\text { Ida Lake Outlet }\end{array}$ & $\begin{array}{r}5 A D \\
05-244650\end{array}$ & $\begin{array}{ll}45 & 57 \\
95 & 25\end{array}$ & 46 & 4,643 & - & - & 28,850 & - & $\mathrm{CR}$ & 1937 & $\begin{array}{l}\text { Minnesota Depart- } \\
\text { ment of Conserv }\end{array}$ & - \\
\hline $\begin{array}{l}\text { Island Lake } \\
\text { Cloquet River }\end{array}$ & $\begin{array}{r}4 \mathrm{AF} \\
04-022800\end{array}$ & $\begin{array}{ll}46 & 60 \\
92 & 14\end{array}$ & 486 & 11,200 & 0 & 171,520 & 171,520 & 73 & $\mathrm{P}$ & 1909 & $\underset{\text { Minnesota Power and }}{\text { Light Co }}$ & - \\
\hline $\begin{array}{l}\text { Lacqui Parle } \\
\text { Minnesota River }\end{array}$ & $05-2950 \quad 00$ & $\begin{array}{ll}45 & 02 \\
95 & 52\end{array}$ & 6,100 & 12,800 & 20,100 & 123,000 & 102,900 & 22 & $\mathrm{CF}$ & 1937 & Corps of Engineers & - \\
\hline $\begin{array}{l}\text { Lake Traverse (White Rock Dam)* } \\
\text { Bo1s de Sioux River }\end{array}$ & $\begin{array}{r}50 \mathrm{~A} \\
05-049900\end{array}$ & $\begin{array}{ll}45 & 52 \\
96 & 34\end{array}$ & 1,160 & 22,975 & b112,500 & 249,500 & 137,000 & 267 & $\mathrm{~F}$ & 1941 & Corps of Engineers & $\mathrm{PA}$ \\
\hline $\begin{array}{l}\text { Lake of the Woods } \\
\text { WInnipeg RIver }\end{array}$ & $05-1410 \quad 00$ & $\begin{array}{ll}49 & 46 \\
94 & 33\end{array}$ & 27,170 & 950,400 & of & $6,177,600$ & $6,177,600$ & - & FPRW & 1905 & - & PB \\
\hline $\begin{array}{l}\text { Leech Lake } \\
\text { Leech Lake River }\end{array}$ & $\begin{array}{r}5 \mathrm{AA} \\
05-206000\end{array}$ & $\begin{array}{ll}47 & 12 \\
94 & 19\end{array}$ & 1,163 & 160,545 & - & - & 689,780 & bl 5 & FMNR & 1902 & Corps of Engineers & PA \\
\hline $\begin{array}{l}\text { Lizzle Lake (No 9-43) } \\
\text { Pelican River }\end{array}$ & $\begin{array}{r}50 B \\
05-040250\end{array}$ & $\begin{array}{ll}46 & 37 \\
96 & 02\end{array}$ & 274 & 6,242 & - & - & 27,196 & - & $C R$ & 1938 & $\begin{array}{l}\text { Minnesota Depart- } \\
\text { ment of Conserv }\end{array}$ & - \\
\hline $\begin{array}{l}\text { Marsh Lake } \\
\text { Minnesota River }\end{array}$ & $\left|\begin{array}{r}5 B A \\
05-2930 \\
10\end{array}\right|$ & $\begin{array}{ll}45 & 11 \\
96 & 06\end{array}$ & 2,703 & 8,600 & 0 & 35,000 & 35,000 & 19 & $\mathrm{CP}$ & 1938 & Corps of Engineers & - \\
\hline
\end{tabular}


Melissa Lake (No 9-18)

lican River

M1lle Lacs Lake
Rum River

1
Miltona Lake (No 6-15)
Miltona Lake Out1et

Mud Lake
Thief River

or Namakan Chain (Kettle Falls) Rainy River

Onamia Lake (No 7-7)

Rum River

Orwell
Otter Ta1l River

Osakis Lake (No 7-23)

Otter Ta11 Lake (No 9-19)

Otter Ta11 River

Pelican Lake (No 9-36)
Pelican River

Pelican Lake (No 16-4)

Pelican Lake (No 10-43)

Pine Lake (No 9-20)

Pine River
PIne Rive

Pokegama

Mistsippl River

Potato Lake (No 12-17)

Rainy Lake

Raıny River

Red Lake
Red Lake River

See footnotes at end of table

\begin{tabular}{|c|c|c|c|c|c|c|c|c|c|c|c|}
\hline $\begin{array}{r}50 \mathrm{~B} \\
05-039000\end{array}$ & $\begin{array}{ll}46 & 43 \\
95 & 55\end{array}$ & 123 & 1,791 & 2,686 & 19,701 & b13,790 & 5 & $\mathrm{CR}$ & 1938 & $\begin{array}{c}\text { Minnesota Depart- } \\
\text { ment of Conserv }\end{array}$ & $\mathrm{PA}$ e/ \\
\hline $\begin{array}{r}5 \text { AH } \\
05-2840 \text { OO }\end{array}$ & $\begin{array}{ll}46 & 08 \\
93 & 46\end{array}$ & 400 & 132,520 & $3,700,000$ & $3,965,000$ & 265,000 & - & $\mathrm{R}$ & 1939 & State of Minnesota & $\mathrm{PA}$ e/ \\
\hline $\begin{array}{r}5 \mathrm{AD} \\
05-2446 \quad 30\end{array}$ & $\begin{array}{ll}46 & 02 \\
95 & 24\end{array}$ & 35 & 5,322 & - & - & a6, 920 & - & $\mathrm{CR}$ & 1938 & $\begin{array}{l}\text { Minnesota Depart- } \\
\text { ment of Conserv }\end{array}$ & $\mathrm{PA}$ e/ \\
\hline $\begin{array}{r}\text { 5PA } \\
05-075800\end{array}$ & $\begin{array}{ll}\text { a } 48 & 20 \\
\text { a96 } & 00\end{array}$ & - & 28,120 & of & 49,730 & 49,730 & - & c & - & $\begin{array}{l}\text { U S Sports Fish- } \\
\text { eries and W1ld- } \\
\text { Ilfe Service }\end{array}$ & - \\
\hline$-{ }^{5 \mathrm{NC}}$ & $\begin{array}{ll}48 & 30 \\
92 & 38\end{array}$ & 7,200 & 66,600 & 142,600 & - & 575,700 & 2 & NPR & 1914 & $\begin{array}{l}\text { Minnesota and } \\
\text { Ontario Paper Co } \\
\text { and Subsidiaries }\end{array}$ & PB \\
\hline $05-284480$ & $\begin{array}{ll}46 & 05 \\
93 & 40\end{array}$ & 430 & 2,836 & - & - & $a 7,080$ & - & $\mathrm{CR}$ & 1938 & $\begin{array}{l}\text { Minnesota Depart- } \\
\text { ment of Conserv }\end{array}$ & $\mathrm{PA}$ ef \\
\hline $\begin{array}{r}50 \mathrm{~B} \\
05-045950\end{array}$ & $\begin{array}{ll}46 & 13 \\
96 & 11\end{array}$ & 1,830 & $\mathrm{bl}, 110$ & 210 & 20,400 & b19,400 & b 07 & - & 1953 & Corps of Engineers & PA \\
\hline $\begin{array}{r}5 \mathrm{AF} \\
05-270100\end{array}$ & $\begin{array}{ll}45 & 54 \\
95 & 05\end{array}$ & 40 & 6,587 & - & - & 13,065 & - & $\mathrm{CR}$ & 1939 & $\begin{array}{l}\text { Minnesota Depart- } \\
\text { ment of Conserv }\end{array}$ & - \\
\hline $\begin{array}{r}50 \mathrm{~B} \\
05-030400\end{array}$ & $\begin{array}{ll}46 & 22 \\
95 & 44\end{array}$ & 1,050 & 14,745 & - & - & b34, 969 & 5 & $\mathrm{CR}$ & 1937 & $\begin{array}{l}\text { Minnesota Depart- } \\
\text { ment of Conserv }\end{array}$ & - \\
\hline $\begin{array}{r}50 B \\
05-040100\end{array}$ & $\begin{array}{lll}46 & 40 \\
96 & 01\end{array}$ & 214 & 4,200 & - & - & all, 700 & - & $C R$ & 1938 & $\begin{array}{l}\text { Minnesota Depart- } \\
\text { ment of Conserv }\end{array}$ & - \\
\hline $05-129100$ & $\begin{array}{ll}48 & 02 \\
92 & 50\end{array}$ & 70 & 11,800 & - & - & b39,769 & - & $\mathrm{CR}$ & 1938 & $\begin{array}{l}\text { Minnesota Depart- } \\
\text { ment of Conserv }\end{array}$ & - \\
\hline $\begin{array}{r}5 \mathrm{AC} \\
05-231500\end{array}$ & $\begin{array}{ll}46 & 37 \\
94 & 11\end{array}$ & - & 9,835 & o) & d34, 420 & $\mathrm{~d} 34,420$ & - & $\mathrm{R}$ & - & $\begin{array}{l}\text { Pelican Lakes } \\
\text { Village }\end{array}$ & PA \\
\hline $\begin{array}{r}50 \mathrm{~B} \\
05-030280\end{array}$ & $\begin{array}{ll}46 & 36 \\
95 & 30\end{array}$ & 585 & 4,820 & - & - & 15,788 & - & $\mathrm{CR}$ & 1938 & $\begin{array}{l}\text { Minnesota Depart- } \\
\text { ment of Conserv }\end{array}$ & - \\
\hline $\begin{array}{r}5 \mathrm{AC} \\
05-230500\end{array}$ & $\begin{array}{ll}46 & 40 \\
94 & 07\end{array}$ & 562 & 15,190 & - & - & 118,710 & b 4 & FMNR & 1886 & Corps of Engineers & PA \\
\hline $\begin{array}{r}5 A B \\
05-21,0500\end{array}$ & $\begin{array}{ll}47 & 16 \\
93 & 35\end{array}$ & 3,265 & 22,080 & - & - & 81,720 & b 7 & FMNR & 1889 & Corps of Engineers & PA \\
\hline $\begin{array}{r}5 A D \\
05-243600\end{array}$ & $\begin{array}{ll}46 & 59 \\
95 & 02\end{array}$ & 210 & 2,054 & o) & 8,216 & 8,216 & - & $\mathrm{CR}$ & 1939 & $\begin{array}{l}\text { M1nnesota Depart- } \\
\text { ment of Conserv }\end{array}$ & - \\
\hline $\begin{array}{r}5 \mathrm{NC} \\
05-129400\end{array}$ & $\begin{array}{ll}48 & 36 \\
93 & 24\end{array}$ & 14,900 & 221,000 & - & - & 734,500 & 2 & NPR & 1909 & $\begin{array}{l}\text { Minnesota and } \\
\text { Ontar1o Paper Co } \\
\text { and Subsidiarles }\end{array}$ & PB \\
\hline $\begin{array}{r}5 P A \\
05-0740 \text { OO }\end{array}$ & $\begin{array}{ll}42 & 57 \\
95 & 17\end{array}$ & 1,950 & $\mathrm{~b} 288,800$ & - & $3,270,000$ & $1,905,000$ & - & FMR & 1931 & Corps of Engineers & PA \\
\hline
\end{tabular}

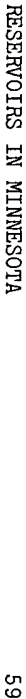


Table 1 --Reservo1rs in the United States completed or under construction as of January 1, 1963, having a usable capacity of 5,000 acre-feet or more--Continued [For meaning of symbols under "Use" and "Storage records published," see text]

\begin{tabular}{|c|c|c|c|c|c|c|c|c|c|c|c|c|}
\hline \multirow[b]{2}{*}{$\begin{array}{c}\text { Name of reservoir } \\
\text { and } \\
\text { stream }\end{array}$} & \multicolumn{2}{|c|}{ Location } & \multirow{2}{*}{$\begin{array}{c}\text { Drainage } \\
\text { area } \\
\text { (square } \\
\text { m1les) }\end{array}$} & \multirow[b]{2}{*}{$\begin{array}{c}\text { Surface } \\
\text { area } \\
\text { (acres) }\end{array}$} & \multicolumn{3}{|c|}{ Storage in acre-feet } & \multirow[b]{2}{*}{$\begin{array}{c}\text { Storage } \\
\text { rat10 }\end{array}$} & \multirow[b]{2}{*}{ Use } & \multirow[b]{2}{*}{$\begin{array}{c}\text { Date } \\
\text { com- } \\
\text { pleted }\end{array}$} & \multirow[b]{2}{*}{$\begin{array}{l}\text { Owner } \\
\text { or } \\
\text { operator }\end{array}$} & \multirow{2}{*}{$\begin{array}{l}\text { Storage } \\
\text { records } \\
\text { pub- } \\
\text { 11shed }\end{array}$} \\
\hline & $\begin{array}{c}\text { Basin } \\
\text { 1ndex and } \\
\text { station no }\end{array}$ & $\begin{array}{l}\text { Latitude } \\
\text { and } \\
\text { longitude }\end{array}$ & & & Dead & Total & Usable & & & & & \\
\hline \multicolumn{13}{|l|}{ MINNESOTA--Continued } \\
\hline $\begin{array}{l}\text { Rice Lake } \\
\text { Beaver River }\end{array}$ & $\begin{array}{r}4 \mathrm{AF} \\
04-022900\end{array}$ & $\begin{array}{ll}46 & 55 \\
92 & 10\end{array}$ & 25 & - & 0 & 9,600 & 9,600 & - & $\mathrm{P}$ & 1909 & $\begin{array}{l}\text { Minnesota Power and } \\
\text { Light Co }\end{array}$ & - \\
\hline $\begin{array}{l}\text { Rush Lake (No 9-26) } \\
\text { Otter Tail River }\end{array}$ & $\begin{array}{r}508 \\
05-030350\end{array}$ & $\begin{array}{ll}46 & 29 \\
95 & 34\end{array}$ & 700 & 5,233 & 0 & b24,540 & $\mathrm{b} 24,540$ & - & $\mathrm{CR}$ & 1937 & $\begin{array}{l}\text { Minnesota Depart- } \\
\text { ment of Conserv }\end{array}$ & - \\
\hline $\begin{array}{l}\text { St Croix Falls * } \\
\text { St Crolx River }\end{array}$ & $05-3404 \quad 95$ & $\begin{array}{ll}45 & 25 \\
92 & 39\end{array}$ & 5,930 & 840 & o) & $\mathrm{d} 5,080$ & $d 5,080$ & $<005$ & $\mathrm{P}$ & 1905 & $\begin{array}{l}\text { Northern States } \\
\text { Power Co }\end{array}$ & - \\
\hline $\begin{array}{l}\text { Sandy Lake } \\
\text { Sandy River }\end{array}$ & $\begin{array}{r}5 A B \\
05-218500\end{array}$ & $\begin{array}{ll}46 & 47 \\
93 & 19\end{array}$ & 421 & 10,643 & - & - & 73,330 & b 3 & FMNR & 1911 & Corps of Engineers & PA \\
\hline $\begin{array}{l}\text { Sturgeon River (No 16-3) } \\
\text { Sturgeon River }\end{array}$ & $05-1300 \quad 00$ & $\begin{array}{ll}47 & 39 \\
93 & 01\end{array}$ & 36 & 2,694 & - & - & b5,931 & - & $\underline{\mathrm{CR}}$ & 1936 & $\begin{array}{l}\text { Minnesota Depart- } \\
\text { ment of Conserv }\end{array}$ & - \\
\hline $\begin{array}{l}\text { Ten Mile Lake (No 9-31) } \\
\text { Pomme de Terre River }\end{array}$ & $05-293200$ & $\begin{array}{ll}46 & 07 \\
95 & 56\end{array}$ & 140 & 2,208 & - & - & $b 6,180$ & - & $\mathrm{CR}$ & 1938 & $\begin{array}{l}\text { Minnesota Depart- } \\
\text { ment of Conserv }\end{array}$ & - \\
\hline $\begin{array}{l}\text { Thief Lake (No 1l-6) } \\
\text { Thief River }\end{array}$ & $\begin{array}{r}5 P A \\
05-0754 \quad 00\end{array}$ & $\begin{array}{ll}48 & 29 \\
95 & 57\end{array}$ & 280 & 7,100 & 60,350 & 92,300 & $\mathrm{~b} 31,950$ & - & $\mathrm{CR}$ & 1930 & $\begin{array}{l}\text { Minnesota Depart- } \\
\text { ment of Conserv }\end{array}$ & - \\
\hline $\begin{array}{l}\text { Wabana Lake (No 14-33) } \\
\text { Wabana Lake Outlet }\end{array}$ & $05-212800$ & $\begin{array}{ll}47 & 25 \\
93 & 30\end{array}$ & 54 & 4,420 & o) & 17,945 & 17,945 & - & $\mathrm{CR}$ & 1940 & $\begin{array}{l}\text { Minnesota Depart- } \\
\text { ment of Conserv }\end{array}$ & - \\
\hline $\begin{array}{l}\text { Whiteface } \\
\text { WhIteface River }\end{array}$ & $\begin{array}{r}4 \mathrm{AE} \\
04-020500\end{array}$ & $\begin{array}{ll}47 & 17 \\
92 & 11\end{array}$ & 130 & 5,440 & 0 & 81,920 & 81,920 & 136 & $\mathrm{P}$ & 1923 & $\begin{array}{l}\text { Minnesota Power and } \\
\text { Light Co }\end{array}$ & - \\
\hline $\begin{array}{l}\text { Winnibigoshish Lake } \\
\text { Mississippi River }\end{array}$ & $05-201000$ & $\begin{array}{ll}47 & 26 \\
94 & 03\end{array}$ & 1,442 & 114,799 & - & - & 653,570 & b1 2 & FMNR & 1884 & Corps of Engineers & $\mathrm{PA}$ \\
\hline $\begin{array}{l}\text { Winton Pool } \\
\text { Kawishiwi River }\end{array}$ & 5N-126900 & $\begin{array}{ll}47 & 56 \\
91 & 46\end{array}$ & 1,200 & - & - & - & 8,320 & $<05$ & $\mathrm{P}$ & - & $\begin{array}{l}\text { Minnesota Power and } \\
\text { Light Co }\end{array}$ & - \\
\hline \multicolumn{13}{|l|}{ MISSISSIPPI } \\
\hline $\begin{array}{l}\text { Arkabutla } \\
\text { Coldwater River }\end{array}$ & $\begin{array}{r}7 P A \\
07-278000\end{array}$ & $\begin{array}{ll}34 & 45 \\
90 & 07\end{array}$ & 1,000 & 33,400 & - & 525,300 & 493,800 & 5 & $\mathrm{FR}$ & 1945 & Corps of Engineers & $\mathrm{PA}$ \\
\hline $\begin{array}{l}\text { Enid } \\
\text { Yocona River }\end{array}$ & $07-2745^{7 P B} 00$ & $\begin{array}{ll}34 & 09 \\
89 & 54\end{array}$ & 560 & 28,000 & - & 660,000 & 602,400 & 10 & $\mathrm{FR}$ & 1953 & Corps of Engineers & PA \\
\hline $\begin{array}{l}\text { Grenada } \\
\text { Yalobusha R1ver }\end{array}$ & $07-2845 \quad$ PPD & $\begin{array}{ll}33 & 49 \\
89 & 46\end{array}$ & 1,320 & 64,600 & - & $1,337,400$ & $1,251,700$ & 9 & FR & 1954 & Corps of Engineers & PA \\
\hline $\begin{array}{cl}\text { Ross } \mathrm{R} & \text { Barnett } \\
\text { Pearl } & \text { River }\end{array}$ & $\mid \begin{array}{r}2 \mathrm{ZC} \\
02-4856^{00}\end{array}$ & $\begin{array}{ll}32 & 24 \\
90 & 04\end{array} \mid$ & 2,970 & 31,000 & - & 310,000 & 60,000 & $<05$ & $\mathrm{MR}$ & 1962 & \begin{tabular}{|} 
Pearl River Valley \\
Water Supply Dist
\end{tabular} & PA \\
\hline
\end{tabular}




\begin{tabular}{|c|c|c|c|c|c|c|c|c|c|c|c|}
\hline $\begin{array}{l}\text { Sardis } \\
\text { Tallahatchie River } \\
\text { MISSOURI }\end{array}$ & $\mid \begin{array}{r}7 P B \\
07-2720 \quad 00\end{array}$ & $\begin{array}{ll}34 & 24 \\
89 & 47\end{array}$ & 1,545 & 58,500 & - & $1,569,900$ & $1,478,000$ & 9 & $\mathrm{FR}$ & 1940 & Corps of Engineers \\
\hline $\begin{array}{l}\text { Clearwater } \\
\text { Black River }\end{array}$ & $\begin{array}{r}7 \mathrm{HC} \\
07-062000\end{array}$ & $\begin{array}{ll}37 & 08 \\
90 & 47\end{array}$ & 898 & 10,400 & $\mathrm{~b} 21,920$ & 413,700 & b391, 800 & 59 & $\mathrm{FR}$ & 1948 & Corps of Eng1neers \\
\hline $\begin{array}{l}\text { Lake of the Ozarks (Bagnell Dam) } \\
\text { Osage R1ver }\end{array}$ & $\begin{array}{r}6 \mathrm{SH} \\
06-9255 \mathrm{OO}\end{array}$ & $\begin{array}{ll}38 & 12 \\
92 & 37\end{array}$ & 14,000 & 59,700 & 727,000 & $1,973,000$ & $1,246,000$ & 2 & PR & 1931 & Union Electric Co \\
\hline $\begin{array}{l}\text { Lake Taneycomo } \\
\text { Wh1te River }\end{array}$ & $-7 \mathrm{HA}$ & $\begin{array}{ll}36 & 40 \\
93 & 07\end{array}$ & 4,362 & 2,200 & 17,400 & 30,900 & 13,500 & 05 & PR & 1913 & $\begin{array}{l}\text { Emplre District } \\
\text { Electric Co }\end{array}$ \\
\hline $\begin{array}{l}\text { Pomme de Terre } \\
\text { Pomme de Terre River }\end{array}$ & $06-9213^{6 S G}$ & $\begin{array}{ll}37 & 54 \\
93 & 19\end{array}$ & 611 & 16,100 & 12,000 & 650,000 & $\mathrm{~b} 407,000$ & 99 & $\mathrm{FR}$ & 1960 & Corps of Engineers \\
\hline $\begin{array}{l}\text { Table Rock } \\
\text { Wh1te River }\end{array}$ & $07-0534$ PHA & $\begin{array}{ll}36 & 36 \\
93 & 19\end{array}$ & 4,020 & 53,500 & 0 & $3,462,000$ & $3,462,000$ & 74 & FPR & 1956 & Corps of Engineers \\
\hline $\begin{array}{l}\text { Wappapello } \\
\text { St Franc1s River } \\
\text { MONTANA }\end{array}$ & $07-0390 \quad 000$ & $\begin{array}{ll}36 & 56 \\
90 & 17\end{array}$ & 1,310 & 23,200 & 4,000 & 625,000 & 621,000 & 5 & $F R$ & 2941 & Corps of Engineers \\
\hline $\begin{array}{l}\text { Ackley Lake } \\
\text { Judith River } \mathfrak{c}\end{array}$ & $06-1105^{6 C C} 00$ & $\begin{array}{rr}46 & 58 \\
109 & 56\end{array}$ & - & 247 & 325 & 6,142 & 5,817 & - & I & 1938 & $\begin{array}{l}\text { Ackley Lake Water } \\
\text { Users Assoc }\end{array}$ \\
\hline $\begin{array}{l}\text { Ashley Lake } \\
\text { Ashley Creek }\end{array}$ & $\begin{array}{r}12 \mathrm{CE} \\
12-3666 \mathrm{OO}\end{array}$ & $\begin{array}{rr}48 & 11 \\
114 & 37\end{array}$ & - & 3,000 & - & - & 20,000 & - & I & 1897 & $\begin{array}{l}\text { Ashley Irrigation } \\
\text { D1strict }\end{array}$ \\
\hline $\begin{array}{l}\text { Birch Creek (Swift Dam) } \\
\text { Birch Creek }\end{array}$ & $\begin{array}{r}6 \mathrm{BR} \\
06-094000\end{array}$ & $\begin{array}{rr}48 & 10 \\
112 & 52\end{array}$ & 753 & a455 & 18 & 30,005 & 29,985 & 3 & I & 1915 & $\begin{array}{l}\text { valier Land and } \\
\text { Water } c \circ\end{array}$ \\
\hline $\begin{array}{l}\text { Bynum } \\
\text { Teton R1ver } \subseteq /\end{array}$ & 06-1026 60 & $\begin{array}{rr}47 & 58 \\
112 & 25\end{array}$ & - & 4,120 & 500 & 75,000 & 74,500 & - & I & 1910 & $\begin{array}{l}\text { Teton Co-op Reser- } \\
\text { volr Co }\end{array}$ \\
\hline $\begin{array}{l}\text { Canyon Ferry } \\
\text { MIssouri River }\end{array}$ & $06-058500$ & $\begin{array}{rr}46 & 39 \\
111 & 44\end{array}$ & 15,904 & 35,200 & 8,000 & $2,051,000$ & $2,043,000$ & 6 & FIMPR & 1953 & $\begin{array}{l}\text { Bureau of } \\
\text { Reclamation }\end{array}$ \\
\hline $\begin{array}{l}\text { Como Lake } \\
\text { Rock Creek }\end{array}$ & $12-344500$ & $\begin{array}{rr}46 & 04 \\
114 & 14\end{array}$ & 546 & 936 & 0 & 34,890 & 34,890 & 3 & IR & 1909 & $\begin{array}{l}\text { B1tterroot Irriga- } \\
\text { t1on District }\end{array}$ \\
\hline $\begin{array}{l}\text { Cooney } \\
\text { Red Lodge Creek }\end{array}$ & $06-2120 \mathrm{FFH}$ & $\begin{array}{rr}45 & 27 \\
109 & 12\end{array}$ & 206 & 1,025 & 120 & $\mathrm{~d} 27,515$ & $\mathrm{~d} 27,395$ & 4 & I & 1936 & $\begin{array}{l}\text { Rock Creek Water } \\
\text { Users Assoc }\end{array}$ \\
\hline $\begin{array}{l}\text { Deadmans Basin } \\
\text { Musse1shell River } c \text { f }\end{array}$ & $06-122500$ & $\begin{array}{rr}46 & 21 \\
109 & 25\end{array}$ & - & 2,042 & 4,600 & 76,820 & 72,220 & - & I & 1941 & $\begin{array}{l}\text { Deadmans Basin } \\
\text { Water Users Assoc }\end{array}$ \\
\hline $\begin{array}{l}\text { Delmoe Park } \\
\text { Pipestone Creek }\end{array}$ & 06-0278 ${ }^{6 \mathrm{AH}}$ & \begin{tabular}{rr|}
45 & 59 \\
112 & 21
\end{tabular} & 36 & 479 & 0 & 6,600 & 6,600 & - & I & 1913 & $\begin{array}{l}\text { P1pestone Reservolr } \\
\text { and CanaI Co }\end{array}$ \\
\hline $\begin{array}{l}\text { Durand } \\
\text { North Fork Musselshell River }\end{array}$ & 06-1165 60 & $\begin{array}{rr}46 & 35 \\
110 & 34\end{array}$ & 486 & 272 & 24 & 7,029 & 7,005 & 8 & I & 1939 & $\begin{array}{l}\text { Upper Musselshell } \\
\text { Water Users Assoc }\end{array}$ \\
\hline
\end{tabular}


Table 1 --Reservoirs in the United States completed or under construction as of January 1, 1963, having a usable capac1ty of 5,000 acre-feet or more--Cont1nued [For meaning of symbols under "Use" and "Storage records published," see text]

\begin{tabular}{|c|c|c|c|c|c|c|c|c|c|c|c|c|}
\hline \multirow[b]{2}{*}{$\begin{array}{l}\text { Name of reservolr } \\
\text { and } \\
\text { stream }\end{array}$} & \multicolumn{2}{|c|}{ Location } & \multirow{2}{*}{$\begin{array}{l}\text { Drainage } \\
\text { area } \\
\text { (square } \\
\text { miles) }\end{array}$} & \multirow[b]{2}{*}{$\begin{array}{c}\text { Surface } \\
\text { area } \\
\text { (acres) }\end{array}$} & \multicolumn{3}{|c|}{ Storage in acre-feet } & \multirow[b]{2}{*}{$\begin{array}{c}\text { storage } \\
\text { rat10 }\end{array}$} & \multirow[b]{2}{*}{ Use } & \multirow[b]{2}{*}{$\begin{array}{c}\text { Date } \\
\text { com- } \\
\text { pleted }\end{array}$} & \multirow[b]{2}{*}{$\begin{array}{l}\text { Owner } \\
\text { or } \\
\text { operator }\end{array}$} & \multirow{2}{*}{$\begin{array}{l}\text { Storage } \\
\text { records } \\
\text { pub- } \\
\text { lished }\end{array}$} \\
\hline & \begin{tabular}{|c|} 
Basin \\
1ndex and \\
station no \\
\end{tabular} & \begin{tabular}{|l|}
$\begin{array}{l}\text { Lat1 tude } \\
\text { and } \\
\text { long1 tude }\end{array}$ \\
\end{tabular} & & & Dead & Total & Usable & & & & & \\
\hline MONTANA--Cont1nued & & & & & & & & & & & & \\
\hline $\begin{array}{l}\text { East Fork Rock Creek (Flint Creek } \\
\text { Dam) } \\
\text { East Fork Rock Creek }\end{array}$ & $\begin{array}{r}12 B D \\
12-332500\end{array}$ & $\begin{array}{rr}46 & 08 \\
113 & 23\end{array}$ & 303 & 442 & - & - & 16,040 & 08 & IR & 1937 & $\begin{array}{l}\text { Flint Creek Water } \\
\text { Users Assoc }\end{array}$ & $\mathrm{PA}$ \\
\hline $\begin{array}{l}\text { Ennis Lake } \\
\text { Madison River }\end{array}$ & $06-0405^{6 \mathrm{AL}} \mathrm{00}$ & $\begin{array}{rr}45 & 28 \\
211 & 38\end{array}$ & 2,181 & 3,800 & 1,040 & 42,060 & 41,020 & $<05$ & $\mathrm{PR}$ & 1900 & Montana Power Co & $P A$ \\
\hline $\begin{array}{l}\text { Flathead Lake (Kerr Dam) } \\
\text { Flathead RIver }\end{array}$ & $12-371000$ & $\begin{array}{rr}48 & 04 \\
114 & 14\end{array}$ & 7,086 & 126,000 & - & - & $1,219,000$ & 2 & FIPR & 1938 & Montana Power Co & PA \\
\hline $\begin{array}{l}\text { Fort Peck } \\
\text { Missour1 River }\end{array}$ & $\begin{array}{r}6 \mathrm{CK} \\
06-131500\end{array}$ & $\begin{array}{rr}48 & 00 \\
106 & 24\end{array}$ & 57,500 & 245,000 & 617,000 & $19,410,000$ & b13, 915, 000 & 20 & FINPR & 1939 & Corps of Engineers & PA \\
\hline $\begin{array}{l}\text { Four Horns } \\
\text { Badger Creek } \mathrm{c}\end{array}$ & $06-0930 \quad 00$ & $\begin{array}{rr}48 & 21 \\
112 & 42\end{array}$ & - & 897 & - & - & 19,250 & - & I & 1932 & $\begin{array}{l}\text { Bureau of Indian } \\
\text { Affairs }\end{array}$ & PA \\
\hline $\begin{array}{l}\text { Frenchman } \\
\text { Frenchman River }\end{array}$ & $06-164300$ & $\begin{array}{rr}48 & 42 \\
107 & 14\end{array}$ & a2,200 & 806 & 0 & 7,010 & 7,010 & - & I & 1952 & $\begin{array}{c}\text { Montana State Water } \\
\text { Conserv Board }\end{array}$ & PA \\
\hline $\begin{array}{l}\text { Fresno } \\
\text { Milk River }\end{array}$ & 06-1365 00 & $\begin{array}{rr}48 & 36 \\
109 & 57\end{array}$ & 3,766 & 5,757 & 1,860 & 129,100 & 127,200 & 5 & IR & 1939 & $\begin{array}{l}\text { Bureau of } \\
\text { Reclamation }\end{array}$ & PA \\
\hline $\begin{array}{l}\text { Georgetown Lake } \\
\text { Flint Creek }\end{array}$ & $12-325000$ & $\begin{array}{rr}46 & 13 \\
113 & 17\end{array}$ & 501 & 3,000 & - & - & 31,040 & - & PRW & 1905 & Montana Power Co & PA \\
\hline $\begin{array}{l}\text { Gibson } \\
\text { Sun River }\end{array}$ & $06-0795 \quad 6 \mathrm{BK}$ & $\begin{array}{rr}47 & 36 \\
112 & 46\end{array}$ & 575 & 1,360 & 0 & 105,000 & 105,000 & 2 & IR & 1929 & $\begin{array}{l}\text { Greenfields Irriga- } \\
\text { t1on District }\end{array}$ & PA \\
\hline $\begin{array}{l}\text { Hauser Lake } \\
\text { MIssouri River }\end{array}$ & $\begin{array}{r}6 \mathrm{BC} \\
06-0650 \quad 00\end{array}$ & $\begin{array}{rr}46 & 46 \\
111 & 54\end{array}$ & 16,876 & 3,800 & 46,810 & 98,230 & $\mathrm{~b} 42,550$ & $<05$ & PR & 1911 & Montana Power Co & PA \\
\hline $\begin{array}{l}\text { Hebgen } \\
\text { Madison River }\end{array}$ & $06-0380 \quad 00$ & $\begin{array}{rr}44 & 52 \\
111 & 20\end{array}$ & 904 & 12,668 & 7,340 & 384,800 & $\mathrm{~d} 377,500$ & 6 & PR & 1915 & Montana Power Co & PA \\
\hline $\begin{array}{l}\text { Holter } \\
\text { Missouri River }\end{array}$ & $06-0660 \quad 00$ & $\begin{array}{rr}46 & 59 \\
112 & 01\end{array}$ & 17,149 & 4,800 & 158,500 & 240,400 & $\mathrm{~b} 65,200$ & - & PR & 1918 & Montana Power Co & PA \\
\hline $\begin{array}{l}\text { Hubbart } \\
\text { Little B1tterroot River }\end{array}$ & $12-373500$ & $\begin{array}{rr}47 & 56 \\
114 & 44\end{array}$ & 114 & 460 & of & 12,100 & 12,100 & - & I & 1923 & $\begin{array}{l}\text { Bureau of Indian } \\
\text { Affalrs }\end{array}$ & PA \\
\hline $\begin{array}{l}\text { Hungry Horse } \\
\text { South Fork Flathead River }\end{array}$ & $\begin{array}{r}12 \mathrm{CD} \\
12-362000\end{array}$ & $\begin{array}{rr}48 & 20 \\
114 & 01\end{array}$ & 1,654 & 23,750 & 40,140 & $3,468,000$ & b2, 982,000 & 15 & FIP & 1952 & $\begin{array}{l}\text { Bureau of } \\
\text { Reclamation }\end{array}$ & PA \\
\hline $\begin{array}{l}\text { Kicking Horse } \\
\text { Crow Creek c/ }\end{array}$ & $\begin{array}{r}12 \mathrm{CH} \\
12-3797 \mathrm{OO}\end{array}$ & $\begin{array}{rr}47 & 27 \\
114 & 05\end{array}$ & - & 800 & 70 & 8,420 & 8,350 & - & I & 1930 & $\begin{array}{l}\text { Bureau of Indian } \\
\text { Affalrs }\end{array}$ & PA \\
\hline
\end{tabular}


Lake Adam

Grass Creek

Lake Frances

Dupuyer and Brich Creeks c/

Lake Helena

Missouri River

Lake Walvoord
Sweet Grass Creek $c /$

Lima
Red Rock River

Little Bitterroot Lake

Lit Biererroot River

Lower Crow

reek

Lower Jocko Lake

Miver

Lower Two Medicine Lake

Two Medicine Lake

MoDonald

Martinsdale

South Fork Musselshell River of

Middle Creek

thalt te Creek

Mission

Mission Creek

Morony

Missouri River

Mystic Lake
West Rosebud Creek

Nelson
M1lk River of

Nevada Creek

Nevada Creek

Nilan

Smith and Ford Creeks c/

Ninepipe
Flathead River / $/$

See footnotes at end of table

\begin{tabular}{|c|c|c|c|}
\hline $06-2007 \stackrel{6 F D}{00}$ & \begin{tabular}{rr|}
46 & 02 \\
109 & 50
\end{tabular} & - & 585 \\
\hline $\begin{array}{r}6 B R \\
06-095500\end{array}$ & $\begin{array}{rr}48 & 16 \\
112 & 12\end{array}$ & - & 5,536 \\
\hline $06-0645 \quad 00$ & $\left.\begin{array}{rr}46 & 46 \\
111 & 54\end{array}\right\}$ & 610 & 2,100 \\
\hline $06-2007 \quad 6 \mathrm{FD}$ & $\begin{array}{rr}46 & 00 \\
109 & 50\end{array}$ & - & 768 \\
\hline $\begin{array}{r}6 \mathrm{AA} \\
06-0120 \quad 00\end{array}$ & $\begin{array}{rr}44 & 39 \\
112 & 22\end{array}$ & 570 & $a 6,400$ \\
\hline $\begin{array}{r}12 \mathrm{CG} \\
12-372500\end{array}$ & $\begin{array}{rr}48 & 06 \\
114 & 42\end{array}$ & 318 & 2,994 \\
\hline $\begin{array}{r}12 \mathrm{CH} \\
12-3767 \text { OO }\end{array}$ & $\begin{array}{rr}47 & 30 \\
114 & 14\end{array}$ & - & 344 \\
\hline $\begin{array}{r}12 \mathrm{CH} \\
12-3805 \mathrm{OO}\end{array}$ & $\begin{array}{rr}47 & 12 \\
113 & 46\end{array}$ & 74 & 124 \\
\hline $\begin{array}{r}6 \mathrm{BP} \\
06-0909 \text { 00 }\end{array}$ & $\begin{array}{rr}48 & 30 \\
113 & 16\end{array}$ & 502 & 806 \\
\hline $\begin{array}{r}12 \mathrm{CH} \\
12-378200\end{array}$ & \begin{tabular}{rr|}
47 & 26 \\
114 & 00
\end{tabular} & 174 & 200 \\
\hline $\begin{array}{r}6 C F \\
06-1190 \text { OD }\end{array}$ & $\begin{array}{rr}46 & 27 \\
110 & 16\end{array}$ & - & 985 \\
\hline $\begin{array}{r}6 \mathrm{AP} \\
06-0495 \mathrm{OD}\end{array}$ & \begin{tabular}{rr|}
45 & 29 \\
110 & 59
\end{tabular} & 274 & 223 \\
\hline $\begin{array}{r}12 \mathrm{CH} \\
12-3772 \text { OO }\end{array}$ & $\begin{array}{rr}47 & 19 \\
114 & 01\end{array}$ & - & 289 \\
\hline $06-090200$ & $\begin{array}{rr}47 & 36 \\
111 & 03\end{array}$ & 19,600 & 300 \\
\hline $06-2040^{6 \mathrm{FF}}$ & $\begin{array}{rr}45 & 14 \\
109 & 46\end{array}$ & 464 & 440 \\
\hline $06-1550^{6 \mathrm{DH}}$ & $\begin{array}{rr}48 & 32 \\
107 & 32\end{array}$ & - & 4,560 \\
\hline $\begin{array}{r}12 B F \\
12-336500\end{array}$ & $\begin{array}{rr}46 & 48 \\
112 & 49\end{array}$ & 145 & 376 \\
\hline $\begin{array}{r}6 \mathrm{BK} \\
06-083000\end{array}$ & \begin{tabular}{rr|}
47 & 29 \\
112 & 32
\end{tabular} & - & a 520 \\
\hline $\begin{array}{r}12 \mathrm{CH} \\
12-380000\end{array}$ & $\begin{array}{rr}47 & 28 \\
114 & 08\end{array}$ & - & 1,580 \\
\hline
\end{tabular}

\begin{tabular}{|c|c|c|c|c|}
\hline 11,000 & - & I & 1912 & Big Timber Land Co \\
\hline 112,000 & - & IR & 1927 & $\begin{array}{l}\text { Valler Land and } \\
\text { Water Co }\end{array}$ \\
\hline 10,450 & - & CPR & 1945 & - \\
\hline 14,000 & - & I & 1912 & B1g Timber Land Co \\
\hline 84,050 & 8 & I & 1909 & $\begin{array}{l}\text { Water Users Irriga- } \\
\text { t1on Co }\end{array}$ \\
\hline 26,400 & - & IR & 1918 & $\begin{array}{l}\text { Bureau of Indian } \\
\text { Affairs }\end{array}$ \\
\hline 10,350 & 3 & IR & 1933 & $\begin{array}{l}\text { Bureau of Indian } \\
\text { Affairs }\end{array}$ \\
\hline 6,380 & 3 & IR & 1937 & $\begin{array}{l}\text { Eureau of Indian } \\
\text { Affairs }\end{array}$ \\
\hline 16,620 & 2 & IR & 1913 & $\begin{array}{l}\text { Bureau of Indian } \\
\text { Affatrs }\end{array}$ \\
\hline 8,220 & 2 & I & 1920 & $\begin{array}{l}\text { Bureau of Indian } \\
\text { Affairs }\end{array}$ \\
\hline 23,100 & - & I & 1939 & $\begin{array}{l}\text { Upper Musseishell } \\
\text { Water Users Assoc }\end{array}$ \\
\hline 8,027 & 3 & IMR & 1950 & $\begin{array}{l}\text { Montana State Water } \\
\text { Conserv Board }\end{array}$ \\
\hline 7,250 & 2 & I & 1935 & $\begin{array}{l}\text { Bureau of Indian } \\
\text { Affa1rs }\end{array}$ \\
\hline 7,900 & - & P & 1930 & Montana Power co \\
\hline $\mathrm{d} 20,800$ & - & $\mathrm{PR}$ & 1925 & Montana Power Co \\
\hline 66,800 & - & I & 1922 & $\begin{array}{l}\text { Bureau of } \\
\text { Reclamation }\end{array}$ \\
\hline 12,640 & 5 & FIR & 1938 & $\begin{array}{l}\text { Nevada Creek Water } \\
\text { Users Assoc }\end{array}$ \\
\hline 10,092 & - & I & 1951 & $\begin{array}{l}\text { Montana State Con- } \\
\text { servation Board }\end{array}$ \\
\hline 14,870 & - & CI & 1923 & $\begin{array}{l}\text { Bureau of Indian } \\
\text { Affairs }\end{array}$ \\
\hline
\end{tabular}



[For meaning of symbols under "Use" and "Storage records published," see text]

\begin{tabular}{|c|c|c|c|c|c|c|c|c|c|c|c|c|}
\hline \multirow[b]{2}{*}{$\begin{array}{l}\text { Name of reservo1r } \\
\text { and } \\
\text { stream }\end{array}$} & \multicolumn{2}{|c|}{ Location } & \multirow{2}{*}{$\begin{array}{l}\text { Drainage } \\
\text { area } \\
\text { (square } \\
\text { miles) }\end{array}$} & \multirow[b]{2}{*}{$\begin{array}{c}\text { Surface } \\
\text { area } \\
\text { (acres) }\end{array}$} & \multicolumn{3}{|c|}{ Storage in acre-feet } & \multirow[b]{2}{*}{$\begin{array}{c}\text { Storage } \\
\text { rat10 }\end{array}$} & \multirow[b]{2}{*}{ Use } & \multirow[b]{2}{*}{$\begin{array}{c}\text { Date } \\
\text { com- } \\
\text { pleted }\end{array} \mid$} & \multirow[b]{2}{*}{$\begin{array}{l}\text { Owner } \\
\text { or } \\
\text { operator }\end{array}$} & \multirow{2}{*}{$\begin{array}{l}\text { Storage } \\
\text { records } \\
\text { pub- } \\
\text { lished }\end{array}$} \\
\hline & $\begin{array}{c}\text { Basin } \\
\text { 1ndex and } \\
\text { station no } \\
\end{array}$ & $\begin{array}{c}\text { Lat1 tude } \\
\text { and } \\
\text { long1 tude }\end{array}$ & & & Dead & Total & Usable & & & & & \\
\hline MONTANA--Cont1nued & & & & & & & & & & & & \\
\hline $\begin{array}{l}\text { Noxon Rapids } \\
\text { Clark Fork }\end{array}$ & $12-391300$ & $\begin{array}{rr}47 & 58 \\
115 & 44\end{array}$ & 21,833 & 7,930 & - & 495,600 & 334,600 & $<0 \quad 05$ & $\mathrm{PR}$ & 1959 & $\begin{array}{l}\text { Washington Water } \\
\text { Power Co }\end{array}$ & PA \\
\hline $\begin{array}{l}\text { Pablo } \\
\text { Flathead Creek } c /\end{array}$ & $12-375900$ & $\begin{array}{rr}47 & 38 \\
114 & 08\end{array}$ & - & 1,746 & 0 & 27,100 & 27,100 & - & CI & 1934 & $\begin{array}{l}\text { Bureau of Indian } \\
\text { Affasirs }\end{array}$ & PA \\
\hline $\begin{array}{l}\text { Petrol1a } \\
\text { Flat W1llow and Elk Creeks }\end{array}$ & $06-1294 \quad 00$ & $\begin{array}{rr}46 & 48 \\
108 & 14\end{array}$ & - & 515 & 370 & 9,192 & 8,822 & 5 & I & 1951 & $\begin{array}{l}\text { Montana Water Con- } \\
\text { servation Board }\end{array}$ & PA \\
\hline $\begin{array}{l}\text { Pishkun } \\
\text { North Fork Sun River } c \text { / }\end{array}$ & $06-0805$ 00 & $\begin{array}{rr}47 & 41 \\
112 & 30\end{array}$ & - & 1,550 & 16,400 & 48,450 & 32,050 & - & I & 1925 & $\begin{array}{l}\text { Greenfields Irriga- } \\
\text { rion District }\end{array}$ & $\mathrm{PA}$ \\
\hline $\begin{array}{l}\text { Ruby River } \\
\text { Ruby River }\end{array}$ & $06-0205$ 6AD & $\begin{array}{rr}45 & 14 \\
212 & 06\end{array}$ & 596 & 970 & 100 & 38,950 & 38,850 & 3 & I & 1938 & $\begin{array}{l}\text { Ruby River Water } \\
\text { Users Assoc }\end{array}$ & $\mathrm{PA}$ \\
\hline $\begin{array}{l}\text { Sherburne Lake } \\
\text { Swiftcurrent Creek }\end{array}$ & $\mid \begin{array}{rr}5 S \\
05-0155 & 00\end{array}$ & $\begin{array}{rr}48 & 50 \\
113 & 31\end{array}$ & 637 & 1,730 & 0 & 66,200 & 66,200 & 4 & I & 1921 & $\begin{array}{l}\text { Bureau of } \\
\text { Reclamation }\end{array}$ & $\mathrm{PA}$ \\
\hline $\begin{array}{l}\text { Smith River (White Sulphur Dam) } \\
\text { Smith River }\end{array}$ & \begin{tabular}{rr|}
$6 \mathrm{BG}$ \\
$06-0750$ & 00
\end{tabular} & $\begin{array}{rr}46 & 37 \\
118 & 45\end{array}$ & 723 & 327 & 52 & 10,750 & 10,700 & - & IR & 1936 & $\begin{array}{l}\text { Smith River Water } \\
\text { Users Assoc }\end{array}$ & PA \\
\hline $\begin{array}{l}\text { Tabor } \\
\text { Dry Creek } \underline{c}\end{array}$ & $\begin{array}{r}12 \mathrm{CH} \\
12-377300\end{array}$ & $\begin{array}{rr}47 & 16 \\
113 & 56\end{array}$ & - & 286 & 0 & 23,300 & 23,300 & - & IR & 1919 & $\begin{array}{l}\text { Bureau of Indian } \\
\text { Affalrs }\end{array}$ & PA \\
\hline $\begin{array}{l}\text { Thompson Falls } \\
\text { Clark Fork }\end{array}$ & $12-390000$ & $\begin{array}{rr}47 & 35 \\
115 & 21\end{array}$ & 20,968 & 1,450 & - & - & 14,970 & $<05$ & $\mathrm{PR}$ & 1916 & Montana Power Co & $\mathrm{PA}$ \\
\hline $\begin{array}{l}\text { Tiber } \\
\text { Marias River }\end{array}$ & $06-1013 \quad 0 \mathrm{BU}$ & $\begin{array}{rr}48 & 19 \\
111 & 06\end{array}$ & 4,923 & 22,180 & 24,000 & $1,337,000$ & b762,000 & 12 & FIR & 1956 & $\begin{array}{l}\text { Bureau of } \\
\text { Reclamation }\end{array}$ & PA \\
\hline $\begin{array}{l}\text { Tongue River } \\
\text { Tongue River }\end{array}$ & $\begin{array}{r}6 \mathrm{GD} \\
06-3070 \quad 00\end{array}$ & $\begin{array}{rr}45 & 08 \\
106 & 46\end{array}$ & 1,770 & 3,497 & 1,400 & 69,439 & 68,039 & 2 & I & 1939 & $\begin{array}{l}\text { Tongue River water } \\
\text { Users Assoc }\end{array}$ & PA \\
\hline $\begin{array}{l}\text { Warhorse Lake } \\
\text { Buffalo Creek } \mathrm{c}\end{array}$ & $06-12769 \mathrm{CJ}$ & $\begin{array}{rr}47 & 07 \\
108 & 32\end{array}$ & - & - & 0 & 23,800 & 23,800 & - & I & 1916 & Winnett Irrigation & - \\
\hline $\begin{array}{l}\text { West Fork Bitterroot } \\
\text { West Fork Bitterroot River }\end{array}$ & $\begin{array}{r}12 \mathrm{BH} \\
12-342000\end{array}$ & $\begin{array}{rr}45 & 43 \\
114 & 17\end{array}$ & 317 & 655 & 656 & 32,362 & 31,706 & 2 & IR & 1940 & $\begin{array}{l}\text { West Fork Water } \\
\text { Users Assoc }\end{array}$ & PA \\
\hline $\begin{array}{l}\text { W1llow Creek } \\
\text { Lodgegrass Creek } \mathrm{c}\end{array}$ & $\begin{array}{r}6 \mathrm{HN} \\
06-291900\end{array}$ & $\begin{array}{rr}45 & 10 \\
107 & 36\end{array}$ & - & 750 & 0 & 23,000 & 23,000 & - & I & 1942 & $\begin{array}{l}\text { Bureau of Indian } \\
\text { Affairs }\end{array}$ & - \\
\hline $\begin{array}{l}\text { Willow Creek } \\
\text { Sun River } c \text { / }\end{array}$ & 06-0820 00 & $\begin{array}{rr}47 & 33 \\
112 & 25\end{array}$ & - & 1,450 & 67 & 32,400 & 32,330 & - & I & 1911 & $\begin{array}{l}\text { Greenfields Irriga- } \\
\text { tion Distifict }\end{array}$ & PA \\
\hline $\begin{array}{l}\text { Willow Creek } \\
\text { Willow Creek }\end{array}$ & $\left|\begin{array}{r}6 \mathrm{AH} \\
06-036000\end{array}\right|$ & $\begin{array}{rr}45 & 43 \\
111 & 42\end{array} \mid$ & 153 & 850 & 269 & 18,000 & 17,700 & 5 & IR & 1938 & $\begin{array}{l}\text { Willow Creek Water } \\
\text { Users Assoc }\end{array}$ & $\mathrm{PA}$ \\
\hline
\end{tabular}




\begin{tabular}{|c|}
\hline NEBRASKA \\
\hline $\begin{array}{l}\text { Box Butte } \\
\text { N1obrara R1ver }\end{array}$ \\
\hline $\begin{array}{l}\text { Enders } \\
\text { Frenchman Creek }\end{array}$ \\
\hline $\begin{array}{l}\text { Harlan County } \\
\text { Republican River }\end{array}$ \\
\hline $\begin{array}{l}\text { Harry Strunk Lake } \\
\text { Medicine Creek }\end{array}$ \\
\hline $\begin{array}{l}\text { Hugh Butler Lake } \\
\text { Red Willow Creek }\end{array}$ \\
\hline $\begin{array}{l}\text { Jeffrey Canyon } \\
\text { Platte River } c /\end{array}$ \\
\hline $\begin{array}{l}\text { Johnson } \\
\quad \text { Platte River } c /\end{array}$ \\
\hline $\begin{array}{l}\text { Lake Alice (Upper and Lower) } \\
\text { North Platte River } c \text { f }\end{array}$ \\
\hline $\begin{array}{l}\text { Lake Babcock } \\
\text { Loup River } \mathrm{c} /\end{array}$ \\
\hline $\begin{array}{l}\text { Lake Maloney } \\
\text { North Platte and South Platte } \\
\text { Rivers } \mathrm{c} /\end{array}$ \\
\hline $\begin{array}{l}\text { Lake Minatare } \\
\text { North Platte River } c \text { f }\end{array}$ \\
\hline $\begin{array}{l}\text { Lake McConaughy (Kingsley) } \\
\text { North Platte River }\end{array}$ \\
\hline $\begin{array}{l}\text { Lewis \& Clark Lake (Gavins Point } \\
\text { Dam)* } \\
\text { Missouri River }\end{array}$ \\
\hline $\begin{array}{l}\text { Merritt } \\
\text { Snake River }\end{array}$ \\
\hline $\begin{array}{l}\text { O1liver } \\
\text { Lodgepole Creek }\end{array}$ \\
\hline $\begin{array}{l}\text { Sherman } \\
\text { Middle Loup River } c /\end{array}$ \\
\hline
\end{tabular}

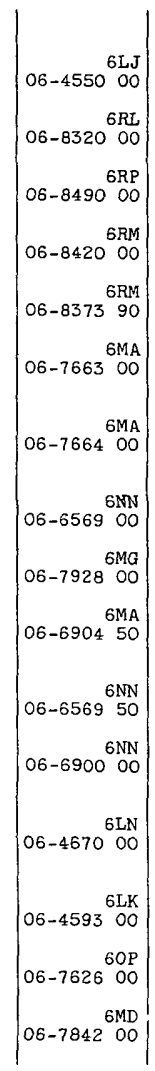

\begin{tabular}{|c|c|c|}
\hline $\begin{array}{r}42 \\
103 \\
104\end{array}$ & 1,500 & 1,600 \\
\hline $\begin{array}{rr}40 & 25 \\
101 & 31\end{array}$ & 1,300 & 2,560 \\
\hline $\begin{array}{ll}40 & 04 \\
99 & 12\end{array}$ & 20,753 & 22,800 \\
\hline $\begin{array}{r}4023 \\
100 \quad 13\end{array}$ & 850 & 4,820 \\
\hline $\begin{array}{r}4022 \\
100 \quad 40\end{array}$ & 600 & 2,682 \\
\hline $\begin{array}{rr}40 & 57 \\
100 & 24\end{array}$ & - & 620 \\
\hline $\begin{array}{ll}40 & 41 \\
99 & 50\end{array}$ & - & 2,420 \\
\hline $\begin{array}{r}4158 \\
103 \quad 34\end{array}$ & - & 1,684 \\
\hline $\begin{array}{ll}41 & 29 \\
97 & 21\end{array}$ & - & 900 \\
\hline $\begin{array}{rr}41 & 03 \\
100 & 46\end{array}$ & - & 1,670 \\
\hline $\begin{array}{r}4155 \\
10330\end{array}$ & - & 2,160 \\
\hline $\begin{array}{rr}41 & 13 \\
101 & 40\end{array}$ & 30,000 & 32,200 \\
\hline $\begin{array}{ll}42 & 51 \\
97 & 29\end{array}$ & 279,500 & 17,000 \\
\hline $\begin{array}{r}4238 \\
10052\end{array}$ & 620 & 2,906 \\
\hline $\begin{array}{rr}41 & 14 \\
103 & 48\end{array}$ & 1,380 & - \\
\hline $\begin{array}{ll}41 & 18 \\
98 & 52\end{array}$ & - & 2,845 \\
\hline
\end{tabular}

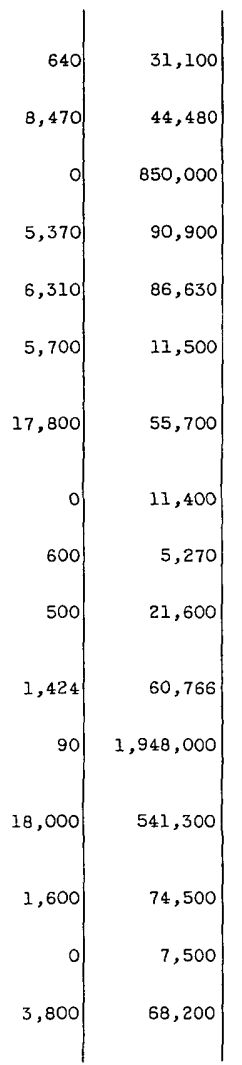

\begin{tabular}{|c|c|c|}
\hline 30,460 & 11 & IR \\
\hline 36,010 & 7 & FIR \\
\hline 850,000 & 16 & FI \\
\hline 33,860 & 5 & FI \\
\hline 31,470 & 9 & FI \\
\hline 5,800 & - & IP \\
\hline 37,900 & - & IP \\
\hline 11,400 & - & I \\
\hline 4,670 & - & $P$ \\
\hline 11,950 & - & IPR \\
\hline 59,342 & - & I \\
\hline$, 948,000$ & 18 & IP \\
\hline 523,000 & $<05$ & FINPR \\
\hline 72,900 & 4 & I \\
\hline 7,500 & 8 & I \\
\hline 64,400 & - & I \\
\hline
\end{tabular}

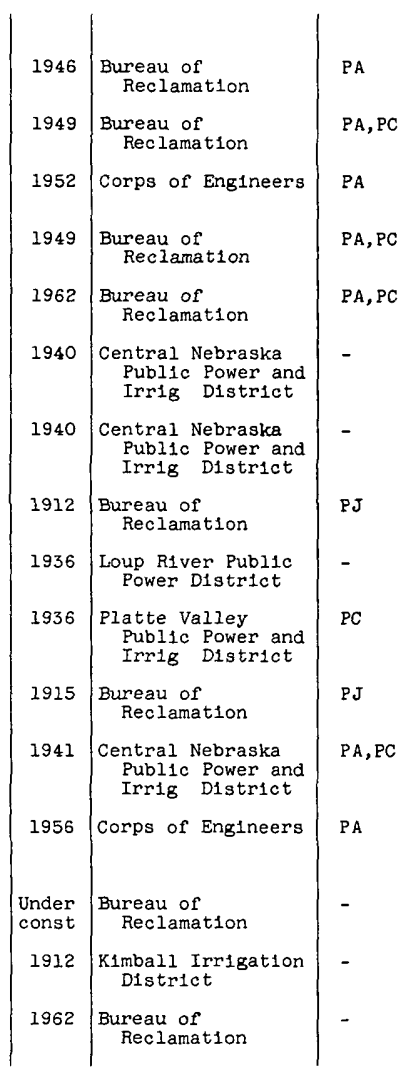


Table 1 --Reservo1rs in the Un1ted States completed or under construction as of January 1, 1963, having a usable capac1ty of 5,000 acre-feet or more--Continued [For meaning of symbols under "Use" and "Storage records publlshed," see text]

\begin{tabular}{|c|c|c|c|c|c|c|c|c|c|c|c|c|}
\hline \multirow[b]{2}{*}{$\begin{array}{l}\text { Name of reservoir } \\
\text { and } \\
\text { stream }\end{array}$} & \multicolumn{2}{|c|}{ Location } & \multirow{2}{*}{$\begin{array}{l}\text { Drainage } \\
\text { area } \\
\text { (square } \\
\text { m1les) }\end{array}$} & \multirow[b]{2}{*}{$\begin{array}{c}\text { Surface } \\
\text { area } \\
\text { (acres) }\end{array}$} & \multicolumn{3}{|c|}{ Storage in acre-feet } & \multirow[b]{2}{*}{ 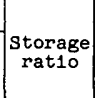 } & \multirow[b]{2}{*}{ Use } & \multirow[b]{2}{*}{$\begin{array}{c}\text { Date } \\
\text { com- } \\
\text { pleted }\end{array}$} & \multirow[b]{2}{*}{$\begin{array}{l}\text { Owner } \\
\text { or } \\
\text { operator }\end{array}$} & \multirow{2}{*}{$\begin{array}{l}\text { Storage } \\
\text { records } \\
\text { pub- } \\
\text { lished }\end{array}$} \\
\hline & \begin{tabular}{|c|} 
Basin \\
1ndex and \\
station no
\end{tabular} & $\begin{array}{l}\text { Lat1 tude } \\
\text { and } \\
\text { long1tude }\end{array}$ & & & Dead & Total & Usable & & & & & \\
\hline NEBRASKA--Cont Inued & & & & & & & & & & & & \\
\hline $\begin{array}{l}\text { Sutherland } \\
\text { North Platte and South Platte } \\
\text { RIvers, } \subseteq\end{array}$ & $06-6904 \quad 0 \mathrm{NN}$ & $\begin{array}{rr}41 & 06 \\
101 & 06\end{array}$ & - & 4,350 & 2,960 & 181,460 & 72,480 & - & IP & 1936 & $\begin{array}{l}\text { Platte Valley } \\
\text { Public Power and } \\
\text { Irrig District }\end{array}$ & PC \\
\hline $\begin{array}{l}\text { Swanson Lake (Trenton Dam) } \\
\text { Republican River }\end{array}$ & $\begin{array}{r}6 \mathrm{RM} \\
06-8290 \\
00\end{array}$ & $\begin{array}{rr}40 & 10 \\
101 & 04\end{array}$ & 8,100 & 10,040 & 4,200 & 254,000 & 249,900 & 19 & IF & 1953 & $\begin{array}{l}\text { Bureau of } \\
\text { Reclamation }\end{array}$ & $\mathrm{PA}, \mathrm{PC}$ \\
\hline $\begin{array}{l}\text { Whitney } \\
\text { White River of } \\
\text { NEVADA }\end{array}$ & $06-444800$ & $\begin{array}{rr}42 & 47 \\
103 & 17\end{array}$ & - & - & of & 10,000 & 10,000 & - & I & 1925 & $\begin{array}{l}\text { Whitney Irrigation } \\
\text { District }\end{array}$ & $\mathrm{PC}$ \\
\hline $\begin{array}{l}\text { Alkali Lake (Topaz Dam) } \\
\text { West Walker Feeder Canal } \underline{\mathrm{c}}\end{array}$ & $10-297000$ & $\begin{array}{rr}38 & 41 \\
119 & 31\end{array}$ & - & 2,300 & - & - & 59,44 이 & - & IR & 1937 & $\begin{array}{l}\text { Walker River Irr1- } \\
\text { gation District }\end{array}$ & PA \\
\hline $\begin{array}{l}\text { B1shop Creek (Metropolis) } \\
\text { B1shop Creek }\end{array}$ & $-{ }^{1 O D A}$ & \begin{tabular}{rr|}
41 & 15 \\
114 & 55
\end{tabular} & - & - & 0 & 30,000 & 30,000 & - & I & 1912 & $\begin{array}{l}\text { Pac1fic Reclama- } \\
\text { tion Co }\end{array}$ & - \\
\hline $\begin{array}{l}\text { Lahontan } \\
\quad \text { Carson River } c /\end{array}$ & $\begin{array}{r}10 c C \\
10-312100\end{array}$ & $\begin{array}{rr}39 & 28 \\
119 & 04\end{array}$ & - & 10,000 & 0 & 273,600 & 273,600 & - & IP & 1915 & $\begin{array}{l}\text { Bureau of } \\
\text { Reclamation }\end{array}$ & PA \\
\hline $\begin{array}{l}\text { Lake Mead (Hoover Dam)* } \\
\text { Colorado RIver }\end{array}$ & $09-421000$ & $\begin{array}{rr}36 & 01 \\
114 & 44\end{array} \mid$ & 167,800 & 157,736 & $2,620,000$ & $29,827,000$ & $27,207,000$ & 22 & FIMNP & 1936 & $\begin{array}{l}\text { Bureau of } \\
\text { Reclamation }\end{array}$ & PA \\
\hline $\begin{array}{l}\text { Lake Mohave (Davis Dam)* } \\
\text { Colorado River }\end{array}$ & $09-422500$ & $\begin{array}{rr}35 & 12 \\
114 & 34\end{array}$ & 169,300 & 28,192 & 3,530 & $1,818,300$ & $1,809,800$ & - & IP & 1949 & $\begin{array}{l}\text { Bureau of } \\
\text { Reclamation }\end{array}$ & PA \\
\hline $\begin{array}{l}\text { Pitt-Taylor No } 1 \\
\text { Humboldt R1ver } \mathrm{c} /\end{array}$ & $-^{10 D K}$ & $\begin{array}{rr}40 & 36 \\
118 & 18\end{array}$ & - & - & of & 28,900 & 28,900 & - & I & 1914 & $\begin{array}{l}\text { Humboldt-Lovelock } \\
\text { Irrig 'L1ght and } \\
\text { Power co }\end{array}$ & - \\
\hline $\begin{array}{l}\text { P1tt-Taylor No }{ }^{2} \\
\quad \text { Humboldt River } \subseteq\end{array}$ & $-^{10 D K}$ & $\begin{array}{rr}a 40 & 36 \\
\text { all8 } & 18\end{array}$ & - & - & o & 20,800 & 20,800 & - & I & 1914 & $\begin{array}{l}\text { Humboldt-Lovelock } \\
\text { Irr1gation, L1ght } \\
\text { and Power Co }\end{array}$ & - \\
\hline $\begin{array}{l}\text { Rye Patch } \\
\text { Humboldt River }\end{array}$ & $\begin{array}{r}10 D K \\
10-334500\end{array}$ & \begin{tabular}{rr|}
40 & 28 \\
118 & 18
\end{tabular} & 13,700 & 10,820 & of & 179,100 & 179,100 & - & I & 1936 & $\begin{array}{l}\text { Pershing County } \\
\text { Conserv District }\end{array}$ & $\mathrm{PA}$ \\
\hline $\begin{array}{l}\text { Weber } \\
\text { Walker River }\end{array}$ & $-^{10 E C}$ & $\begin{array}{rr}39 & 04 \\
118 & 54\end{array}$ & a2,, 800 & 960 & o & 13,000 & 13,000 & - & I & 1935 & $\begin{array}{l}\text { Bureau of Indian } \\
\text { Affa1rs }\end{array}$ & - \\
\hline $\begin{array}{l}\text { W1ld Horse } \\
\text { Owyhee R1ver }\end{array}$ & $\begin{array}{r}13 D E \\
13-174000\end{array}$ & \begin{tabular}{rr|}
41 & 41 \\
115 & 51
\end{tabular} & 209 & 1,830 & of & 32,690 & 32,690 & 10 & I & 1937 & $\begin{array}{l}\text { Bureau of Indian } \\
\text { Affairs }\end{array}$ & PA \\
\hline $\begin{array}{l}\text { Willow Creek } \\
\text { W1llow Creek }\end{array}$ & $-10 D F$ & $\begin{array}{rr}41 & 15 \\
116 & 35\end{array}$ & - & 668 & o & 18,064 & 18,064 & - & I & 1924 & $\underbrace{\text { Ellison Ranching }}_{\text {Co }}$ & - \\
\hline
\end{tabular}


NEW HAMPSHIRE

Blackwater

Bow Lake

Isinglass River

Comerford Station Pond *

Connecticut River

Conway Lake
Saco River tributary

Edward Macdowell

Everett

Piscataquog River

First Connecticut Lake

Connecticut River

Franklin Falls

Pemigewasset River

Goose Pond

Goose Pond Brook

Great East Lake *

Salmon Falls River

Hopkinton
Contoocook River

Jackman
North Branch Contoocook River

Lake Francis (Murphy Dam)
Connecticut River

Lake Wentworth

Lake Winnipesaukee tributary

Lake Winnipesaukee
W1nnipesaukee R1ve

Mascoma Lake

Mascoma River

Massabes1c Lake

Cohas Brook

Merrymeeting Lake

See footnotes at end of table

\begin{tabular}{|c|c|c|c|c|}
\hline $\mid \begin{array}{r}1 E C \\
01-086500\end{array}$ & $\begin{array}{ll}43 & 19 \\
71 & 43\end{array}$ & 128 & 3,140 & - \\
\hline $\mid \begin{array}{r}1 D J \\
01-072750\end{array}$ & $\begin{array}{ll}43 & 15 \\
71 & 09\end{array}$ & 146 & 1,161 & - \\
\hline $\mid \begin{array}{r}1 \mathrm{~GB} \\
01-132500\end{array}$ & $\begin{array}{ll}44 & 20 \\
72 & 00\end{array}$ & 1,635 & 1,093 & 23,620 \\
\hline $\begin{array}{r}1 D E \\
01-0645 \quad 20\end{array}$ & $\begin{array}{ll}43 & 59 \\
71 & 03\end{array}$ & 30 & 1,730 & o \\
\hline $\begin{array}{r}\text { IED } \\
01-082500\end{array}$ & $\begin{array}{ll}42 & 54 \\
71 & 59\end{array}$ & 44 & 840 & - \\
\hline 1E1-0907 00 & $\begin{array}{ll}43 & 06 \\
71 & 40\end{array}$ & 63 & 2,800 & - \\
\hline $\begin{array}{r}1 \mathrm{GA} \\
01-128000\end{array}$ & $\begin{array}{ll}45 & 05 \\
71 & 17\end{array}$ & 830 & 3,125 & 0 \\
\hline $\begin{array}{r}1 E A \\
01-0785 \text { ON }\end{array}$ & $\begin{array}{ll}43 & 28 \\
71 & 40\end{array}$ & 1,000 & 2,840 & - \\
\hline $\begin{array}{r}1 \mathrm{GG} \\
01-148000\end{array}$ & $\begin{array}{ll}43 & 41 \\
72 & 06\end{array}$ & 157 & 668 & 0 \\
\hline 101-0700 100 & $\begin{array}{ll}43 & 35 \\
70 & 56\end{array}$ & 170 & 1,690 & 0 \\
\hline |01-0854 1 1ED & $\begin{array}{ll}43 & 12 \\
71 & 45\end{array}$ & - & 3,700 & - \\
\hline $\begin{array}{r}1 E D \\
01-084450\end{array}$ & $\begin{array}{ll}43 & 07 \\
71 & 57\end{array}$ & 69 & 519 & - \\
\hline $01-129000$ & $\begin{array}{ll}45 & 03 \\
71 & 23\end{array}$ & 170 & 2,010 & 0 \\
\hline $\begin{array}{r}\text { 1EB } \\
01-0795 \text { O0 }\end{array}$ & $\begin{array}{ll}43 & 35 \\
71 & 12\end{array}$ & 23 & 3,165 & - \\
\hline O1-0805 00 & $\begin{array}{ll}43 & 33 \\
71 & 28\end{array}$ & 363 & 44,800 & - \\
\hline $\begin{array}{r}1 G G \\
01-150000\end{array}$ & $\begin{array}{ll}43 & 39 \\
72 & 11\end{array}$ & 153 & 1,155 & 2,383 \\
\hline $\begin{array}{r}\text { IEF } \\
01-0935 \quad 00\end{array}$ & $\begin{array}{ll}42 & 58 \\
71 & 24\end{array}$ & 43 & 2,563 & - \\
\hline |01-0790 $\begin{array}{r}\text { 1EB } \\
00\end{array}$ & \begin{tabular}{ll|}
43 & 29 \\
71 & 11
\end{tabular} & 12 & 1,110 & - \\
\hline
\end{tabular}

\begin{tabular}{|c|c|c|}
\hline 46,000 & 3 & $\mathrm{~F}$ \\
\hline 11,500 & - & $\mathrm{R}$ \\
\hline 29,356 & $<05$ & $\mathrm{P}$ \\
\hline 11,500 & - & PR \\
\hline 12,800 & 2 & $F$ \\
\hline 86,500 & - & $\mathrm{FR}$ \\
\hline 76,493 & 5 & $\mathrm{P}$ \\
\hline 153,700 & 1 & $F$ \\
\hline 11,688 & 8 & P \\
\hline 11,800 & 9 & $P$ \\
\hline 70,800 & 3 & FR \\
\hline 8,400 & $\begin{array}{ll}- & -1 \\
-1\end{array}$ & P \\
\hline 99,309 & 4 & FPI \\
\hline 19,600 & - & PR \\
\hline 166,000 & 4 & $\mathrm{PR}$ \\
\hline 7,744 & 05 & $\mathrm{P}$ \\
\hline 16,600 & 4 & MP \\
\hline 8,450 & - & $\mathrm{R}$ \\
\hline
\end{tabular}

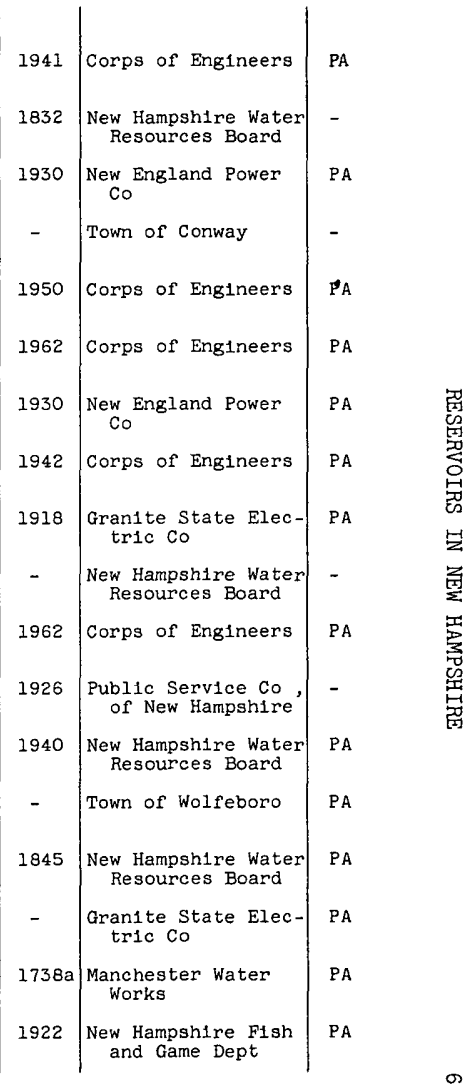


Table 1 --Reservoirs in the United States completed or under construction as of January 1, 1963, hav1ng a usable capac1ty of 5,000 acre-feet or more--Continued [For meaning of symbols under "Use" and "Storage records published," see text]

\begin{tabular}{|c|c|c|c|c|c|c|c|c|c|c|c|c|}
\hline \multirow[b]{2}{*}{$\begin{array}{l}\text { Name of reservolr } \\
\text { and } \\
\text { stream }\end{array}$} & \multicolumn{2}{|c|}{ Location } & \multirow{2}{*}{$\begin{array}{c}\text { Drainage } \\
\text { area } \\
\text { (square } \\
\text { m1les) }\end{array}$} & \multirow[b]{2}{*}{$\begin{array}{c}\text { Surface } \\
\text { area } \\
\text { (acres) }\end{array}$} & \multicolumn{3}{|c|}{ Storage in acre-feet } & \multirow[b]{2}{*}{$\mid \begin{array}{c}\text { Storage } \\
\text { rat1o }\end{array}$} & \multirow[b]{2}{*}{ Use } & \multirow[b]{2}{*}{$\begin{array}{c}\text { Date } \\
\text { com- } \\
\text { pleted }\end{array}$} & \multirow[b]{2}{*}{$\begin{array}{l}\text { Owner } \\
\text { or } \\
\text { operator }\end{array}$} & \multirow{2}{*}{$\begin{array}{l}\text { Storage } \\
\text { records } \\
\text { pub- } \\
\text { 11shed }\end{array}$} \\
\hline & $\begin{array}{l}\text { Basin } \\
\text { 1ndex and } \\
\text { station no }\end{array}$ & $\begin{array}{l}\text { Lat1 tude } \\
\text { and } \\
\text { long1 tude }\end{array}$ & & & Dead & Total & Usable & & & & & \\
\hline NEW HAMPSHIRE--Cont 1 nued & & & & & & & & & & & & \\
\hline $\begin{array}{l}\text { Littleton Development (Samuel C } \\
\text { Moore Dam)* } \\
\text { Connecticut River }\end{array}$ & $\begin{array}{r}1 G B \\
01-1320 \quad 00\end{array}$ & $\begin{array}{ll}44 & 20 \\
71 & 52\end{array}$ & 1,600 & 3,490 & 109,676 & 223,852 & 114,176 & 005 & $P$ & 1956 & $\begin{array}{l}\text { New England Power } \\
\text { Co }\end{array}$ & PA \\
\hline $\begin{array}{l}\text { Newfound Lake } \\
\text { Newfound River }\end{array}$ & 1E1-0775 $\quad 00$ & $\begin{array}{ll}43 & 37 \\
71 & 44\end{array}$ & 980 & 4,106 & - & - & 38,800 & - & PR & - & $\begin{array}{l}\text { Public Service Co } \\
\text { of New Hampshire }\end{array}$ & $\mathrm{PA}$ \\
\hline $\begin{array}{l}\text { North Weare } \\
\text { P1scataquog R1ver }\end{array}$ & $\begin{array}{r}\text { IED } \\
01-090650\end{array}$ & $\begin{array}{ll}43 & 07 \\
71 & 46\end{array}$ & 33 & 323 & - & - & 6,300 & - & $R$ & 1940 & $\begin{array}{l}\text { New Hampshire Water } \\
\text { Resources Board }\end{array}$ & - \\
\hline $\begin{array}{l}\text { Ossipee Lake } \\
\text { Ossipee River }\end{array}$ & 01-064990 & $\begin{array}{ll}43 & 48 \\
71 & 04\end{array}$ & 330 & 3,880 & - & - & 23,000 & $<05$ & PR & 1944 & $\begin{array}{c}\text { Central Maine Power } \\
\text { Co }\end{array}$ & - \\
\hline $\begin{array}{l}\text { Otter Brook } \\
\text { Otter Brook }\end{array}$ & $\begin{array}{r}1 G \mathrm{GK} \\
01-158500\end{array}$ & $\begin{array}{ll}42 & 57 \\
72 & 14\end{array}$ & $\begin{array}{ll}47 & 2\end{array}$ & 374 & - & - & 18,320 & 3 & $\mathrm{FR}$ & 1958 & Corps of Engineers & PA \\
\hline $\begin{array}{l}\text { Pawtuckaway Pond } \\
\text { Pawtuckaway River }\end{array}$ & $\mid$\begin{tabular}{rr|}
$1 D J$ \\
$01-0732$ & 50
\end{tabular} & $\begin{array}{ll}43 & 04 \\
71 & 09\end{array}$ & 207 & 902 & - & - & 8,100 & - & $P R$ & $1825 a$ & $\begin{array}{l}\text { New Hampshire Water } \\
\text { Resources Board }\end{array}$ & - \\
\hline $\begin{array}{l}\text { Pine River Pond } \\
\text { Pine River }\end{array}$ & $01-064970$ & $\begin{array}{ll}43 & 38 \\
71 & 03\end{array}$ & 13 & 600 & - & - & 6,500 & - & PRW & - & W M Lord Co & - \\
\hline $\begin{array}{l}\text { Second Connecticut Lake } \\
\text { Connecticut River }\end{array}$ & $01-127850$ & $\begin{array}{ll}45 & 08 \\
71 & 11\end{array}$ & $\begin{array}{ll}45 & 4\end{array}$ & 1,272 & 0 & 11,613 & 11,613 & 15 & $\mathrm{P}$ & 1934 & $\begin{array}{l}\text { New England Power } \\
\text { Co }\end{array}$ & $\mathrm{PA}$ \\
\hline $\begin{array}{l}\text { Squam and Little Squam Lakes } \\
\text { Squam River }\end{array}$ & $\mid 01-076950$ & $\begin{array}{ll}43 & 42 \\
71 & 38\end{array}$ & 576 & - & - & - & 37,900 & 6 & PRW & 1927 & Town of Ashland & - \\
\hline $\begin{array}{l}\text { Sunapee Lake } \\
\text { Sugar River }\end{array}$ & $\mid \begin{array}{r}1 G G \\
01-152000\end{array}$ & $\begin{array}{ll}43 & 23 \\
72 & 05\end{array}$ & $\begin{array}{ll}45 & 5\end{array}$ & 24,200 & - & - & 19,800 & 4 & MPR & 1928 & $\begin{array}{l}\text { New Hampshire Water } \\
\text { Resources Board }\end{array}$ & PA \\
\hline $\begin{array}{l}\text { Surry Mountain } \\
\text { Ashuelot River }\end{array}$ & $01-157500$ & $\begin{array}{ll}43 & 00 \\
72 & 19\end{array}$ & 100 & 980 & - & - & 32,600 & 3 & $\mathrm{FR}$ & 1942 & Corps of Engineers & PA \\
\hline $\begin{array}{l}\text { Three Ponds-Milton, Northeast, and } \\
\text { Townhouse * } \\
\text { Salmon Falls River }\end{array}$ & $01-0720 \quad 00$ & $\begin{array}{ll}43 & 25 \\
70 & 59\end{array}$ & 111 & - & 0 & 16,200 & 16,200 & 1 & $P$ & - & $\begin{array}{c}\text { New Hampshire Water } \\
\text { Resources Board }\end{array}$ & - \\
\hline $\begin{array}{l}\text { Umbagog Lake (Errol Dam) } \\
\text { Androscoggin River }\end{array}$ & $\mid \begin{array}{r}\text { IDA } \\
01-0530 \quad 00\end{array}$ & $\begin{array}{ll}44 & 47 \\
71 & 07\end{array}$ & 1,045 & 10,100 & of & 70,700 & 70,700 & 05 & LP & 1888 & $\begin{array}{l}\text { Union water Power } \\
\text { Co }\end{array}$ & $\mathrm{PA}$ \\
\hline $\begin{array}{l}\text { Winnisgam } \\
\text { Winnipesaukee River }\end{array}$ & $01-080900$ & $\begin{array}{ll}43 & 28 \\
71 & 32\end{array}$ & 430 & 4,264 & - & - & 38,000 & 08 & $\mathrm{PR}$ & 1910 & $\begin{array}{l}\text { Public Service Co } \\
\text { of New Hampshire }\end{array}$ & - \\
\hline
\end{tabular}


NEW JERSEY

\section{Boonton
Rockaway}

River

Cantstear
Pacock Brook

Charlotteburg

Pequannock River

c1inton

Pequannock RIver

Greenwood Lake
Wanaque River

Lake Hopatcong

Musconetcong River

Lake Mohawk

Wallkill River

Oak R1dge

Pequannock River

Oradeli

Hackensack RIver

Splitrock

Beaver Brook

Union Lake

Maurice R1ver

Wanaque

Wanaque River

\section{NEW MEXICO}

Abiquiu

Rio Cham

Al amogordo
Pecos River

Bluewater Lake

Bluewater Creek

Caballo

Grande

Conchas

Canadian River

Costilla Lake

See footnotes at end of table

\begin{tabular}{|c|c|c|c|}
\hline $01-3809^{1 \mathrm{KJ}} 00$ & $\begin{array}{ll}40 & 54 \\
74 & 24\end{array}$ & 119 & 780 \\
\hline $01-382100$ & $\begin{array}{ll}41 & 07 \\
74 & 30\end{array}$ & 56 & 350 \\
\hline $01-3823 \quad \begin{array}{l}1 \mathrm{KH} \\
50\end{array}$ & $\begin{array}{ll}41 & 02 \\
74 & 25\end{array}$ & $\begin{array}{ll}56 & 2\end{array}$ & - \\
\hline $01-3823^{1 \mathrm{KH}}$ & $\begin{array}{ll}41 & 04 \\
74 & 27\end{array}$ & 105 & 423 \\
\hline $01-3830 \stackrel{1 \mathrm{KH}}{00}$ & $\begin{array}{ll}41 & 10 \\
74 & 20\end{array}$ & $\begin{array}{ll}27 & 1\end{array}$ & 1,920 \\
\hline $\begin{array}{r}\text { IMF } \\
01-4554 \quad 00\end{array}$ & $\begin{array}{ll}40 & 55 \\
74 & 40\end{array}$ & 256 & 2,443 \\
\hline$-1 \mathrm{KC}$ & $\begin{array}{ll}41 & 02 \\
74 & 38\end{array}$ & $\begin{array}{ll}4 & 3\end{array}$ & 777 \\
\hline $01-3822^{1 \mathrm{KH}} 00$ & $\begin{array}{ll}41 & 02 \\
74 & 30\end{array}$ & $\begin{array}{ll}33 & 7\end{array}$ & 482 \\
\hline $\begin{array}{r}1 \mathrm{KG} \\
01-378480\end{array}$ & $\begin{array}{ll}40 & 57 \\
74 & 02\end{array}$ & 113 & 650 \\
\hline $01-3799^{1 \mathrm{KH}}$ & $\begin{array}{ll}40 & 58 \\
74 & 28\end{array}$ & 55 & 566 \\
\hline$-^{1 M E}$ & $\begin{array}{ll}39 & 24 \\
75 & 03\end{array}$ & 218 & 825 \\
\hline $01-386990$ & $\begin{array}{ll}41 & 02 \\
74 & 18\end{array}$ & 904 & 2,310 \\
\hline $08-2869$ OBJ & 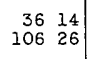 & 2,146 & 4,806 \\
\hline $08-3840^{8 \mathrm{FE}}$ & $\begin{array}{rr}34 & 36 \\
104 & 23\end{array}$ & 4,390 & 4,570 \\
\hline $\begin{array}{r}8 B P \\
08-3414 \quad 00\end{array}$ & $\begin{array}{rr}34 & 18 \\
108 & 07\end{array}$ & 201 & 1,746 \\
\hline $\begin{array}{r}8 \mathrm{CE} \\
08-3620^{\circ}\end{array}$ & $\begin{array}{rr}32 & 54 \\
107 & 17\end{array}$ & 30,700 & 11,613 \\
\hline $\begin{array}{r}\text { 7CE } \\
07-2235 \text { 00 }\end{array}$ & $\begin{array}{rr}35 & 24 \\
104 & 11\end{array}$ & 7,409 & 9,594 \\
\hline $\begin{array}{r}8 \mathrm{BF} \\
08-253900\end{array}$ & $\begin{array}{rr}36 & 53 \\
105 & 17\end{array}$ & 55 & 409 \\
\hline
\end{tabular}

26,000
7,400
8,900
10,800
43,200
47,800
7,100
12,000
8,750
-
7,440
85,960
344,000
370,200
15,700
122,100
38,500

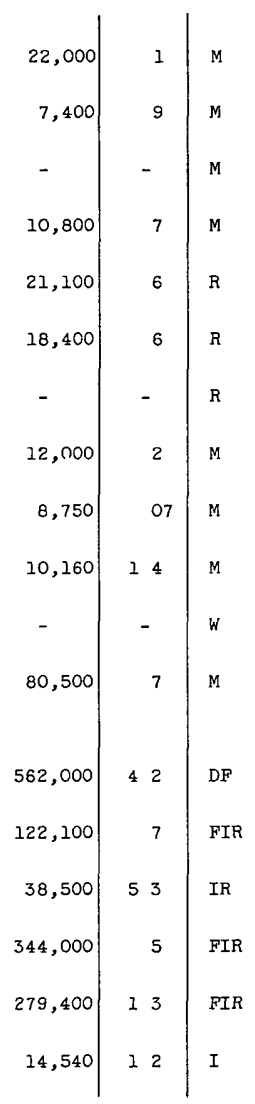

\begin{tabular}{|c|c|c|}
\hline 1904 & Jersey C1ty & PA \\
\hline- & City of Newark & $\mathrm{PA}, \mathrm{PN}$ \\
\hline 1961 & City of Newark & - \\
\hline 1890 & C1ty of Newark & PA, PN \\
\hline 1928 & $\begin{array}{l}\text { Morris Canal and } \\
\text { Banking Co }\end{array}$ & $\mathrm{PA}$ ef \\
\hline 1925 & $\begin{array}{l}\text { Morris Canal and } \\
\text { Banking Co }\end{array}$ & - \\
\hline 1927 & Arthur D Crane Co & - \\
\hline 1917 & C1ty of Newark & $\mathrm{PA}, \mathrm{PN}$ \\
\hline 1922 & Hackensack Water 60 & PA \\
\hline 1948 & Jersey C1ty & - \\
\hline 1870 & $\begin{array}{l}\text { Miliville Manufac- } \\
\text { turing Co }\end{array}$ & - \\
\hline 1928 & $\begin{array}{l}\text { North Jersey Dist } \\
\text { Water Supply } \\
\text { Commission }\end{array}$ & - \\
\hline 1963 & Corps of Engineers & PA \\
\hline 1938 & $\begin{array}{l}\text { Bureau of } \\
\text { Reclamation }\end{array}$ & PA \\
\hline 1927 & $\begin{array}{l}\text { Bluewater-Tol tec } \\
\text { Irrigation Dist }\end{array}$ & PA \\
\hline 1938 & $\begin{array}{l}\text { Bureav of } \\
\text { Reclamation }\end{array}$ & $\mathrm{PA}$ \\
\hline 1939 & Corps of Engineers & PA \\
\hline 19 & $\begin{array}{l}\text { Costilla Water } \\
\text { Users Assoc }\end{array}$ & PA \\
\hline
\end{tabular}


Table 1 --Reservo1rs in the United States completed or under constmuction as of January 1, 1963, having a usable capac1ty of 5,000 acre-feet or more--Cont1nued [For meaning of symbols under "Use" and "Storage records published," see text]

\begin{tabular}{|c|c|c|c|c|c|c|c|c|c|c|c|c|}
\hline \multirow[b]{2}{*}{$\begin{array}{c}\text { Name of reservolr } \\
\text { and } \\
\text { stream }\end{array}$} & \multicolumn{2}{|c|}{ Location } & \multirow{2}{*}{$\begin{array}{l}\text { Drainage } \\
\text { area } \\
\text { (square } \\
\text { m1les) }\end{array}$} & \multirow[b]{2}{*}{$\begin{array}{c}\text { Surface } \\
\text { area } \\
\text { (acres) }\end{array}$} & \multicolumn{3}{|c|}{ Storage in acre-feet } & \multirow[b]{2}{*}{$\begin{array}{c}\text { Storage } \\
\text { rat10 }\end{array}$} & \multirow[b]{2}{*}{ Use } & \multirow[b]{2}{*}{$\begin{array}{c}\text { Date } \\
\text { com- } \\
\text { pleted }\end{array}$} & \multirow[b]{2}{*}{$\begin{array}{l}\text { Owner } \\
\text { or } \\
\text { operator }\end{array}$} & \multirow{2}{*}{$\begin{array}{l}\text { Storage } \\
\text { records } \\
\text { pub- } \\
\text { lished }\end{array}$} \\
\hline & $\begin{array}{c}\text { Basin } \\
\text { 1ndex and } \\
\text { station no }\end{array}$ & $\begin{array}{l}\text { Latitude } \\
\text { and } \\
\text { long1tude }\end{array}$ & & & Dead & Total & Usable & & & & & \\
\hline NEW MEXIC0--Cont Inued & & & & & & & & & & & & \\
\hline $\begin{array}{l}\text { Eagle Nest } \\
\text { Cimarron Creek }\end{array}$ & $07-2055^{7 C B} 00$ & $\begin{array}{rr}36 & 32 \\
105 & 14\end{array}$ & 167 & 2,426 & 0 & 79,120 & 79,120 & 5 & IR & 1918 & $\begin{array}{l}\text { Springer Land and } \\
\text { Cattle Co }\end{array}$ & PA \\
\hline $\begin{array}{l}\text { El Vado } \\
\text { Río Chama }\end{array}$ & $08-2850 \quad 00$ & $\begin{array}{rr}36 & 36 \\
106 & 44\end{array}$ & 873 & 3,230 & 0 & 194,500 & 194,500 & 7 & FIR & 1935 & $\begin{array}{l}\text { Middle Rio Grande } \\
\text { Conservancy Dist }\end{array}$ & PA \\
\hline $\begin{array}{l}\text { Elephant Butte } \\
\text { Rio Grande }\end{array}$ & O8-3605 80 & $\begin{array}{rr}33 & 09 \\
107 & 11\end{array}$ & 29,445 & 36,584 & 0 & $2,195,000$ & $2,195,000$ & 29 & FIPR & 1916 & $\begin{array}{l}\text { Bureau of } \\
\text { Reclamation }\end{array}$ & PA \\
\hline $\begin{array}{l}\text { Jemez Canyon } \\
\text { Jemez River }\end{array}$ & $08-328500$ & $\begin{array}{rr}35 & 24 \\
106 & 33\end{array}$ & 1,034 & 2,895 & o & 114,000 & 114,000 & 30 & $\mathrm{DF}$ & 1953 & Corps of Engineers & PA \\
\hline $\begin{array}{l}\text { La Cueva-Red Lakes } \\
\text { Mora River } \underline{\text { c/ }}\end{array}$ & $07-2153 \begin{array}{r}7 C D \\
00\end{array}$ & $\begin{array}{rr}35 & 57 \\
105 & 13\end{array}$ & - & 477 & 0 & 5,070 & 5,070 & - & I & $1938 a$ & William Salman & - \\
\hline $\begin{array}{l}\text { Lake Avalon } \\
\text { Pecos River }\end{array}$ & $08-403800$ & $\begin{array}{rr}32 & 29 \\
104 & 15\end{array}$ & 18,070 & 947 & o & 5,080 & 5,080 & $<05$ & IR & 1906 & $\begin{array}{l}\text { Bureau of } \\
\text { Reclamation }\end{array}$ & $\mathrm{PA}$ \\
\hline $\begin{array}{l}\text { Lake Isabel } \\
\text { Sapello River c/ }\end{array}$ & $07-2202$ TCD & $\begin{array}{rr}35 & 45 \\
105 & 09\end{array}$ & - & 827 & 0 & 6,500 & 6,500 & - & I & 1922 & E F Shellaberger & - \\
\hline $\begin{array}{l}\text { Lake McMilian } \\
\text { Pecos River }\end{array}$ & $08-400500$ & \begin{tabular}{rr|}
32 & 36 \\
104 & 21
\end{tabular} & 16,990 & 5,695 & 0 & 39,430 & 39,430 & 5 & FI & 1893 & $\begin{array}{l}\text { Bureau of } \\
\text { Reclamation }\end{array}$ & PA \\
\hline $\begin{array}{l}\text { Maxwell No } 13 \\
\text { Vermejo River and Curtis Creek c/ }\end{array}$ & $07-2037^{7 \mathrm{CA}}$ & $\begin{array}{rr}36 & 35 \\
104 & 35\end{array}$ & - & 332 & 0 & 5,000 & 5,000 & - & I & 1953 & $\begin{array}{l}\text { Verme jo Conservancy } \\
\text { D1strict }\end{array}$ & - \\
\hline $\begin{array}{l}\text { Morgan Lake } \\
\text { San Juan River c/ }\end{array}$ & $09-3676^{9 \mathrm{GC}}$ & $\begin{array}{rr}36 & 42 \\
108 & 30\end{array}$ & - & 1,269 & 3,018 & 39,570 & 36,550 & - & RW & 1962 & $\begin{array}{l}\text { Utah Construction } \\
\text { Co }\end{array}$ & - \\
\hline $\begin{array}{l}\text { Navajo } \\
\text { San Juan River }\end{array}$ & $\begin{array}{r}9 \mathrm{GA} \\
09-355100\end{array}$ & \begin{tabular}{rr|}
36 & 49 \\
107 & 37
\end{tabular} & 3,230 & 15,630 & - & $1,709,000$ & $1,036,000$ & 18 & DFIR & 1962 & $\begin{array}{l}\text { Bureau of } \\
\text { Reclamation }\end{array}$ & PA \\
\hline $\begin{array}{l}\text { Ramah } \\
\text { Zun1 Arroyo (Pescado Creek) }\end{array}$ & $09-3868$ 9J 00 & $\begin{array}{rr}35 & 09 \\
108 & 29\end{array}$ & 57 & - & - & 13,500 & 13,500 & - & CI & 1944 & $\begin{array}{l}\text { Ramah Land and } \\
\text { Irrigation Co }\end{array}$ & - \\
\hline $\begin{array}{l}\text { Red Lake } \\
\text { Black Wash }\end{array}$ & 09-3958 9 ०० & $\begin{array}{rr}36 & 55 \\
109 & 02\end{array}$ & a250 & 908 & 217 & 9,740 & 9,520 & - & FIRW & 1954 & $\begin{array}{l}\text { Nava jo Tribe, Win- } \\
\text { dow Rock, Ariz }\end{array}$ & - \\
\hline $\begin{array}{l}\text { Storrie } \\
\text { Gallinas River } c /\end{array}$ & $-{ }^{8 F B}$ & $\begin{array}{rr}35 & 40 \\
105 & 14\end{array}$ & - & 1,084 & 2,806 & 24,533 & 21,727 & - & IR & 1939 & $\begin{array}{l}\text { Storrie Project } \\
\text { Water Users Assoc }\end{array}$ & - \\
\hline $\begin{array}{l}\text { Stubblefiela } \\
\text { Vermejo River c/ }\end{array}$ & $08-2036$ 7CA & $\begin{array}{rr}36 & 34 \\
104 & 40\end{array}$ & - & 1,250 & 26 & 16,720 & 16,695 & - & I & 1953 & $\begin{array}{l}\text { Verme jo Conservancy } \\
\text { District }\end{array}$ & - \\
\hline $\begin{array}{l}\text { Two Rivers } \\
\text { R10 Hondo and Rocky Arroyo }\end{array}$ & $\left|\begin{array}{r}8 \mathrm{FI} \\
08-3906 \text { O0 }\end{array}\right|$ & $\begin{array}{rr}33 & 18 \\
104 & 44\end{array} \mid$ & 1,027 & 4,806 & 0 & 167,900 & 167,900 & 6 & $\mathrm{~F}$ & 1963 & Corps of Engineers & PA \\
\hline
\end{tabular}

政




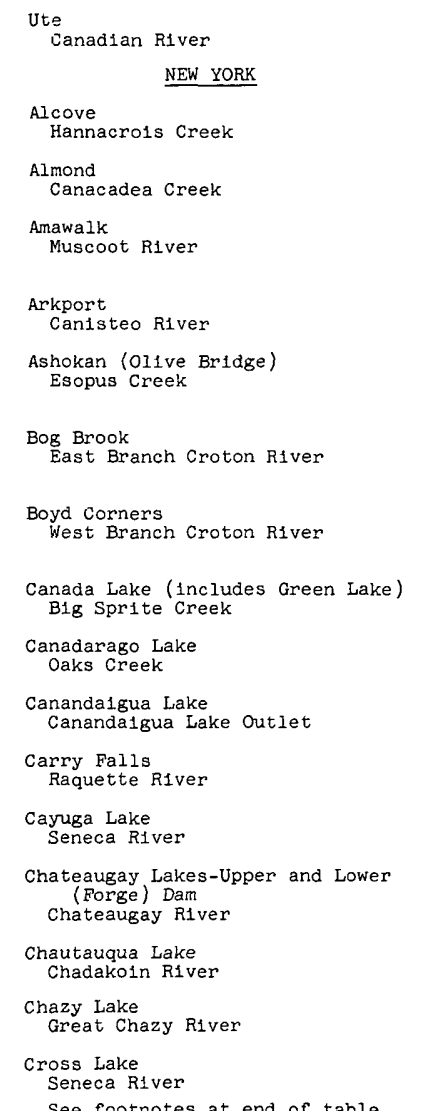

\begin{tabular}{|c|c|c|c|}
\hline $\begin{array}{r}7 \mathrm{CJ} \\
07-2268 \text { O0 }\end{array}$ & $\begin{array}{rr}35 & 21 \\
103 & 26\end{array}$ & 11,140 & 4,150 \\
\hline $01-3599^{1 \mathrm{JE}}$ & $\begin{array}{ll}42 & 28 \\
73 & 56\end{array}$ & $\begin{array}{ll}32 & 6\end{array}$ & - \\
\hline $01-5230^{1 N H}$ & $\begin{array}{ll}42 & 21 \\
77 & 42\end{array}$ & $\begin{array}{ll}56 & 0\end{array}$ & 490 \\
\hline $01-3749 \stackrel{1 K F}{40}$ & $\begin{array}{ll}41 & 17 \\
73 & 46\end{array}$ & 191 & 606 \\
\hline $01-5210^{1 \mathrm{NH}}$ & $\begin{array}{ll}42 & 24 \\
77 & 43\end{array}$ & $\begin{array}{lll}30 & 5\end{array}$ & 190 \\
\hline $\begin{array}{r}1 K B \\
01-363400\end{array}$ & $\begin{array}{ll}41 & 56 \\
74 & 13\end{array}$ & 257 & 8,180 \\
\hline $01-3745^{1 \mathrm{KF}}$ & $\begin{array}{ll}41 & 24 \\
73 & 36\end{array}$ & $\begin{array}{ll}3 & 7\end{array}$ & 399 \\
\hline $\begin{array}{r}1 \mathrm{KF} \\
01-3745^{80}\end{array}$ & $\begin{array}{ll}41 & 27 \\
73 & 44\end{array}$ & 225 & 297 \\
\hline $\begin{array}{r}1 \mathrm{JG} \\
01-3473 \text { OO }\end{array}$ & $\begin{array}{ll}43 & 09 \\
74 & 36\end{array}$ & $\begin{array}{ll}41 & 9\end{array}$ & al, 100 \\
\hline $\begin{array}{l}\text { INA } \\
01-496450\end{array}$ & $\begin{array}{ll}42 & 48 \\
75 & 01\end{array}$ & a65 & 1,958 \\
\hline $\begin{array}{r}4 \mathrm{NC} \\
04-2345 \stackrel{00}{ }\end{array}$ & $\begin{array}{ll}42 & 52 \\
77 & 16\end{array}$ & 189 & 10,600 \\
\hline $\begin{array}{r}40 F \\
04-2667 \text { DO }\end{array}$ & $\begin{array}{ll}44 & 26 \\
74 & 44\end{array}$ & 872 & 1,370 \\
\hline $\begin{array}{r}4 \mathrm{NB} \\
04-2340 \quad 40\end{array}$ & $\begin{array}{ll}42 & 57 \\
76 & 44\end{array}$ & 1,587 & 42,500 \\
\hline $\begin{array}{r}4 \mathrm{OH} \\
04-27040 \mathrm{O}\end{array}$ & $\begin{array}{ll}44 & 50 \\
74 & 03\end{array}$ & - & 1,810 \\
\hline $\begin{array}{r}3 \mathrm{AC} \\
03-013500\end{array}$ & $\begin{array}{ll}42 & 14 \\
79 & 30\end{array}$ & 189 & 13,376 \\
\hline $\begin{array}{r}4 P A \\
04-270900\end{array}$ & $\begin{array}{ll}44 & 45 \\
73 & 49\end{array}$ & 22 & 1,802 \\
\hline $\begin{array}{r}\text { 4ND } \\
04-237400\end{array}$ & $\begin{array}{ll}43 & 09 \\
76 & 20\end{array}$ & 3,130 & 2,176 \\
\hline
\end{tabular}

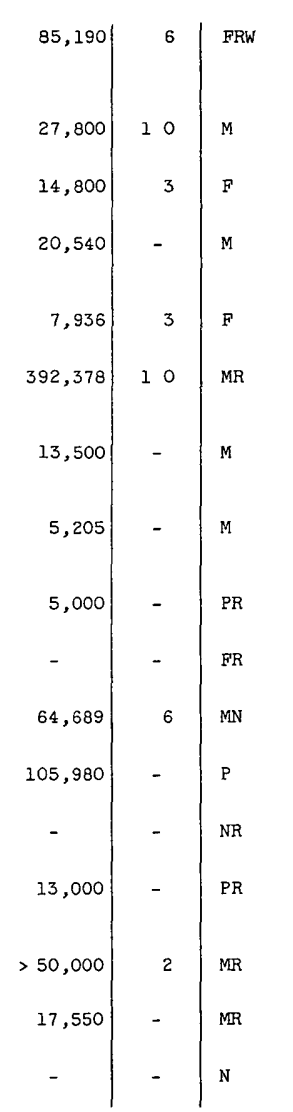


Table 1 --Reservo1rs in the United States completed or under construction as of January 1, 1963, hav1ng a usable capac1ty of 5,000 acre-feet or more--Continued

\begin{tabular}{|c|c|c|c|c|c|c|c|c|c|c|c|c|}
\hline \multirow[b]{2}{*}{$\begin{array}{l}\text { Name of reservo1r } \\
\text { and } \\
\text { stream }\end{array}$} & \multicolumn{2}{|c|}{ Location } & \multirow{2}{*}{$\begin{array}{c}\text { Drainage } \\
\text { area } \\
\text { (square } \\
\text { m1les) }\end{array}$} & \multirow[b]{2}{*}{$\begin{array}{c}\text { Surface } \\
\text { area } \\
\text { (acres) }\end{array}$} & \multicolumn{3}{|c|}{ Storage in acre-feet } & \multirow[b]{2}{*}{$\mid \begin{array}{c}\text { Storage } \\
\text { rat10 }\end{array}$} & \multirow[b]{2}{*}{ Use } & \multirow[b]{2}{*}{$\left|\begin{array}{c}\text { Date } \\
\text { com- } \\
\text { pleted }\end{array}\right|$} & \multirow[b]{2}{*}{$\begin{array}{l}\text { Owner } \\
\text { or } \\
\text { operator }\end{array}$} & \multirow{2}{*}{$\begin{array}{l}\text { Storage } \\
\text { records } \\
\text { pub- } \\
\text { l1shed }\end{array}$} \\
\hline & \begin{tabular}{|c|} 
Bas1n \\
index and \\
station no.
\end{tabular} & $\begin{array}{l}\text { Lat1 tude } \\
\text { and } \\
\text { long1 tude }\end{array}$ & & & Dead & Total & Usable & & & & & \\
\hline NEW YORK--ContInued & & & & & & & & & & & & \\
\hline $\begin{array}{l}\text { Cross River } \\
\text { Cross River }\end{array}$ & 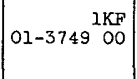 & $\begin{array}{ll}41 & 16 \\
73 & 40\end{array} \mid$ & 298 & 769 & - & - & 31,630 & - & M & 1908 & $\begin{array}{c}\text { New York C1ty Dept } \\
\text { of Water Supply, } \\
\text { Gas, and Elec }\end{array}$ & $P G$ \\
\hline $\begin{array}{l}\text { Croton Falls } \\
\text { Mlddle Branch Croton River }\end{array}$ & $\begin{array}{r}1 \mathrm{KF} \\
01-374700\end{array}$ & $\begin{array}{ll}41 & 22 \\
73 & 40\end{array} \mid$ & 1686 & 1,062 & - & - & 43,550 & - & M & 1911 & $\begin{array}{l}\text { New York C1ty Dept } \\
\text { of Water Supply, } \\
\text { Gas, and Elec }\end{array}$ & $P G$ \\
\hline $\begin{array}{l}\text { Croton Lake (New Croton Dam) } \\
\text { Croton R1ver }\end{array}$ & $01-3749_{95}^{1 K F}$ & $\begin{array}{ll}41 & 14 \\
73 & 51\end{array} \mid$ & 375 & 2,259 & 18,790 & 100,480 & 81,690 & 02 & M & 1905 & $\begin{array}{c}\text { New York C1ty Dept } \\
\text { of Water Suppiy, } \\
\text { Gas, and Elec }\end{array}$ & PG \\
\hline $\begin{array}{l}\text { Delta } \\
\text { Mohawk River }\end{array}$ & $01-3359$ IJF & $\begin{array}{ll}43 & 16 \\
75 & 26\end{array}$ & 145 & 2,750 & - & - & 64,300 & 2 & FN & 1910 & $\begin{array}{l}\text { New York State Dept } \\
\text { of Publlic Works }\end{array}$ & - \\
\hline $\begin{array}{l}\text { East Branch (Sodom) } \\
\text { East Branch Croton River }\end{array}$ & $01-3744 \quad 1 K F$ & $\begin{array}{ll}11 & 23 \\
73 & 36\end{array}$ & 803 & 557 & - & - & 16,090 & - & M & 1891 & $\begin{array}{c}\text { New York C1ty Dept } \\
\text { of Water Supply, } \\
\text { Gas, and Elec }\end{array}$ & $P G$ \\
\hline $\begin{array}{l}\text { East Sidney } \\
\text { Ouleout Creek }\end{array}$ & $\begin{array}{r}1 \mathrm{NB} \\
01-499500\end{array}$ & $\begin{array}{ll}42 & 20 \\
75 & 14\end{array}$ & 102 & 2,100 & 56 & 33,600 & 33,550 & 3 & FR & 1950 & Corps of Engineers & PA \\
\hline $\begin{array}{l}\text { Forked Lake } \\
\text { Raquette R1ver }\end{array}$ & $\begin{array}{r}40 F \\
04-2654 \quad 60\end{array}$ & $\begin{array}{ll}43 & 54 \\
74 & 32\end{array}$ & - & 1,250 & - & - & a5, 000 & - & - & - & $\begin{array}{l}\text { Wh1 tney Industries, } \\
\text { Inc }\end{array}$ & - \\
\hline $\begin{array}{l}\text { Gates } \\
\text { Gates Creek }\end{array}$ & $\mid \begin{array}{r}3 \mathrm{AA} \\
03-0107 \quad 60\end{array}$ & $\begin{array}{ll}42 & 19 \\
78 & 26\end{array}$ & - & - & - & 7,060 & - & - & $\mathrm{F}$ & 1961 & $\begin{array}{l}\text { Ischua Creek County } \\
\text { Watershed Protec- } \\
\text { tion District }\end{array}$ & - \\
\hline $\begin{array}{l}\text { Hemlock Lake } \\
\text { Hem } \perp \text { Lake Outlet }\end{array}$ & $04-228920$ & $\begin{array}{ll}42 & 47 \\
77 & 37\end{array}$ & 462 & 1,856 & - & 22,300 & - & - & M & - & - & - \\
\hline $\begin{array}{l}\text { Hinckley } \\
\text { West Canada Creek }\end{array}$ & $01-3439$ OJ & $\begin{array}{ll}43 & 18 \\
75 & 06\end{array}$ & 373 & 3,270 & - & 79,000 & 76,200 & 1 & FMNP & 1916 & $\begin{array}{l}\text { New York State Dept } \\
\text { of Publ1n Works }\end{array}$ & $\mathrm{PA}$ \\
\hline $\begin{array}{l}\text { Honeoye Lake } \\
\text { Honeoye Creek }\end{array}$ & $\begin{array}{r}4 M F \\
04-228850\end{array}$ & $\begin{array}{ll}42 & 47 \\
78 & 00\end{array}$ & a70 & 1,670 & - & 19,300 & - & - & M & 1890 & - & - \\
\hline $\begin{array}{l}\text { Indian Lake } \\
\text { Indian River }\end{array}$ & $\begin{array}{r}1 J A \\
01-314500\end{array}$ & $\begin{array}{ll}43 & 45 \\
74 & 17\end{array}$ & 131 & 5,035 & - & 114,000 & 103,000 & 5 & FMNP & 1898 & Ind1an R1ver Co & PA \\
\hline $\begin{array}{l}\text { Kensico } \\
\text { Bronx River }\end{array}$ & $\begin{array}{r}1 \mathrm{HE} \\
01-301900\end{array}$ & $\begin{array}{ll}41 & 05 \\
73 & 46\end{array}$ & 128 & 2,218 & 6,490 & 96,040 & 89,550 & - & M & 1915 & $\begin{array}{l}\text { New York C1ty Dept } \\
\text { of Water Supply, } \\
\text { Gas, and Elec }\end{array}$ & $P G$ \\
\hline $\begin{array}{l}\text { Keuka Lake } \\
\text { Keuka Lake Outlet }\end{array}$ & 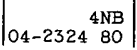 & $\begin{array}{ll}42 & 40 \\
77 & 03\end{array}$ & 179 & 11,155 & - & - & b11, 000 & 07 & FMNPR & 1875 & - & - \\
\hline
\end{tabular}




\begin{tabular}{|c|c|c|c|c|c|c|c|c|c|c|c|c|}
\hline $\begin{array}{l}\text { Lake DeForest } \\
\text { Hackensack River }\end{array}$ & $\left|\begin{array}{r}1 K H \\
01-376700\end{array}\right|$ & $\begin{array}{ll}41 & 06 \\
73 & 57\end{array}$ & 266 & 1,016 & 921 & 17,303 & 16,382 & 4 & M & 1956 & $\begin{array}{l}\text { Spring Valley water } \\
\text { Works and Supply } \\
\text { Co }\end{array}$ & $\mathrm{PA}$ \\
\hline $\begin{array}{l}\text { Lake George } \\
\text { Lake George Outlet }\end{array}$ & O4-2789 $\mathrm{OP}$ & $\begin{array}{ll}43 & 48 \\
73 & 27\end{array} \mid$ & 262 & 28,442 & - & - & 285,000 & 4 & FMNPR & 1844 & - & - \\
\hline $\begin{array}{l}\text { Little Tupper Lake (Includes Round } \\
\text { Lake) } \\
\text { Raquette RIver }\end{array}$ & $\begin{array}{r}40 F \\
04-2659 O 0\end{array}$ & $\begin{array}{ll}44 & 02 \\
74 & 37\end{array}$ & $\mathrm{a} 60$ & 3,206 & - & - & a19,000 & 2 & FPR & 1900 & $\begin{array}{l}\text { Whitney Industries, } \\
\text { Inc }\end{array}$ & - \\
\hline $\begin{array}{l}\text { Lower Saranac Lake (State Locks) } \\
\text { Saranac R1ver }\end{array}$ & $\begin{array}{r}4 \mathrm{~PB} \\
04-2720 \quad 20\end{array}$ & $\begin{array}{ll}44 & 17 \\
74 & 09\end{array}$ & 146 & 2,210 & - & - & a5, 900 & - & $\mathrm{R}$ & - & - & PA \\
\hline $\begin{array}{l}\text { Lows Lake (Bog River Flow) } \\
\text { Bog River }\end{array}$ & $04-265950$ & $\begin{array}{ll}44 & 07 \\
74 & 40\end{array}$ & - & - & - & - & a20,000 & - & - & - & A Augustus Low & - \\
\hline $\begin{array}{l}\text { Middile Branch } \\
\text { Middle Branch Croton R1ver }\end{array}$ & $\mid \begin{array}{r}1 K F \\
01-3746 \quad 60\end{array}$ & $\begin{array}{ll}41 & 23 \\
73 & 39\end{array} \mid$ & 213 & 428 & - & - & 12,290 & - & M & 1878 & $\begin{array}{l}\text { New York City Dept } \\
\text { of Water Supply, } \\
\text { Gas, and Elec }\end{array}$ & $P G$ \\
\hline $\begin{array}{l}\text { Mount Morris } \\
\text { Genesee River }\end{array}$ & $04-2240 \quad 00$ & $\begin{array}{ll}42 & 44 \\
77 & 55\end{array}$ & 1,077 & 3,680 & 609 & 435,000 & 337,000 & 3 & $\mathrm{~F}$ & 1951 & Corps of Engineers & PA \\
\hline $\begin{array}{l}\text { Muscoot } \\
\text { Croton River }\end{array}$ & $\begin{array}{r}1 \mathrm{KF} \\
01-374980\end{array}$ & $\begin{array}{ll}41 & 17 \\
73 & 45\end{array}$ & 3157 & 1,166 & - & - & 15,080 & - & M & 1905 & $\begin{array}{l}\text { New York City Dept } \\
\text { of Water Supply, } \\
\text { Gas, and Elec }\end{array}$ & $\mathrm{PG}$ \\
\hline $\begin{array}{l}\text { Neversink } \\
\text { Neversink River }\end{array}$ & | 1 1LE & $\begin{array}{ll}41 & 49 \\
74 & 38\end{array}$ & 918 & 1,500 & 5,165 & 214,004 & 108,839 & 6 & MPR & 1953 & $\begin{array}{l}\text { New York C1ty Dept } \\
\text { of Water Suppiy, } \\
\text { Gas, and Elec }\end{array}$ & $\mathrm{PG}$ \\
\hline $\begin{array}{l}\text { North Lake } \\
\quad \text { North Branch Black R1ver }\end{array}$ & $\begin{array}{r}4 \mathrm{NH} \\
04-250800\end{array}$ & $\begin{array}{ll}43 & 32 \\
74 & 56\end{array}$ & 31 & 396 & - & 6,900 & - & - & $\mathrm{N}$ & $1857 \mathrm{a}$ & - & - \\
\hline $\begin{array}{l}\text { Old Forge } \\
\text { Middle Branch Moose River }\end{array}$ & $\begin{array}{r}4 \mathrm{NH} \\
04-253490\end{array}$ & $\begin{array}{ll}43 & 43 \\
74 & 56\end{array}$ & 52 & 3,150 & - & $\mathrm{d} 21,000$ & - & - & FMPRW & 1880 & State of New York & - \\
\hline $\begin{array}{l}\text { One1da Lake (Caughdenoy Dam) } \\
\text { One1da River }\end{array}$ & $\begin{array}{r}4 \mathrm{NE} \\
04-2466 \mathrm{OO}\end{array}$ & $\begin{array}{ll}43 & 16 \\
76 & 12\end{array}$ & 1,377 & 51,072 & - & 270,000 & - & - & NR & 1910 & $\begin{array}{l}\text { New York State Dept } \\
\text { of Public Works }\end{array}$ & PA \\
\hline $\begin{array}{l}\text { Onondaga } \\
\text { Onondaga Creek }\end{array}$ & $\begin{array}{r}4 \mathrm{ND} \\
04-2380\end{array}$ & $\begin{array}{ll}42 & 56 \\
76 & 10\end{array}$ & 681 & - & 0 & 18,200 & 18,200 & 3 & $\mathrm{~F}$ & 1949 & Corps of Engineers & PA \\
\hline $\begin{array}{l}\text { Otsego Lake } \\
\text { Susquehanna RIver }\end{array}$ & $01-4963$ INA & $\begin{array}{ll}42 & 42 \\
74 & 55\end{array}$ & a75 & 3,987 & - & $a 46,000$ & - & - & FMR & 1850 & $\begin{array}{c}\text { Cooperstown Mun1c1- } \\
\text { pal Water Works }\end{array}$ & - \\
\hline $\begin{array}{l}\text { Owasco Lake } \\
\text { Owasco Lake Outlet }\end{array}$ & $\begin{array}{r}4 \mathrm{ND} \\
04-235400\end{array}$ & $\begin{array}{ll}42 & 55 \\
76 & 33\end{array}$ & 205 & 6,592 & - & 34,000 & - & - & FMNPR & - & State of New York & - \\
\hline $\begin{array}{l}\text { Peck Lake } \\
\text { Peck Creek }\end{array}$ & $01-3482$ 1JC & $\begin{array}{ll}43 & 06 \\
74 & 26\end{array}$ & 118 & al, 400 & - & - & 23,000 & - & PR & - & $\begin{array}{l}\text { N1agara Mohawk } \\
\text { Power Co }\end{array}$ & - \\
\hline $\begin{array}{l}\text { Pepacton } \\
\text { East Branch Delaware River }\end{array}$ & $01-4169$ OLB & $\begin{array}{ll}42 & 04 \\
74 & 58\end{array}$ & 371 & 6,400 & 5,831 & 459,711 & 453,880 & 8 & MPR & 1954 & $\begin{array}{l}\text { New York City Dept } \\
\text { of Water Supply, } \\
\text { Gas and Elec }\end{array}$ & $P A$ \\
\hline
\end{tabular}




\begin{tabular}{|c|c|c|c|c|c|c|c|c|c|c|c|c|}
\hline \multirow[b]{2}{*}{$\begin{array}{l}\text { Name of reservo1r } \\
\text { and } \\
\text { stream }\end{array}$} & \multicolumn{2}{|c|}{ Location } & \multirow{2}{*}{$\begin{array}{l}\text { Drainage } \\
\text { area } \\
\text { (square } \\
\text { m1les) }\end{array}$} & \multirow[b]{2}{*}{$\begin{array}{c}\text { Surface } \\
\text { area } \\
\text { (acres) }\end{array}$} & \multicolumn{3}{|c|}{ Storage in acre-feet } & \multirow[b]{2}{*}{$\left|\begin{array}{c}\text { Storage } \\
-4 t 10\end{array}\right|$} & \multirow[b]{2}{*}{ Use } & \multirow[b]{2}{*}{$\begin{array}{c}\text { Date } \\
\text { com- } \\
\text { pleted }\end{array}$} & \multirow[b]{2}{*}{$\begin{array}{l}\text { Owner } \\
\text { or } \\
\text { operator }\end{array}$} & \multirow{2}{*}{$\begin{array}{l}\text { Storage } \\
\text { records } \\
\text { pub- } \\
\text { 11shed }\end{array}$} \\
\hline & \begin{tabular}{|c|} 
Basin \\
index and \\
station no.
\end{tabular} & $\begin{array}{l}\text { Lat1tude } \\
\text { and } \\
\text { long1 tude }\end{array}$ & & & Dead & Total & Usable & & & & & \\
\hline NEW YORK--Continued & & & & & & & & & & & & \\
\hline $\begin{array}{l}\text { Ra1nbow Falls } \\
\text { Racquette R1ver }\end{array}$ & 04-2669 $\begin{array}{r}40 \mathrm{~F} \\
\end{array}$ & $\begin{array}{ll}44 & 31 \\
74 & 49\end{array}$ & - & 715 & - & 12,600 & - & - & $\mathrm{P}$ & 1956 & $\begin{array}{l}\text { Niagara Mohawk } \\
\text { Power Co }\end{array}$ & - \\
\hline $\begin{array}{l}\text { Rondout } \\
\text { Rondout Creek }\end{array}$ & $01-366400$ & $\begin{array}{ll}41 & 48 \\
74 & 26\end{array} \mid$ & $94 \quad 4$ & 2,100 & 7,323 & 162,167 & 154,844 & 09 & MR & 1955 & $\begin{array}{l}\text { New York C1ty Dept } \\
\text { of Water Supply, } \\
\text { Gas, and Elec }\end{array}$ & $\mathrm{PA}$ \\
\hline $\begin{array}{l}\text { Rushford Lake } \\
\text { Caneadea Creek }\end{array}$ & $04-221999_{90}^{4 M D}$ & $\begin{array}{ll}42 & 23 \\
78 & 11\end{array}$ & 607 & 577 & $<1,000$ & 25,400 & $>24,400$ & 4 & $P$ & 1928 & $\begin{array}{l}\text { Rochester Gas and } \\
\text { Electr1c Corp }\end{array}$ & PA \\
\hline $\begin{array}{l}\text { Sacandaga } \\
\text { Sacandaga River }\end{array}$ & $01-323500$ & $\begin{array}{ll}43 & 19 \\
73 & 56\end{array}$ & 1,044 & 26,700 & 106,000 & 868,000 & 762,000 & 5 & FNPR & 1930 & $\begin{array}{l}\text { Hudson River Regu- } \\
\text { lating District }\end{array}$ & PA \\
\hline $\begin{array}{l}\text { Salmon River } \\
\text { Salmon RIver }\end{array}$ & 04-2497 $4 \mathrm{NF}$ & $\begin{array}{ll}43 & 33 \\
75 & 55\end{array}$ & 191 & 3,290 & 0 & $\mathrm{~d} 61,100$ & $d 61,100$ & 2 & $\mathrm{P}$ & 1913 & $\begin{array}{l}\text { Central New York } \\
\text { Power Corp }\end{array}$ & - \\
\hline $\begin{array}{l}\text { Sand Lake } \\
\text { Woodhull Creek }\end{array}$ & $04-25095 \mathrm{NH}$ & $\begin{array}{ll}43 & 34 \\
75 & 00\end{array}$ & 13 & 333 & - & 5,500 & - & - & FNP & 1857 & - & - \\
\hline $\begin{array}{l}\text { Saratoga Lake } \\
\text { F1sh Creek }\end{array}$ & $01-330900$ & $\begin{array}{ll}43 & 06 \\
73 & 38\end{array}$ & 244 & 24,300 & - & - & 8,800 & - & $\mathrm{PR}$ & 1911 & $\begin{array}{l}\text { N1agara Mohawk } \\
\text { Power Co }\end{array}$ & - \\
\hline $\begin{array}{l}\text { Saunders } \\
\text { Saunders Creek }\end{array}$ & $\mid \begin{array}{r}3 \mathrm{AA} \\
03-0107 \quad 50\end{array}$ & $\begin{array}{ll}42 & 21 \\
78 & 26\end{array} \mid$ & - & - & - & 5,140 & - & - & $F$ & 1962 & $\begin{array}{l}\text { Ischua Creek County } \\
\text { Small Watershed } \\
\text { Protection D1st }\end{array}$ & - \\
\hline $\begin{array}{l}\text { Schoharie (Gilboa Dam) } \\
\text { Schoharle Creek }\end{array}$ & $\mid \begin{array}{r}1 \mathrm{HH} \\
01-3501 \\
00\end{array}$ & $\begin{array}{ll}42 & 23 \\
74 & 27\end{array}$ & 314 & 1,145 & 6,037 & 66,139 & 60,102 & 1 & MR & 1926 & $\begin{array}{l}\text { New York C1ty Dept } \\
\text { of Water Supply, } \\
\text { Gas, and Elec }\end{array}$ & PA \\
\hline $\begin{array}{l}\text { Schroon Lake (Starbuckville Dam) } \\
\text { Schroon River }\end{array}$ & $\begin{array}{r}1 \mathrm{JB} \\
01-3168 \text { 50 }\end{array}$ & $\begin{array}{ll}43 & 41 \\
73 & 47\end{array}$ & - & 24,300 & - & - & a13,000 & - & - & - & - & - \\
\hline $\begin{array}{l}\text { Seneca Lake } \\
\text { Seneca River }\end{array}$ & $\begin{array}{r}4 \mathrm{NB} \\
04-232650\end{array}$ & $\begin{array}{ll}42 & 54 \\
76 & 52\end{array}$ & 714 & 42,688 & - & - & al 72,000 & 2 & FMNPR & $1912 a$ & $\begin{array}{l}\text { New York State Dept } \\
\text { of Public Works }\end{array}$ & PA \\
\hline $\begin{array}{l}\text { Sixth Lake } \\
\text { M1ddle Branch Moose River }\end{array}$ & $04-2533^{4 N H}$ & $\begin{array}{ll}43 & 44 \\
74 & 46\end{array}$ & 17 & 736 & - & 6,800 & - & - & FMRW & 1880 & $\begin{array}{l}\text { Black River Regu- } \\
\text { lating District }\end{array}$ & - \\
\hline $\begin{array}{l}\text { Skaneateles Lake } \\
\text { Skaneateles Lake Outlet }\end{array}$ & $\begin{array}{r}4 N D \\
04-2360 \\
00\end{array}$ & $\begin{array}{ll}42 & 57 \\
76 & 26\end{array}$ & 738 & 8,832 & - & 50,000 & - & - & M & - & - & - \\
\hline $\begin{array}{l}\text { South Lake } \\
\text { South Branch Black River }\end{array}$ & (04-2508 $4 \mathrm{NH}$ & $\begin{array}{ll}43 & 31 \\
74 & 53\end{array}$ & 6 & 500 & - & 9,200 & - & - & FNP & 1857 & - & - \\
\hline $\begin{array}{l}\text { Stark } \\
\text { Racquette River }\end{array}$ & $\left|\begin{array}{r}4 O F \\
04-266800\end{array}\right|$ & $\begin{array}{ll}44 & 27 \\
77 & 46\end{array}$ & - & 861 & - & 12,000 & - & - & $\mathrm{P}$ & 1957 & $\begin{array}{l}\text { N1agara Mohawk } \\
\text { Power Co }\end{array}$ & - \\
\hline
\end{tabular}




\begin{tabular}{|c|c|c|c|c|c|c|c|c|c|c|c|c|}
\hline $\begin{array}{l}\text { Stillwater } \\
\text { Beaver River }\end{array}$ & $\mid \begin{array}{r}4 \mathrm{NH} \\
04-256500\end{array}$ & $\begin{array}{ll}43 & 54 \\
75 & 03\end{array}$ & 172 & 6,700 & 2,070 & 108,200 & 106,100 & 4 & FP & 1885 & \begin{tabular}{|} 
Black River Regu- \\
lating District
\end{tabular} & PA \\
\hline $\begin{array}{l}\text { Swing1ng Br1dge } \\
\text { Mongaup R1ver }\end{array}$ & $01-4330$ 1LE & $\begin{array}{ll}41 & 34 \\
74 & 47\end{array}$ & 118 & 1,000 & 4,883 & 37,860 & 32,980 & 1 & $\mathrm{P}$ & 1930 & $\begin{array}{c}\text { Orange and Rockland } \\
\text { Ut1lities, Inc }\end{array}$ & PA \\
\hline $\begin{array}{l}\text { Taylor Pond } \\
\text { Black Brook }\end{array}$ & $\begin{array}{r}4 \mathrm{~PB} \\
04-2744 \quad 00\end{array}$ & $\begin{array}{ll}44 & 29 \\
73 & 52\end{array}$ & 11 & 602 & - & al1,500 & - & - & FPR & 1905 & $\begin{array}{l}\text { New York State Elec. } \\
\text { and Gas Corp }\end{array}$ & - \\
\hline $\begin{array}{l}\text { Titicus } \\
\text { Titicus River }\end{array}$ & $\begin{array}{r}1 \mathrm{KF} \\
01-3748 \quad 20\end{array}$ & $\begin{array}{ll}41 & 19 \\
73 & 39\end{array}$ & 233 & 669 & - & - & 21,990 & - & M & 1893 & $\begin{array}{l}\text { New York C1ty Dept } \\
\text { of Water Supply, } \\
\text { Gas, and Elec }\end{array}$ & $P G$ \\
\hline $\begin{array}{l}\text { Tomhannock } \\
\text { Tomhannock Creek }\end{array}$ & $01-335200$ & $\begin{array}{ll}42 & 50 \\
73 & 32\end{array}$ & 67 & 1,470 & - & 37,000 & 36,700 & 4 & M & 1904 & City of Troy & - \\
\hline $\begin{array}{l}\text { Toronto } \\
\text { Black Lake Creek }\end{array}$ & $01-4331$ 1LE & $\begin{array}{ll}41 & 37 \\
74 & 50\end{array}$ & 232 & 800 & 615 & 25,826 & 25,211 & 8 & $P$ & 1926 & $\begin{array}{l}\text { Orange and Rockland } \\
\text { Utilities, Inc }\end{array}$ & $\mathrm{PA}$ \\
\hline $\begin{array}{l}\text { Tupper Lake-includes Raquette Pond } \\
\text { and S1mon Pond (Setting Pole } \\
\text { Rapids Dam) } \\
\text { Raquette River }\end{array}$ & $\begin{array}{r}40 F \\
04-2664 \quad 00\end{array}$ & $\begin{array}{ll}44 & 14 \\
74 & 32\end{array}$ & - & 5,340 & - & - & $>30,000$ & - & $\mathrm{PR}$ & 1935 & $\begin{array}{l}\text { Town of Altamont, } \\
\text { Paul Smith's Elec } \\
\text { L1ght, Power, and } \\
\text { Railroad Co }\end{array}$ & - \\
\hline $\begin{array}{l}\text { West Branch (Carmel) } \\
\text { West Branch Croton River }\end{array}$ & $01-374620$ & $\begin{array}{ll}41 & 25 \\
73 & 42\end{array}$ & 429 & 1,083 & - & - & 30,900 & - & M & 1895 & $\begin{array}{l}\text { New York C1ty Dept } \\
\text { of Water Supply, } \\
\text { Cas, and Elec }\end{array}$ & PG \\
\hline $\begin{array}{l}\text { Whitney Point } \\
\text { Otselic R1ver }\end{array}$ & 01-5110 00 & $\begin{array}{ll}42 & 20 \\
75 & 58\end{array}$ & 255 & 3,350 & 28 & 86,470 & 86,440 & 2 & $\mathrm{FR}$ & 1942 & Corps of Engineers & $\mathrm{PA}$ \\
\hline $\begin{array}{l}\text { Woodhull Lake } \\
\text { Woodhull Creek }\end{array}$ & $\begin{array}{r}4 \mathrm{NH} \\
04-250940\end{array}$ & $\begin{array}{ll}43 & 36 \\
74 & 59\end{array}$ & 6 & 1,120 & - & 9,200 & - & - & FNP & 1857 & - & - \\
\hline NORTH CAROLINA & & & & & & & & & & & & \\
\hline $\begin{array}{l}\text { Apalachia } \\
\text { Hiwassee River }\end{array}$ & \begin{tabular}{rr|}
$3 \mathrm{UJ}$ \\
$03-5555 \quad 00$
\end{tabular} & $\begin{array}{ll}35 & 10 \\
84 & 18\end{array}$ & 1,018 & 1,123 & 8,520 & 58,600 & 50,080 & $<05$ & FNP & 1943 & $\begin{array}{l}\text { Tennessee Valley } \\
\text { Author1ty }\end{array}$ & $P A, P E$ \\
\hline $\begin{array}{l}\text { Badin Lake (Narrows) } \\
\text { Yadkin River }\end{array}$ & $02-1228 \quad 44$ & $\begin{array}{ll}35 & 25 \\
80 & 06\end{array}$ & $a 4,180$ & 5,973 & 98,608 & 241,000 & 142,400 & $<05$ & $\mathrm{P}$ & 1917 & $\begin{array}{c}\text { adkin, Inc (Caro- } \\
\text { lina Aluminum Co })\end{array}$ & PA \\
\hline $\begin{array}{l}\text { Blewett Falls Lake } \\
\text { Pee Dee River }\end{array}$ & $02-128800$ & $\begin{array}{ll}35 & 59 \\
79 & 53\end{array}$ & a6, 830 & 2,500 & 54,530 & 97,000 & 42,500 & $<05$ & $\mathrm{P}$ & 1911 & $\begin{array}{l}\text { Carolina Power and } \\
\text { Light Co }\end{array}$ & $\mathrm{PA}$ \\
\hline $\begin{array}{l}\text { Burnett Lake } \\
\text { North Fork Swannanoa R1ver }\end{array}$ & $03-448959$ & $\begin{array}{ll}35 & 40 \\
82 & 21\end{array}$ & 219 & 372 & 0 & 17,500 & 17,500 & 5 & M & 1954 & C1ty of Asheville & - \\
\hline $\begin{array}{l}\text { Chatuge } \\
\text { H1wassee R1ver }\end{array}$ & $\begin{array}{r}3 \mathrm{UJ} \\
03-546500\end{array}$ & $\begin{array}{ll}35 & 01 \\
83 & 47\end{array}$ & 189 & 7,150 & 18,500 & 247,800 & 229,300 & 7 & FNPR & 1942 & $\begin{array}{l}\text { Tennessee Valley } \\
\text { Authority }\end{array}$ & $\mathrm{PA}, \mathrm{PE}$ \\
\hline $\begin{array}{l}\text { Fontana } \\
\text { Little Tennessee River }\end{array}$ & $\begin{array}{r}3 \mathrm{TP} \\
03-514500\end{array}$ & $\begin{array}{ll}35 & 27 \\
83 & 48\end{array}$ & 1,571 & 10,670 & 287,000 & $1,444,300$ & $1,157,300$ & 4 & FNPR & 1944 & $\begin{array}{l}\text { Tennessee Valley } \\
\text { Author1ty }\end{array}$ & $\mathrm{PA}, \mathrm{PE}$ \\
\hline $\begin{array}{l}\text { High Point Municipal Lake } \\
\text { Deep River }\end{array}$ & $\left|02-0990 \begin{array}{r}2 \mathrm{EC} \\
96\end{array}\right|$ & $\begin{array}{ll}35 & 60 \\
79 & 57\end{array} \mid$ & 614 & - & - & 5,064 & - & - & M & 1926 & City of High Point & - \\
\hline
\end{tabular}


Table 1 --Reservo1rs in the United States completed or under construction as of January 1, 1963, having a usable capac1ty of 5,000 acre-feet or more--Continued

\begin{tabular}{|c|c|c|c|c|c|c|c|c|c|c|c|c|}
\hline \multirow[b]{2}{*}{$\begin{array}{l}\text { Name of reservo1r } \\
\text { and } \\
\text { stream }\end{array}$} & \multicolumn{2}{|c|}{ Location } & \multirow{2}{*}{$\begin{array}{c}\text { Dra1nage } \\
\text { area } \\
\text { (square } \\
\text { m1les) }\end{array}$} & \multirow[b]{2}{*}{$\begin{array}{c}\text { Surface } \\
\text { area } \\
\text { (acres) }\end{array}$} & \multicolumn{3}{|c|}{ Storage in acre-feet } & \multirow[b]{2}{*}{$\mid \begin{array}{c}\text { Storage } \\
\text { rat10 }\end{array}$} & \multirow[b]{2}{*}{ Use } & \multirow[b]{2}{*}{$\begin{array}{c}\text { Date } \\
\text { com- } \\
\text { pleted }\end{array}$} & \multirow[b]{2}{*}{$\begin{array}{l}\text { Owner } \\
\text { or } \\
\text { operator }\end{array}$} & \multirow{2}{*}{$\begin{array}{l}\text { Storage } \\
\text { records } \\
\text { pub- } \\
\text { lished }\end{array}$} \\
\hline & \begin{tabular}{|c|} 
Basin \\
1ndex and \\
station no
\end{tabular} & $\begin{array}{l}\text { Lat1tude } \\
\text { and } \\
\text { long1tude }\end{array}$ & & & Dead & Total & Usable & & & & & \\
\hline \multicolumn{13}{|l|}{ NORTH CAROLINA- Cont Inued } \\
\hline $\begin{array}{l}\text { High Rock Lake } \\
\text { Yadk1n River }\end{array}$ & $02-122400$ & $\begin{array}{ll}35 & 36 \\
80 & 14\end{array}$ & a4,, 000 & 15,180 & 19,743 & 254,591 & 234,848 & 007 & $\mathrm{P}$ & 1927 & $\begin{array}{c}\text { Yadkin, Ine (Caro- } \\
\text { lina Aluminum Co) }\end{array}$ & PA \\
\hline $\begin{array}{l}\text { Hiwassee } \\
\text { Hiwassee River }\end{array}$ & $03-554500$ & $\begin{array}{ll}35 & 09 \\
84 & 11\end{array}$ & 968 & 6,280 & 73,300 & 438,000 & 364,700 & 3 & FNPR & 1940 & $\begin{array}{l}\text { Tennessee Valley } \\
\text { Authority }\end{array}$ & $\mathrm{PA}, \mathrm{PE}$ \\
\hline $\begin{array}{l}\text { Lake Brandt } \\
\text { Reedy Fork and Horsepen Creek }\end{array}$ & $\begin{array}{r}2 E A \\
02-0941 \quad 17\end{array}$ & $\begin{array}{ll}36 & 10 \\
79 & 50\end{array}$ & 270 & - & - & 6,750 & - & - & $M$ & 1960 & C1ty of Greensboro & - \\
\hline $\begin{array}{l}\text { Lake Gaston } \\
\text { Roanoke Fiver }\end{array}$ & $02-0799^{2 \mathrm{CF}} 64$ & $\begin{array}{ll}36 & 30 \\
77 & 49\end{array}$ & 8,339 & 22,000 & 53,000 & 514,000 & 457,000 & 1 & FP & 1963 & $\begin{array}{l}\text { Virginia Electric } \\
\text { and Power Co }\end{array}$ & $\mathrm{PA}$ \\
\hline $\begin{array}{l}\text { Lake H1ckory (Oxford) } \\
\text { Catawba River }\end{array}$ & $02-1419^{2 \mathrm{~GB}} 61$ & $\begin{array}{ll}35 & 49 \\
81 & 11\end{array}$ & al, 310 & 4,110 & 49,922 & 127,479 & 77,557 & $<05$ & $P$ & 1928 & Duke Power Co & $\mathrm{PA}$ \\
\hline $\begin{array}{l}\text { Lake James (Bridgewater Dam) } \\
\text { Catawba and Linville Rivers and } \\
\text { Paddy Creek }\end{array}$ & $02-1381^{2 G A}$ & $\begin{array}{ll}35 & 46 \\
81 & 52\end{array}$ & 380 & 6,500 & 47,649 & 288,843 & 241,194 & 5 & $P$ & 1919 & Duke Power Co & $\mathrm{PA}$ \\
\hline $\begin{array}{l}\text { Lake Michie } \\
\text { Flat River }\end{array}$ & $02-0864 \quad 90$ & $\begin{array}{ll}36 & 09 \\
78 & 50\end{array}$ & a170 & 550 & 1,010 & 14,187 & 13,177 & 1 & MP & 1926 & City of Durham & - \\
\hline $\begin{array}{l}\text { Lahe Norman (Cowans Ford) } \\
\text { Catawba R1ver }\end{array}$ & $02-1426 \quad 47$ & $\begin{array}{ll}35 & 26 \\
80 & 58\end{array}$ & a1,790 & 32,510 & 474,651 & $1,092,429$ & 617,778 & 3 & $P$ & 1962 & Duke Power Co & $\mathrm{PA}$ \\
\hline $\begin{array}{l}\text { Lake Tiliery (Norwood) } \\
\text { Pee Dee River }\end{array}$ & $02-1237^{2 \mathrm{FF}}$ & $\begin{array}{ll}35 & 12 \\
80 & 04\end{array}$ & 24,600 & 5,000 & 30,934 & 167,000 & 136,100 & $<05$ & $\mathrm{P}$ & 1928 & $\begin{array}{l}\text { Carolina Power and } \\
\text { Light co }\end{array}$ & $\mathrm{PA}$ \\
\hline $\begin{array}{l}\text { Lake Walters } \\
\text { P1geon River }\end{array}$ & $03-4602^{3 \mathrm{TH}}$ & $\begin{array}{ll}35 & 42 \\
83 & 03\end{array}$ & 455 & 340 & 4,780 & 25,280 & 20,500 & $<05$ & $P$ & 1929 & $\begin{array}{l}\text { Carolina Power and } \\
\text { Light Co }\end{array}$ & - \\
\hline $\begin{array}{l}\text { Lake Wheeler } \\
\text { Swift Creek }\end{array}$ & $02-0875^{2 D B}$ & $\begin{array}{ll}35 & 42 \\
78 & 42\end{array}$ & 38 & 540 & - & 6,138 & - & - & $\mathrm{MR}$ & 1956 & City of Raleigh & - \\
\hline $\begin{array}{l}\text { Lexington-Thomasville } \\
\text { Abbotts Creek }\end{array}$ & $02-1214 \quad 61$ & $\begin{array}{ll}35 & 52 \\
80 & 12\end{array}$ & 703 & - & 61 & 6,522 & 6,461 & 1 & M & 1957 & $\begin{array}{l}\text { Cities of Thomas- } \\
\text { ville and } \\
\text { Lexington }\end{array}$ & - \\
\hline $\begin{array}{l}\text { Lookout Shoals Lake } \\
\text { Catawba R1ver }\end{array}$ & $02-1424 \quad 2 G B$ & $\begin{array}{ll}35 & 46 \\
81 & 06\end{array}$ & al, 450 & 1,270 & 22,197 & 31,121 & 8,914 & $<05$ & $\mathrm{P}$ & 1915 & Duke Power co & $\mathrm{PA}$ \\
\hline $\begin{array}{l}\text { Mt Island Lake } \\
\text { Catawba River }\end{array}$ & $\begin{array}{r}2 \mathrm{~GB} \\
02-142676\end{array}$ & $\begin{array}{ll}35 & 20 \\
80 & 59\end{array}$ & al, 860 & 3,235 & 31,313 & 57,300 & 26,000 & $<05$ & $P$ & 1923 & Duke Power Co & PA \\
\hline $\begin{array}{l}\text { Nantahala Lake } \\
\text { Nantahala River }\end{array}$ & $\left|\begin{array}{r}3 \mathrm{TN} \\
03-504500\end{array}\right|$ & $\begin{array}{ll}35 & 12 \\
83 & 39\end{array}$ & 910 & 1,605 & b12,700 & 138,700 & 126,000 & $<05$ & $P$ & 1942 & $\mid \begin{array}{c}\text { Nantahala Power and } \\
\text { L1ght Co }\end{array}$ & $\mathrm{PA}, \mathrm{PE}$ \\
\hline
\end{tabular}




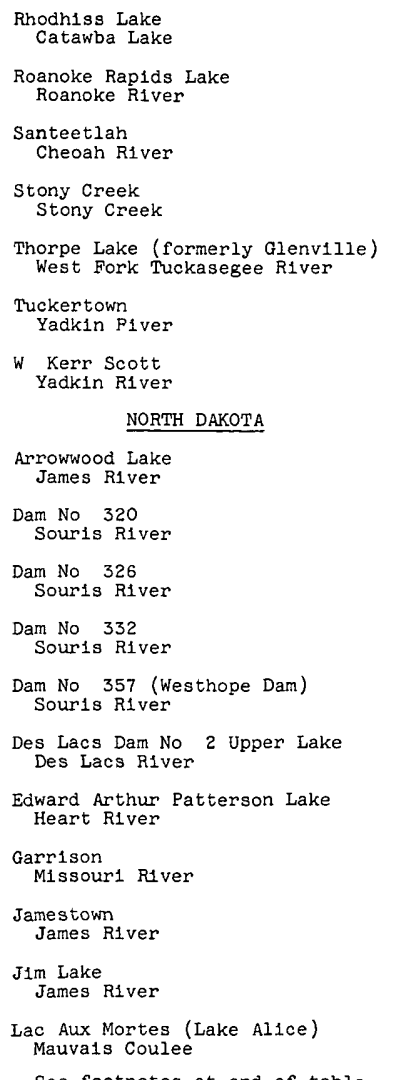

\begin{tabular}{|c|c|c|c|c|}
\hline $\begin{array}{r}2 \mathrm{gA} \\
02-141490\end{array}$ & $\begin{array}{ll}35 & 47 \\
81 & 27\end{array}$ & al,, 090 & 3,515 & 33,783 \\
\hline $02-080100$ & $\begin{array}{ll}36 & 29 \\
77 & 40\end{array}$ & 8,395 & - & 52,700 \\
\hline $\begin{array}{r}3 \mathrm{TP} \\
03-516500\end{array}$ & $\begin{array}{ll}35 & 23 \\
83 & 54\end{array}$ & 176 & 2,863 & 25,000 \\
\hline $02-0960^{2 E B}$ & $\begin{array}{ll}36 & 10 \\
79 & 26\end{array}$ & 44 & 755 & 0 \\
\hline $\begin{array}{r}370 \\
03-507500\end{array}$ & $\begin{array}{ll}35 & 12 \\
83 & 09\end{array}$ & 367 & 1,462 & 3,720 \\
\hline $02-1226 \quad \begin{array}{r}2 F D \\
99\end{array}$ & $\begin{array}{ll}35 & 29 \\
80 & 10\end{array}$ & $a 4,120$ & 2,529 & 35,782 \\
\hline $02-1113^{2 F C} 91$ & $\begin{array}{ll}36 & 08 \\
81 & 13\end{array}$ & a350 & 3,980 & 8,000 \\
\hline $\begin{array}{r}6 \mathrm{LO} \\
06-4684 \text { 00 }\end{array}$ & $\begin{array}{ll}47 & 15 \\
98 & 51\end{array}$ & 120 & 1,600 & - \\
\hline $\begin{array}{r}5 R E \\
05-123460\end{array}$ & $\begin{array}{r}4835 \\
10039\end{array}$ & - & $a 3,000$ & 0 \\
\hline $\begin{array}{r}5 R E \\
05-123470\end{array}$ & $\begin{array}{rr}48 & 38 \\
100 & 43\end{array}$ & - & $a 2,500$ & 0 \\
\hline $\begin{array}{r}\text { 5RG } \\
05-123850\end{array}$ & $\begin{array}{rr}48 & 40 \\
100 & 47\end{array}$ & - & - & 0 \\
\hline $05-123990$ & $\begin{array}{rr}48 & 59 \\
100 & 58\end{array}$ & 17,600 & a2, 500 & 0 \\
\hline $05-1161 \quad 30$ & $\begin{array}{rr}48 & 42 \\
102 & 06\end{array}$ & - & 4,000 & - \\
\hline $06-3435$ OJM & $\begin{array}{rr}46 & 52 \\
102 & 49\end{array}$ & 400 & 867 & 1,100 \\
\hline $\begin{array}{r}6 \mathrm{JJ} \\
06-3380 \text { 00 }\end{array}$ & \begin{tabular}{rr|}
47 & 30 \\
101 & 25
\end{tabular} & 181,000 & 390,000 & $4,900,000$ \\
\hline $\begin{array}{r}6 L 0 \\
06-4690 \quad 00\end{array}$ & $\begin{array}{ll}46 & 56 \\
98 & 42\end{array}$ & 1,245 & 13,200 & 10,000 \\
\hline $\begin{array}{r}\text { 6LO } \\
06-468450\end{array}$ & $\begin{array}{ll}47 & 10 \\
98 & 47\end{array}$ & - & 1,689 & - \\
\hline 05-056250 & $\begin{array}{ll}48 & 19 \\
99 & 07\end{array}$ & 704 & 3,420 & - \\
\hline
\end{tabular}

$\begin{array}{r}73,200 \\ 80,700 \\ 158,200 \\ 9,820 \\ 70,800 \\ 42,526 \\ 153,000 \\ 8,450 \\ 9,890 \\ 5,958 \\ 5,371 \\ 19,790 \\ 55,000 \\ 7,000 \\ 230,000 \\ 7,550 \\ -800,000 \\ \hline\end{array}$

\begin{tabular}{r|c|l}
39,400 & $<05$ & $\mathrm{P}$ \\
28,000 & $<05$ & $\mathrm{P}$ \\
133,200 & 3 & $\mathrm{FR}$ \\
9,820 & 3 & $\mathrm{M}$ \\
67,080 & 8 & $\mathrm{FP}$ \\
6,744 & $<05$ & $\mathrm{P}$ \\
145,000 & 3 & $\mathrm{~F}$ \\
- & - & $\mathrm{C}$ \\
9,890 & - & $\mathrm{C}$ \\
5,958 & - & $\mathrm{C}$ \\
5,371 & - & $\mathrm{C}$ \\
19,790 & - & $\mathrm{C}$ \\
- & - & $\mathrm{C}$ \\
5,900 & 2 & $\mathrm{FIM}$ \\
$13,900,000$ & - & $\mathrm{FINPR}$ \\
220,000 & - & $\mathrm{CFM}$ \\
- & - & $\mathrm{C}$ \\
- & $\mathrm{C}$ \\
\hline & & \\
\hline & & \\
\hline
\end{tabular}

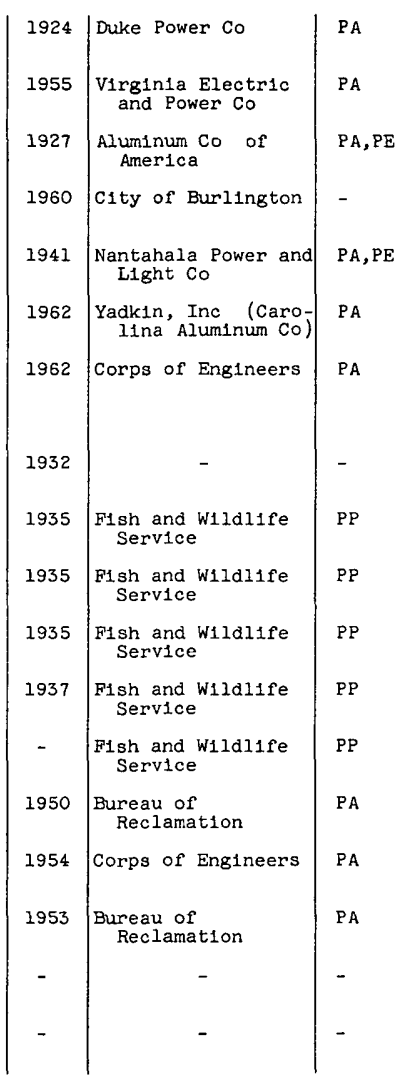




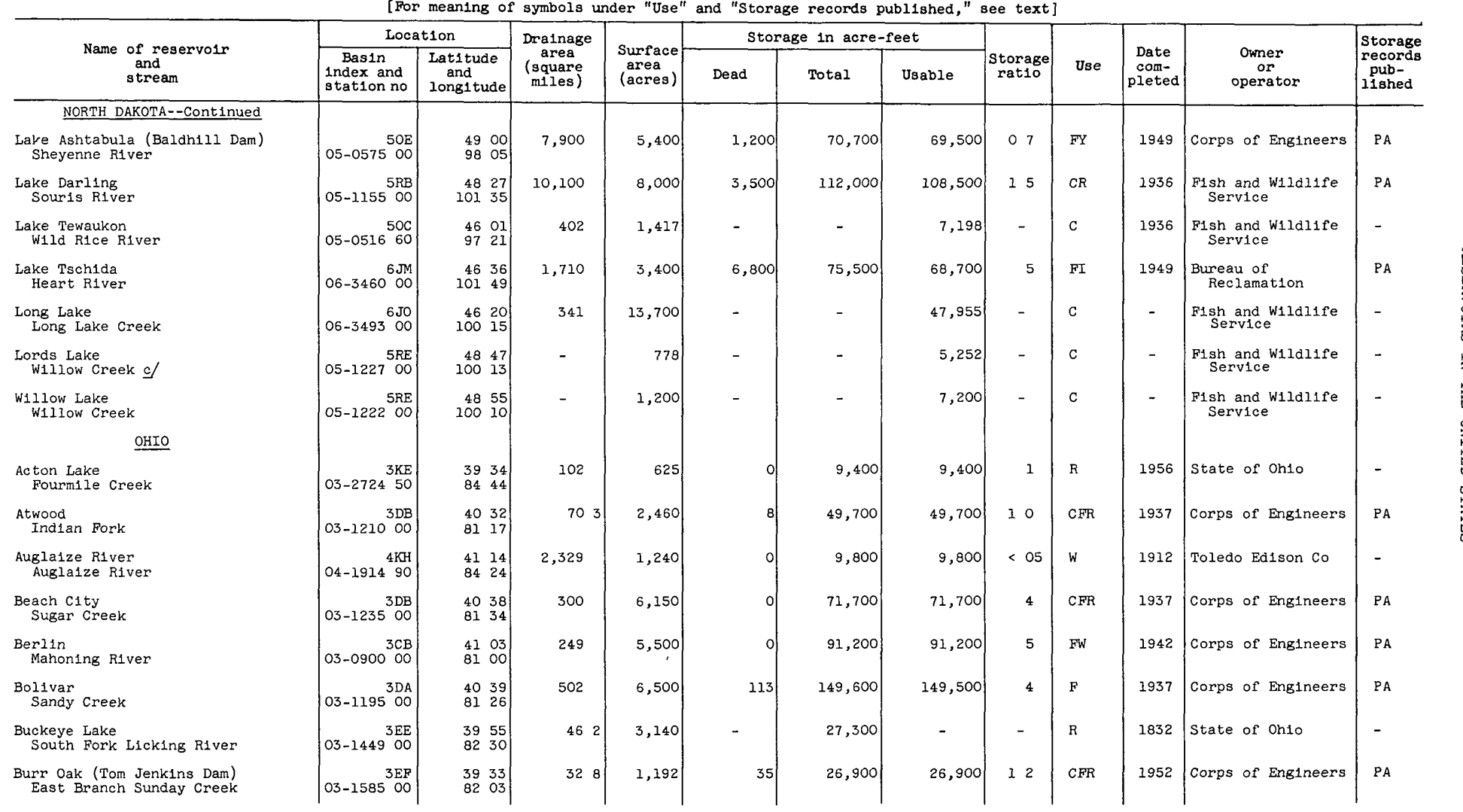




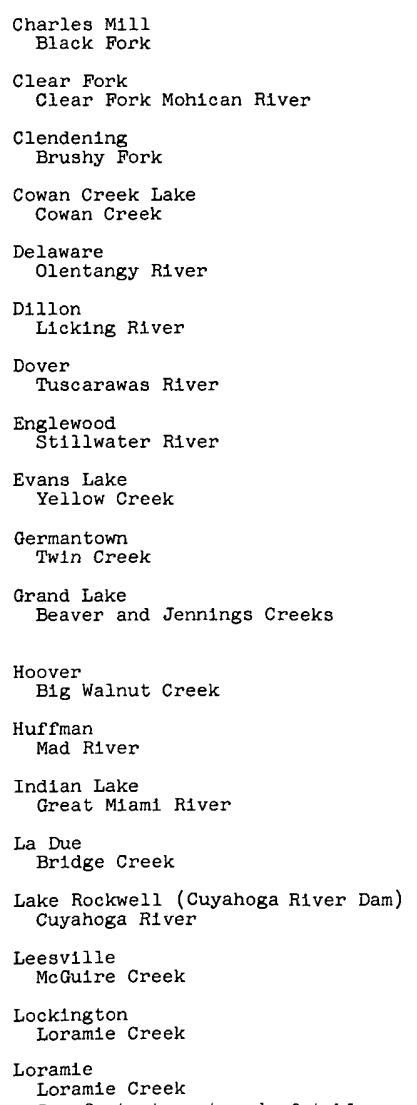

\begin{tabular}{|c|c|c|c|c|c|}
\hline $\begin{array}{r}3 \mathrm{DD} \\
03-129500\end{array}$ & $\begin{array}{ll}40 & 44 \\
82 & 22\end{array}$ & 216 & 6,050 & 310 & 88,000 \\
\hline $03-1319$ ODD & $\begin{array}{ll}40 & 42 \\
82 & 36\end{array}$ & 350 & 997 & o & 10,700 \\
\hline $\begin{array}{r}3 D C \\
03-126500\end{array}$ & $\begin{array}{ll}40 & 16 \\
81 & 17\end{array}$ & 695 & 2,620 & 27 & 54,000 \\
\hline $03-2438$ OJE & $\begin{array}{ll}39 & 23 \\
83 & 55\end{array} \mid$ & 51 & 648 & o & 12,000 \\
\hline $03-2250 \quad 00$ & $\begin{array}{ll}40 & 21 \\
83 & 04\end{array}$ & 381 & 8,700 & 0 & 132,000 \\
\hline $\begin{array}{r}3 D H \\
03-1473 \quad 00\end{array}$ & $\begin{array}{ll}40 & 00 \\
82 & 05\end{array}$ & 748 & 10,285 & 30 & 274,000 \\
\hline $\begin{array}{r}3 \mathrm{DB} \\
03-1220 \\
00\end{array}$ & $\begin{array}{ll}40 & 34 \\
81 & 25\end{array}$ & 1,397 & 10,100 & 0 & 203,000 \\
\hline $03-2659 \begin{array}{r}3 \mathrm{~KB} \\
90\end{array}$ & $\begin{array}{ll}39 & 52 \\
84 & 17\end{array}$ & 646 & 7,900 & 0 & 312,000 \\
\hline $\begin{array}{r}3 \mathrm{CB} \\
03-0990 \quad 00\end{array}$ & $\begin{array}{ll}40 & 59 \\
80 & 37\end{array}$ & 106 & 580 & 0 & $\mathrm{~d} 10,500$ \\
\hline $\begin{array}{r}3 \mathrm{KD} \\
03-271990\end{array}$ & $\begin{array}{ll}39 & 38 \\
84 & 24\end{array}$ & 275 & 3,600 & o & 106,000 \\
\hline $\begin{array}{r}4 \mathrm{KE} \\
04-1809 \quad 30\end{array}$ & $\begin{array}{ll}40 & 32 \\
84 & 34\end{array}$ & 114 & 13,440 & - & 92,000 \\
\hline $\begin{array}{r}3 \mathrm{HD} \\
03-2284 \quad 00\end{array}$ & $\begin{array}{ll}40 & 06 \\
82 & 53\end{array}$ & 190 & 2,825 & 224 & 60,342 \\
\hline $\begin{array}{r}3 \mathrm{KC} \\
03-2699 \quad 90\end{array}$ & $\begin{array}{ll}39 & 48 \\
84 & 06\end{array}$ & 635 & - & of & 167,000 \\
\hline $\begin{array}{r}3 \mathrm{KA} \\
03-2605 \quad 00\end{array}$ & $\begin{array}{ll}40 & 28 \\
83 & 53\end{array}$ & 110 & 6,134 & - & 45,900 \\
\hline $04-2019 \stackrel{4 \mathrm{LF}}{\mathrm{OO}}$ & $\begin{array}{ll}41 & 24 \\
81 & 12\end{array}$ & 278 & 1,500 & o) & 18,110 \\
\hline $\begin{array}{r}4 \mathrm{LF} \\
04-202400\end{array}$ & $\begin{array}{ll}41 & 11 \\
81 & 20\end{array}$ & 207 & 769 & 1,380 & 7,060 \\
\hline $\begin{array}{r}3 \mathrm{DB} \\
03-1200 \quad 00\end{array}$ & $\begin{array}{ll}40 & 28 \\
81 & 12\end{array}$ & 479 & 1,470 & 329 & 37,400 \\
\hline $\begin{array}{r}3 \mathrm{KKA} \\
03-261990\end{array}$ & $\begin{array}{ll}40 & 13 \\
84 & 15\end{array}$ & 261 & - & 0 & 70,000 \\
\hline$\left|\begin{array}{r}3 K A \\
03-2618 \text { O० }\end{array}\right|$ & $\begin{array}{ll}40 & 22 \\
84 & 22\end{array} \mid$ & 70 & 1,700 & - & $a 13,000$ \\
\hline
\end{tabular}

\begin{tabular}{|c|c|c|}
\hline 87,700 & 6 & $\mathrm{CFR}$ \\
\hline 10,700 & 4 & M \\
\hline 54,000 & 10 & $\mathrm{CFR}$ \\
\hline 12,000 & 3 & $R$ \\
\hline 132,000 & 5 & CFMR \\
\hline 274,000 & 5 & $\mathrm{~F}$ \\
\hline 203,000 & 2 & $C F$ \\
\hline 312,000 & 7 & F \\
\hline d10,500 & 15 & W \\
\hline 106,000 & 6 & $\mathrm{~F}$ \\
\hline - & - & $\mathrm{RW}$ \\
\hline 60,128 & 4 & MR \\
\hline 167,000 & 4 & $F$ \\
\hline - & - & $\mathrm{R}$ \\
\hline 18,110 & 7 & M \\
\hline$d 6,900$ & $<05$ & M \\
\hline 37,100 & 10 & $\mathrm{CF}$ \\
\hline 70,000 & 5 & $\mathrm{~F}$ \\
\hline- & - & $R$ \\
\hline
\end{tabular}

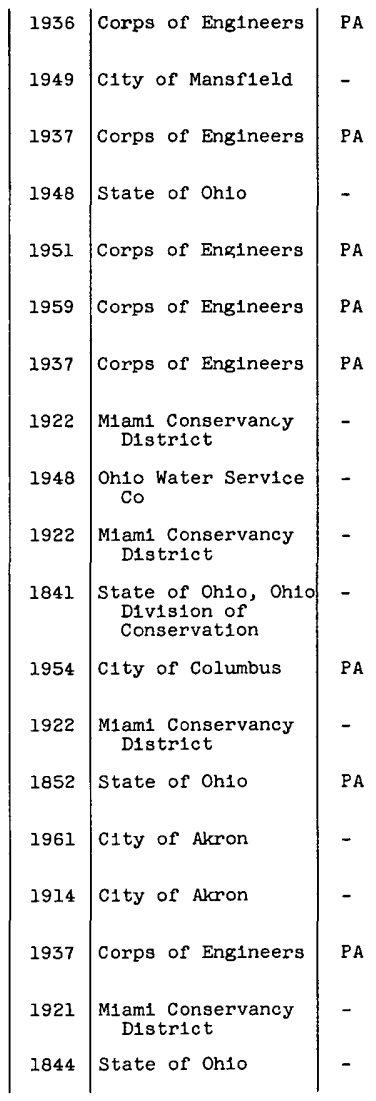




\begin{tabular}{|c|c|c|c|c|c|c|c|c|c|c|c|c|}
\hline \multirow[b]{2}{*}{$\begin{array}{l}\text { Name of reservolr } \\
\text { and } \\
\text { stream }\end{array}$} & \multicolumn{2}{|c|}{ Location } & \multirow{2}{*}{$\begin{array}{c}\text { Drainage } \\
\text { area } \\
\text { (square } \\
\text { m1les) }\end{array}$} & \multirow[b]{2}{*}{$\begin{array}{c}\text { Surface } \\
\text { area } \\
\text { (acres) }\end{array}$} & \multicolumn{3}{|c|}{ Storage in acre-feet } & \multirow[b]{2}{*}{$\mid \begin{array}{c}\text { Storage } \\
\text { rat10 }\end{array}$} & \multirow[b]{2}{*}{ Use } & \multirow[b]{2}{*}{$\begin{array}{c}\text { Date } \\
\text { com- } \\
\text { pleted }\end{array}$} & \multirow[b]{2}{*}{$\begin{array}{l}\text { Owner } \\
\text { or } \\
\text { operator }\end{array}$} & \multirow{2}{*}{$\begin{array}{c}\text { Storage } \\
\text { records } \\
\text { pub- } \\
\text { l1shed }\end{array}$} \\
\hline & \begin{tabular}{|c|} 
Basin \\
1ndex and \\
station no \\
\end{tabular} & $\begin{array}{c}\text { Lat1 tude } \\
\text { and } \\
\text { long1 tude }\end{array}$ & & & Dead & Total & Usable & & & & & \\
\hline oHIO--Cont1 nued & & & & & & & & & & & & \\
\hline $\begin{array}{l}\text { Meander Creek } \\
\text { Meander Creek }\end{array}$ & $\begin{array}{r}3 \mathrm{CB} \\
03-0970 \quad 00\end{array}$ & $\begin{array}{ll}41 & 09 \\
80 & 47\end{array}$ & 849 & 2,010 & 0 & 32,410 & 32,410 & 05 & м & 1929 & $\begin{array}{l}\text { Mahoning Valley } \\
\text { Santtary Distr1ct }\end{array}$ & PA \\
\hline $\begin{array}{l}\text { Milton } \\
\text { Mahoning River }\end{array}$ & $\begin{array}{r}3 \mathrm{CB} \\
03-0910 \quad 00\end{array}$ & $\begin{array}{ll}41 & 08 \\
80 & 59\end{array}$ & 276 & 1,660 & 0 & 29,150 & 29,150 & 2 & $\mathrm{RW}$ & 1916 & C1ty of Youngstown & $\mathrm{PA}$ \\
\hline $\begin{array}{l}\text { Mogadore } \\
\text { L1ttle Cuyahoga R1ver }\end{array}$ & $04-203990$ & $\begin{array}{ll}41 & 04 \\
81 & 22\end{array}$ & 143 & 900 & 368 & 6,900 & 6,530 & 7 & RW & 1939 & C1ty of Akron & - \\
\hline $\begin{array}{l}\text { Mohawk } \\
\text { Walhonding River }\end{array}$ & $03-1380 \quad 00$ & $\begin{array}{ll}40 & 21 \\
82 & 05\end{array}$ & 1,501 & 7,950 & 59 & 285,000 & 285,000 & 3 & F & 1937 & Corps of Eng1neers & PA \\
\hline $\begin{array}{l}\text { Mohicanville } \\
\text { Lake Fork }\end{array}$ & $03-1345$ ODD & $\begin{array}{ll}40 & 44 \\
82 & 09\end{array}$ & 269 & 8,800 & 18 & 102,000 & 102,000 & 6 & F & 1936 & Corps of Engineers & $\mathrm{PA}$ \\
\hline $\begin{array}{l}\text { Mosqui to Creek } \\
\text { Mosquit to Creek }\end{array}$ & $03-0950$ OO & $\begin{array}{ll}41 & 18 \\
80 & 45\end{array}$ & 974 & 8,900 & $\mathrm{~b} 2,000$ & 104,100 & blo2,100 & 15 & CFNRW & 1944 & Corps of Engineers & $\mathrm{PA}$ \\
\hline $\begin{array}{l}\text { N1misilla } \\
\text { NImisilla Creek }\end{array}$ & | $303-116400$ & $\begin{array}{ll}41 & 00 \\
81 & 35\end{array}$ & 193 & 811 & 2,900 & 9,400 & 6,500 & 9 & $\mathrm{RW}$ & 1939 & State of Onio & - \\
\hline $\begin{array}{l}\text { O'Shaughnessy } \\
\text { Sc1oto River }\end{array}$ & | $\begin{array}{r}3 \mathrm{HB} \\
03-220500\end{array}$ & $\begin{array}{ll}40 & 09 \\
83 & 08\end{array}$ & 987 & 829 & 56 & 14,490 & 14,430 & $<05$ & M & 1924 & C1ty of Columbus & PA \\
\hline $\begin{array}{l}\text { Piedmont } \\
\text { Stiliwater Creek }\end{array}$ & | $03-125500$ & $\begin{array}{ll}40 & 11 \\
81 & 13\end{array}$ & 84 & 3,200 & 71 & 65,000 & 64,900 & 9 & $\mathrm{CFR}$ & 1937 & Corps of Engineers & PA \\
\hline $\begin{array}{l}\text { Pleasant Hill } \\
\text { Clear Fork }\end{array}$ & $03-133000$ & $\begin{array}{ll}40 & 37 \\
82 & 20\end{array}$ & 199 & 2,600 & 12 & 87,700 & 87,700 & 6 & CFR & 1938 & Corps of Engineers & PA \\
\hline $\begin{array}{l}\text { Rocky Fork } \\
\text { Rocky Fork }\end{array}$ & $03-232490$ & $\begin{array}{ll}39 & 11 \\
83 & 27\end{array}$ & 115 & 2,000 & o) & 34,100 & 34,100 & 4 & $\mathrm{R}$ & 1952 & State of On1o & - \\
\hline $\begin{array}{l}\text { Senecaville } \\
\text { Seneca Fork }\end{array}$ & $03-1410 \quad 00$ & $\begin{array}{ll}39 & 55 \\
81 & 26\end{array}$ & 121 & 5,170 & 2,200 & 88,500 & 86,300 & 10 & CFR & 1937 & Corps of Engineers & PA \\
\hline $\begin{array}{l}\text { Tappan } \\
\text { Little Stillwater Creek }\end{array}$ & $03-1280$ ODC & $\begin{array}{ll}40 & 22 \\
81 & 14\end{array}$ & 710 & 3,100 & 46 & 61,600 & 61,600 & 11 & $\mathrm{CF}$ & 1936 & Corps of Engineers & PA \\
\hline $\begin{array}{l}\text { Taylorsville } \\
\text { Great Miam River }\end{array}$ & $03-262990$ & $\begin{array}{ll}39 & 52 \\
84 & 10\end{array}$ & 1,155 & - & 0 & 186,000 & 186,000 & 3 & $\mathrm{~F}$ & 1922 & $\begin{array}{l}\text { M1am1 Conservancy } \\
\text { D1strict }\end{array}$ & - \\
\hline $\begin{array}{l}\text { West Fork Mill Creek } \\
\text { West Fork Mill Creek }\end{array}$ & $03-2565$ J JC & $\begin{array}{ll}39 & 16 \\
84 & 30\end{array}$ & 295 & 557 & 65 & 11,380 & 11,320 & 5 & FR & 1953 & Corps of Engineers & PA \\
\hline $\begin{array}{l}\text { W11ls Creek } \\
\text { W11ls Creek }\end{array}$ & $\mid \begin{array}{r}3 D G \\
03-1430\end{array}$ & $\begin{array}{ll}40 & 09 \\
81 & 51\end{array} \mid$ & 844 & 11,450 & 1,580 & 196,000 & 194,400 & 3 & $\mathrm{CF}$ & 1937 & Corps of Engineers & PA \\
\hline
\end{tabular}




\section{OKLAHOMA}

North Boggy Creek

Bluestem Lake

Canton

North Canadian River

Chisholm Tra1l Lake (Clear Creek Clear Creek

City of Hominy
Claremore Creek

Elm Creek

Elm Creek $c$ /

Canadian River
Canala

Fort Gibson

Neosho (Grand) River

Fort Supply
Wolf Creek

Foss

Wash1ta River

Great Salt Plains
Salt Fork Arkansas River

Greenleaf Lake

Greenleaf Creek

Heyburn

lecat Creek

Holdenville Lake
Beemore Creek

Hulah

Caney River

Lake Altus

North Fork Red River

Lake Carl Blackwell (St1llwater) ater Creek

Lake Chickasha (Spring Creek)

See footnotes at end of table

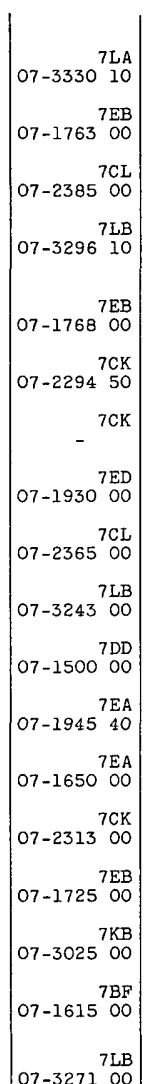

\begin{tabular}{|c|c|c|}
\hline $\begin{array}{ll}34 & 27 \\
96 & 05\end{array}$ & 176 & 5,900 \\
\hline $\begin{array}{ll}36 & 40 \\
96 & 20\end{array}$ & 440 & 70 \\
\hline $\begin{array}{ll}36 & 05 \\
98 & 36\end{array}$ & 7,600 & 15,500 \\
\hline $\begin{array}{ll}34 & 35 \\
97 & 50\end{array}$ & 201 & 7 \\
\hline $\begin{array}{ll}36 & 25 \\
96 & 25\end{array}$ & 56 & 30 \\
\hline $\begin{array}{ll}35 & 20 \\
97 & 21\end{array}$ & - & 2,900 \\
\hline $\begin{array}{ll}35 & 18 \\
95 & 22\end{array}$ & 47,520 & 48,000 \\
\hline $\begin{array}{ll}35 & 52 \\
95 & 14\end{array}$ & 12,492 & 51,000 \\
\hline $\begin{array}{ll}36 & 33 \\
99 & 34\end{array}$ & 1,494 & 5,500 \\
\hline $\begin{array}{ll}35 & 52 \\
99 & 11\end{array}$ & 1,496 & 13,140 \\
\hline $\begin{array}{ll}36 & 45 \\
98 & 08\end{array}$ & 3,194 & 27,500 \\
\hline $\begin{array}{ll}35 & 37 \\
95 & 10\end{array}$ & $\begin{array}{ll}81 & 2\end{array}$ & o \\
\hline $\begin{array}{ll}35 & 57 \\
96 & 18\end{array}$ & 123 & 4,000 \\
\hline $\begin{array}{ll}35 & 02 \\
96 & 72\end{array}$ & 90 & \\
\hline $\begin{array}{ll}36 & 56 \\
96 & 05\end{array}$ & 732 & 12,800 \\
\hline $\begin{array}{ll}34 & 54 \\
99 & 18\end{array}$ & 2,116 & 4,680 \\
\hline $\begin{array}{ll}36 & 08 \\
97 & 11\end{array}$ & $\begin{array}{ll}70 & 5\end{array}$ & 3,087 \\
\hline $\begin{array}{ll}35 & 08 \\
98 & 08\end{array}$ & $\begin{array}{ll}71 & 7\end{array}$ & 2,150 \\
\hline
\end{tabular}

$\begin{array}{r}584 \\ 0 \\ 63 \\ 0 \\ 0 \\ 3,000 \\ 897,000 \\ b 311,300 \\ 8,120 \\ 12,410 \\ 2,300 \\ 0 \\ -\quad 006 \\ 377 \\ \hline\end{array}$

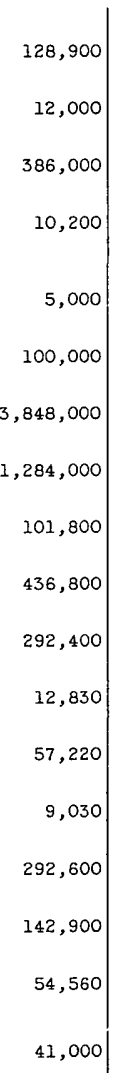

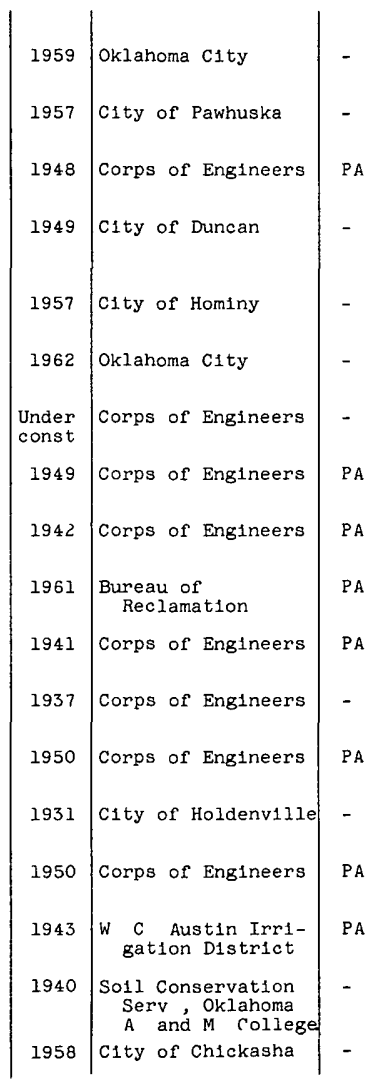

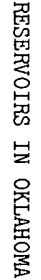


Table 1 --Reservo1rs in the Un1ted States completed or under construction as of January 1, 1963, having a usable capac1ty of 5,000 acre-feet or more--Cont1nued

\begin{tabular}{|c|c|c|c|c|c|c|c|c|c|c|c|c|}
\hline \multirow[b]{2}{*}{$\begin{array}{l}\text { Name of reservo1r } \\
\text { and } \\
\text { stream }\end{array}$} & \multicolumn{2}{|c|}{ Location } & \multirow{2}{*}{$\begin{array}{c}\text { Dra1nage } \\
\text { area } \\
\text { (square } \\
\text { m1les) }\end{array}$} & \multirow[b]{2}{*}{$\begin{array}{c}\text { Surface } \\
\text { area } \\
\text { (acres) }\end{array}$} & \multicolumn{3}{|c|}{ Storage in acre-feet } & \multirow[b]{2}{*}{$\begin{array}{c}\text { Storage } \\
\text { rat1o }\end{array}$} & \multirow[b]{2}{*}{ Use } & \multirow[b]{2}{*}{$\begin{array}{c}\text { Date } \\
\text { com- } \\
\text { pleted }\end{array}$} & \multirow[b]{2}{*}{$\begin{array}{l}\text { Owner } \\
\text { or } \\
\text { operator }\end{array}$} & \multirow{2}{*}{$\begin{array}{l}\text { Storage } \\
\text { records } \\
\text { pub- } \\
\text { lished }\end{array}$} \\
\hline & $\begin{array}{l}\text { Basin } \\
\text { 1ndex and } \\
\text { station no }\end{array}$ & $\begin{array}{l}\text { Lat1 tude } \\
\text { and } \\
\text { long1tude }\end{array}$ & & & Dead & Total & Usable & & & & & \\
\hline OKLAHOMA--Continued & & & & & & & & & & & & \\
\hline $\begin{array}{l}\text { Lake Duncan } \\
\text { Fitzpatrick Creek }\end{array}$ & $07-329620$ & $\begin{array}{ll}34 & 31 \\
97 & 49\end{array}$ & 11 & 395 & - & 5,783 & - & - & $\mathrm{MR}$ & 1937 & City of Duncan & - \\
\hline $\begin{array}{l}\text { Lake Ellsworth } \\
\text { East Cache Creek }\end{array}$ & $07-3089 \begin{array}{r}7 \mathrm{KC} \\
00\end{array}$ & $\begin{array}{ll}34 & 47 \\
98 & 22\end{array}$ & 246 & 3,500 & o & 95,000 & 95,000 & 19 & MR & 1962 & City of Lawton & - \\
\hline $\begin{array}{l}\text { Lake Elmer Thomas } \\
\text { L1ttle Medicine Creek }\end{array}$ & $07-3099 \begin{array}{r}7 \mathrm{KC} \\
00\end{array}$ & $\begin{array}{ll}34 & 44 \\
98 & 31\end{array}$ & 69 & 350 & 0 & 8,300 & - & - & $\mathrm{CR}$ & 1939 & $\begin{array}{l}\text { Fish and Wildlife } \\
\text { Service }\end{array}$ & - \\
\hline $\begin{array}{l}\text { Lake Hefner (Bluff Creek Dam) } \\
\text { North Canadian R1ver } \mathrm{c}\end{array}$ & $07-1595 \quad \begin{array}{r}7 \mathrm{BF} \\
10\end{array}$ & $\begin{array}{ll}35 & 35 \\
97 & 35\end{array}$ & - & 2,587 & 10,066 & 75,355 & 65,290 & - & MR & 1944 & Oklahoma C1ty & - \\
\hline $\begin{array}{l}\text { Lake Henryetta } \\
\text { Wolf Creek }\end{array}$ & $07-2440 \begin{array}{r}7 \mathrm{CL} \\
10\end{array}$ & $\begin{array}{ll}35 & 25 \\
95 & 55\end{array}$ & 225 & 616 & - & 8,624 & - & - & MR & 1928 & City of Henryetta & - \\
\hline $\begin{array}{l}\text { Lake Humphreys } \\
\text { Wildhorse Creek }\end{array}$ & $07-3296 \quad \begin{array}{r}7 \mathrm{LB} \\
00\end{array}$ & $\begin{array}{ll}34 & 35 \\
97 & 54\end{array}$ & 28 & 882 & 0 & 14,000 & 14,000 & 21 & MR & - & C1ty of Duncan & - \\
\hline $\begin{array}{l}\text { Lake Lawtonka } \\
\text { Medic1ne Creek }\end{array}$ & $07-3095 \begin{array}{r}7 \mathrm{KC} \\
00\end{array}$ & $\begin{array}{ll}34 & 44 \\
98 & 30\end{array}$ & 933 & 2,398 & 0 & 63,000 & 63,000 & 33 & MR & 1911 & C1ty of Lawton & - \\
\hline $\begin{array}{l}\text { Lake McAlester } \\
\text { Bull Creek }\end{array}$ & $07-2320^{7 \mathrm{CK}}$ & $\begin{array}{ll}35 & 01 \\
95 & 47\end{array}$ & 307 & 1,592 & - & 17,510 & - & - & MR & 1919 & City of McAlester & - \\
\hline $\begin{array}{l}\text { Lake Murray } \\
\text { Anadarche Creek }\end{array}$ & $07-3161 \quad 00$ & $\begin{array}{ll}34 & 02 \\
97 & 04\end{array}$ & 54 & 5,730 & - & 153,000 & - & - & $\mathrm{R}$ & 1937 & State of Oklahoma & - \\
\hline $\begin{array}{l}\text { Lake Okmulgee } \\
\text { Salt Creek }\end{array}$ & $07-2434$ 7CL & $\begin{array}{ll}35 & 37 \\
96 & 04\end{array}$ & 401 & 559 & - & 9,900 & - & - & $\mathrm{MR}$ & 1928 & City of okmulgee & - \\
\hline $\begin{array}{l}\text { Lake o' the Cherokees (Pensacola } \\
\text { Dam) } \\
\text { Neosho River }\end{array}$ & $\begin{array}{r}\text { 7ED } \\
07-1900 \quad 00\end{array}$ & $\begin{array}{ll}36 & 28 \\
95 & 02\end{array}$ & 10,298 & 59,500 & 180,000 & $2,197,000$ & $2,017,000$ & 4 & FPR & 1940 & $\begin{array}{l}\text { Grand River Dam } \\
\text { Authority }\end{array}$ & PA \\
\hline $\begin{array}{l}\text { Lake Overholser } \\
\text { North Canadian River }\end{array}$ & $07-2405$ 7CL & $\begin{array}{ll}35 & 29 \\
97 & 40\end{array}$ & 8,322 & 1,593 & 1,400 & 17,100 & 15,700 & 1 & MR & 1917 & Oklahoma C1ty & PA \\
\hline $\begin{array}{l}\text { Lake Ponca } \\
\text { Turkey Creek }\end{array}$ & $07-148150$ & $\begin{array}{ll}36 & 43 \\
97 & 01\end{array}$ & 28 & 805 & - & 14,140 & - & - & M & 1935 & Ponca C1ty & - \\
\hline $\begin{array}{l}\text { Lake Texoma (Denison Dam) * } \\
\text { Red R1ver }\end{array}$ & $07-331500$ & $\begin{array}{ll}33 & 49 \\
96 & 34\end{array}$ & 39,719 & 144,100 & $\mathrm{bl}, 106,000$ & $5,530,300$ & $\mathrm{~b} 4,424,300$ & 11 & FMPRW & 1943 & Corps of Eng1neers & PA \\
\hline $\begin{array}{l}\text { Pauls Valley City Lake } \\
\text { Washington Creek }\end{array}$ & $07-3286^{7 L} 00$ & $\begin{array}{ll}34 & 45 \\
97 & 14\end{array}$ & 233 & 750 & 0 & 8,500 & 8,500 & 10 & MR & 1954 & $\begin{array}{l}\text { C1ty of Pauls } \\
\text { Valley }\end{array}$ & - \\
\hline
\end{tabular}




\begin{tabular}{|c|c|}
\hline $\begin{array}{l}\text { Perry Lake } \\
\text { Cow Creek }\end{array}$ & $\begin{array}{rl}7 D E \\
07-1526 & 00\end{array}$ \\
\hline $\begin{array}{l}\text { Public Service Co No } 3 \\
\text { Leaper Creek c/ }\end{array}$ & $07-3263^{7 L B}$ \\
\hline $\begin{array}{l}\text { Shawnee Lake } \\
\text { South Fork Deer Creek }\end{array}$ & $07-2416$ PCL \\
\hline $\begin{array}{l}\text { Shawnee No }{ }^{2} \\
\text { South Deer Creek (West Fork) }\end{array}$ & $07-2415^{7 C L} 90$ \\
\hline $\begin{array}{l}\text { Shell Creek Lake } \\
\text { Shell Creek }\end{array}$ & $07-164390$ \\
\hline $\begin{array}{l}\text { Spavinaw } \\
\text { Spavinaw Creek }\end{array}$ & $07-191350$ \\
\hline $\begin{array}{l}\text { Tenkiller Ferry } \\
\text { Illinols River }\end{array}$ & $07-197500$ \\
\hline $\begin{array}{l}\text { Upper Spavinaw Lake (Eucha) } \\
\text { Spavinaw Creek }\end{array}$ & 7ED \\
\hline $\begin{array}{l}\text { Wister } \\
\text { Poteau River }\end{array}$ & $07-2480^{7 \mathrm{FA}} 00$ \\
\hline $\begin{array}{l}\text { Yahola } \\
\text { Spavinaw Creek } \underline{c} / \\
\text { OREGoN }\end{array}$ & $07-1779$ PEB \\
\hline $\begin{array}{l}\text { Agency Valley (Beulah) } \\
\text { North Fork Malheur River }\end{array}$ & $13-217000$ \\
\hline $\begin{array}{l}\text { Antelope } \\
\text { Jordan Creek } c /\end{array}$ & $\begin{array}{r}13 D E \\
13-179100\end{array}$ \\
\hline $\begin{array}{l}\text { B1g Cliff } \\
\text { North Sant1am River }\end{array}$ & $14-180600$ \\
\hline $\begin{array}{l}\text { Bonneville } * \\
\text { Columb1a River }\end{array}$ & $\begin{array}{r}14 \mathrm{BG} \\
14-128850\end{array}$ \\
\hline $\begin{array}{l}\text { Brownlee ** } \\
\text { Snake River }\end{array}$ & $\begin{array}{r}13 \mathrm{DK} \\
13-289700\end{array}$ \\
\hline $\begin{array}{l}\text { Buil Run Lake } \\
\text { Bujl Run Lake }\end{array}$ & $14-138600$ \\
\hline $\begin{array}{l}\text { Bull Run No } 2 \\
\text { Bull Run River }\end{array}$ & $14-139900$ \\
\hline $\begin{array}{l}\text { Clear Lake (Wasco Dam) } \\
\text { Clear Lake }\end{array}$ & $\begin{array}{r}14 \mathrm{BK} \\
14-097400\end{array}$ \\
\hline
\end{tabular}

\begin{tabular}{|c|c|c|c|c|c|c|c|}
\hline $\begin{array}{ll}36 & 15 \\
97 & 20\end{array}$ & 14 & 400 & 50 & 5,700 & 5,650 & 15 & MR \\
\hline $\begin{array}{ll}35 & 07 \\
98 & 20\end{array}$ & - & 170 & 153 & 21,047 & 10,894 & - & W \\
\hline $\begin{array}{ll}35 & 21 \\
97 & 04\end{array}$ & 220 & 1,370 & - & 23,900 & - & - & $\mathrm{MR}$ \\
\hline $\begin{array}{ll}35 & 21 \\
97 & 04\end{array}$ & 11 & 800 & 535 & 11,400 & 10,860 & - & MR \\
\hline $\begin{array}{ll}36 & 11 \\
96 & 11\end{array}$ & 370 & 620 & o) & 15,300 & 15,300 & - & MW \\
\hline $\begin{array}{ll}36 & 23 \\
95 & 03\end{array}$ & 390 & 1,638 & 9,500 & 30,510 & 21,000 & 2 & $\mathrm{MR}$ \\
\hline $\begin{array}{ll}35 & 36 \\
95 & 03\end{array}$ & 1,610 & 20,800 & 500 & $1,231,000$ & $1,230,500$ & 11 & FPR \\
\hline $\begin{array}{ll}36 & 25 \\
94 & 55\end{array}$ & 358 & 3,190 & o & 80,000 & 80,000 & 3 & $M$ \\
\hline $\begin{array}{ll}34 & 56 \\
94 & 43\end{array}$ & 993 & 23,500 & 0 & 429,600 & 429,600 & 5 & FR \\
\hline $\begin{array}{ll}36 & 13 \\
95 & 56\end{array}$ & - & 425 & 0 & 6,450 & 6,450 & - & M \\
\hline $\begin{array}{r}4355 \\
11809\end{array}$ & $\mathrm{a} 440$ & 1,900 & 0 & 59,920 & 59,920 & 6 & I \\
\hline $\begin{array}{r}4255 \\
11710\end{array}$ & - & 2,880 & o & 36,500 & 36,500 & - & I \\
\hline $\begin{array}{r}4445 \\
122 \quad 17\end{array}$ & 452 & 100 & b3,500 & 5,930 & $b 2,430$ & $<05$ & $\mathrm{P}$ \\
\hline $\begin{array}{r}4549 \\
121 \quad 57\end{array}$ & 240,000 & 20,400 & 450,000 & b719,000 & b87,000 & $<05$ & NP \\
\hline $\begin{array}{r}4450 \\
11654\end{array}$ & 72,590 & 15,100 & 426,700 & $1,426,700$ & $1,000,000$ & - & NP \\
\hline $\begin{array}{r}4528 \\
12151\end{array}$ & a 4 & - & - & 10,000 & - & - & M \\
\hline $\begin{array}{r}45 \quad 27 \\
122 \quad 09\end{array}$ & 102 & 418 & 0 & 21,000 & 21,000 & $<05$ & MP \\
\hline $\begin{array}{r}4510 \\
12141\end{array}$ & $a 8$ & 557 & 1,200 & 13,100 & 11,900 & 11 & IR \\
\hline
\end{tabular}

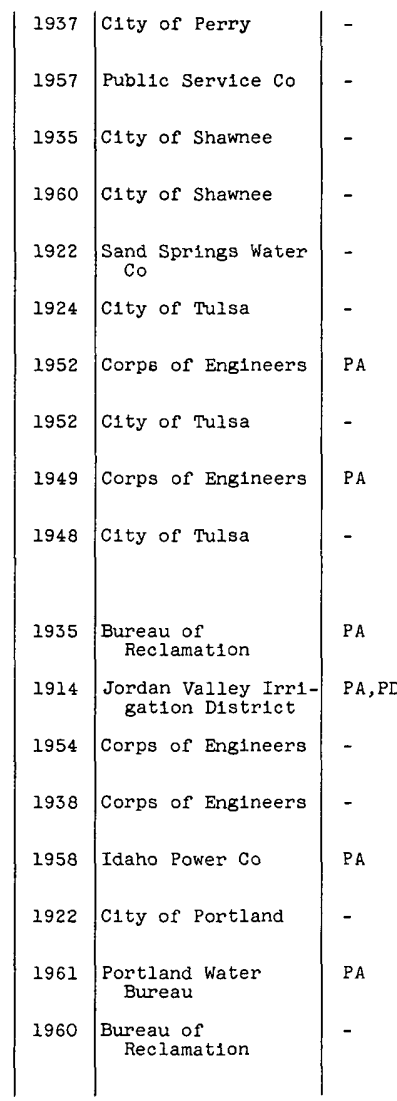




\begin{tabular}{|c|c|c|c|c|c|c|c|c|c|c|c|c|}
\hline \multirow[b]{2}{*}{$\begin{array}{l}\text { Name of reservo1r } \\
\text { and } \\
\text { stream }\end{array}$} & \multicolumn{2}{|c|}{ Location } & \multirow{2}{*}{$\begin{array}{l}\text { Dralnage } \\
\text { area } \\
\text { (square } \\
\text { m1les) }\end{array}$} & \multirow[b]{2}{*}{$\begin{array}{c}\text { Surface } \\
\text { area } \\
\text { (acres) }\end{array}$} & \multicolumn{3}{|c|}{ Storage in acre-feet } & \multirow[b]{2}{*}{$\mid \begin{array}{c}\text { Storage } \\
\text { rat1o }\end{array}$} & \multirow[b]{2}{*}{ Use } & \multirow[b]{2}{*}{$\begin{array}{l}\text { Date } \\
\text { com- } \\
\text { pleted }\end{array}$} & \multirow[b]{2}{*}{$\begin{array}{l}\text { Owner } \\
\text { or } \\
\text { operator }\end{array}$} & \multirow{2}{*}{$\begin{array}{l}\text { Storage } \\
\text { records } \\
\text { pub- } \\
\text { 11shed }\end{array}$} \\
\hline & $\begin{array}{c}\text { Basin } \\
\text { 1ndex and } \\
\text { station no }\end{array}$ & $\begin{array}{l}\text { Latitude } \\
\text { and } \\
\text { longitude }\end{array}$ & & & Dead & Total & Usable & & & & & \\
\hline OREGON--Continued & & & & & & & & & & & & \\
\hline $\begin{array}{l}\text { Cold Springs } \\
\text { Umatilla River } \mathrm{c}\end{array}$ & $\begin{array}{r}14 \mathrm{BA} \\
14-019150\end{array}$ & $\begin{array}{rr}45 & 52 \\
119 & 10\end{array}$ & - & 1,550 & - & - & 50,000 & - & I & 1908 & $\begin{array}{l}\text { Bureau of } \\
\text { Reclamation }\end{array}$ & - \\
\hline $\begin{array}{l}\text { Cottage Grove } \\
\text { Coast Fork Willamette River }\end{array}$ & $14-153000$ & $\begin{array}{rr}43 & 43 \\
123 & 03\end{array}$ & 104 & 1,160 & 0 & 33,000 & 33,000 & 02 & FINP & 1942 & Corps of Englneers & PA \\
\hline $\begin{array}{l}\text { Cottonwood } \\
\text { Cottonwood Creek }\end{array}$ & $11-340000$ & $\begin{array}{rr}42 & 14 \\
120 & 30\end{array}$ & 30 & 393 & 0 & 4,160 & 4,160 & 3 & I & 1923 & $\begin{array}{l}\text { Lakeview Water } \\
\text { Users, Inc }\end{array}$ & $\mathrm{PA}$ \\
\hline $\begin{array}{l}\text { Cow Lake (Upper) } \\
\text { Cow Creek }\end{array}$ & $\begin{array}{r}13 \mathrm{DE} \\
13-1794 \quad 00\end{array}$ & $\begin{array}{rr}43 & 06 \\
117 & 20\end{array}$ & - & - & - & - & a5,, 000 & - & I & 1929 & $\begin{array}{l}\text { Jordan Valley } \\
\text { Cattle Co }\end{array}$ & - \\
\hline $\begin{array}{l}\text { Crane Pralrie } \\
\text { Deschutes River }\end{array}$ & $14-053500$ & $\begin{array}{rr}43 & 45 \\
121 & 47\end{array}$ & 254 & 4,940 & o & 55,340 & 55,340 & 4 & I & 1940 & $\begin{array}{l}\text { Central Oreg and } \\
\text { Arnold Irrig Dist. } \\
\text { and Crook County } \\
\text { Improv Dist No } 1\end{array}$ & PA \\
\hline $\begin{array}{l}\text { Crescent Lake } \\
\text { Crescent Creek }\end{array}$ & $\begin{array}{r}14 \mathrm{BH} \\
14-059500\end{array}$ & $\begin{array}{rr}43 & 30 \\
121 & 58\end{array}$ & 607 & - & - & - & 117,200 & 28 & I & 1922 & $\begin{array}{l}\text { Deschutes Co Munic- } \\
\text { 1pal Improv Dist }\end{array}$ & $\mathrm{PA}$ \\
\hline $\begin{array}{l}\text { Detroit } \\
\quad \text { North Santiam River }\end{array}$ & $\begin{array}{r}14 \mathrm{AE} \\
14-180500\end{array}$ & $\begin{array}{rr}44 & 43 \\
122 & 15\end{array}$ & 437 & 3,580 & 115,000 & 454,900 & 339,900 & 2 & FIMNPR & 1953 & Corps of Englneers & PA \\
\hline $\begin{array}{l}\text { Dexter } \\
\text { MIddle Fork Willamette River }\end{array}$ & $\begin{array}{r}14 \mathrm{AB} \\
14-149100\end{array}$ & $\begin{array}{rr}43 & 55 \\
122 & 48\end{array}$ & 996 & 1,025 & b22,700 & 27,500 & 4,800 & $<05$ & $P$ & 1955 & Corps of Eng1neers & - \\
\hline $\begin{array}{l}\text { Dorena } \\
\text { Row River }\end{array}$ & $\begin{array}{r}14 \mathrm{AA} \\
14-155000\end{array}$ & $\begin{array}{rr}43 & 47 \\
122 & 57\end{array}$ & 265 & 1,840 & b7, 000 & 77,500 & b10,500 & 1 & FINR & 1949 & Corps of Engineers & PA \\
\hline $\begin{array}{l}\text { Drews Creek } \\
\text { Drews Creek }\end{array}$ & $\begin{array}{r}11 \mathrm{BB} \\
11-3385 \quad 00\end{array}$ & $\begin{array}{rr}42 & 07 \\
120 & 37\end{array}$ & 211 & 4,540 & 408 & 62,500 & 62,100 & 14 & I & 1912 & $\begin{array}{l}\text { Lakeview Water } \\
\text { Users, Inc }\end{array}$ & $P A, P D$ \\
\hline $\begin{array}{l}\text { Emigrant Lake } \\
\text { Emigrant Creek }\end{array}$ & $\begin{array}{r}14 C D \\
14-349000\end{array}$ & $\begin{array}{rr}42 & 10 \\
122 & 36\end{array}$ & 639 & - & 1,530 & 40,500 & 39,000 & 22 & FI & 1924 & $\begin{array}{l}\text { Talent Irrigation } \\
\text { District }\end{array}$ & $\mathrm{PA}$ \\
\hline $\begin{array}{l}\text { Fern Ridge } \\
\text { Long Tom River and Coyote Creek }\end{array}$ & $\begin{array}{r}14 \mathrm{AC} \\
14-1680 \quad 00\end{array}$ & $\begin{array}{rr}44 & 07 \\
123 & 18\end{array}$ & 252 & 9,360 & 7,000 & 101,200 & 94,200 & 2 & FINR & 1941 & Corps of Engineers & PA \\
\hline $\begin{array}{l}\text { F1sh Lake } \\
\text { North Fork Little Butte Creek }\end{array}$ & $\begin{array}{r}14 \mathrm{CD} \\
14-342000\end{array}$ & $\begin{array}{rr}42 & 23 \\
122 & 21\end{array}$ & 201 & 407 & 0 & 8,020 & 8,020 & 3 & I & 1915 & $\begin{array}{l}\text { Medford Irrigation } \\
\text { District }\end{array}$ & PA \\
\hline $\begin{array}{l}\text { Fourmile Lake } \\
\text { Fourm1le Creek }\end{array}$ & $\begin{array}{r}11 \mathrm{AB} \\
11-504500\end{array}$ & $\begin{array}{rr}42 & 27 \\
122 & 15\end{array}$ & 106 & 900 & of & $\mathrm{d} 13,330$ & $d 13,330$ & 13 & I & 1956 & $\left\{\begin{array}{l}\text { Medford Irrigation } \\
\text { District }\end{array}\right.$ & PA \\
\hline $\begin{array}{l}\text { Gerber } \\
\text { Miller Creek }\end{array}$ & 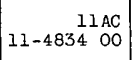 & $\begin{array}{rr}42 & 12 \\
121 & 08\end{array}$ & 220 & 3,845 & 0 & 94,270 & 94,270 & 24 & FI & 1925 & $\begin{array}{l}\text { Bureau of } \\
\text { Reclamation }\end{array}$ & - \\
\hline
\end{tabular}


Greaser Lake c/

Hart Lake

Twentymile, Dee'p, and Honey
Creeks c/

Hays tack

Creek c/

Hills Creek

Middle Fork Willamette River

Howard Prairie Lake

Orizzly Creek (Beaver Creek)

Hyatt

Keene Creek

Lake Ben Morrow

ake cel110*

Lake Celilo *
Columbia Ríver

Lake Owyhee
Owyhee River

Lake Wallula (McNary Dam) *

Columbia Rive

Lemolo Lake

North Umpqua River

Lookout Point
M1ddle Fork Willamette River McKay

McKay Creek

North Fork

ackamas River

Ochoco

Ochoco Creek

oxbow *

River

Prineville
Crooked River

Riddle Creek (Round Barn)

Rock Creek (Upper)

Rock Creek

See footnotes at end of table

\begin{tabular}{|c|c|c|}
\hline $\mid \begin{array}{r}10 \mathrm{AE} \\
10-366100\end{array}$ & \begin{tabular}{rr|}
42 & 21 \\
119 & 49
\end{tabular} & - \\
\hline $\begin{array}{r}10 \mathrm{AEE} \\
10-379000\end{array}$ & 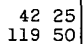 & - \\
\hline $\begin{array}{r}14 \mathrm{BH} \\
14-069200\end{array}$ & $\begin{array}{rr}44 & 29 \\
121 & 09\end{array}$ & - \\
\hline $\begin{array}{r}14 \mathrm{AB} \\
14-145100\end{array}$ & $\begin{array}{rr}43 & 42 \\
122 & 25\end{array}$ & 389 \\
\hline $\begin{array}{r}11 \mathrm{AB} \\
11-512900\end{array}$ & $\begin{array}{rr}42 & 13 \\
122 & 33\end{array}$ & 276 \\
\hline $\begin{array}{r}11 \mathrm{AE} \\
11-514000\end{array}$ & $\begin{array}{rr}42 & 10 \\
122 & 28\end{array}$ & 117 \\
\hline $\begin{array}{r}14 \mathrm{BO} \\
14-13900 \mathrm{O}\end{array}$ & $\begin{array}{rr}45 & 29 \\
122 & 05\end{array}$ & 74 \\
\hline $\begin{array}{r}14 \mathrm{BG} \\
14-103900\end{array}$ & $\begin{array}{rr}45 & 37 \\
121 & 07\end{array}$ & 237,000 \\
\hline $\begin{array}{r}13 D E \\
13-182500\end{array}$ & $\begin{array}{rr}43 & 38 \\
217 & 15\end{array}$ & 11,160 \\
\hline $\begin{array}{r}14 \mathrm{BA} \\
14-019190\end{array}$ & $\begin{array}{rr}45 & 56 \\
119 & 18\end{array}$ & 214,000 \\
\hline $\begin{array}{r}14 \mathrm{CB} \\
14-313000\end{array}$ & $\begin{array}{rr}43 & 20 \\
122 & 12\end{array}$ & 170 \\
\hline $14-1490^{14} \mathrm{AB}$ & $\begin{array}{rr}43 & 55 \\
122 & 45\end{array}$ & 991 \\
\hline $\begin{array}{r}14 \mathrm{BC} \\
14-023000\end{array}$ & $\begin{array}{rr}45 & 36 \\
118 & 48\end{array}$ & 186 \\
\hline $\begin{array}{r}14 \mathrm{AG} \\
14-2098 \quad 00\end{array}$ & $\begin{array}{rr}45 & 15 \\
122 & 16\end{array}$ & 665 \\
\hline $\begin{array}{r}14 \mathrm{BJ} \\
14-085100\end{array}$ & $\begin{array}{rr}44 & 18 \\
120 & 44\end{array}$ & 300 \\
\hline$-13 \mathrm{DK}$ & $\begin{array}{rr}44 & 58 \\
116 & 51\end{array}$ & 73,150 \\
\hline $\begin{array}{r}14 \mathrm{BJ} \\
14-080400\end{array}$ & $\begin{array}{rr}44 & 07 \\
120 & 47\end{array}$ & a2, 700 \\
\hline $\begin{array}{r}10 \mathrm{AE} \\
10-401100\end{array}$ & \begin{tabular}{rr|}
43 & 10 \\
118 & 39
\end{tabular} & - \\
\hline$\left|\begin{array}{r}10 A E \\
10-395700\end{array}\right|$ & $\begin{array}{rr}42 & 42 \\
119 & 18\end{array} \mid$ & - \\
\hline
\end{tabular}

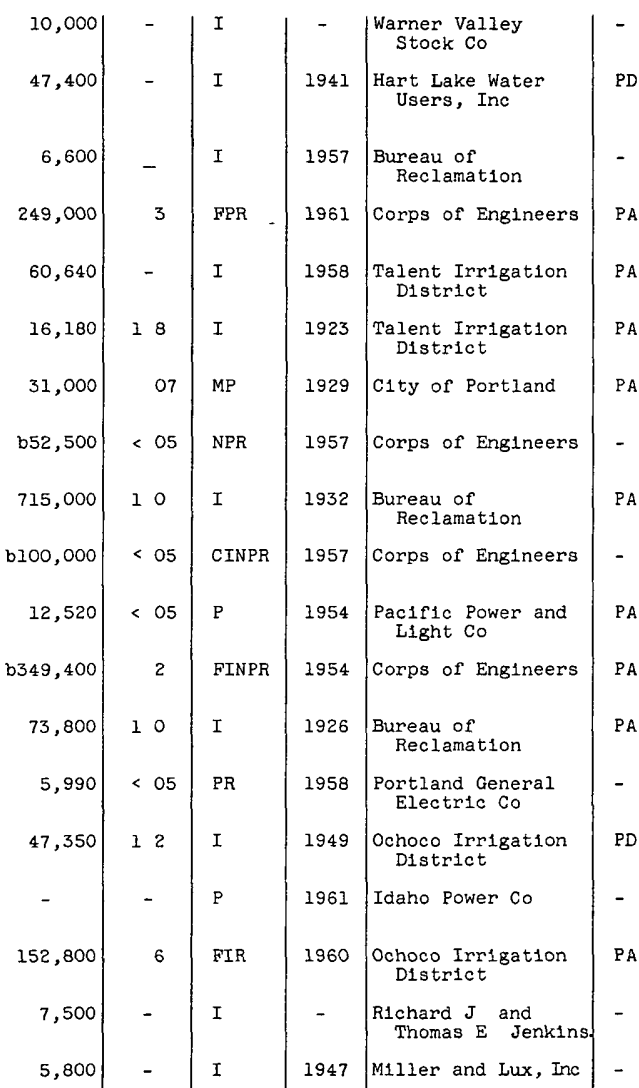

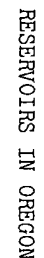


Table 1 --Reservo1rs in the United States completed or under construction as of January 1, 1963, having a usable capac1ty of 5,000 acre-feet or more--Continued

\begin{tabular}{|c|c|c|c|c|c|c|c|c|c|c|c|c|}
\hline \multirow[b]{2}{*}{$\begin{array}{l}\text { Name of reservo1r } \\
\text { and } \\
\text { stream }\end{array}$} & \multicolumn{2}{|c|}{ Location } & \multirow{2}{*}{$\begin{array}{l}\text { Drainage } \\
\text { area } \\
\text { (square } \\
\text { miles) }\end{array}$} & \multirow[b]{2}{*}{$\begin{array}{c}\text { Surface } \\
\text { area } \\
\text { (acres) }\end{array}$} & \multicolumn{3}{|c|}{ Storage in acre-feet } & \multirow[b]{2}{*}{$\begin{array}{c}\text { Storage } \\
\text { rat10 }\end{array}$} & \multirow[b]{2}{*}{ Use } & \multirow[b]{2}{*}{$\mid \begin{array}{c}\text { Date } \\
\text { com- } \\
\text { pleted }\end{array}$} & \multirow[b]{2}{*}{$\begin{array}{l}\text { Owner } \\
\text { or } \\
\text { operator }\end{array}$} & \multirow{2}{*}{$\begin{array}{l}\text { Storage } \\
\text { records } \\
\text { pub- } \\
\text { lished }\end{array}$} \\
\hline & \begin{tabular}{|l} 
Bas1n \\
index and \\
station no
\end{tabular} & $\begin{array}{l}\text { Lat1tude } \\
\text { and } \\
\text { long1tude }\end{array}$ & & & Dead & Total & Usable & & & & & \\
\hline \multirow{2}{*}{ OREGON--Cont1nued } & & & & & & & & & & & & \\
\hline & $\begin{array}{r}10 \mathrm{AA} \\
10-405400\end{array}$ & \begin{tabular}{rr|}
43 & 25 \\
119 & 24
\end{tabular} & 307 & 630 & of & 8,240 & 8,240 & - & $I$ & 1952 & $J \quad V$ Moon, et al & - \\
\hline $\begin{array}{l}\text { Thief Valley } \\
\text { Powder River }\end{array}$ & $\begin{array}{r}13 D L \\
13-285000\end{array}$ & $\begin{array}{rr}45 & 00 \\
117 & 47\end{array}$ & 826 & 750 & 170 & 27,570 & 17,400 & - & I & 1932 & $\begin{array}{l}\text { Lower Powder R1ver } \\
\text { Irrigation D1st }\end{array}$ & - \\
\hline $\begin{array}{l}\text { Thompson Valley } \\
\text { Silver Creek }\end{array}$ & $\begin{array}{r}10 \mathrm{AC} \\
10-388800\end{array}$ & $\begin{array}{rr}42 & 58 \\
121 & 05\end{array}$ & - & 1,900 & 0 & 17,400 & 17,400 & - & I & 1923 & $\begin{array}{l}\text { S1lver Lake Irr1- } \\
\text { gat1on D1strict }\end{array}$ & - \\
\hline $\begin{array}{l}\text { T1mothy Lake } \\
\text { Oak Grove Fork }\end{array}$ & $\begin{array}{r}14 \mathrm{AG} \\
14-208600\end{array}$ & $\begin{array}{rr}45 & 07 \\
121 & 48\end{array}$ & 535 & 1,401 & $b 4,060$ & 65,710 & $b 61,650$ & 07 & $P$ & 1956 & $\begin{array}{l}\text { Portland General } \\
\text { Electric Co }\end{array}$ & PA \\
\hline $\begin{array}{l}\text { Unity } \\
\text { Burnt River }\end{array}$ & $13-272500$ & \begin{tabular}{rr|}
44 & 30 \\
118 & 11
\end{tabular} & 309 & 930 & 600 & 25,820 & 25,220 & 4 & I & 1938 & $\begin{array}{l}\text { Burnt R1ver Irri- } \\
\text { gation D1strict }\end{array}$ & PA \\
\hline $\begin{array}{l}\text { Upper Klamath Lake (Link River Dam) } \\
\text { Klamath RIver }\end{array}$ & $\begin{array}{r}11 \mathrm{AB} \\
11-507000\end{array}$ & $\begin{array}{rr}42 & 15 \\
121 & 48\end{array}$ & 3,810 & 90,100 & - & $\mathrm{a} 873,000$ & 584,000 & 5 & FIP & 1921 & $\begin{array}{l}\text { Bureau of } \\
\text { Reclamation }\end{array}$ & $\mathrm{PA}$ \\
\hline $\begin{array}{l}\text { Wallowa Lake } \\
\text { Wallowa R1ver }\end{array}$ & $\begin{array}{r}13 D O \\
13-326000\end{array}$ & \begin{tabular}{rr|}
45 & 20 \\
217 & 13
\end{tabular} & 508 & 1,670 & 25,300 & 42,750 & $b 37,450$ & 4 & I & 1929 & $\begin{array}{l}\text { Assoc1ation D1tch } \\
\text { Co }\end{array}$ & PA \\
\hline $\begin{array}{l}\text { Warmsprings } \\
\text { M1ddle Fork Malheur R1ver }\end{array}$ & $\begin{array}{r}13 \mathrm{DH} \\
13-214500\end{array}$ & $\begin{array}{rr}43 & 35 \\
118 & 12\end{array}$ & 1,100 & 4,600 & 1,400 & d191,000 & $\mathrm{d} 189,600$ & 16 & I & 1919 & $\begin{array}{l}\text { Bureau of Reclama- } \\
\text { t1on, Warm } \\
\text { Springs Irriga- } \\
\text { t1on D1str1ct }\end{array}$ & PA \\
\hline $\begin{array}{l}\text { Wicklup } \\
\text { Deschutes River }\end{array}$ & $\begin{array}{r}14 \mathrm{BH} \\
14-0560 \mathrm{OO}\end{array}$ & \begin{tabular}{rr|}
43 & 41 \\
121 & 41
\end{tabular} & 482 & 10,640 & 0 & 182,100 & 182,100 & 3 & IR & 1942 & $\begin{array}{l}\text { Bureau of } \\
\text { Reclamation }\end{array}$ & PA \\
\hline $\begin{array}{l}\text { W11low Creek } \\
\text { W11low Creek }\end{array}$ & $\begin{array}{r}14 \mathrm{CD} \\
14-335240\end{array}$ & \begin{tabular}{rr|}
42 & 29 \\
122 & 27
\end{tabular} & 21 & 322 & 0 & 7,500 & 7,500 & - & IM & 1952 & C1ty of Medford & - \\
\hline $\begin{array}{l}\text { Willow Creek No } 3 \\
\text { W11low Creek }\end{array}$ & $13-230000$ & \begin{tabular}{rr|}
44 & 20 \\
117 & 40
\end{tabular} & 275 & 1,240 & 0 & 49,000 & b13,900 & - & I & 1911 & Orchards Water co & - \\
\hline \multicolumn{13}{|l|}{ PENNSYLVANIA } \\
\hline $\begin{array}{l}\text { Beaver Run } \\
\text { Beaver Run }\end{array}$ & $\begin{array}{r}3 \mathrm{AL} \\
03-048400\end{array}$ & $\begin{array}{ll}40 & 31 \\
79 & 33\end{array}$ & 432 & 1,125 & 0 . & 28,600 & 28,600 & 6 & M & 1951 & $\begin{array}{l}\text { Municipal Authority } \\
\text { of Westmoreland } \\
\text { County }\end{array}$ & - \\
\hline $\begin{array}{l}\text { Conemaugh R1ver } \\
\text { Conemaugh R1ver }\end{array}$ & $03-043500$ & $\begin{array}{ll}40 & 28 \\
79 & 22\end{array}$ & 1,351 & 6,820 & 400 & 274,000 & 273,600 & 16 & FR & 1953 & Corps of Englneers & $\mathrm{PA}$ \\
\hline $\begin{array}{l}\text { Crooked Creek } \\
\text { Crooked Creek }\end{array}$ & - $03-038500$ & $\begin{array}{ll}40 & 43 \\
79 & 30\end{array}$ & 277 & 1,940 & o) & 93,900 & 93,900 & 3 & $\mathrm{FR}$ & 1940 & Corps of Engineers & $\mathrm{PA}, \mathrm{PF}$ \\
\hline
\end{tabular}


DeHart (formerly Clark Creek)

Clark Creek

East Branch Clarion River

F1rst Fork Sinnemahoning Creek

First Fork Sinnemahoning Creek

Francis E Walter

Geist (formerly Springton)

General Edgar Jadwin

Dyberry Creek

Glendale
Beaverdam Run

Hol twood

Susquehanna River

Huntsville

Tobys Creek

Indian Rock

West Branch Codorus Creek

Kettle Creek (Alvin $R$ Bush Dam)

Lake Scranton
Stafford Meadow Brook

Lake Wallenpaupack

Little Pine Creek

Little PIne Creek

Loyalhanna

Loyalhanna Creek

Mahoning

Mahoning Creek

Ontelaunee

Ma1den Creek

Penn Forest

ld Creek

P1kes Creek

See footnotes at end of table

\begin{tabular}{|c|c|c|c|}
\hline $\begin{array}{r}1 \mathrm{PE} \\
01-5684 \stackrel{00}{0}\end{array}$ & $\begin{array}{ll}40 & 28 \\
76 & 45\end{array}$ & $\begin{array}{ll}21 & 7\end{array}$ & - \\
\hline $03-0270$ o 00 & $\begin{array}{ll}41 & 34 \\
78 & 36\end{array}$ & $\begin{array}{ll}72 & 7\end{array}$ & 1,370 \\
\hline $\begin{array}{r}100 \\
01-543700\end{array}$ & $\begin{array}{ll}41 & 24 \\
78 & 01\end{array}$ & 243 & 1,450 \\
\hline $01-4477_{80}^{1 \mathrm{LH}}$ & $\begin{array}{ll}41 & 07 \\
75 & 43\end{array} \mid$ & 289 & - \\
\hline $01-4759$ OOK & $\begin{array}{ll}39 & 57 \\
75 & 24\end{array}$ & $\begin{array}{lll}21 & 3\end{array}$ & 391 \\
\hline $\begin{array}{r}\text { ILE } \\
01-4294 \text { DO }\end{array}$ & $\begin{array}{ll}41 & 37 \\
75 & 16\end{array}$ & $\begin{array}{ll}64 & 5\end{array}$ & - \\
\hline $01-5413^{10 \mathrm{~A}}$ & $\begin{array}{ll}40 & 42 \\
78 & 32\end{array}$ & $\begin{array}{ll}41 & 9\end{array}$ & 1,600 \\
\hline $01-5769^{1 P H}$ & $\begin{array}{ll}39 & 50 \\
76 & 20\end{array}$ & 26,794 & $\mathrm{~d} 2,400$ \\
\hline $01-536900$ & $\begin{array}{ll}41 & 18 \\
75 & 58\end{array}$ & $\begin{array}{ll}8 & 4\end{array}$ & 381 \\
\hline $\begin{array}{r}1 P G \\
01-5749 \text { OO }\end{array}$ & $\begin{array}{ll}39 & 55 \\
76 & 45\end{array}$ & $93 \quad 7$ & 2,080 \\
\hline $\begin{array}{r}10 D \\
01-5448 \text { OD }\end{array}$ & $\begin{array}{ll}41 & 22 \\
77 & 55\end{array}$ & 226 & - \\
\hline $01-535100$ & $\begin{array}{ll}41 & 23 \\
75 & 38\end{array}$ & $\begin{array}{ll}6 & 0\end{array}$ & 225 \\
\hline $01-4317 \frac{1 L D}{00}$ & $\begin{array}{ll}41 & 28 \\
75 & 11\end{array}$ & 228 & 6,200 \\
\hline $01-5495^{1 O G}$ & $\begin{array}{ll}41 & 21 \\
77 & 21\end{array}$ & 165 & 634 \\
\hline $\begin{array}{r}3 \mathrm{AL} \\
03-0465 \mathrm{OO}\end{array}$ & $\begin{array}{ll}40 & 27 \\
79 & 27\end{array} \mid$ & 290 & 3,280 \\
\hline $\begin{array}{r}3 \mathrm{AJ} \\
03-035500\end{array}$ & $\begin{array}{ll}40 & 55 \\
79 & 17\end{array}$ & 340 & 2,370 \\
\hline $\begin{array}{r}1 M H \\
01-470759\end{array}$ & $\begin{array}{ll}40 & 27 \\
75 & 56\end{array}$ & 192 & 1,082 \\
\hline $\begin{array}{r}1 \mathrm{LJ} \\
01-4494 \quad 00\end{array}$ & $\begin{array}{ll}40 & 56 \\
75 & 34\end{array}$ & 165 & 462 \\
\hline $\begin{array}{r}1 \mathrm{NM} \\
01-5376 \text { OO }\end{array}$ & $\begin{array}{ll}41 & 16 \\
76 & 03\end{array}$ & $\begin{array}{ll}11 & 1\end{array}$ & 399 \\
\hline
\end{tabular}

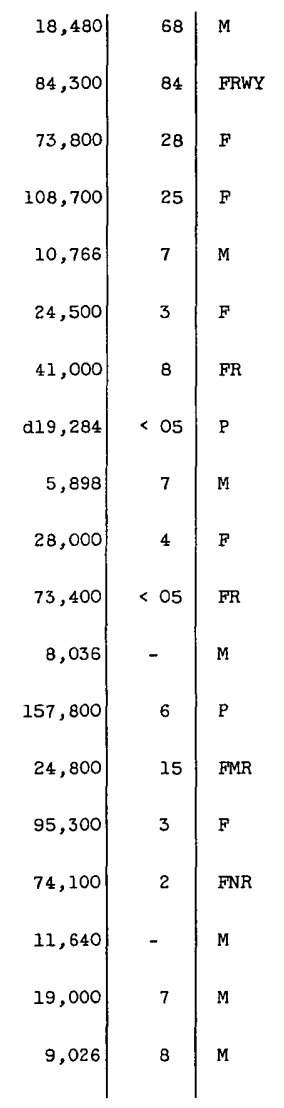

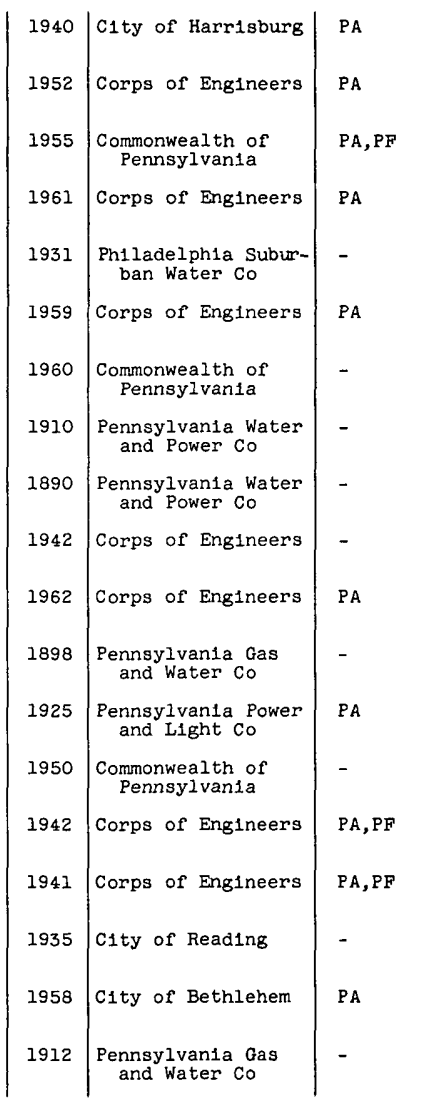




\begin{tabular}{|c|c|c|c|c|c|c|c|c|c|c|c|c|}
\hline \multirow[b]{2}{*}{$\begin{array}{l}\text { Name of reservolr } \\
\text { and } \\
\text { stream }\end{array}$} & \multicolumn{2}{|c|}{ Location } & \multirow{2}{*}{$\begin{array}{l}\text { Drainage } \\
\text { area } \\
\text { (square } \\
\text { m1les) }\end{array}$} & \multirow[b]{2}{*}{$\begin{array}{c}\text { Surface } \\
\text { area } \\
\text { (acres) }\end{array}$} & \multicolumn{3}{|c|}{ Storage in acre-feet } & \multirow[b]{2}{*}{$\begin{array}{c}\text { Storage } \\
\text { rat10 }\end{array}$} & \multirow[b]{2}{*}{ Use } & \multirow[b]{2}{*}{$\begin{array}{c}\text { Date } \\
\text { com- } \\
\text { pleted }\end{array}$} & \multirow[b]{2}{*}{$\begin{array}{l}\text { Owner } \\
\text { or } \\
\text { operator }\end{array}$} & \multirow{2}{*}{$\begin{array}{l}\text { Storage } \\
\text { recordd } \\
\text { pub- } \\
\text { lished }\end{array}$} \\
\hline & $\begin{array}{l}\text { Basin } \\
\text { 1ndex and } \\
\text { station no }\end{array}$ & $\begin{array}{l}\text { Lat1tude } \\
\text { and } \\
\text { long1tude }\end{array}$ & & & Dead & Total & Usable & & & & & \\
\hline PENNSYLVANIA--Cont1 nued & & & & & & & & & & & & \\
\hline $\begin{array}{l}\text { Pine Grove } \\
\text { Octoraro Creek }\end{array}$ & $01-578450$ & $\begin{array}{ll}39 & 48 \\
76 & 03\end{array}$ & 1396 & 669 & 0 & 8,590 & 8,590 & - & M & 1949 & $\begin{array}{l}\text { Chester Municipal } \\
\text { Authority }\end{array}$ & - \\
\hline $\begin{array}{l}\text { Piney } \\
\text { Clarion River }\end{array}$ & $\begin{array}{r}3 \mathrm{AF} \\
03-030400\end{array}$ & $\begin{array}{ll}41 & 11 \\
79 & 26\end{array}$ & 951 & 690 & 15,900 & 28,900 & 13,000 & $<0 \quad 05$ & $P$ & 1924 & $\begin{array}{l}\text { Pennsylvania Elec- } \\
\text { tric Co }\end{array}$ & $\mathrm{PA}$ \\
\hline $\begin{array}{l}\text { Prompton } \\
\text { West Branch Lackawaxen River }\end{array}$ & $01-428900$ & $\begin{array}{ll}41 & 35 \\
75 & 20\end{array}$ & 596 & - & 3,420 & 51,700 & 48,300 & 6 & $F$ & 1960 & Corps of Engineers & $\mathrm{PA}$ \\
\hline $\begin{array}{l}\text { Pymatuning } \\
\text { Shenango River }\end{array}$ & $\begin{array}{r}300 \\
03-100500\end{array}$ & $\begin{array}{ll}41 & 30 \\
80 & 28\end{array}$ & 158 & 16,722 & 10,155 & 198,200 & b159,900 & 117 & FMR & 1933 & $\begin{array}{l}\text { Commonwealth of } \\
\text { Pennsylvania }\end{array}$ & $\mathrm{PA}$ \\
\hline $\begin{array}{l}\text { Quemahon1ng } \\
\text { Quemahoning Creek }\end{array}$ & $\begin{array}{r}3 \mathrm{AK} \\
03-0394 \quad 50\end{array}$ & $\begin{array}{ll}40 & 11 \\
78 & 57\end{array}$ & 92 & 1,100 & 0 & 32,766 & 32,766 & 3 & MW & 1913 & $\begin{array}{l}\text { Manufacturers } \\
\text { Water Co }\end{array}$ & - \\
\hline $\begin{array}{l}\text { Raystown } \\
\text { Raystown Branch Juniata R1ver }\end{array}$ & $\begin{array}{r}1 \mathrm{PC} \\
01-562900\end{array}$ & $\begin{array}{ll}40 & 25 \\
78 & 01\end{array}$ & 949 & 500 & of & 9,000 & 9,000 & $<05$ & $P$ & 1911 & $\begin{array}{l}\text { Pennsylvania Elec- } \\
\text { tric Co }\end{array}$ & - \\
\hline $\begin{array}{l}\text { Safe Harbor } \\
\text { Susquehanna River }\end{array}$ & $01-5760^{1 P G}$ & $\begin{array}{ll}39 & 56 \\
76 & 23\end{array}$ & 26,117 & 7,328 & 75,129 & 144,000 & 68,900 & $<05$ & $P$ & 1931 & $\begin{array}{l}\text { Safe Harbor Water } \\
\text { Power Co }\end{array}$ & - \\
\hline $\begin{array}{l}\text { Shawnee } \\
\text { Shawnee Branch of Raystown Branch }\end{array}$ & $01-5597^{1 P C} 60$ & $\begin{array}{ll}40 & 02 \\
78 & 37\end{array}$ & 375 & 1,070 & 0 & 16,750 & 16,750 & 5 & $\mathrm{FR}$ & 1950 & $\begin{array}{l}\text { Commonwealth of } \\
\text { Pennsylvania }\end{array}$ & - \\
\hline $\begin{array}{l}\text { Still Creek } \\
\text { Still Creek }\end{array}$ & $01-4692^{1 \mathrm{LJ}}$ 00 & $\begin{array}{ll}40 & 51 \\
75 & 59\end{array}$ & 85 & - & 0 & 8,290 & 8,290 & 6 & M & 1933 & $\begin{array}{l}\text { Panther valley } \\
\text { Water Co }\end{array}$ & $\mathrm{PA}$ \\
\hline $\begin{array}{l}\text { Stillwater } \\
\text { Lackawanna River }\end{array}$ & $01-534180$ & $\begin{array}{ll}41 & 42 \\
75 & 29\end{array}$ & 371 & - & 200 & 12,000 & 11,800 & - & $\mathrm{FM}$ & 1959 & Corps of Engineers & PA \\
\hline $\begin{array}{l}\text { Thomas W Koon (Evitts Creek) } \\
\text { Evitts Creek }\end{array}$ & $01-6037^{1 S B}$ & $\begin{array}{ll}39 & 46 \\
78 & 40\end{array}$ & $\begin{array}{ll}44 & 8\end{array}$ & 305 & 46 & 7,090 & 7,040 & 2 & M & 1932 & $\begin{array}{l}\text { City of Cumberland } \\
\text { and Evitts Creek } \\
\text { Water Co }\end{array}$ & - \\
\hline $\begin{array}{l}\text { T1onesta Creek } \\
\text { T1onesta Creek }\end{array}$ & 03-0195 3 AD & $\begin{array}{ll}41 & 28 \\
79 & 26\end{array}$ & 478 & 2,770 & 0 & 133,400 & 133,400 & 2 & $\mathrm{FR}$ & 1941 & Corps of Engineers & $\mathrm{PA}, \mathrm{PF}$ \\
\hline $\begin{array}{l}\text { Watres } \\
\text { Spring Brook }\end{array}$ & 01-5355 1 50 & $\begin{array}{ll}41 & 17 \\
75 & 37\end{array}$ & $\begin{array}{ll}15 & 4\end{array}$ & 170 & 0 & 5,963 & 5,963 & 25 & M & 1925 & $\begin{array}{l}\text { Pennsylvania Gas } \\
\text { and Water Co }\end{array}$ & - \\
\hline $\begin{array}{l}\text { W1ld Creek } \\
\text { W1ld Creek }\end{array}$ & $\begin{array}{r}\text { ILH } \\
01-449700\end{array}$ & $\begin{array}{ll}40 & 54 \\
75 & 34\end{array}$ & 222 & 300 & 500 & 12,500 & 12,000 & 35 & M & 1941 & $\begin{array}{l}\text { Bethlehem Munic1pal } \\
\text { Water Authority }\end{array}$ & PA \\
\hline $\begin{array}{l}\text { Yough1ogheny } \\
\text { Youghiogheny R1ver }\end{array}$ & $\begin{array}{r}3 \mathrm{BJ} \\
01-077000\end{array}$ & $\begin{array}{ll}39 & 48 \\
79 & 22\end{array}$ & 434 & 3,566 & 0 & 254,000 & 254,000 & 4 & FRWY & 1943 & Corps of Englneers & $P A, P F$ \\
\hline
\end{tabular}




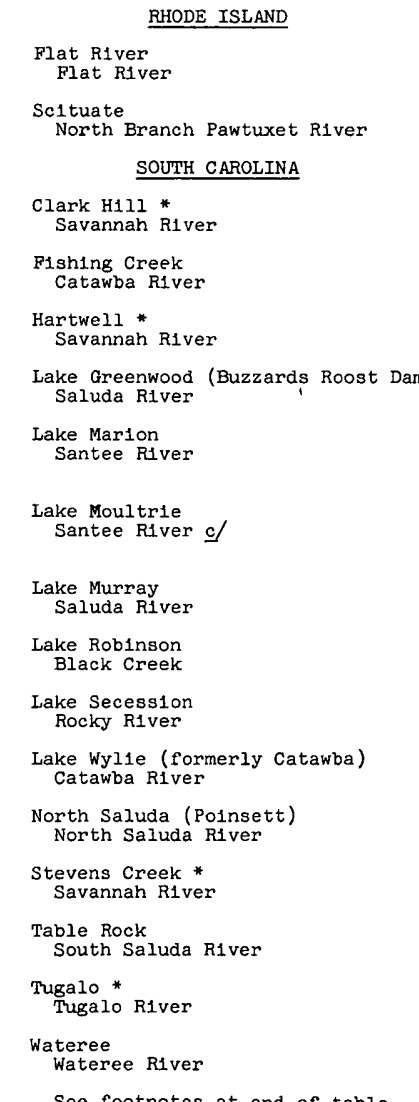

\begin{tabular}{|c|}
\hline $01-11599_{60}^{1 F F}$ \\
\hline $\begin{array}{r}1 \mathrm{FF} \\
01-11540^{5}\end{array}$ \\
\hline $02-1945^{2 J H}$ \\
\hline $02-1473^{2 G C} 90$ \\
\hline $02-1872^{2 J C}$ \\
\hline $02-1665$ 2GL \\
\hline $02-1710^{2 \mathrm{HB}}$ \\
\hline $02-1720$ $2 \mathrm{2HC}$ \\
\hline $\begin{array}{r}2 \mathrm{GL} \\
02-1685^{\circ} 00\end{array}$ \\
\hline $02-1309^{2 F G}$ O9 \\
\hline $02-1879^{2 J C} 50$ \\
\hline $02-1459^{2 G C} 33$ \\
\hline $02-162325$ \\
\hline $02-196450$ \\
\hline $02-1620^{2 \mathrm{GK}}$ \\
\hline $02-181600$ \\
\hline $02-1479^{2 G D} 00$ \\
\hline
\end{tabular}

\begin{tabular}{|c|c|}
\hline $\begin{array}{ll}41 & 41 \\
71 & 35\end{array}$ & - \\
\hline $\begin{array}{ll}41 & 45 \\
71 & 35\end{array}$ & 928 \\
\hline $\begin{array}{ll}33 & 40 \\
82 & 12\end{array}$ & 6,150 \\
\hline $\begin{array}{ll}34 & 36 \\
80 & 53\end{array}$ & 3,810 \\
\hline $\begin{array}{ll}34 & 21 \\
82 & 50\end{array}$ & 2,088 \\
\hline $\begin{array}{ll}34 & 10 \\
81 & 54\end{array}$ & 1,150 \\
\hline $\begin{array}{ll}33 & 27 \\
80 & 10\end{array}$ & 14,700 \\
\hline $\begin{array}{ll}33 & 15 \\
80 & 00\end{array}$ & - \\
\hline $\begin{array}{ll}34 & 03 \\
81 & 13\end{array}$ & 2,420 \\
\hline $\begin{array}{ll}34 & 24 \\
80 & 09\end{array}$ & 173 \\
\hline $\begin{array}{ll}34 & 15 \\
82 & 37\end{array}$ & 199 \\
\hline $\begin{array}{ll}35 & 01 \\
81 & 00\end{array}$ & 3,020 \\
\hline $\begin{array}{ll}35 & 08 \\
82 & 24\end{array}$ & 28 \\
\hline $\begin{array}{ll}33 & 34 \\
82 & 03\end{array}$ & 7,180 \\
\hline $\begin{array}{ll}35 & 04 \\
82 & 40\end{array}$ & 145 \\
\hline $\begin{array}{ll}34 & 41 \\
83 & 21\end{array} \mid$ & 465 \\
\hline $\begin{array}{ll}34 & 20 \\
80 & 42\end{array}$ & 4,750 \\
\hline
\end{tabular}

\begin{tabular}{r|c|}
974 & - \\
3,600 & - \\
78,500 & $1,170,000$ \\
3,370 & 22,268 \\
61,350 & $1,134,100$ \\
11,800 & 80,300 \\
115,000 & 350,100 \\
65,000 & 450,000 \\
50,800 & 500,000 \\
2,145 & - \\
$a 3,000$ & $a 50,000$ \\
12,455 & 129,890 \\
1,080 & 0 \\
4,300 & 5,000 \\
500 & 12,280 \\
53,710 & 31,500 \\
134,848 \\
\end{tabular}
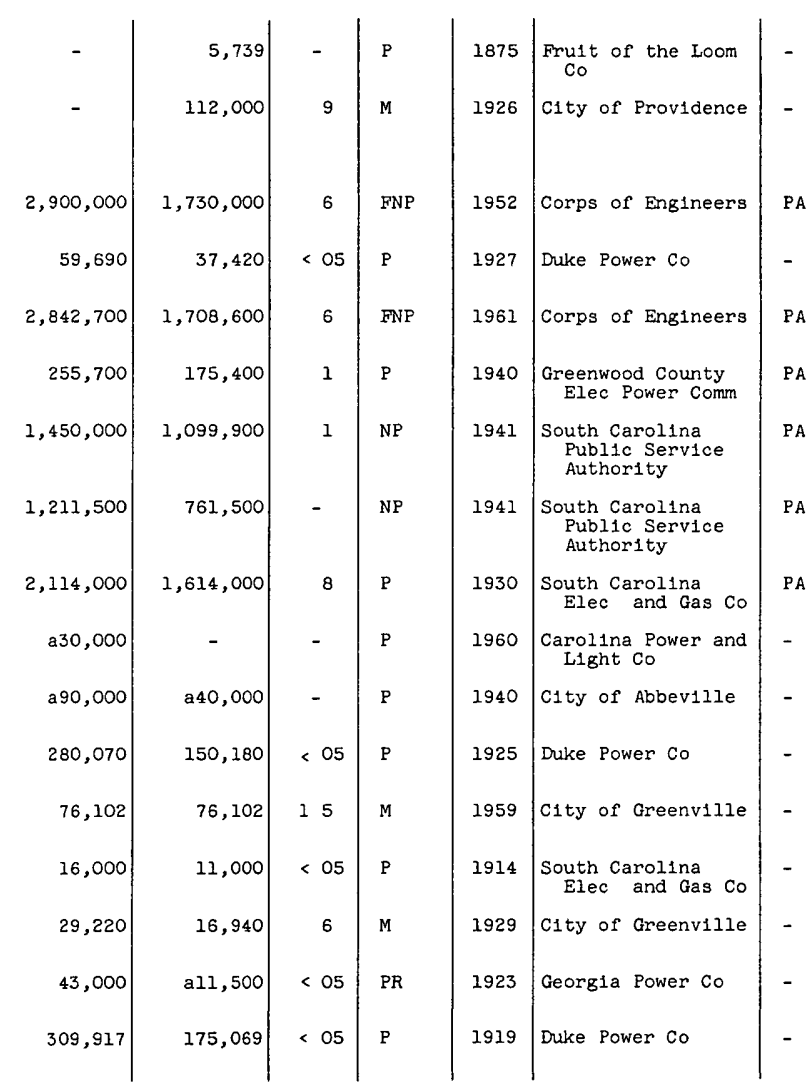

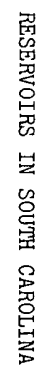


Table 1 --Reservo1rs in the Un1ted States completed or under construction as of January 1, 1963, having a usable capac1ty of 5, o0o acre-feet or more--Continued

\begin{tabular}{|c|c|c|c|c|c|c|c|c|c|c|c|c|}
\hline \multirow[b]{2}{*}{$\begin{array}{l}\text { Name of reservolr } \\
\text { and } \\
\text { stream }\end{array}$} & \multicolumn{2}{|c|}{ Location } & \multirow{2}{*}{$\begin{array}{l}\text { Drainage } \\
\text { area } \\
\text { (square } \\
\text { m1les) }\end{array}$} & \multirow[b]{2}{*}{$\begin{array}{c}\text { Surface } \\
\text { area } \\
\text { (acres) }\end{array}$} & \multicolumn{3}{|c|}{ Storage in acre-feet } & \multirow[b]{2}{*}{$\begin{array}{r}\text { Storage } \\
\text { rat1o }\end{array}$} & \multirow[b]{2}{*}{ Use } & \multirow[b]{2}{*}{$\begin{array}{c}\text { Date } \\
\text { com- } \\
\text { pleted }\end{array}$} & \multirow[b]{2}{*}{$\begin{array}{l}\text { Owner } \\
\text { or } \\
\text { operator }\end{array}$} & \multirow{2}{*}{$\begin{array}{c}\text { Storage } \\
\text { records } \\
\text { pub- } \\
\text { lished }\end{array}$} \\
\hline & \begin{tabular}{|c|} 
Basin \\
1ndex and \\
station no
\end{tabular} & $\begin{array}{l}\text { Lat1 tude } \\
\text { and } \\
\text { long1tude }\end{array}$ & & & Dead & Total & Usable & & & & & \\
\hline \multicolumn{13}{|l|}{ SOUTH DAKOTA } \\
\hline $\begin{array}{l}\text { Angostura } \\
\text { Cheyenne River }\end{array}$ & $\begin{array}{r}6 \mathrm{KH} \\
06-4010^{0}\end{array}$ & $\begin{array}{rr}43 & 21 \\
103 & 26\end{array}$ & 9,100 & 4,830 & 23,830 & 160,100 & 136,300 & 07 & FIPR & 1949 & $\begin{array}{l}\text { Bureau of } \\
\text { Reclamation }\end{array}$ & PA \\
\hline $\begin{array}{l}\text { Belle Fourche (formerly Orman) } \\
\text { Belle Fourche River } c \text { f }\end{array}$ & $\begin{array}{r}6 \mathrm{KD} \\
06-4350 \quad 00\end{array}$ & $\begin{array}{rr}44 & 44 \\
103 & 40\end{array}$ & - & 8,040 & 6,800 & 192,000 & 185,200 & - & IR & 1911 & $\begin{array}{l}\text { Bureau of } \\
\text { Reclamation }\end{array}$ & PA \\
\hline $\begin{array}{l}\text { Big Bend } \\
\text { M1ssour1 River }\end{array}$ & $06-442700$ & $\begin{array}{ll}44 & 03 \\
99 & 27\end{array}$ & 249,330 & 55,800 & 384,000 & $1,900,000$ & $1,516,000$ & 1 & FNPR & $\begin{array}{l}\text { Under } \\
\text { const }\end{array}$ & Corps of Engineers & - \\
\hline $\begin{array}{l}\text { Deerfield } \\
\text { Castle Creek }\end{array}$ & $06-4095 \quad 600$ & $\begin{array}{rr}44 & 02 \\
103 & 47\end{array}$ & 95 & 414 & 565 & 15,718 & 15,153 & 24 & IMR & 1945 & $\begin{array}{l}\text { Bureau of } \\
\text { Reclamation }\end{array}$ & PA \\
\hline $\begin{array}{l}\text { Fort Randali } \\
\text { Missouri River }\end{array}$ & $06-452500$ & $\begin{array}{ll}43 & 04 \\
98 & 33\end{array}$ & 263,500 & 102,600 & 0 & $6,093,000$ & $6,093,000$ & 3 & FINPR & 1956 & Corps of Engineers & PA \\
\hline $\begin{array}{l}\text { Lake Sheridan } \\
\text { Spring Creek }\end{array}$ & $06-406980$ & $\begin{array}{rr}43 & 58 \\
103 & 27\end{array}$ & - & 378 & - & 12,660 & - & - & $\mathrm{R}$ & 1941 & Forest Service & - \\
\hline $\begin{array}{l}\text { Lake Traverse (Wh1te Rock Dam) * } \\
\text { Bo1se de S1oux R1ver }\end{array}$ & 05-0499 $50 \mathrm{~A}$ & $\begin{array}{ll}45 & 52 \\
96 & 34\end{array}$ & 1,160 & 22,975 & b112,500 & 249,500 & 137,000 & 267 & F & 1941 & Corps of Engineers & PA \\
\hline $\begin{array}{l}\text { Lew1s and Clark Lake (Gavins Polnt } \\
\text { Dam) * } \\
\text { Missouri River }\end{array}$ & $\begin{array}{r}6 \mathrm{LN} \\
06-4670 \quad 00\end{array}$ & $\begin{array}{ll}42 & 51 \\
97 & 29\end{array}$ & 279,500 & 17,000 & 18,000 & 541,000 & 523,000 & $<05$ & FINPR & 1956 & Corps of Englneers & PA \\
\hline $\begin{array}{l}\text { Mitchell } \\
\text { Firesteel Creek }\end{array}$ & $\begin{array}{r}6 L R \\
06-477600\end{array}$ & $\begin{array}{ll}43 & 44 \\
98 & 01\end{array}$ & 584 & 790 & 2300 & 10,560 & 8,260 & 3 & M & 1928 & C1ty of M1tchell & - \\
\hline $\begin{array}{l}\text { Oahe } \\
\text { Missour1 R1ver }\end{array}$ & $06-4399^{6 L A}$ & $\begin{array}{rr}44 & 27 \\
100 & 25\end{array}$ & 243,500 & 376,000 & 2,000 & $23,630,000$ & $23,628,000$ & 14 & FINPR & $\begin{array}{l}\text { Under } \\
\text { const }\end{array}$ & Corps of Engineers & PA \\
\hline $\begin{array}{l}\text { Pactola } \\
\quad \text { Rapid Creek }\end{array}$ & $06-4110 \quad 00$ & \begin{tabular}{rr|}
44 & 04 \\
103 & 29
\end{tabular} & 319 & 1,230 & 140 & 99,100 & 98,960 & 33 & FIMR & 1956 & $\begin{array}{l}\text { Bureau of } \\
\text { Reclamation }\end{array}$ & PA \\
\hline $\begin{array}{l}\text { Shadehill } \\
\text { Grand RIver }\end{array}$ & $06-357000$ & $\begin{array}{rr}45 & 46 \\
102 & 12\end{array}$ & 3,120 & 4,800 & 58,200 & 140,000 & 81,400 & 6 & FI & 1951 & $\begin{array}{l}\text { Bureau of } \\
\text { Reclamation }\end{array}$ & PA \\
\hline \multicolumn{13}{|l|}{ TENNESSEE } \\
\hline $\begin{array}{l}\text { Boone Lake } \\
\text { South Fork Holston River }\end{array}$ & $\begin{array}{r}3 T B \\
03-4868 \quad 00\end{array}$ & $\begin{array}{ll}36 & 26 \\
82 & 26\end{array}$ & 1,840 & 4,520 & 46,700 & 196,700 & 150,000 & - & FNPR & 1952 & $\begin{array}{l}\text { Tennessee valley } \\
\text { Authority }\end{array}$ & $\mathrm{PA}, \mathrm{PE}$ \\
\hline $\begin{array}{l}\text { Center Hill } \\
\text { Caney Fork }\end{array}$ & $03-4240 \quad 00$ & $\begin{array}{ll}36 & 06 \\
85 & 50\end{array}$ & 2,195 & 23,060 & 838,000 & $2,092,000$ & b1, 254,000 & - & FPR & 1948 & Corps of Eng1neers & $P A, P E$ \\
\hline $\begin{array}{l}\text { Cheatham Lake } \\
\text { Cumberland River }\end{array}$ & $\left|\begin{array}{r}3 S C \\
03-434900\end{array}\right|$ & $\begin{array}{ll}36 & 19 \\
87 & 13\end{array}$ & 14,160 & 7,450 & 84,200 & 104,000 & 19,800 & - & NPR & 1954 & Corps of Engineers & PE \\
\hline
\end{tabular}


Cherokee Lake
Holston River

Chickamauga Lake

Chilhowee Lake

L1ttle Tennessee River
Lake

Dale Hollow

Douglas Lake

French Broad River

Elk River (Woods Dam)

E1k R1ver

Fort Loudoun Lake

Great Falls Lake (Rock Island)

Caney Fork River

Hales Bar Lake

Melton Hill

clinch River

Norris Lake

Ocoee No 1 (formerly Parksville Ocoee RIver

old Hickory Lake

Pickwick Lake

Tennessee River

South Holston Lake
South Fork Holston River

Watauga Lake

Watauga R1ver

Watts Bar Lake
Tennessee River

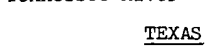

Abbott TP3 (Lake Mc

Guadalupe River

See footnotes at end of table

\begin{tabular}{|c|c|c|}
\hline $\begin{array}{r}3 \mathrm{TE} \\
03-493500\end{array}$ & $\begin{array}{ll}36 & 10 \\
83 & 30\end{array}$ & 3,428 \\
\hline $\begin{array}{r}\text { 3VA } \\
03-5665 \text { OO }\end{array}$ & $\begin{array}{ll}35 & 06 \\
85 & 14\end{array}$ & 20,790 \\
\hline $\begin{array}{r}3 T R \\
03-518200\end{array}$ & $\begin{array}{ll}35 & 33 \\
84 & 03\end{array} \mid$ & 1,976 \\
\hline $\begin{array}{r}3 \mathrm{RH} \\
03-416500\end{array}$ & $\begin{array}{ll}36 & 32 \\
85 & 27\end{array}$ & 935 \\
\hline $03-4685$ TTL & $\begin{array}{ll}35 & 58 \\
83 & 32\end{array}$ & 4,541 \\
\hline $\begin{array}{r}3 \mathrm{VH} \\
03-5790 \text { 00 }\end{array}$ & $\begin{array}{ll}35 & 18 \\
86 & 06\end{array}$ & 263 \\
\hline $\begin{array}{r}3 U G \\
03-4995 \text { 00 }\end{array}$ & $\begin{array}{ll}35 & 48 \\
84 & 15\end{array}$ & 9,550 \\
\hline $\begin{array}{r}3 R K \\
03-4220 \text { 00 }\end{array}$ & $\begin{array}{ll}35 & 48 \\
85 & 38\end{array}$ & 1,677 \\
\hline $\begin{array}{r}3 \mathrm{VB} \\
03-569500\end{array}$ & $\begin{array}{ll}35 & 03 \\
85 & 32\end{array}$ & 21,790 \\
\hline $\begin{array}{r}\text { 3UG } \\
03-5359 \text { 00 }\end{array}$ & $\begin{array}{ll}35 & 53 \\
84 & 18\end{array}$ & 3,343 \\
\hline $\begin{array}{r}\text { 3UG } \\
03-5325 \text { 00 }\end{array}$ & $\begin{array}{ll}36 & 13 \\
84 & 05\end{array}$ & 2,912 \\
\hline $\begin{array}{r}3 \mathrm{UKK} \\
03-5640 \text { OO }\end{array}$ & $\begin{array}{ll}35 & 06 \\
84 & 39\end{array}$ & 595 \\
\hline $\begin{array}{r}3 S A \\
03-426300\end{array}$ & $\begin{array}{ll}36 & 18 \\
86 & 39\end{array}$ & 11,674 \\
\hline $\begin{array}{r}3 W A \\
03-5930 \text { OD }\end{array}$ & $\begin{array}{ll}35 & 04 \\
88 & 15\end{array}$ & 32,820 \\
\hline $\begin{array}{r}3 \mathrm{~TB} \\
03-4760 \quad 00\end{array}$ & $\begin{array}{ll}36 & 31 \\
82 & 05\end{array}$ & 703 \\
\hline $\begin{array}{r}3 \mathrm{TC} \\
03-4835 \text { OO }\end{array}$ & $\begin{array}{ll}36 & 19 \\
82 & 07\end{array}$ & 468 \\
\hline $\begin{array}{r}\text { 3UA } \\
03-5430 \text { OO }\end{array}$ & $\begin{array}{ll}35 & 37 \\
84 & 47\end{array}$ & 17,310 \\
\hline $\begin{array}{r}8 \mathrm{KE} \\
08-1697 \text { 00 }\end{array}$ & $\begin{array}{ll}29 & 36 \\
98 & 02\end{array}$ & 1,684 \\
\hline
\end{tabular}

\begin{tabular}{|c|c|c|c|c|c|}
\hline 31,100 & b92,300 & $1,565,400$ & $b_{1}, 473,100$ & - & FNPR \\
\hline 39,400 & b3 35,900 & $70 \varepsilon, 300$ & b329,400 & - & FNPR \\
\hline 1,690 & 42,450 & 49,250 & 6,800 & - & FNP \\
\hline 30,990 & b857,000 & $1,706,000$ & b849,000 & 74 & FPR \\
\hline 31,600 & b 94,400 & $1,514,100$ & $1,419,700$ & 30 & FNPR \\
\hline 4,275 & 68,570 & 79,870 & b19,450 & 06 & FRW \\
\hline 15,500 & b277,200 & 386,500 & b109,300 & - & FNPR \\
\hline 2,270 & b5,100 & 54,500 & $\mathrm{~b} 49,400$ & $<05$ & PR \\
\hline 6,680 & b 135,300 & 147,700 & bl2,400 & - & FNPR \\
\hline - & 92,500 & 118,500 & 26,000 & - & NP \\
\hline 40,200 & b286,000 & $2,567,000$ & b2,281,000 & 73 & FNPR \\
\hline 1,900 & b58,200 & d91,300 & $\mathrm{d} 33,100$ & - & PR \\
\hline 27,450 & 357,000 & 545,000 & 188,000 & - & NPR \\
\hline 46,800 & 673,000 & $1,091,400$ & 418,400 & - & FNPR \\
\hline 8,750 & bl18,800 & 744,000 & b625,200 & 92 & FNPR \\
\hline 7,200 & 51,600 & 678,800 & 627,200 & 133 & FNPR \\
\hline 43,100 & b 754,400 & $1,132,000$ & 377,600 & - & FNPR \\
\hline 39 & 이 & 5,000 & 5,000 & $<05$ & $P$ \\
\hline
\end{tabular}

$P A, P E$

$P A, P E$

$P A, P E$

PA, PE

$P A, P E$

PA

PA, $P E$

PA, PE

$P A, P E$

$\mathrm{PA}, \mathrm{PE}$

$\mathrm{PA}, \mathrm{PE}$

A, PE

PA

PA, PE

PE 
Table 1 --Reservo1rs in the United States completed or under construction as of January 1, 1963, having a usable capac1ty of 5,000 acre-feet or more--Continued

\begin{tabular}{|c|c|c|c|c|c|c|c|c|c|c|c|c|}
\hline \multirow[b]{2}{*}{$\begin{array}{l}\text { Name of reservo1r } \\
\text { and } \\
\text { stream }\end{array}$} & \multicolumn{2}{|c|}{ Location } & \multirow{2}{*}{$\begin{array}{l}\text { Drainage } \\
\text { area } \\
\text { (square } \\
\text { miles) }\end{array}$} & \multirow[b]{2}{*}{$\begin{array}{c}\text { Surface } \\
\text { area } \\
\text { (acres) }\end{array}$} & \multicolumn{3}{|c|}{ Storage in acre-feet } & \multirow[b]{2}{*}{$\begin{array}{c}\text { Storage } \\
\text { rat10 }\end{array}$} & \multirow[b]{2}{*}{ Use } & \multirow[b]{2}{*}{\begin{tabular}{|c|} 
Date \\
com- \\
pleted
\end{tabular}} & \multirow[b]{2}{*}{$\begin{array}{l}\text { Owner } \\
\text { or } \\
\text { operator }\end{array}$} & \multirow{2}{*}{$\begin{array}{l}\text { Storage } \\
\text { records } \\
\text { pub- } \\
\text { l1shed }\end{array}$} \\
\hline & \begin{tabular}{|c|} 
Basin \\
1ndex and \\
station no
\end{tabular} & $\begin{array}{l}\text { Lat1tude } \\
\text { and } \\
\text { long1tude }\end{array}$ & & & Dead & Total & Usable & & & & & \\
\hline TEXAS--Cont1nued & & & & & & & & & & & & \\
\hline $\begin{array}{l}\text { Addicks } \\
\text { South Mayde Creek }\end{array}$ & O8-0730 00 & $\begin{array}{ll}29 & 47 \\
95 & 37\end{array}$ & 133 & 16,780 & - & 204,460 & 188,030 & 26 & $\mathrm{~F}$ & 1948 & Corps of Engineers & PA \\
\hline $\begin{array}{l}\text { Alcoa Lake } \\
\text { Sandy Creek } c \text { / }\end{array}$ & $\begin{array}{r}8 M E \\
08-109600\end{array}$ & $\begin{array}{ll}30 & 35 \\
97 & 03\end{array}$ & - & 703 & of & 10,500 & 10,500 & - & $\mathrm{RW}$ & 1953 & $\underset{\substack{\text { America } \\
\text { America }}}{\text { of }}$ & - \\
\hline $\begin{array}{l}\text { Anahuac Lake } \\
\text { Turtle Bay } \mathrm{c}\end{array}$ & $08-067350$ & $\begin{array}{ll}29 & 46 \\
94 & 41\end{array}$ & - & 5,300 & 0 & 35,300 & 35,300 & - & I & 1941 & $\begin{array}{l}\text { Chambers-Liberty } \\
\text { Co's Navigation } \\
\text { District }\end{array}$ & - \\
\hline $\begin{array}{l}\text { Barker } \\
\text { Buffalo Bayou }\end{array}$ & 08-0725 00 & $\begin{array}{ll}29 & 46 \\
95 & 39\end{array}$ & 134 & 17,225 & - & 204,800 & 127,900 & 14 & $\mathrm{~F}$ & 1946 & Corps of Engineers & PA \\
\hline $\begin{array}{l}\text { Baylor Creek } \\
\text { Baylor Creek }\end{array}$ & $07-2995^{7 \mathrm{KA}}$ & $\begin{array}{rr}34 & 28 \\
100 & 22\end{array}$ & 40 & 610 & 0 & 9,220 & 9,220 & 4 & M & 1950 & C1ty of Childress & - \\
\hline $\begin{array}{l}\text { Belton } \\
\text { Leon River }\end{array}$ & $08-102000$ & $\begin{array}{ll}31 & 07 \\
97 & 28\end{array}$ & 3,499 & 23,620 & 280 & $1,097,600$ & $1,097,300$ & 22 & FIMW & 1954 & Corps of Engineers & $\mathrm{PA}$ \\
\hline $\begin{array}{l}\text { Benbrook } \\
\text { Clear Fork Trinity River }\end{array}$ & $08-046500$ & $\begin{array}{ll}32 & 39 \\
97 & 27\end{array}$ & 429 & 5,820 & 10 & 164,800 & 164,800 & 37 & FN & 1950 & Corps of Engineers & PA \\
\hline $\begin{array}{l}\text { Bivins Lake } \\
\text { Palo Duro Creek }\end{array}$ & $07-2956$ \% 00 & $\begin{array}{rr}35 & 02 \\
102 & 02\end{array}$ & 982 & 379 & 0 & 5,120 & 5,120 & 2 & M & 1927 & City of Amarillo & PA \\
\hline $\begin{array}{l}\text { Brazoria } \\
\text { Brazos River } \subseteq\end{array}$ & $08-117300$ & $\begin{array}{ll}29 & 04 \\
95 & 33\end{array}$ & - & 1,865 & 650 & 21,970 & 21,320 & - & w & 1954 & Dow Chemical Co & - \\
\hline $\begin{array}{l}\text { Bridgeport } \\
\text { West Fork Trinity River }\end{array}$ & $\begin{array}{r}8 \mathrm{NB} \\
08-0430 \quad 00\end{array}$ & $\begin{array}{ll}33 & 13 \\
97 & 50\end{array}$ & 1,111 & 10,400 & 0 & 270,900 & 270,000 & 17 & IMW & 1931 & $\begin{array}{l}\text { Tarrant County hater } \\
\text { Control and } \\
\text { Improv Dist Na.1 }\end{array}$ & $P A$ \\
\hline $\begin{array}{l}\text { Brownwood } \\
\text { Pecan Bayou }\end{array}$ & $08-1430 \quad 000$ & $\begin{array}{ll}31 & 50 \\
99 & 00\end{array}$ & 1,535 & 7,300 & 0 & 143,400 & 143,400 & 9 & IMW & 1933 & $\begin{array}{l}\text { Brown County Water } \\
\text { Improvement } \\
\text { District No } 1\end{array}$ & $P A$ \\
\hline $\begin{array}{l}\text { Brushy Creek } \\
\text { Brushy Creek }\end{array}$ & $07-332100$ & $\begin{array}{ll}33 & 38 \\
96 & 22\end{array}$ & 8 & 1,180 & 0 & 16,800 & 16,800 & 5 & w & 1961 & $\begin{array}{l}\text { Texas Power and } \\
\text { L1ght Co }\end{array}$ & - \\
\hline $\begin{array}{l}\text { Buchanan } \\
\text { Colorado RIver }\end{array}$ & $08-148000$ & $\begin{array}{ll}30 & 45 \\
98 & 25\end{array}$ & 31,250 & 23,200 & 36,800 & 992,200 & 955,200 & 7 & IMPW & 1937 & $\begin{array}{l}\text { Lower Colorado } \\
\text { River Authority }\end{array}$ & PA \\
\hline $\begin{array}{l}\text { Buffalo Lake } \\
\text { Tlerra Blanca Creek }\end{array}$ & $\begin{array}{r}7 K A \\
07-2960 \\
00\end{array}$ & $\begin{array}{rr}34 & 55 \\
102 & 06\end{array}$ & 2,075 & al,, 900 & 0 & 18,150 & 18,150 & 20 & $R$ & 1938 & $\begin{array}{l}\text { F1sh and Wildilfe } \\
\text { Service }\end{array}$ & PA \\
\hline $\begin{array}{l}\text { Caddo Lake } \\
\text { Cypress Creek }\end{array}$ & $\left|\begin{array}{r}7 \mathrm{MA} \\
07-3463 \quad 10\end{array}\right|$ & $\begin{array}{ll}32 & 40 \\
94 & 10\end{array}$ & 2,639 & 32,700 & 0 & 175,000 & 175,000 & 1 & $\mathrm{MN}$ & 1914 & Corps of Engineers & PL \\
\hline
\end{tabular}




Camp Creek Lake
Camp Creek
Casa Blanca Lake
Chacon Creek
Champlon Creek
Champion Creek
Devils Lake
Devils River
Eagle Lake
Colorado River c/
Eagle Mountain
West Fork Trin1ty River
Eagle Nest and Manor Lake
Varner Creek c/
Ellison Creek
El11son Creek
Farmers Creek (Nacona)
Farmers Creek
Flat Creek
Flat Creek
Fort Phantom Hill
B1g Elm Creek
Garza-L1ttle Elm
Elm Fork Trinity River
Granite Shoals Lake
Colorado R1ver
Grapevine
Denton Creek
H-4 Development
Guadalupe River
H1ghlands
Goose Creek c/
Hords Creek
Hords Creek
Hubbard Creek
Hubbard Creek
See footnotes at end of table

\begin{tabular}{|c|c|c|c|}
\hline $\begin{array}{r}8 M E \\
08-1107 \quad 00\end{array}$ & $\begin{array}{ll}31 & 03 \\
96 & 18\end{array} \mid$ & 40 & 750 \\
\hline $\begin{array}{c}8 \mathrm{~J} \\
08-4591 \stackrel{00}{0}\end{array}$ & $\begin{array}{ll}27 & 32 \\
99 & 26\end{array}$ & 117 & 1,656 \\
\hline $\begin{array}{r}\text { 8LA } \\
08-1236 \text { }\end{array}$ & $\begin{array}{rr}32 & 17 \\
100 & 51\end{array}$ & 203 & 1,560 \\
\hline $08-4494 \quad 60$ & $\begin{array}{rr}29 & 34 \\
100 & 59\end{array}$ & 3,872 & 406 \\
\hline $08-1616$ OLF & $\begin{array}{ll}29 & 33 \\
96 & 20\end{array}$ & - & 1,200 \\
\hline $\begin{array}{r}8 \mathrm{NB} \\
08-045000\end{array}$ & $\begin{array}{ll}32 & 53 \\
97 & 28\end{array}$ & 1,970 & 8,500 \\
\hline $\begin{array}{r}8 \mathrm{ME} \\
08-1169 \stackrel{00}{0}\end{array}$ & $\begin{array}{ll}29 & 13 \\
95 & 38\end{array}$ & - & 4,305 \\
\hline $07-3455$ 7MA & $\begin{array}{ll}32 & 55 \\
94 & 44\end{array}$ & 37 & 1,516 \\
\hline $\begin{array}{r}7 \mathrm{KC} \\
07-3156 \quad 00\end{array}$ & $\begin{array}{ll}33 & 53 \\
97 & 39\end{array}$ & 94 & 1,470 \\
\hline $\begin{array}{r}80 \mathrm{~B} \\
08-0312^{9}\end{array}$ & $\begin{array}{ll}32 & 12 \\
95 & 43\end{array}$ & 21 & 1,520 \\
\hline $\begin{array}{r}8 \mathrm{MB} \\
08-083500\end{array}$ & $\begin{array}{ll}32 & 37 \\
99 & 40\end{array}$ & 478 & 4,246 \\
\hline $08-052800$ & $\begin{array}{ll}33 & 04 \\
96 & 58\end{array}$ & 1,660 & 38,900 \\
\hline $08-1482$ OD & $\begin{array}{ll}30 & 33 \\
98 & 20\end{array}$ & 36,290 & 6,400 \\
\hline $\begin{array}{r}8 \mathrm{BNB} \\
08-054500\end{array}$ & $\begin{array}{ll}32 & 58 \\
97 & 03\end{array}$ & 695 & 12,740 \\
\hline $\begin{array}{r}\text { 8KE } \\
08-169800\end{array}$ & $\begin{array}{ll}29 & 29 \\
97 & 37\end{array}$ & 2,038 & 800 \\
\hline $\begin{array}{r}8 \mathrm{NA} \\
08-072100\end{array}$ & $\begin{array}{ll}29 & 50 \\
95 & 02\end{array}$ & - & 1,407 \\
\hline $08-1410^{8 L C}$ & $\begin{array}{ll}31 & 50 \\
99 & 34\end{array}$ & 48 & 510 \\
\hline $\begin{array}{r}8 M B \\
08-086400\end{array}$ & $\begin{array}{ll}32 & 50 \\
98 & 58\end{array}$ & 1,107 & 15,250 \\
\hline
\end{tabular}
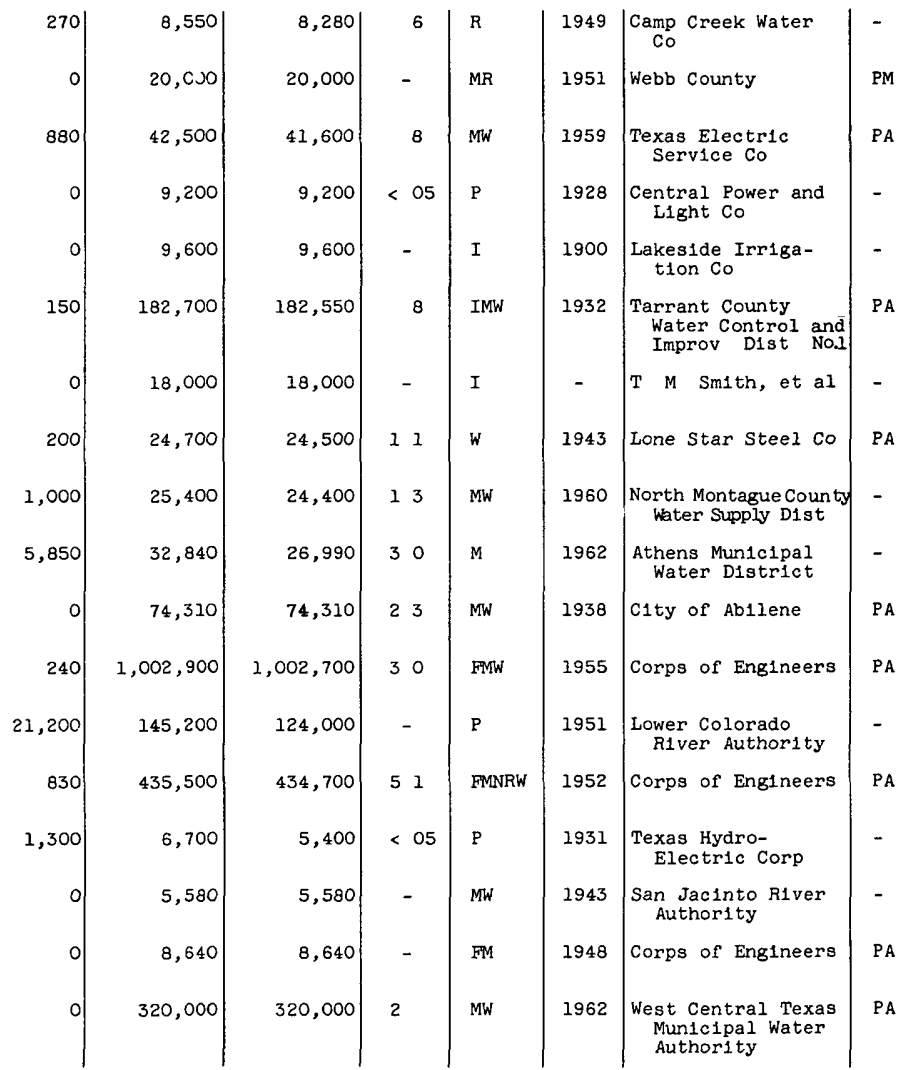
Table 1 --Reservo1rs in the United States completed or under construction as of January 1, 1963, hav1ng a usable capac1ty of 5,000 acre-feet or more--Continued

\begin{tabular}{|c|c|c|c|c|c|c|c|c|c|c|c|c|}
\hline \multirow[b]{2}{*}{$\begin{array}{l}\text { Name of reservo1r } \\
\text { and } \\
\text { stream }\end{array}$} & \multicolumn{2}{|c|}{ Location } & \multirow{2}{*}{$\begin{array}{l}\text { Drainage } \\
\text { area } \\
\text { (square } \\
\text { miles) }\end{array}$} & \multirow[b]{2}{*}{$\begin{array}{c}\text { Surface } \\
\text { area } \\
\text { (acres) }\end{array}$} & \multicolumn{3}{|c|}{ Storage in acre-feet } & \multirow[b]{2}{*}{$\mid \begin{array}{c}\text { Storage } \\
\text { rat10 }\end{array}$} & \multirow[b]{2}{*}{ Use } & \multirow[b]{2}{*}{$\begin{array}{c}\text { Date } \\
\text { com- } \\
\text { pleted }\end{array}$} & \multirow[b]{2}{*}{$\begin{array}{l}\text { Owner } \\
\text { or } \\
\text { operator }\end{array}$} & \multirow{2}{*}{$\begin{array}{l}\text { Storage } \\
\text { records } \\
\text { pub- } \\
\text { lished }\end{array}$} \\
\hline & \begin{tabular}{|c|} 
Bas1n \\
1ndex and \\
station no
\end{tabular} & $\begin{array}{l}\text { Lat1tude } \\
\text { and } \\
\text { long1tude }\end{array}$ & & & Dead & Total & Usable & & & & & \\
\hline TEXAS--Cont1nued & & & & & & & & & & & & \\
\hline $\begin{array}{l}\text { Inks } \\
\text { Colorado River }\end{array}$ & $08-148100$ & $\begin{array}{ll}30 & 44 \\
98 & 23\end{array}$ & 31,290 & 830 & o) & 17,000 & 17,000 & - & $\mathrm{P}$ & 1938 & $\begin{array}{l}\text { Lower Colorado } \\
\text { River Authority }\end{array}$ & - \\
\hline $\begin{array}{l}\text { International Falcon } \\
\text { R10 Grande }\end{array}$ & $\mid \begin{array}{c}8 \mathrm{~J} \\
08-461200\end{array}$ & $\begin{array}{ll}26 & 33 \\
99 & 10\end{array}$ & 164,482 & 78,340 & 2,820 & $3,280,700$ & $3,277,900$ & 094 & FIMPW & 1953 & $\begin{array}{l}\text { U S and Mexico In- } \\
\text { ternational Bound- } \\
\text { ary and Water Comm }\end{array}$ & PM \\
\hline $\begin{array}{l}\text { Johnson Creek } \\
\text { Johnson Creek }\end{array}$ & $\begin{array}{r}7 M A \\
07-3458 \quad 00\end{array}$ & $\begin{array}{ll}32 & 51 \\
94 & 33\end{array}$ & 11 & 650 & 0 & 10,100 & 10,100 & 14 & w & 1961 & $\begin{array}{l}\text { Southwestern Elec- } \\
\text { tric Power Co }\end{array}$ & - \\
\hline $\begin{array}{l}\text { Kirby Lake } \\
\text { Cedar Creek }\end{array}$ & 08-0834 50 & $\begin{array}{ll}32 & 23 \\
99 & 44\end{array}$ & 44 & 740 & 0 & 7,620 & 7,620 & 15 & IMR & 1928 & City of Abilene & - \\
\hline $\begin{array}{l}\text { Lake Abilene } \\
\text { Elm Creek }\end{array}$ & $08-083250$ & $\begin{array}{ll}32 & 14 \\
99 & 53\end{array}$ & 102 & 641 & 0 & 9,790 & 9,790 & 16 & M & 1921 & City of Abilene & - \\
\hline $\begin{array}{l}\text { Lake Amon G Carter } \\
\text { B1g Sandy Creek }\end{array}$ & $\begin{array}{r}8 \mathrm{8NB} \\
08-043700\end{array}$ & $\begin{array}{ll}33 & 28 \\
97 & 52\end{array}$ & 100 & 1,540 & 0 & 20,050 & 20,050 & 10 & MW & 1956 & C1ty of Bowie & - \\
\hline $\begin{array}{l}\text { Lake Arlington } \\
\text { Village Creek }\end{array}$ & 08-0492 80 & $\begin{array}{ll}32 & 43 \\
97 & 12\end{array}$ & 143 & 2,275 & 180 & 45,710 & 45,530 & 13 & MW & 1957 & C1ty of Arlington & PA \\
\hline $\begin{array}{l}\text { Lake Austin } \\
\text { Colorado River }\end{array}$ & $08-1549^{8 \mathrm{LF}}$ & $\begin{array}{ll}30 & 18 \\
97 & 47\end{array}$ & 38,240 & 1,830 & 1,000 & 21,000 & 20,000 & - & MPW & 1939 & $\begin{array}{l}\text { Lower Colorado } \\
\text { R1ver Authority }\end{array}$ & - \\
\hline $\begin{array}{l}\text { Lake Balmorhea } \\
\text { Sand1a Creek } \subseteq\end{array}$ & $08-4305$ 8FM & $\begin{array}{rr}30 & 57 \\
103 & 42\end{array}$ & - & 573 & 500 & 6,350 & 5,850 & - & I & 1917 & $\begin{array}{l}\text { Reeves County Water } \\
\text { Improv DIst No } 1\end{array}$ & - \\
\hline $\begin{array}{l}\text { Lake Buffalo Springs } \\
\text { Double Mounta1n Fork Brazos River }\end{array}$ & $08-079550$ & $\begin{array}{rr}33 & 32 \\
101 & 42\end{array}$ & $a 6,000$ & 225 & 0 & 5,360 & 5,360 & 2 & MRW & 1959 & $\begin{array}{l}\text { Lubbock County } \\
\text { Water Improvement } \\
\text { Dist No } 1\end{array}$ & - \\
\hline $\begin{array}{l}\text { Lake Cherokee } \\
\text { Cherokee Bayou }\end{array}$ & $\begin{array}{r}80 \mathrm{~A} \\
08-021500\end{array}$ & $\begin{array}{ll}32 & 23 \\
94 & 38\end{array}$ & 158 & 3,987 & 4,510 & 46,700 & 42,200 & 4 & MRW & 1948 & $\begin{array}{l}\text { Cherokee water co } \\
\text { and city of } \\
\text { Longview }\end{array}$ & $\mathrm{PA}$ \\
\hline $\begin{array}{l}\text { Lake Cisco } \\
\text { Sandy Creek }\end{array}$ & 88-0862 15 & $\begin{array}{ll}32 & 26 \\
98 & 59\end{array}$ & 26 & 1,050 & 0 & 25,600 & 25,600 & - & M & 1923 & C1ty of C1sco & - \\
\hline $\begin{array}{l}\text { Lake Coffee Mill } \\
\text { Corfee Mill Creek }\end{array}$ & $\begin{array}{r}7 \mathrm{LA} \\
07-3326 \quad 10\end{array}$ & $\begin{array}{ll}33 & 44 \\
95 & 59\end{array}$ & 39 & 704 & 0 & 8,000 & 8,000 & 5 & $\mathrm{R}$ & 1938 & Forest Service & - \\
\hline $\begin{array}{l}\text { Lake Colorado C1ty } \\
\text { Morgan Creek }\end{array}$ & $\begin{array}{r}8 \mathrm{LA} \\
08-123000\end{array}$ & $\begin{array}{rr}32 & 19 \\
100 & 55\end{array}$ & 322 & 1,655 & 200 & 31,000 & 30,800 & 4 & MW & 1949 & $\begin{array}{l}\text { Texas Electric } \\
\text { Service Co }\end{array}$ & $\mathrm{PA}$ \\
\hline $\begin{array}{l}\text { Lake Corpus Christi } \\
\text { Nueces River }\end{array}$ & $\mid \begin{array}{r}8 \mathrm{~KB} \\
08-210500\end{array}$ & $\begin{array}{ll}28 & 02 \\
97 & 52\end{array} \mid$ & 16,656 & 15,500 & 140 & 185,900 & 185,800 & 3 & IMRW & 1958 & $\begin{array}{c}\text { Lower Nueces River } \\
\text { Water Supply Dist }\end{array}$ & PA \\
\hline
\end{tabular}


Table 1 --Reservo1rs in the United States completed or under construction as of January 1, 1963, having a usable capac1ty of 5,000 acre-feet or more--Cont1nued

[For meaning of symbols under "Use" and "Storage records publlshed," see text]

\begin{tabular}{|c|c|c|c|c|c|c|c|c|c|c|c|c|}
\hline \multirow[b]{2}{*}{$\begin{array}{l}\text { Name of reservo1r } \\
\text { and } \\
\text { stream }\end{array}$} & \multicolumn{2}{|c|}{ Location } & \multirow{2}{*}{$\begin{array}{l}\text { Drainage } \\
\text { area } \\
\text { (square } \\
\text { m1les) }\end{array}$} & \multirow[b]{2}{*}{$\begin{array}{c}\text { Surface } \\
\text { area } \\
\text { (acres) }\end{array}$} & \multicolumn{3}{|c|}{ Storage in acre-feet } & \multirow[b]{2}{*}{$\begin{array}{c}\text { Storage } \\
\text { rat1o }\end{array}$} & \multirow[b]{2}{*}{ Use } & \multirow[b]{2}{*}{$\begin{array}{c}\text { Date } \\
\text { com- } \\
\text { pleted }\end{array}$} & \multirow[b]{2}{*}{$\begin{array}{l}\text { Owner } \\
\text { or } \\
\text { operator }\end{array}$} & \multirow{2}{*}{$\begin{array}{l}\text { Storage } \\
\text { records } \\
\text { pub- } \\
\text { 21shed }\end{array}$} \\
\hline & \begin{tabular}{|c|} 
Basin \\
1ndex and \\
station no
\end{tabular} & $\begin{array}{l}\text { Lat1 tude } \\
\text { and } \\
\text { long1 tude }\end{array}$ & & & Dead & Total & Usable & & & & & \\
\hline TEXAS--ContInued & & & & & & & & & & & & \\
\hline $\begin{array}{l}\text { Lake Nasworthy } \\
\text { South Concho River }\end{array}$ & $08-1320^{8 \mathrm{LB}}$ & $\begin{array}{rr}31 & 23 \\
100 & 29\end{array}$ & 3,833 & 1,596 & o & 12,390 & 12,390 & 02 & IMW & 1930 & City of San Angelo & PA \\
\hline $\begin{array}{l}\text { Lake o' the Pines } \\
\text { Cypress Creek }\end{array}$ & $07-3459$ OMA & $\begin{array}{ll}32 & 45 \\
94 & 30\end{array}$ & 850 & 38,200 & 2,860 & 842,100 & 839,240 & 18 & FMR & 1957 & Corps of Eng1neers & PA \\
\hline $\begin{array}{l}\text { Lake Palestine (Blackburn Crossing) } \\
\text { Neches River }\end{array}$ & 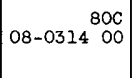 & $\begin{array}{ll}32 & 03 \\
95 & 26\end{array}$ & 839 & 6,800 & 550 & 57,600 & 57,000 & 1 & MW & 1962 & $\begin{array}{l}\text { Upper Neches River } \\
\text { Municipal Water } \\
\text { Authority }\end{array}$ & PA \\
\hline $\begin{array}{l}\text { Lake Quitman } \\
\text { Dry Creek }\end{array}$ & $08-0189^{80 \mathrm{~A}}$ & $\begin{array}{ll}32 & 51 \\
95 & 27\end{array}$ & 31 & 814 & of & 7,440 & 7,440 & 5 & $\mathrm{FR}$ & 1962 & Wood County & - \\
\hline $\begin{array}{l}\text { Lake Randall } \\
\text { Shawnee Creek c/ }\end{array}$ & $07-3316 \quad 20$ & $\begin{array}{ll}33 & 48 \\
96 & 35\end{array}$ & - & 172 & 0 & 5,400 & 5,400 & - & M & 1909 & C1ty of Dentson & - \\
\hline $\begin{array}{l}\text { Lake Rita Blanca } \\
\text { R1ta Blanca Creek }\end{array}$ & $07-2274 \quad 40$ & $\begin{array}{rr}36 & 02 \\
102 & 30\end{array}$ & 1,062 & 524 & 0 & 12,100 & 12,100 & 5 & $\mathrm{R}$ & 1939 & $\begin{array}{l}\text { So1l Conservation } \\
\text { Service }\end{array}$ & - \\
\hline $\begin{array}{l}\text { Lake Stamford } \\
\text { Paint Creek }\end{array}$ & $08-084500$ & $\begin{array}{ll}33 & 05 \\
99 & 35\end{array}$ & 360 & 5,125 & 430 & 60,000 & 59,600 & 3 & $M w$ & 1953 & C1ty of Stamford & PA \\
\hline $\begin{array}{l}\text { Lake Sweetwater } \\
\text { Bitterwood and Cottonwood Creeks }\end{array}$ & $08-0832 \begin{array}{rr}8 \mathrm{MB} \\
00\end{array}$ & $\begin{array}{rr}32 & 26 \\
100 & 18\end{array}$ & 104 & 630 & 0 & 11,900 & 11,900 & 2 & MW & 1930 & C1ty of Sweetwater & PA \\
\hline $\begin{array}{l}\text { Lake Tawakon1 } \\
\text { Sab1ne R1ver }\end{array}$ & $\begin{array}{r}80 \mathrm{~A} \\
08-017400\end{array}$ & $\begin{array}{ll}32 & 49 \\
95 & 55\end{array}$ & 756 & 36,700 & 0 & 936,200 & 936,200 & 26 & IMW & 1960 & $\begin{array}{l}\text { Sab1ne River } \\
\text { Authorlty }\end{array}$ & PA \\
\hline $\begin{array}{l}\text { Lake Texoma (Denison Dam)* } \\
\text { Red River }\end{array}$ & $07-3315$ 7LA & $\begin{array}{ll}33 & 49 \\
96 & 34\end{array}$ & 39,719 & 144,100 & $\mathrm{~b} 1,106,000$ & $5,530,300$ & $4,424,300$ & 11 & FMPRW & 1943 & Corps of Engineers & PA \\
\hline $\begin{array}{l}\text { Lake Travis (Marshall Ford Dam) } \\
\text { Colorado River }\end{array}$ & $08-1545^{8 L F}$ & $\begin{array}{ll}30 & 23 \\
97 & 55\end{array}$ & 38,130 & 29,000 & 27,900 & $1,950,000$ & $1,922,000$ & 10 & FIMPRW & 1942 & $\begin{array}{l}\text { Lower Colorado } \\
\text { R1ver Authority }\end{array}$ & $\mathrm{PA}$ \\
\hline $\begin{array}{l}\text { Lake Tyler } \\
\text { Prairie Creek }\end{array}$ & $\begin{array}{r}800 \\
08-034000\end{array}$ & $\begin{array}{ll}32 & 13 \\
95 & 10\end{array}$ & 453 & 2,450 & 4,400 & 43,400 & 39,000 & 18 & MRW & 1949 & C1ty of Tyler & PA \\
\hline $\begin{array}{l}\text { Lake Waco } \\
\text { Bosque River }\end{array}$ & $08-0955$ 8MC & $\begin{array}{ll}31 & 34 \\
97 & 12\end{array}$ & 1,651 & 2,742 & 0 & 22,030 & 22,030 & 08 & $M W$ & 1930 & City of Waco & - \\
\hline $\begin{array}{l}\text { Lake Walk } \\
\text { Devils River }\end{array}$ & $08-449480$ & $\begin{array}{rr}29 & 30 \\
100 & 59\end{array}$ & 3,923 & 380 & 0 & 5,400 & 5,400 & $<05$ & $P$ & 1929 & $\begin{array}{l}\text { Centra1 Power and } \\
\text { L1ght Co }\end{array}$ & - \\
\hline $\begin{array}{l}\text { Lake Waxahachie } \\
\text { South Prong Waxahach1e Creek }\end{array}$ & O8-0643 800 & $\begin{array}{ll}32 & 20 \\
96 & 48\end{array}$ & 30 & 645 & 1,500 & 13,500 & 12,000 & 15 & MW & 2956 & $\begin{array}{l}\text { Ellis County Water } \\
\text { Control and } \\
\text { Improv Dist No } 1\end{array}$ & - \\
\hline
\end{tabular}




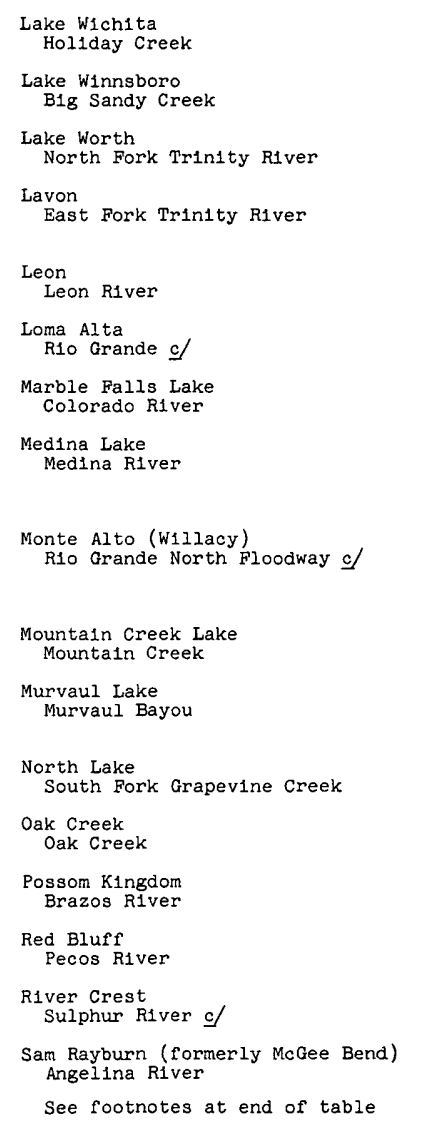

\begin{tabular}{|c|c|c|c|}
\hline $\begin{array}{r}7 \mathrm{KC} \\
07-3126 \quad 00\end{array}$ & $\begin{array}{ll}33 & 52 \\
98 & 31\end{array}$ & 143 & 2,200 \\
\hline $08-0193$ OOA & $\begin{array}{ll}32 & 53 \\
95 & 21\end{array}$ & 31 & 720 \\
\hline $\begin{array}{r}8 \mathrm{NB} \\
08-0454 \quad 00\end{array}$ & $\begin{array}{ll}32 & 49 \\
97 & 25\end{array}$ & 2,064 & 3,267 \\
\hline $\begin{array}{r}8 \mathrm{NB} \\
08-0605 \quad 00\end{array}$ & $\begin{array}{ll}33 & 01 \\
96 & 29\end{array} \mid$ & 770 & 20,050 \\
\hline $\begin{array}{r}8 \mathrm{MD} \\
08-0990 \quad 00\end{array}$ & $\begin{array}{ll}32 & 21 \\
98 & 41\end{array}$ & 259 & 1,590 \\
\hline $08-4742 \quad 00$ & $\begin{array}{ll}25 & 59 \\
57 & 25\end{array}$ & - & 2,490 \\
\hline $08-1483^{8 L D}$ & $\begin{array}{ll}30 & 33 \\
98 & 16\end{array}$ & 36,325 & 780 \\
\hline 8KF & $\begin{array}{ll}29 & 32 \\
98 & 56\end{array}$ & 609 & 5,575 \\
\hline $08-4735 \stackrel{8 \mathrm{~J}}{50}$ & $\begin{array}{ll}26 & 25 \\
97 & 58\end{array}$ & - & 2,371 \\
\hline $08-0500^{8 \mathrm{NB}}$ & $\begin{array}{ll}32 & 44 \\
96 & 57\end{array}$ & 295 & 2,940 \\
\hline $08-0222^{80 A}$ & $\begin{array}{ll}32 & 02 \\
94 & 25\end{array}$ & 115 & 3,820 \\
\hline $\begin{array}{r}8 \mathrm{NB} \\
08-055550\end{array}$ & $\begin{array}{ll}32 & 57 \\
96 & 57\end{array}$ & 3 & 820 \\
\hline $08-1255^{8 L A}$ 00 & $\begin{array}{rr}32 & 04 \\
100 & 17\end{array}$ & 244 & 2,375 \\
\hline $\begin{array}{r}8 M C \\
08-088500\end{array}$ & $\begin{array}{ll}32 & 52 \\
98 & 26\end{array}$ & 22,550 & 19,800 \\
\hline $08-4100 \quad 8 \mathrm{FL}$ & $\begin{array}{rr}31 & 54 \\
103 & 55\end{array}$ & $\mathrm{a} 20,700$ & 11,700 \\
\hline $\begin{array}{r}7 M C \\
07-343100\end{array}$ & $\begin{array}{ll}32 & 24 \\
95 & 09\end{array}$ & - & 560 \\
\hline$-80 c$ & $\begin{array}{ll}31 & 04 \\
94 & 05\end{array}$ & 3,449 & 114,500 \\
\hline
\end{tabular}
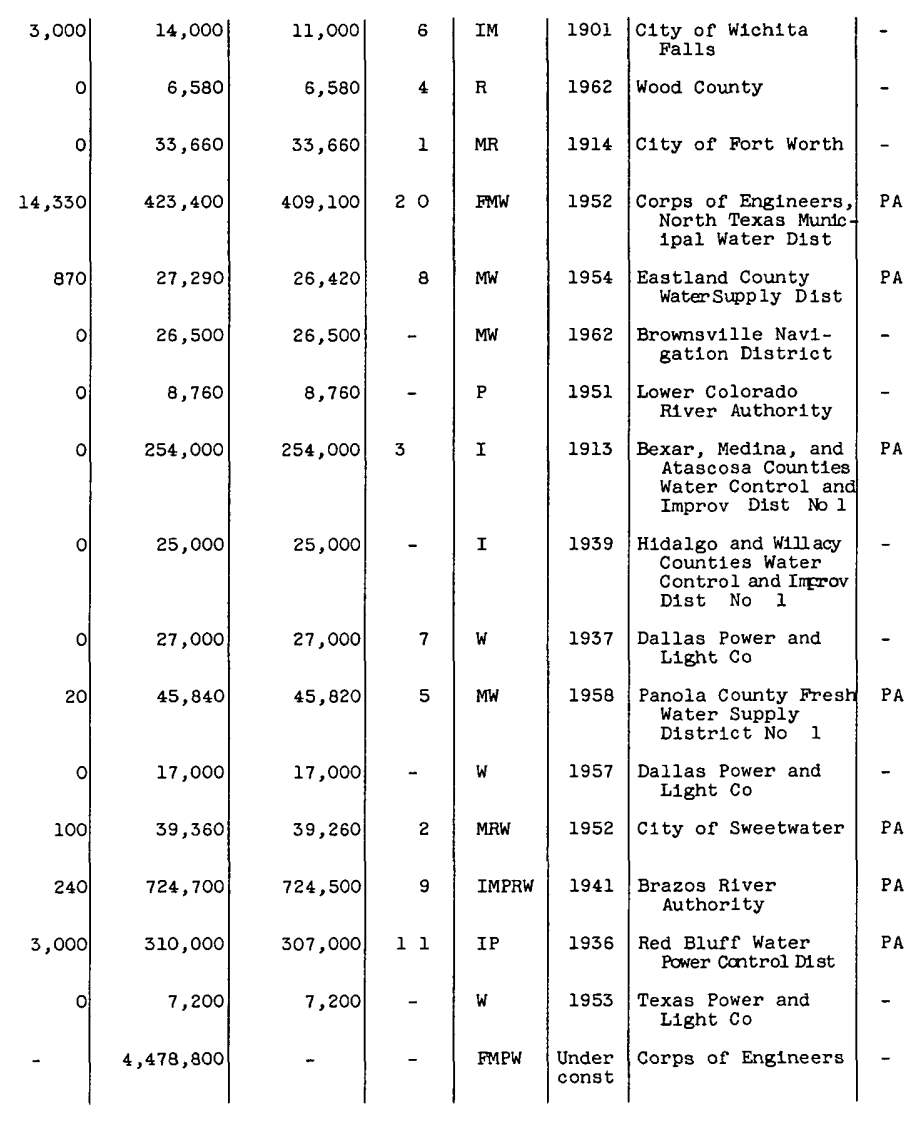
Table 1 --Reservo1rs in the United States completed or under construction as of January 1, 1963, having a usable capac1ty of 5,000 acre-feet or more--Continued

\begin{tabular}{|c|c|c|c|c|c|c|c|c|c|c|c|c|}
\hline \multirow[b]{2}{*}{$\begin{array}{l}\text { Name of reservoir } \\
\text { and } \\
\text { stream }\end{array}$} & \multicolumn{2}{|c|}{ Location } & \multirow{2}{*}{$\begin{array}{l}\text { Drainage } \\
\text { area } \\
\text { (square } \\
\text { miles) }\end{array}$} & \multirow[b]{2}{*}{$\begin{array}{c}\text { Surface } \\
\text { area } \\
\text { (acres) }\end{array}$} & \multicolumn{3}{|c|}{ Storage in acne-feet } & \multirow[b]{2}{*}{\begin{tabular}{|} 
Storage \\
rat1o
\end{tabular}} & \multirow[b]{2}{*}{ Use } & \multirow[b]{2}{*}{$\begin{array}{c}\text { Date } \\
\text { com- } \\
\text { pleted }\end{array}$} & \multirow[b]{2}{*}{$\begin{array}{l}\text { Owner } \\
\text { or } \\
\text { operator }\end{array}$} & \multirow{2}{*}{$\begin{array}{c}\text { Storage } \\
\text { records } \\
\text { pub- } \\
\text { l1shed }\end{array}$} \\
\hline & \begin{tabular}{|c|} 
Basin \\
1ndex and \\
station no \\
\end{tabular} & $\begin{array}{l}\text { Lat1 tude } \\
\text { and } \\
\text { long1 tude }\end{array}$ & & & Dead & Total & Usable & & & & & \\
\hline TEXAS--Continued & & & & & & & & & & & & \\
\hline $\begin{array}{l}\text { San Angelo } \\
\text { North Concho River }\end{array}$ & $\mid \begin{array}{r}8 \mathrm{LB} \\
08-1345 \quad 00\end{array}$ & $\begin{array}{rr}31 & 29 \\
100 & 29\end{array}$ & 1,488 & 12,700 & 0 & 396,400 & 396,400 & - & FIMR & 1951 & $\begin{array}{l}\text { Corps of Engineers, } \\
\text { Upper Colorado } \\
\text { River Author1ty }\end{array}$ & $\mathrm{PA}$ \\
\hline $\begin{array}{l}\text { San Estaban Lake } \\
\text { Alami to Creek }\end{array}$ & $08-373900$ & $\begin{array}{rr}30 & 10 \\
104 & 02\end{array}$ & 461 & 762 & 0 & 18,770 & 18,770 & 3 & I & 1911 & $\begin{array}{l}\text { Mrs Pearle M Rob- } \\
\text { Inson Estate }\end{array}$ & - \\
\hline $\begin{array}{l}\text { Santa Rosa Lake } \\
\text { Beaver Creek }\end{array}$ & $\begin{array}{r}7 \mathrm{KC} \\
07-312150\end{array}$ & $\begin{array}{ll}33 & 56 \\
99 & 15\end{array}$ & 336 & al, 500 & 0 & al1,570 & al1, 570 & 5 & IM & 1929 & $\begin{array}{l}\text { W Waggoner } \\
\text { Estate }\end{array}$ & - \\
\hline $\begin{array}{l}\text { Sheldon } \\
\text { Carpenters Bayou }\end{array}$ & 8NA & $\begin{array}{ll}29 & 52 \\
95 & 10\end{array}$ & 9 & 1,200 & 0 & 5,420 & 5,420 & - & - & 1943 & $\begin{array}{l}\text { Texas Game and Fish } \\
\text { Comm }\end{array}$ & - \\
\hline $\begin{array}{l}\text { Smithers Lake (Lake George) } \\
\text { Dry Creek }\end{array}$ & $08-1166 \quad 8 M 0$ & $\begin{array}{ll}29 & 29 \\
95 & 38\end{array}$ & 242 & 2,140 & 0 & 28,000 & 18,000 & - & W & 1957 & $\begin{array}{l}\text { Houston Light and } \\
\text { Power Co }\end{array}$ & - \\
\hline $\begin{array}{l}\text { Striker Creek } \\
\text { Striker Creek }\end{array}$ & $08-033800$ & $\begin{array}{ll}31 & 56 \\
94 & 59\end{array}$ & 182 & 2,400 & o & 26,700 & 26,700 & 2 & MW & 1957 & $\begin{array}{c}\text { Angelina-Nacogioches } \\
\text { Water Control and } \\
\text { Improv Dist No } 1\end{array}$ & - \\
\hline $\begin{array}{l}\text { Terrell } \\
\text { Muddy Cedar Creek }\end{array}$ & $\begin{array}{r}8 \mathrm{NC} \\
08-062740\end{array}$ & $\begin{array}{ll}32 & 44 \\
96 & 11\end{array}$ & 14 & 885 & 0 & 8,300 & 8,300 & 2 & MR & 1955 & City of Terrell & - \\
\hline $\begin{array}{l}\text { Texarkana } \\
\text { Sulphur River }\end{array}$ & 07-3442 00 & $\begin{array}{ll}33 & 18 \\
94 & 10\end{array}$ & 3,443 & 119,700 & 0 & $2,654,300$ & $2,654,300$ & 11 & CFM & $\begin{array}{l}1957 \\
-\end{array}$ & Corps of Engineers & PA \\
\hline $\begin{array}{l}\text { Town Bluff Lake (Dam B) } \\
\text { Neches River }\end{array}$ & $\mid \begin{array}{r}800 \\
08-040000\end{array}$ & $\begin{array}{ll}30 & 48 \\
94 & 11\end{array}$ & 7,573 & 16,830 & o & 124,700 & 124,700 & 4 & IMRW & 1951 & Corps of Engineers & PA \\
\hline $\begin{array}{l}\text { Tranquitas } \\
\text { Tranquitas Creek }\end{array}$ & 08-212200 & $\begin{array}{ll}27 & 34 \\
97 & 56\end{array}$ & - & 400 & 0 & 6,000 & 6,000 & - & $\mathrm{C}$ & - & King Ranch & - \\
\hline $\begin{array}{l}\text { Trinidad Lake } \\
\text { Trinity River } c\end{array}$ & 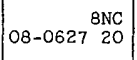 & $\begin{array}{ll}32 & 07 \\
96 & 05\end{array}$ & - & 753 & 0 & 7,800 & 7,800 & - & w & 1925 & $\begin{array}{l}\text { Texas Power and } \\
\text { Light Co }\end{array}$ & - \\
\hline $\begin{array}{l}\text { Twin Buttes } \\
\text { Middle and South Concho Rivers }\end{array}$ & $\mid 08-1312$ 8L.B & $\begin{array}{rr}31 & 23 \\
100 & 32\end{array}$ & 3,724 & 22,700 & 5,100 & 600,000 & 594,900 & - & FIMN & 1962 & $\begin{array}{l}\text { C1ty of San Angelo, } \\
\text { Bureau of } \\
\text { Reclamation }\end{array}$ & PA \\
\hline $\begin{array}{l}\text { Upper Nueces } \\
\text { Nueces RIver }\end{array}$ & 08-1922 $8 \mathrm{~KB}$ & $\begin{array}{ll}28 & 48 \\
99 & 49\end{array}$ & a2, 160 & 316 & 0 & 7,590 & 7,590 & $<05$ & I & 1947 & \begin{tabular}{|l|} 
Zavala-Dimmit \\
Count1es Water \\
Control and \\
Improv Dist No 1
\end{tabular} & - \\
\hline $\begin{array}{l}\text { Valley Acres } \\
\text { Rio Grande North Floodway } c^{\prime}\end{array}$ & $08-4736$ OJ & $\begin{array}{ll}26 & 15 \\
97 & 52\end{array}$ & - & 906 & 0 & 7,840 & 7,840 & - & I & 1951 & $\begin{array}{l}\text { Valley Acres water } \\
\text { District }\end{array}$ & - \\
\hline
\end{tabular}


Victor Braunig Lake
Arroyo Seco

Weatherford Lake
Clear Fork Trinity River

White Rock Lake

Whitney
Brazos River

William Harris

UTAH

Ash Creek

Cutler
Bear River

Deer Creek

Desert Lake (Farmers Dam)

Hunt1ngton Creek $\mathrm{c}$ /

DMAD

Sevier River $c$

East Canyon

East Canyon
East Canyon Creek

Echo

Weber River

Flaming Gorge
Green River

Fremont (Mill Meadow)
Fremont River

Gunnison

Pitch River

Hyrum
Little Bear River

Johnson

Fremont River

Kolob

Kolob Creek

See footnotes at end of table

\begin{tabular}{|c|c|c|}
\hline $08-1817 \stackrel{8 K F}{00}$ & $\begin{array}{ll}29 & 15 \\
98 & 22\end{array}$ & - \\
\hline $08-0457^{8 N B}$ & $\begin{array}{ll}32 & 46 \\
97 & 40\end{array}$ & 109 \\
\hline $08-057300$ & $\begin{array}{ll}32 & 49 \\
96 & 43\end{array}$ & 100 \\
\hline $\begin{array}{r}8 M C \\
08-092500\end{array}$ & $\begin{array}{ll}31 & 52 \\
97 & 22\end{array}$ & 26,170 \\
\hline $08-116800$ & $\begin{array}{ll}29 & 14 \\
95 & 34\end{array}$ & - \\
\hline $09-4064 \stackrel{9 K}{0}$ & $\begin{array}{rr}37 & 25 \\
113 & 14\end{array}$ & - \\
\hline $\begin{array}{r}10 \mathrm{HC} \\
10-116500\end{array}$ & $\begin{array}{rr}41 & 50 \\
112 & 03\end{array}$ & 6,000 \\
\hline $\begin{array}{r}10 \mathrm{HG} \\
10-159000\end{array}$ & $\begin{array}{rr}40 & 24 \\
111 & 32\end{array}$ & 560 \\
\hline $09-3143^{9 \mathrm{BJ}} 00$ & $\begin{array}{rr}39 & 22 \\
110 & 47\end{array}$ & - \\
\hline $\begin{array}{r}10 J E \\
10-224500\end{array}$ & $\begin{array}{rr}39 & 24 \\
112 & 29\end{array}$ & - \\
\hline $\begin{array}{r}10 \mathrm{OHE} \\
10-134000\end{array}$ & $\begin{array}{rr}40 & 55 \\
111 & 36\end{array}$ & 155 \\
\hline $\begin{array}{r}10 \mathrm{HE} \\
10-131500\end{array}$ & $\begin{array}{rr}40 & 58 \\
111 & 26\end{array}$ & 732 \\
\hline $\begin{array}{r}9 \mathrm{AK} \\
09-234400\end{array}$ & $\begin{array}{rr}40 & 55 \\
109 & 25\end{array}$ & 15,100 \\
\hline $09-3294900$ & $\begin{array}{rr}38 & 30 \\
111 & 34\end{array}$ & 203 \\
\hline 10-216200 & $\begin{array}{rr}39 & 13 \\
111 & 42\end{array}$ & a720 \\
\hline $\begin{array}{r}10 \mathrm{HC} \\
10-107000\end{array}$ & $\begin{array}{rr}41 & 38 \\
111 & 52\end{array}$ & 220 \\
\hline $09-329100$ & $\begin{array}{rr}38 & 36 \\
111 & 38\end{array}$ & $a 60$ \\
\hline $09-405400$ & $\begin{array}{rr}37 & 26 \\
113 & 02\end{array}$ & - \\
\hline
\end{tabular}

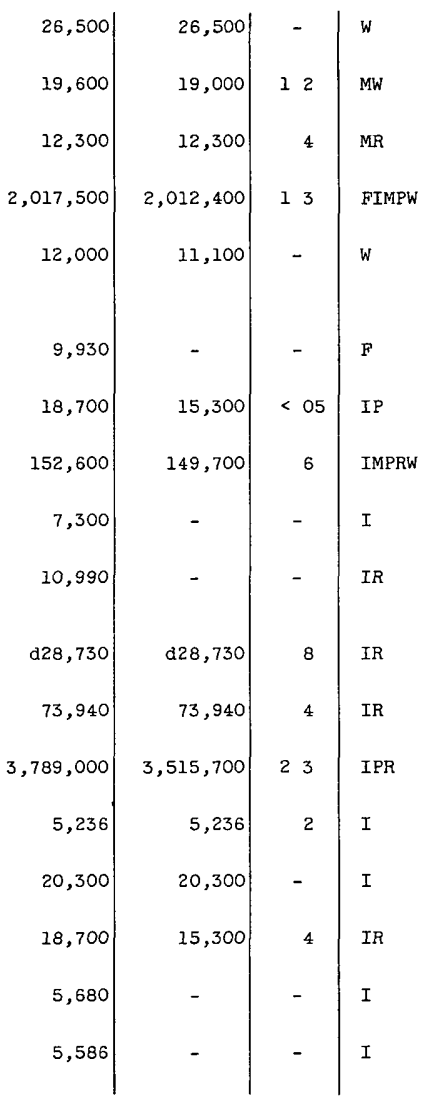


Table 1 --Reservo1rs in the Un1ted States completed or under construction as of January 1, 1963, having a usable capac1ty of 5,000 acre-feet or more--Cont1nued [For meaning of symbols under "Use" and "Storage records published," see text]

\begin{tabular}{|c|c|c|c|c|c|c|c|c|c|c|c|c|}
\hline \multirow[b]{2}{*}{$\begin{array}{l}\text { Name of reservo1r } \\
\text { and } \\
\text { stream }\end{array}$} & \multicolumn{2}{|c|}{ Location } & \multirow{2}{*}{$\begin{array}{l}\text { Drainage } \\
\text { area } \\
\text { (square } \\
\text { m1les) }\end{array}$} & \multirow[b]{2}{*}{$\begin{array}{c}\text { Surface } \\
\text { area } \\
\text { (acres) }\end{array}$} & \multicolumn{3}{|c|}{ Storage in acre-feet } & \multirow[b]{2}{*}{ 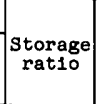 } & \multirow[b]{2}{*}{ Use } & \multirow[b]{2}{*}{$\begin{array}{c}\text { Date } \\
\text { com- } \\
\text { pleted }\end{array}$} & \multirow[b]{2}{*}{$\begin{array}{l}\text { Owner } \\
\text { or } \\
\text { operator }\end{array}$} & \multirow{2}{*}{$\begin{array}{l}\text { Storage } \\
\text { records } \\
\text { pub- } \\
\text { lished }\end{array}$} \\
\hline & \begin{tabular}{|c|} 
Basin \\
1ndex and \\
station no
\end{tabular} & $\begin{array}{l}\text { Lat1 tude } \\
\text { and } \\
\text { long1tude }\end{array}$ & & & Dead & Total & Usable & & & & & \\
\hline UTAH--Cont1nued & & & & & & & & & & & & \\
\hline $\begin{array}{l}\text { Mantua } \\
\text { B1g Creek }\end{array}$ & $\begin{array}{r}10 \mathrm{HC} \\
10-126450\end{array}$ & \begin{tabular}{rr|r}
41 & 30 \\
111 & 56
\end{tabular} & - & 554 & - & 7,560 & - & - & IMPR & 1961 & Brigham C1ty, Corp & - \\
\hline $\begin{array}{l}\text { Lake Bouram (M1dview Dam and D1ke) } \\
\text { Duchesne River } \underline{\mathrm{c}}\end{array}$ & $09-2887$ 90 & \begin{tabular}{rr|}
40 & 11 \\
110 & 10
\end{tabular} & - & 405 & 0 & 5,800 & 5,800 & - & I & 1937 & $\begin{array}{l}\text { Moon Lake water } \\
\text { Users Issoc }\end{array}$ & - \\
\hline $\begin{array}{l}\text { Millers Flat } \\
\text { Millers Flat Creek }\end{array}$ & $09-3177^{9 B K}$ & $\begin{array}{rr}39 & 33 \\
111 & 14\end{array}$ & - & 200 & - & 6,765 & - & - & IR & 1943 & $\begin{array}{l}\text { Huntington, Cleve- } \\
\text { land Irrig Co }\end{array}$ & - \\
\hline $\begin{array}{l}\text { Mona (Mount Nebo Dam) } \\
\text { Currant Creek }\end{array}$ & $\begin{array}{r}10 \mathrm{HF} \\
10-146400\end{array}$ & $\begin{array}{rr}39 & 53 \\
111 & 52\end{array}$ & - & 1,620 & - & 19,000 & - & - & I & 1895 & $\begin{array}{l}\text { Mount Nebo Irriga- } \\
\text { tion Co }\end{array}$ & - \\
\hline $\begin{array}{l}\text { Moon Lake } \\
\text { Lake Fork }\end{array}$ & $09-2905$ 9BC & $\begin{array}{rr}40 & 34 \\
110 & 30\end{array}$ & 108 & 773 & 13,740 & 49,500 & 35,800 & 04 & IPR & 1938 & $\begin{array}{l}\text { Moon Lake Water } \\
\text { Users Assoc }\end{array}$ & PA \\
\hline $\begin{array}{l}\text { Navajo Lake } \\
\text { Duck Creek }\end{array}$ & $\begin{array}{r}10 J A \\
10-173700\end{array}$ & $\begin{array}{rr}37 & 31 \\
112 & 46\end{array}$ & - & 618 & - & 14,870 & - & - & $R$ & 1940 & $\begin{array}{l}\text { St George, Washing } \\
\text { ton, Hurricane, } \\
\text { and LaVerkin } \\
\text { Canal Cos }\end{array}$ & - \\
\hline $\begin{array}{l}\text { Neponset } \\
\text { Bear River } c /\end{array}$ & $10-021200$ & \begin{tabular}{rr|}
41 & 23 \\
111 & 07
\end{tabular} & - & 1,043 & - & 8,701 & - & - & I & 1924 & $\begin{array}{l}\text { Deseret L1vestock } \\
\text { Co }\end{array}$ & - \\
\hline $\begin{array}{l}\text { Newcastie (P1nto Creek) } \\
\text { P1nto Creek c/ }\end{array}$ & $10-242500$ & $\begin{array}{rr}37 & 39 \\
113 & 32\end{array}$ & - & - & - & 5,290 & - & - & IR & 1954 & $\underset{\text { Co }}{\text { Newcastle Reservo1r }}$ & - \\
\hline $\begin{array}{l}\text { Newton } \\
\quad \text { Clarkston Creek }\end{array}$ & $10-1154$ OO & $\begin{array}{rr}41 & 54 \\
111 & 59\end{array}$ & 233 & 297 & 226 & 5,600 & 5,370 & - & IM & 1944 & $\begin{array}{l}\text { Bureau of } \\
\text { Reclamation }\end{array}$ & - \\
\hline $\begin{array}{l}\text { Oaks Park (Buck Pasture Dam) } \\
\text { Brush Creek }\end{array}$ & $09-2649$ OBA & $\begin{array}{rr}40 & 45 \\
109 & 37\end{array}$ & 102 & 382 & of & 6,250 & 6,250 & 5 & I & 1940 & $\begin{array}{l}\text { Ashley Valley } \\
\text { Reservo1r Co }\end{array}$ & - \\
\hline $\begin{array}{l}\text { Otter Creek } \\
\text { Otter Creek and East Fork Sevier } \\
\text { R1ver } \mathrm{c} /\end{array}$ & $\begin{array}{r}10 J 8 \\
10-188000\end{array}$ & $\begin{array}{rr}38 & 10 \\
112 & 00\end{array}$ & - & 2,500 & 0 & 52,500 & 52,500 & - & I & 1891 & $\begin{array}{l}\text { otter Creek Reser- } \\
\text { volr Co }\end{array}$ & PA \\
\hline $\begin{array}{l}\text { Panguitch Lake } \\
\text { Panguitch Creek }\end{array}$ & $\begin{array}{r}10 J A \\
10-1762 \text { OO }\end{array}$ & $\begin{array}{rr}37 & 44 \\
112 & 38\end{array}$ & 67 & 1,226 & 777 & 18,600 & 17,800 & 10 & IR & 1872 & $\begin{array}{l}\text { West Panguitch Res- } \\
\text { ervo1r and Irriga- } \\
\text { tion Co }\end{array}$ & - \\
\hline $\begin{array}{l}\text { Pine View } \\
\text { Ogden R1ver }\end{array}$ & $\begin{array}{r}10 \mathrm{HE} \\
10-1390 \text { OO }\end{array}$ & $\begin{array}{rr}41 & 15 \\
111 & 50\end{array}$ & a310 & 2,870 & 45 & 110,100 & 110,100 & 2 & IR & 1936 & $\begin{array}{l}\text { Bureau of } \\
\text { Reclamation }\end{array}$ & $\mathrm{PA}$ \\
\hline $\begin{array}{l}\text { Plute } \\
\text { Sevier River }\end{array}$ & $\begin{array}{r}10 \mathrm{JC} \\
10-191000\end{array}$ & $\begin{array}{rr}38 & 19 \\
112 & 11\end{array}$ & a2, 400 & 2,250 & 0 & 74,010 & 74,010 & 5 & I & 1910 & Piute Reservo1r Co & $\mathrm{PA}$ \\
\hline
\end{tabular}




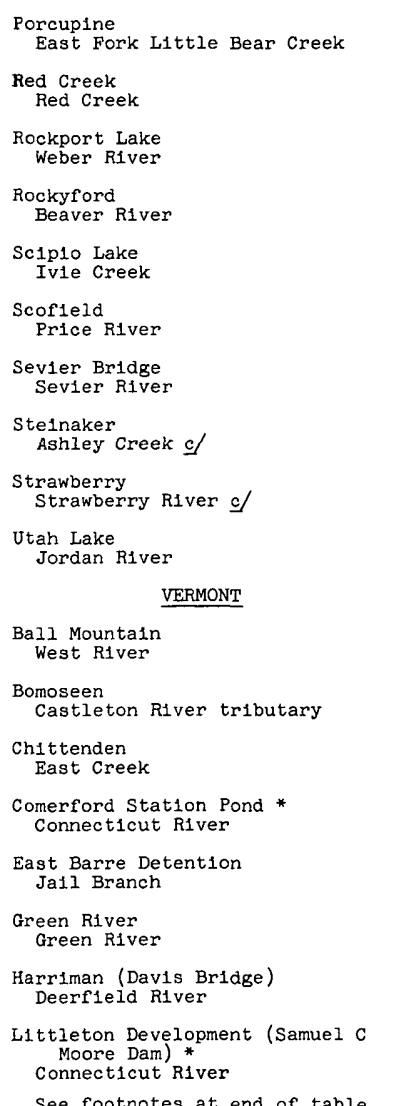

\begin{tabular}{|c|c|c|c|c|c|}
\hline $10-105200$ & \begin{tabular}{rr|}
41 & 31 \\
111 & 45
\end{tabular} & - & 220 & - & 12,695 \\
\hline $09-286191^{9 B B}$ & $\begin{array}{rr}40 & 18 \\
110 & 51\end{array}$ & 39 & 140 & - & 5,700 \\
\hline $\begin{array}{r}10 \mathrm{HE} \\
10-129400\end{array}$ & $\begin{array}{rr}40 & 47 \\
111 & 24\end{array}$ & 320 & 1,080 & 1,260 & 62,120 \\
\hline $\begin{array}{r}10 \mathrm{JF} \\
10-238500\end{array}$ & $\begin{array}{rr}38 & 13 \\
112 & 50\end{array}$ & as10 & 1,129 & of & 23,260 \\
\hline $\begin{array}{r}10 \mathrm{JF} \\
10-2190 \quad 30\end{array}$ & $\begin{array}{rr}39 & 07 \\
112 & 03\end{array}$ & a.105 & 1,400 & 630 & 9,500 \\
\hline $09-3110900$ & $\begin{array}{rr}39 & 47 \\
111 & 08\end{array}$ & 163 & 2,810 & 8,000 & 73,780 \\
\hline $\begin{array}{r}\text { 10JE } \\
10-218500\end{array}$ & $\begin{array}{rr}39 & 22 \\
112 & 02\end{array}$ & 25,120 & 10,700 & 0 & 236,000 \\
\hline $09-2713 \stackrel{9 B E}{00}$ & $\begin{array}{rr}40 & 30 \\
109 & 32\end{array}$ & - & 820 & 3,720 & 37,560 \\
\hline $\begin{array}{r}9 \mathrm{9B} \\
09-2825 \quad 00\end{array}$ & $\begin{array}{rr}40 & 10 \\
110 & 11\end{array}$ & - & 8,400 & 13,000 & 257,600 \\
\hline $\begin{array}{r}10 \mathrm{HF} \\
10-166500\end{array}$ & $\begin{array}{rr}40 & 22 \\
111 & 54\end{array}$ & 2,570 & 95,900 & 22,300 & 850,200 \\
\hline $01-1554$ OO & $\begin{array}{ll}43 & 08 \\
72 & 46\end{array}$ & 172 & 810 & - & - \\
\hline $\begin{array}{r}4 P D \\
04-279950\end{array}$ & $\begin{array}{ll}43 & 36 \\
73 & 14\end{array}$ & 27 & - & - & - \\
\hline $\begin{array}{r}4 \mathrm{PF} \\
04-280980\end{array}$ & $\begin{array}{ll}43 & 43 \\
72 & 55\end{array}$ & 14 & - & - & - \\
\hline $\begin{array}{r}\text { IGB } \\
01-132500\end{array}$ & $\begin{array}{ll}44 & 20 \\
72 & 00\end{array}$ & 1,635 & 1,093 & 23,620 & 52,976 \\
\hline $\begin{array}{r}4 P G \\
04-283500\end{array}$ & $\begin{array}{ll}44 & 09 \\
72 & 27\end{array}$ & 388 & 665 & 112 & 11,800 \\
\hline $\begin{array}{r}4 \mathrm{PH} \\
04-290850\end{array}$ & $\begin{array}{ll}44 & 37 \\
72 & 32\end{array}$ & 14 & - & - & 17,500 \\
\hline $01-1680^{1 G L}$ & $\begin{array}{ll}42 & 48 \\
72 & 54\end{array}$ & 184 & 2,184 & 16,775 & 132,850 \\
\hline $\begin{array}{r}1 \mathrm{~GB} \\
01-1320 \text { 00 }\end{array}$ & $\begin{array}{ll}44 & 20 \\
71 & 52\end{array}$ & 1,600 & 3,490 & 109,676 & 223,852 \\
\hline
\end{tabular}

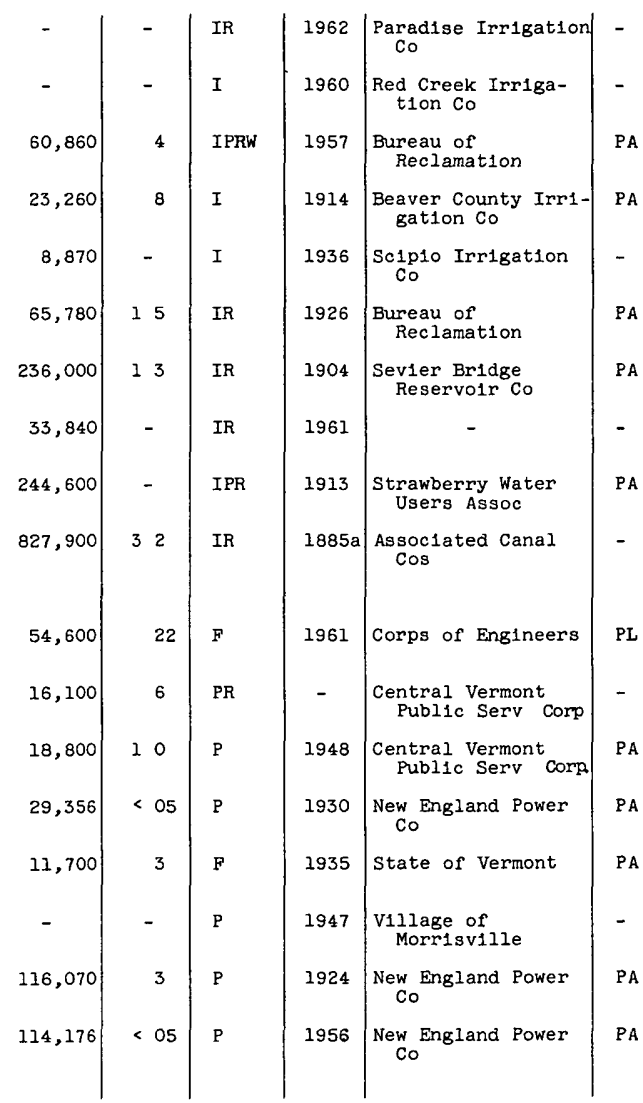



[For meaning of symbols under "Use" and "Storage records published," see text]

\begin{tabular}{|c|c|c|c|c|c|c|c|c|c|c|c|c|}
\hline \multirow[b]{2}{*}{$\begin{array}{l}\text { Name of reservo1r } \\
\text { and } \\
\text { stream }\end{array}$} & \multicolumn{2}{|c|}{ Location } & \multirow{2}{*}{$\begin{array}{l}\text { Drainage } \\
\text { area } \\
\text { (square } \\
\text { m1les) }\end{array}$} & \multirow[b]{2}{*}{$\begin{array}{c}\text { Surface } \\
\text { area } \\
\text { (acres) }\end{array}$} & \multicolumn{3}{|c|}{ Storage in acre-feet } & \multirow[b]{2}{*}{$\begin{array}{c}\text { Storage } \\
\text { rat10 }\end{array}$} & \multirow[b]{2}{*}{ Use } & \multirow[b]{2}{*}{$\begin{array}{c}\text { Date } \\
\text { com- } \\
\text { pleted }\end{array}$} & \multirow[b]{2}{*}{$\begin{array}{l}\text { Owner } \\
\text { or } \\
\text { operator }\end{array}$} & \multirow{2}{*}{$\begin{array}{l}\text { Storage } \\
\text { records } \\
\text { pub- } \\
\text { lished }\end{array}$} \\
\hline & \begin{tabular}{|c|} 
Basin \\
1ndex and \\
station no
\end{tabular} & $\begin{array}{l}\text { Lat1 tude } \\
\text { and } \\
\text { long1tude }\end{array}$ & & & Dead & Total & Usable & & & & & \\
\hline VERMONT--Continued & & & & & & & & & & & & \\
\hline $\begin{array}{l}\text { Mollys Falls } \\
\text { Mollys Brook }\end{array}$ & $\begin{array}{r}4 P G \\
04-2829,50\end{array}$ & $\begin{array}{ll}44 & 22 \\
72 & 18\end{array}$ & 23 & 414 & - & - & 9,105 & 03 & $P$ & 1926 & $\begin{array}{l}\text { Green Mountain } \\
\text { Power Corp }\end{array}$ & $\mathrm{PA}$ \\
\hline $\begin{array}{l}\text { North Hartland } \\
\text { Ottauquechee River }\end{array}$ & $\begin{array}{r}1 \mathrm{GH} \\
01-1514 \quad 00\end{array}$ & $\begin{array}{ll}43 & 36 \\
72 & 22\end{array}$ & 220 & 1,105 & - & - & 71,400 & 25 & FR & 1961 & Corps of Engineers & PA \\
\hline $\begin{array}{l}\text { North Springfield } \\
\text { Black River }\end{array}$ & $\begin{array}{r}1 \mathrm{GH} \\
01-152900\end{array}$ & $\begin{array}{ll}43 & 20 \\
72 & 31\end{array}$ & 158 & 1,200 & - & - & 51,100 & 25 & $F R$ & 1960 & Corps of Engineers & $\mathrm{PA}$ \\
\hline $\begin{array}{l}\text { Somerset } \\
\text { East Branch Deerfield River }\end{array}$ & $\begin{array}{r}1 \mathrm{GL} \\
01-167490\end{array}$ & $\begin{array}{ll}42 & 58 \\
72 & 57\end{array}$ & 300 & 1,623 & 0 & 57,345 & 57,345 & 10 & $P$ & 1913 & $\underset{\text { Co }}{\text { New England Power }}$ & PA \\
\hline $\begin{array}{l}\text { Townshend } \\
\text { West River }\end{array}$ & $\begin{array}{r}1 \mathrm{GH} \\
01-155900\end{array}$ & $\begin{array}{ll}43 & 03 \\
72 & 42\end{array}$ & 278 & 735 & - & - & 33,500 & 08 & $\mathrm{FR}$ & 1961 & Corps of Engineers & PA \\
\hline $\begin{array}{l}\text { Union Village } \\
\text { Ompompanoosuc River }\end{array}$ & $\begin{array}{r}1 G G \\
01-141000\end{array}$ & $\begin{array}{ll}43 & 48 \\
72 & 15\end{array}$ & 126 & 720 & 20 & 38,000 & 38,000 & 28 & $F R$ & 1949 & Corps of Engineers & $\mathrm{PA}$ \\
\hline $\begin{array}{l}\text { Waterbury } \\
\text { Little River }\end{array}$ & $\begin{array}{r}4 P G \\
04-288500\end{array}$ & $\begin{array}{ll}44 & 23 \\
72 & 46\end{array}$ & 109 & 1,330 & 500 & 65,100 & 64,600 & 4 & FP & 1938 & State of vermont & PA \\
\hline $\begin{array}{l}\text { Wrightsville Detention } \\
\text { North Branch Winooski River } \\
\text { VIRGINIA }\end{array}$ & $04-2850 \quad 00$ & $\begin{array}{ll}44 & 19 \\
72 & 34\end{array}$ & 665 & 580 & 197 & 20,200 & 20,000 & 2 & F & 1935 & State of vermont & PA \\
\hline $\begin{array}{l}\text { Carvins Cove } \\
\text { Carvins Creek }\end{array}$ & $2 \mathrm{CCA}$ & $\begin{array}{ll}37 & 23 \\
79 & 57\end{array}$ & 18 & 630 & 1,500 & 19,800 & 18,300 & 14 & MR & 1945 & C1ty of Roanoke & - \\
\hline $\begin{array}{l}\text { Claytor Lake } \\
\text { New River }\end{array}$ & $03-1690 \quad 00$ & $\begin{array}{ll}37 & 04 \\
80 & 35\end{array}$ & 2,382 & 4,540 & 132,000 & 232,000 & 100,000 & 05 & PR & 1939 & $\begin{array}{l}\text { Appalachian Power } \\
\text { Co }\end{array}$ & $\mathrm{PA}$ \\
\hline $\begin{array}{l}\text { Cohoon Pond } \\
\text { Nansemond River }\end{array}$ & O2-0428 $2 \mathrm{BA}$ & $\begin{array}{ll}36 & 45 \\
76 & 38\end{array}$ & 30 & 585 & 150 & 5,210 & 5,060 & 2 & M & 1918 & $\begin{array}{c}\text { Portsmouth Water } \\
\text { Department }\end{array}$ & - \\
\hline $\begin{array}{l}\text { John H Kerr } \\
\text { Roanoke River }\end{array}$ & 2CF & $\begin{array}{ll}36 & 36 \\
78 & 18\end{array}$ & a7,780 & 95,500 & 697,900 & $2,808,400$ & $2,110,500$ & 4 & FNPRY & 1952 & Corps of Engineers & PA \\
\hline $\begin{array}{l}\text { Lake Burnt M1lls } \\
\text { Western Branch Everetts Creek }\end{array}$ & $02-0428 \quad 50$ & $\begin{array}{ll}36 & 50 \\
76 & 38\end{array}$ & 25 & 700 & 0 & 10,400 & 10,400 & 5 & M & 1943 & C1ty of Norfolk & - \\
\hline $\begin{array}{l}\text { Lake Drummond } \\
\text { Lake Drummond Outlet }\end{array}$ & $02-043000$ & $\begin{array}{ll}36 & 36 \\
76 & 27\end{array}$ & a.140 & 3,200 & 0 & 22,000 & 22,000 & - & $\mathrm{N}$ & 1936 & Corps of Engineers & $\mathrm{PA}$ ef \\
\hline $\begin{array}{l}\text { Lake Prince } \\
\text { Exchange Creek }\end{array}$ & 2BA & $\begin{array}{ll}36 & 48 \\
76 & 37\end{array}$ & 301 & 946 & 0 & 11,300 & 11,300 & 5 & M & 1921 & Clty of Norfolk & - \\
\hline
\end{tabular}




\begin{tabular}{|c|c|c|c|c|c|c|c|c|c|c|c|}
\hline $\begin{array}{l}\text { Leesville Lake } \\
\text { Roanoke (Staunton) River }\end{array}$ & $\left|\begin{array}{r}2 \mathrm{CB} \\
02-0594 \quad 00\end{array}\right|$ & $\begin{array}{ll}37 & 06 \\
79 & 24\end{array}$ & 1,505 & a3, 400 & 34,560 & 112,900 & b37,900 & $<05$ & MPR & 1962 & $\underset{\text { Co }}{\text { Appalachian Power }}$ \\
\hline $\begin{array}{l}\text { L1ttle Creek (includes Lakes Sm1th, } \\
\text { Lawson, and Wr1ght) } \\
\text { Little Creek }\end{array}$ & $02-0429^{2 \mathrm{BB}} 00$ & $\begin{array}{ll}36 & 55 \\
76 & 11\end{array}$ & 136 & 1,064 & 0 & 6,380 & 6,380 & 6 & M & 1898 & C1ty of Norfolk \\
\hline $\begin{array}{l}\text { Occoquan } \\
\text { Occoquan Creek }\end{array}$ & | $191-657700$ & $\begin{array}{ll}38 & 42 \\
77 & 17\end{array}$ & 594 & - & 0 & 30,689 & 30,689 & 09 & MP & 1958 & $\begin{array}{l}\text { Alexandria water } \\
\text { Co }\end{array}$ \\
\hline $\begin{array}{l}\text { Philpott } \\
\text { SmIth River }\end{array}$ & $02-0719^{2 \mathrm{CC}} 00$ & $\begin{array}{ll}36 & 47 \\
80 & 02\end{array}$ & 212 & 4,970 & 56,300 & 201,500 & 145,200 & 7 & FPRW & 1951 & Corps of Engineers \\
\hline $\begin{array}{l}\text { Talbott } \\
\text { Dan River }\end{array}$ & $02-0678^{2 C C} 00$ & $\begin{array}{ll}36 & 4.1 \\
80 & 24\end{array}$ & 202 & 165 & 0 & $\mathrm{~d} 8,030$ & $\mathrm{~d} 8,030$ & 2 & $\mathrm{P}$ & 1939 & C1ty of Danville \\
\hline \multicolumn{12}{|l|}{ WASHINGTON } \\
\hline $\begin{array}{l}\text { Alder } \\
\text { N1squally River }\end{array}$ & $\begin{array}{r}12 \mathrm{GK} \\
12-085000\end{array}$ & $\begin{array}{rr}46 & 48 \\
122 & 18\end{array}$ & 287 & 2,300 & 52,300 & 231,900 & 179,600 & 2 & MP & 1944 & C1ty of Tacoma \\
\hline $\begin{array}{l}\text { Baker Lake } \\
\text { Baker River }\end{array}$ & $12-1916$ ODD & \begin{tabular}{rr|}
48 & 39 \\
121 & 41
\end{tabular} & 215 & 64,985 & 64,840 & $\mathrm{~b} 285,470$ & 220,630 & 2 & $P$ & 1959 & $\begin{array}{l}\text { Puget Sound Power } \\
\text { and Light co }\end{array}$ \\
\hline $\begin{array}{l}\text { Banks Lake (Grand Coulee Equalizing } \\
\text { Reservolr) } \\
\text { Columbia River c/ }\end{array}$ & $12-435600$ & $\begin{array}{rr}47 & 37 \\
119 & 16\end{array}$ & - & 27,000 & 513,000 & $1,275,100$ & 762,100 & - & IR & 1949 & $\begin{array}{l}\text { Bureau of } \\
\text { Reclamation }\end{array}$ \\
\hline $\begin{array}{l}\text { Bonneville } \\
\text { Columb1a River }\end{array}$ & $\begin{array}{r}14 \mathrm{BG} \\
14-128850\end{array}$ & $\begin{array}{rr}45 & 49 \\
121 & 57\end{array}$ & 240,000 & 20,400 & 450,000 & b719,000 & b87,000 & $<05$ & NP & 1938 & Corps of EngIneers \\
\hline $\begin{array}{l}\text { Bumping Lake } \\
\text { Bumping River }\end{array}$ & $12-487500$ & $\begin{array}{rr}46 & 52 \\
121 & 18\end{array}$ & 68 & 1,310 & 0 & 33,700 & 33,700 & 2 & I & 2910 & $\begin{array}{l}\text { Bureau of } \\
\text { Reclamation }\end{array}$ \\
\hline $\begin{array}{l}\text { Chester Morse Lake (Cedar Lake Dam) } \\
\text { Cedar R1ver }\end{array}$ & $12-1159$ OG & $\begin{array}{rr}47 & 25 \\
121 & 44\end{array}$ & 81 & 1,830 & 38,137 & 75,323 & 37,186 & 3 & MP & 1915 & City of Seattle \\
\hline $\begin{array}{l}\text { Clear Creek } \\
\text { North Fork Tieton River }\end{array}$ & $\begin{array}{r}12 \mathrm{FO} \\
12-4904 \text { OO }\end{array}$ & $\begin{array}{rr}46 & 38 \\
121 & 16\end{array}$ & 60 & 265 & 0 & 5,300 & 5,300 & 05 & I & 1918 & $\begin{array}{l}\text { Bureau of } \\
\text { Reclamation }\end{array}$ \\
\hline $\begin{array}{l}\text { Cle Elum } \\
\text { Cle Elum RIver }\end{array}$ & $12-478500$ & $\begin{array}{rr}47 & 15 \\
121 & 04\end{array}$ & 202 & 4,810 & 0 & 436,900 & 436,900 & 7 & I & 1908 & $\begin{array}{l}\text { Bureau of } \\
\text { Reclamation }\end{array}$ \\
\hline $\begin{array}{l}\text { Conconully } \\
\text { Salmon Creek }\end{array}$ & $12-446480$ & $\begin{array}{rr}48 & 32 \\
119 & 45\end{array}$ & 121 & 420 & 0 & 13,000 & 13,000 & 5 & I & 1911 & $\begin{array}{l}\text { Okanogan Irrigation } \\
\text { District }\end{array}$ \\
\hline $\begin{array}{l}\text { Diablo } \\
\text { Skagit R1ver }\end{array}$ & $12-176500$ & $\begin{array}{rr}48 & 43 \\
121 & 08\end{array}$ & 1,125 & 928 & b81, 000 & 89,220 & $b 8,220$ & $<05$ & MP & 1930 & C1ty of Seattle \\
\hline $\begin{array}{l}\text { Franklin D Roosevelt Lake (Grand } \\
\text { Coulee Dam) } \\
\text { Columbla River }\end{array}$ & $\begin{array}{r}12 \mathrm{FC} \\
12-436000\end{array}$ & $\begin{array}{rr}47 & 57 \\
118 & 59\end{array}$ & 74,100 & 79,400 & $4,329,800$ & $d 9,562,000$ & $\mathrm{~d} 5,232,000$ & 06 & IP & 1941 & $\begin{array}{l}\text { Bureau of } \\
\text { Reclamation }\end{array}$ \\
\hline $\begin{array}{l}\text { Gorge } \\
\text { Skag1t River }\end{array}$ & $\left|\begin{array}{r}12 G C \\
12-177700\end{array}\right|$ & $\begin{array}{rr}48 & 42 \\
121 & 12\end{array}$ & 1,159 & 241 & 0 & 8,485 & $\mathrm{~b} 2,285$ & $<05$ & $\mathrm{P}$ & 1960 & City of Seattle \\
\hline
\end{tabular}



[For meaning of symbols under "Use" and "Storage records published," see text]

\begin{tabular}{|c|c|c|c|c|c|c|c|c|c|c|c|c|}
\hline \multirow[b]{2}{*}{$\begin{array}{c}\text { Name of reservo1r } \\
\text { and } \\
\text { stream }\end{array}$} & \multicolumn{2}{|c|}{ Location } & \multirow{2}{*}{$\begin{array}{c}\text { Drainage } \\
\text { area } \\
\text { (square } \\
\text { m1les) }\end{array}$} & \multirow[b]{2}{*}{$\begin{array}{c}\text { Surface } \\
\text { area } \\
\text { (acres) }\end{array}$} & \multicolumn{3}{|c|}{ Storage in acre-feet } & \multirow[b]{2}{*}{$\begin{array}{c}\text { Storage } \\
\text { rat10 }\end{array}$} & \multirow[b]{2}{*}{ Use } & \multirow[b]{2}{*}{$\begin{array}{c}\text { Date } \\
\text { com- } \\
\text { pleted }\end{array}$} & \multirow[b]{2}{*}{$\begin{array}{l}\text { Owner } \\
\text { or } \\
\text { operator }\end{array}$} & \multirow{2}{*}{$\begin{array}{l}\text { Storage } \\
\text { records } \\
\text { pub- } \\
\text { l1shed }\end{array}$} \\
\hline & \begin{tabular}{|c|} 
Basin \\
1ndex and \\
station no
\end{tabular} & $\begin{array}{l}\text { Lat1tude } \\
\text { and } \\
\text { long1tude }\end{array}$ & & & Dead & Total & Usable & & & & & \\
\hline \multicolumn{13}{|l|}{ WASHINGTON--Cont1nued } \\
\hline $\begin{array}{l}\text { Keechelus Lake } \\
\text { Yakima River }\end{array}$ & $\begin{array}{r}12 \mathrm{FN} \\
12-474000\end{array}$ & $\begin{array}{rr}47 & 19 \\
121 & 20\end{array}$ & 55 & 2,560 & 0 & 152,800 & 152,800 & 06 & I & 1917 & $\begin{array}{l}\text { Bureau of } \\
\text { Reclamation }\end{array}$ & PA \\
\hline $\begin{array}{l}\text { Lake Aldwell } \\
\text { Elwha River }\end{array}$ & $\begin{array}{r}12 \mathrm{GN} \\
12-045900\end{array}$ & $\begin{array}{rr}48 & 06 \\
123 & 33\end{array}$ & 308 & 580 & - & 33,000 & - & - & $\mathrm{P}$ & 1911 & $\begin{array}{l}\text { Crown Zellerbach } \\
\text { Corp }\end{array}$ & - \\
\hline $\begin{array}{l}\text { Lake Celilo (The Dalles Dam)* } \\
\text { Columbia River }\end{array}$ & $\begin{array}{r}14 \mathrm{BG} \\
14-103900\end{array}$ & $\begin{array}{rr}45 & 37 \\
121 & 07\end{array}$ & 237,000 & b11,650 & 280,000 & 332,500 & $b 52,500$ & $<05$ & NPR & 1957 & Corps of Engineers & - \\
\hline $\begin{array}{l}\text { Lake Chaplain } \\
\text { Sultan River } \mathrm{c}\end{array}$ & $12-138100$ & $\begin{array}{rr}47 & 57 \\
121 & 50\end{array}$ & - & 350 & 644 & 14,060 & 13,420 & - & м & 1943 & C1ty of Everett & - \\
\hline $\begin{array}{l}\text { Lake Chelan } \\
\text { Chelan River }\end{array}$ & $\begin{array}{r}12 \mathrm{FH} \\
12-4520 \text { OO }\end{array}$ & $\begin{array}{rr}47 & 50 \\
120 & 01\end{array}$ & 951 & 31,975 & 0 & 676,100 & 676,100 & 5 & $\mathrm{PR}$ & 1927 & $\mid$\begin{tabular}{|} 
helan County Publid \\
Ut1lities Dist Nb.1
\end{tabular} & PA \\
\hline $\begin{array}{l}\text { Lake Cushman No } 1 \\
\text { North Fork Skokom1sh River }\end{array}$ & $\begin{array}{r}12 \mathrm{GM} \\
12-057000\end{array}$ & $\begin{array}{rr}47 & 25 \\
123 & 13\end{array}$ & 94 & 4,000 & 81,500 & 443,994 & 362,494 & 7 & $\mathrm{P}$ & 1926 & City of Tacoma & - \\
\hline $\begin{array}{l}\text { Lake Entiat (Rocky Reach Dam) } \\
\text { Columbla River }\end{array}$ & $\mid \begin{array}{r}12 \mathrm{FJ} \\
12-453680\end{array}$ & $\begin{array}{rr}47 & 32 \\
122 & 18\end{array}$ & $a 88,500$ & 10,200 & 37,100 & 101,400 & 64,300 & $<05$ & $P$ & 1961 & $\begin{array}{r}\text { Chelan County Public } \\
\text { Ut111t1es D1st.No } 1\end{array}$ & - \\
\hline $\begin{array}{l}\text { Lake Kachess } \\
\text { Kachess R1ver }\end{array}$ & $\begin{array}{r}12 \mathrm{FN} \\
12-475500\end{array}$ & $\begin{array}{rr}47 & 16 \\
121 & 12\end{array}$ & 64 & 4,535 & 0 & 239,000 & 239,000 & 11 & I & 1912 & $\begin{array}{l}\text { Bureau of } \\
\text { Reclamation }\end{array}$ & PA \\
\hline $\begin{array}{l}\text { Lake Merwin (Ariel) } \\
\text { Lewis River }\end{array}$ & $\begin{array}{r}14 \mathrm{AJ} \\
14-220000\end{array}$ & $\begin{array}{rr}45 & 57 \\
122 & 33\end{array}$ & 730 & 3,922 & b159,000 & $\mathrm{d} 422,400$ & d263,400 & 08 & $P$ & 1931 & $\begin{array}{l}\text { Pac1fic Power and } \\
\text { L1ght Co }\end{array}$ & PA \\
\hline $\begin{array}{l}\text { Lake Mills (Glines Canyon Dam) } \\
\text { Elwha River }\end{array}$ & $\begin{array}{r}12 \mathrm{GN} \\
12-045000\end{array}$ & $\begin{array}{rr}48 & 03 \\
123 & 35\end{array}$ & 245 & 435 & 26,000 & 37,790 & 11,790 & $<05$ & $\mathrm{P}$ & 1927 & $\begin{array}{l}\text { Crown Zelierbach } \\
\text { Corp }\end{array}$ & PA \\
\hline $\begin{array}{l}\text { Lake Shannon } \\
\text { Baker R1ver }\end{array}$ & $12-193000$ & $\begin{array}{rr}48 & 33 \\
121 & 44\end{array}$ & 297 & $b 2,218$ & 17,100 & 159,470 & d142,370 & 08 & $\mathrm{P}$ & 1925 & $\begin{array}{l}\text { Puget Sound Power } \\
\text { and Light co }\end{array}$ & PA \\
\hline $\begin{array}{l}\text { Lake Tapps } \\
\text { White RIver } c /\end{array}$ & $12-101000$ & $\begin{array}{rr}47 & 14 \\
122 & 10\end{array}$ & - & b2, 566 & 이 & 46,660 & 46,660 & - & $\mathrm{P}$ & 1911 & $\begin{array}{l}\text { Puget Sound Power } \\
\text { and Light Co }\end{array}$ & PA \\
\hline $\begin{array}{l}\text { Lake Wallula (McNary Dam) * } \\
\text { Columbla River }\end{array}$ & $\begin{array}{r}14 \mathrm{BA} \\
14-019190\end{array}$ & $\begin{array}{rr}45 & 56 \\
119 & 18\end{array}$ & 214,000 & 42,500 & $1,250,000$ & $1,350,000$ & b100,000 & $<05$ & CINPR & 1957 & Corps of Eng1neers & - \\
\hline $\begin{array}{l}\text { Lake whatcom } \\
\text { Whatcom Creek }\end{array}$ & $12-202500$ & $\begin{array}{rr}48 & 46 \\
122 & 25\end{array}$ & 56 & 4,800 & 0 & b26, 400 & b26,400 & 7 & M & 1937 & C1ty of Bellingham & - \\
\hline $\begin{array}{l}\text { Long Lake } \\
\text { Columbia River of }\end{array}$ & $12-4357$ OFM & $\begin{array}{rr}47 & 27 \\
119 & 15\end{array}$ & - & 1,020 & 43,000 & 65,000 & 22,000 & - & I & 1948 & $\begin{array}{l}\text { Bureau of } \\
\text { Reclamation }\end{array}$ & - \\
\hline $\begin{array}{l}\text { Long Lake } \\
\text { Spokane River }\end{array}$ & $\left|\begin{array}{r}12 \mathrm{EK} \\
12-432500\end{array}\right|$ & $\begin{array}{rr}47 & 50 \\
117 & 50\end{array}$ & 5,920 & 5,020 & 148,600 & 252,800 & 104,200 & $<05$ & $\mathrm{P}$ & 1913 & $\begin{array}{l}\text { Washington Water } \\
\text { Power Co }\end{array}$ & PA \\
\hline
\end{tabular}

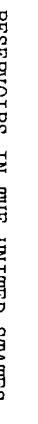




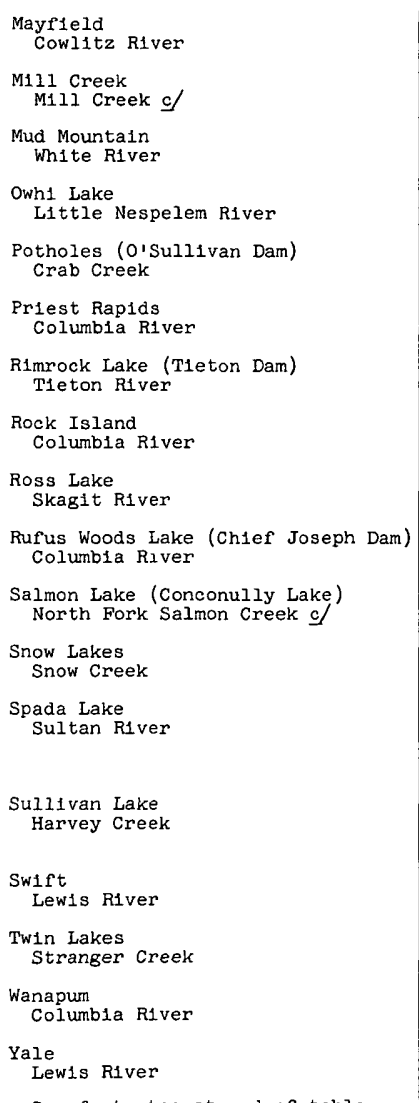

\begin{tabular}{|c|c|c|c|c|}
\hline $\begin{array}{r}14 \mathrm{AK} \\
14-237800\end{array}$ & $\begin{array}{rr}46 & 30 \\
122 & 35\end{array}$ & al, 400 & 2,25 이 & 105,620 \\
\hline $\begin{array}{r}14 \mathrm{BB} \\
14-012800\end{array}$ & \begin{tabular}{rr|}
46 & 04 \\
118 & 16
\end{tabular} & - & 225 & 79 \\
\hline $\begin{array}{r}12 G J \\
12-098000\end{array}$ & $\begin{array}{rr}47 & 08 \\
121 & 56\end{array}$ & 400 & 960 & 0 \\
\hline $\begin{array}{r}12 \mathrm{FC} \\
12-4376 \text { OO }\end{array}$ & \begin{tabular}{rr|}
48 & 13 \\
118 & 53
\end{tabular} & 132 & 540 & 0 \\
\hline $\begin{array}{r}12 \mathrm{FM} \\
12-471480\end{array}$ & $\begin{array}{rr}46 & 59 \\
119 & 16\end{array}$ & $a 4,150$ & 28,500 & 106,000 \\
\hline $\begin{array}{r}12 \mathrm{FM} \\
12-472700\end{array}$ & $\begin{array}{rr}46 & 38 \\
119 & 55\end{array}$ & a95,500 & 7,700 & 154,300 \\
\hline $\begin{array}{r}12 F 0 \\
12-491000\end{array}$ & $\begin{array}{rr}46 & 39 \\
121 & 07\end{array}$ & 187 & 2,530 & 0 \\
\hline $\begin{array}{r}12 \mathrm{FM} \\
12-462550\end{array}$ & $\begin{array}{rr}47 & 21 \\
120 & 06\end{array}$ & $a 89,600$ & 3,680 & - \\
\hline $\begin{array}{r}12 \mathrm{GC} \\
12-175000\end{array}$ & $\begin{array}{rr}48 & 44 \\
121 & 04\end{array}$ & 999 & 10,700 & 1,175 \\
\hline $\begin{array}{r}12 \mathrm{FC} \\
12-4379 \text { O0 }\end{array}$ & $\begin{array}{rr}48 & 00 \\
119 & 38\end{array}$ & a75,000 & 7,800 & 400,800 \\
\hline $\begin{array}{r}12 \mathrm{FD} \\
12-446450\end{array}$ & $\begin{array}{rr}48 & 34 \\
119 & 44\end{array}$ & - & 320 & a 12,000 \\
\hline $\begin{array}{r}\text { I2FL } \\
12-458100\end{array}$ & $\begin{array}{rr}47 & 29 \\
120 & 44\end{array}$ & 49 & 184 & $a 4,000$ \\
\hline $\begin{array}{r}12 \mathrm{GF} \\
12-137200\end{array}$ & $\begin{array}{rr}47 & 58 \\
121 & 41\end{array}$ & 68 & 600 & 0 \\
\hline $\begin{array}{r}12 \mathrm{DN} \\
12-397000\end{array}$ & $\begin{array}{rr}48 & 50 \\
117 & 17\end{array}$ & 518 & 1,293 & - \\
\hline $\begin{array}{r}14 \text { AJ } \\
14-217600\end{array}$ & $\begin{array}{rr}46 & 04 \\
122 & 12\end{array}$ & 481 & 4,621 & 308,585 \\
\hline $\begin{array}{r}\text { 12ED } \\
12-4099 \text { OD }\end{array}$ & $\begin{array}{rr}48 & 16 \\
118 & 22\end{array}$ & $\begin{array}{ll}37 & 1\end{array}$ & 1,892 & 0 \\
\hline $\begin{array}{r}12 F M \\
12-464610\end{array}$ & $\begin{array}{rr}4653 \\
119 & 58\end{array}$ & a 95,000 & - & 530,000 \\
\hline $\begin{array}{r}14 \mathrm{AJ} \\
14-218500\end{array}$ & $\begin{array}{r}4558 \\
122 \quad 20\end{array}$ & 596 & 3,783 & 212,231 \\
\hline
\end{tabular}

$\begin{array}{r}127,000 \\ 8,000 \\ 106,000 \\ 5,250 \\ 525,100 \\ 198,700 \\ 198,000 \\ 8,600 \\ 1,405,300 \\ 518,000 \\ a 22,500 \\ 16,500 \\ 20,000 \\ 15,124 \\ 669,700 \\ 755,600 \\ \hline\end{array}$
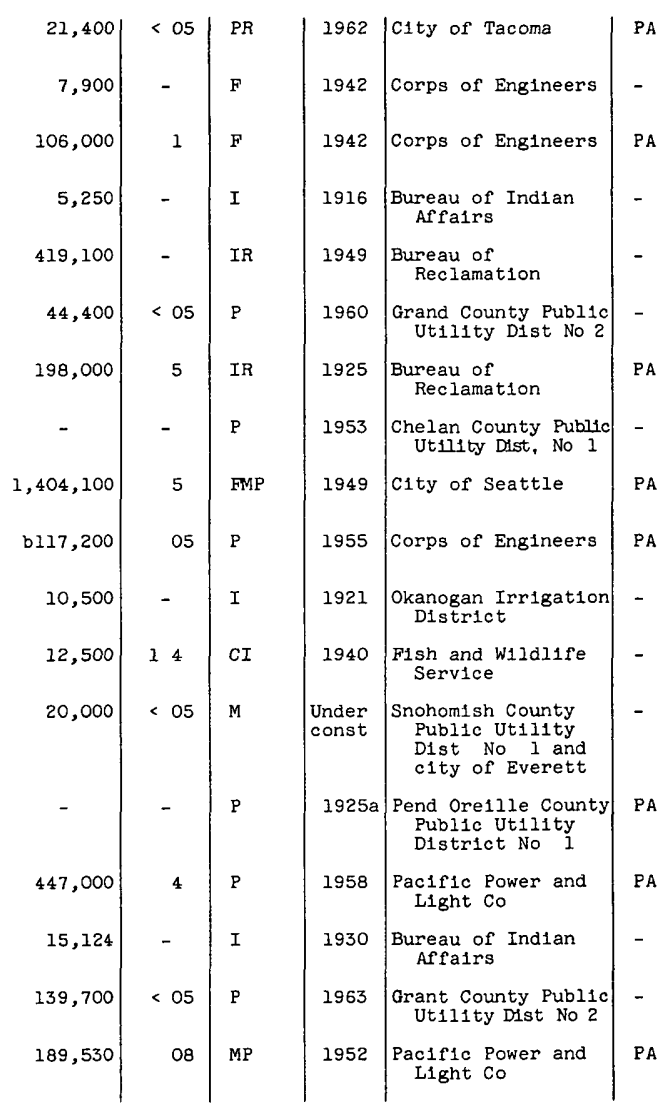
Table 1 --Reservo1rs in the United States completed or under construction as of January 1, 1963, having a usable capac1ty of 5,000 acre-feet or more--Continued [For meaning of symbols under "Use" and "Storage records published," see text]

\begin{tabular}{|c|c|c|c|c|c|c|c|c|c|c|c|c|}
\hline \multirow[b]{2}{*}{$\begin{array}{l}\text { Name of reservolr } \\
\text { and } \\
\text { stream }\end{array}$} & \multicolumn{2}{|c|}{ Location } & \multirow{2}{*}{$\begin{array}{l}\text { Drainage } \\
\text { area } \\
\text { (square } \\
\text { m1les) }\end{array}$} & \multirow[b]{2}{*}{$\begin{array}{c}\text { Surface } \\
\text { area } \\
\text { (acres) }\end{array}$} & \multicolumn{3}{|c|}{ Storage in acre-feet } & \multirow[b]{2}{*}{$\mid \begin{array}{c}\text { Storage } \\
\text { rat1o }\end{array}$} & \multirow[b]{2}{*}{ Use } & \multirow[b]{2}{*}{$\begin{array}{c}\text { Date } \\
\text { com- } \\
\text { pleted }\end{array}$} & \multirow[b]{2}{*}{$\begin{array}{l}\text { Owner } \\
\text { or } \\
\text { operator }\end{array}$} & \multirow{2}{*}{$\begin{array}{l}\text { Storage } \\
\text { records } \\
\text { pub- } \\
\text { lished }\end{array}$} \\
\hline & $\begin{array}{c}\text { Basin } \\
\text { Index and } \\
\text { station no }\end{array}$ & $\begin{array}{l}\text { Lat1 tude } \\
\text { and } \\
\text { long1 tude }\end{array}$ & & & Dead & Total & Usable & & & & & \\
\hline WEST VIRGINIA & & & & & & & & & & & & \\
\hline $\begin{array}{l}\text { Bluestone } \\
\text { New River }\end{array}$ & $\begin{array}{r}3 F D \\
03-179800\end{array}$ & $\begin{array}{ll}37 & 38 \\
80 & 53\end{array}$ & 4,602 & 9,178 & 10,000 & 631,000 & 621,000 & 02 & $\mathrm{~F}$ & 1952 & Corps of Eng1neers & - \\
\hline $\begin{array}{l}\text { Sutton } \\
\text { Elk River }\end{array}$ & $\begin{array}{r}3 \mathrm{FL} \\
03-195400\end{array}$ & $\begin{array}{ll}38 & 40 \\
80 & 42\end{array}$ & 537 & 3,875 & 4,100 & 265,300 & 261,200 & 3 & FWV & 1960 & Corps of Engineers & PA \\
\hline $\begin{array}{l}\text { Tygart Lake } \\
\text { Tygart River }\end{array}$ & $\begin{array}{r}3 B B \\
03-055500\end{array}$ & $\begin{array}{ll}39 & 19 \\
80 & 02\end{array}$ & 1,184 & 3,430 & 2,950 & b287, 700 & b287, 700 & 2 & FNR & 1938 & Corps of Engineers & PA \\
\hline WISCONSIN & & & & & & & & & & & & \\
\hline $\begin{array}{l}\text { Alexander } \\
\text { Wisconsin River }\end{array}$ & $05-394170$ & $\begin{array}{ll}45 & 11 \\
89 & 45\end{array}$ & 2,520 & 803 & - & a7,, 000 & - & - & $\mathrm{P}$ & 1924 & $\begin{array}{l}\text { Wisconsin Public } \\
\text { Service Corp }\end{array}$ & - \\
\hline $\begin{array}{l}\text { Altoona Lake } \\
\text { Eau Cla1re River }\end{array}$ & $\begin{array}{r}5 \mathrm{CK} \\
05-366530\end{array}$ & $\begin{array}{ll}44 & 49 \\
91 & 26\end{array}$ & 830 & 947 & 0 & a10,000 & $a 10,000$ & $<05$ & $\mathrm{R}$ & 1939 & Eau Cla1re County & - \\
\hline $\begin{array}{l}\text { Bear Lake } \\
\text { Bear Creek }\end{array}$ & O5-3674 50 & $\begin{array}{ll}45 & 37 \\
91 & 47\end{array}$ & 60 & 2,055 & - & 10,900 & 9,500 & - & $P$ & 1880 & $\begin{array}{l}\text { Northern States } \\
\text { Power Co }\end{array}$ & $\mathrm{PA}$ \\
\hline $\begin{array}{l}\text { Beaverdam Lake } \\
\text { Beaverdam R1ver }\end{array}$ & $05-4259^{5 F D} 10$ & $\begin{array}{ll}43 & 27 \\
88 & 50\end{array}$ & 139 & 5,440 & - & - & 5,000 & 1 & $\mathrm{R}$ & 1840 & City of Beaverdam & - \\
\hline $\begin{array}{l}\text { Big Eau Pleine } \\
\text { Big Eau Pleine River }\end{array}$ & $\begin{array}{r}5 E C \\
05-399600\end{array}$ & $\begin{array}{ll}44 & 44 \\
89 & 46\end{array}$ & 365 & 6,998 & 0 & 102,300 & 102,300 & - & $P$ & 1937 & $\begin{array}{l}\text { Wisconsin Valley } \\
\text { Improvement Co }\end{array}$ & PA \\
\hline $\begin{array}{l}\text { B1rch Lake } \\
\text { Red Cedar River }\end{array}$ & O5-3671 5 OO & $\begin{array}{ll}45 & 40 \\
91 & 33\end{array}$ & 68 & 3,400 & 6,500 & 27,345 & $b 18,110$ & - & PR & 1882 & $\begin{array}{l}\text { Northern States } \\
\text { Power Co }\end{array}$ & $\mathrm{PA}$ \\
\hline $\begin{array}{l}\text { Biron } \\
\text { W1sconsin River } c \text { / }\end{array}$ & $05-400730$ & $\begin{array}{ll}44 & 26 \\
89 & 47\end{array}$ & - & 2,087 & - & a20,000 & - & - & PW & 1887 & $\begin{array}{l}\text { Consolidated Water } \\
\text { Power Co }\end{array}$ & - \\
\hline $\begin{array}{l}\text { Brule * } \\
\text { Brule River }\end{array}$ & $\begin{array}{r}4 \mathrm{CJ} \\
04-0620 \quad 10\end{array}$ & $\begin{array}{ll}45 & 57 \\
88 & 13\end{array}$ & 1,050 & 774 & - & 18,000 & - & - & $\mathrm{P}$ & 1919 & $\begin{array}{l}\text { W1sconsin-Michigan } \\
\text { Power Co }\end{array}$ & - \\
\hline $\begin{array}{l}\text { Buffalo Lake (Montello Dam) } \\
\text { Fox R1ver }\end{array}$ & $\begin{array}{r}4 \mathrm{DC} \\
04-072930\end{array}$ & $\begin{array}{ll}43 & 48 \\
89 & 20\end{array}$ & 601 & 2,370 & - & - & 25,000 & $<05$ & $\mathrm{c}$ & - & Corps of Engineers & - \\
\hline $\begin{array}{l}\text { Burnt Roliways } \\
\text { Eagle R1ver }\end{array}$ & $05-390350$ & $\begin{array}{ll}45 & 54 \\
89 & 08\end{array}$ & 129 & 7,657 & - & 14,540 & 12,070 & - & $\mathrm{PR}$ & 1908 & $\begin{array}{l}\text { Wisconsin Valley } \\
\text { Improvement Co }\end{array}$ & PA \\
\hline $\begin{array}{l}\text { Caldron Falls } \\
\text { Peshtigo RIver }\end{array}$ & 04-067980 & $\begin{array}{ll}45 & 21 \\
88 & 14\end{array}$ & 496 & 1,212 & - & - & 25,000 & $<05$ & $P$ & 1924 & $\begin{array}{l}\text { W1sconsin Public } \\
\text { Service Corp }\end{array}$ & - \\
\hline $\begin{array}{l}\text { Castle Rock } \\
\text { Wisconsin River }\end{array}$ & |05-4032 500 & $\begin{array}{ll}43 & 52 \\
89 & 58\end{array}$ & 6,864 & 16,640 & - & 175,000 & 98,000 & - & PR & 1950 & $\begin{array}{l}\text { Wisconsin River } \\
\text { Power Co }\end{array}$ & $\mathrm{PA}$ \\
\hline
\end{tabular}




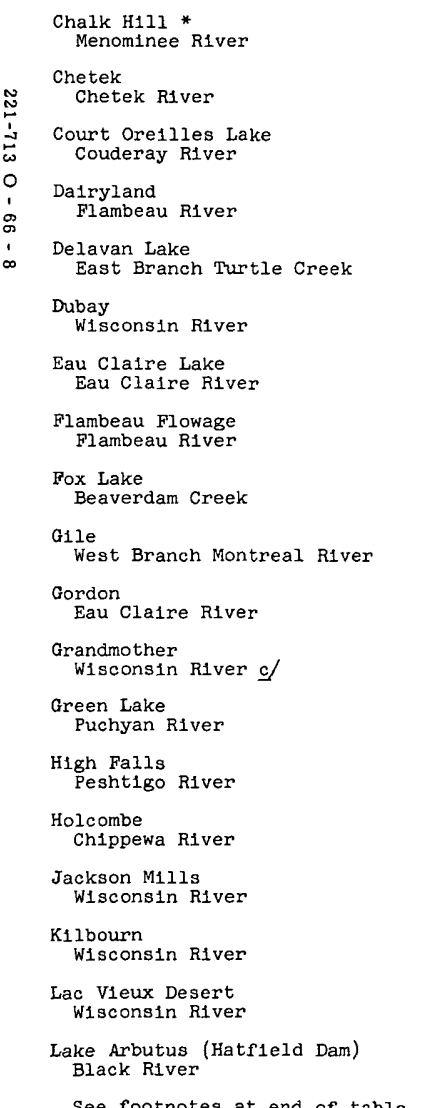

\begin{tabular}{|c|c|c|c|c|}
\hline $\begin{array}{r}4 \mathrm{CL} \\
04-066020\end{array}$ & $\begin{array}{ll}45 & 31 \\
87 & 48\end{array}$ & 3,321 & 765 & - \\
\hline $\begin{array}{r}\text { 5CL } \\
05-3674 \quad 70\end{array}$ & $\begin{array}{ll}45 & 19 \\
91 & 39\end{array}$ & 177 & 3,750 & - \\
\hline $\begin{array}{r}5 \mathrm{CG} \\
05-356100\end{array}$ & $\begin{array}{ll}45 & 51 \\
91 & 24\end{array}$ & 86 & 5,250 & - \\
\hline $\begin{array}{r}5 \mathrm{CH} \\
05-3600 \quad 20\end{array}$ & $\begin{array}{ll}45 & 29 \\
91 & 03\end{array}$ & 1,928 & 1,950 & - \\
\hline $05-4310^{5 \mathrm{FE}} 20$ & $\begin{array}{ll}42 & 37 \\
88 & 39\end{array}$ & 36 & 1,813 & - \\
\hline $05-4002^{5 E C} 95$ & $\begin{array}{ll}44 & 39 \\
89 & 39\end{array}$ & 4,890 & 7,052 & - \\
\hline $\begin{array}{r}5 \mathrm{CK} \\
05-366200\end{array}$ & $\begin{array}{ll}44 & 46 \\
91 & 08\end{array}$ & 605 & 1,800 & - \\
\hline $\begin{array}{r}5 \mathrm{CH} \\
05-3574 \quad 0 \mathrm{O}\end{array}$ & $\begin{array}{ll}46 & 04 \\
90 & 14\end{array}$ & 666 & 17,800 & 0 \\
\hline $05-425890$ & $\begin{array}{ll}43 & 34 \\
88 & 55\end{array}$ & 52 & 2,456 & - \\
\hline $\begin{array}{r}4 \mathrm{AK} \\
04-028995\end{array}$ & $\begin{array}{ll}46 & 26 \\
90 & 14\end{array}$ & 78 & 3,400 & 0 \\
\hline $\begin{array}{r}5 \mathrm{CB} \\
05-331600\end{array}$ & $\begin{array}{ll}46 & 14 \\
91 & 47\end{array}$ & 99 & 2,500 & - \\
\hline $05-3936 \frac{5 \mathrm{~EB}}{60}$ & $\begin{array}{ll}45 & 22 \\
89 & 44\end{array}$ & - & 758 & - \\
\hline $\begin{array}{r}4 \mathrm{DC} \\
04-0734 \quad 70\end{array}$ & $\begin{array}{ll}43 & 51 \\
88 & 58\end{array}$ & 115 & 7,325 & - \\
\hline $04-068000$ & $\begin{array}{ll}45 & 17 \\
88 & 12\end{array}$ & 554 & 1,750 & - \\
\hline $\begin{array}{r}5 \mathrm{CJ} \\
05-3625 \quad 10\end{array}$ & $\begin{array}{ll}45 & 14 \\
91 & 08\end{array}$ & 4,700 & 4,250 & - \\
\hline $05-4003 \frac{5 E C}{20}$ & $\begin{array}{ll}44 & 31 \\
89 & 35\end{array}$ & 4,964 & 1,310 & - \\
\hline $05-4036 \frac{5 \mathrm{FE}}{15}$ & $\begin{array}{ll}43 & 38 \\
89 & 47\end{array}$ & 7,746 & 2,150 & - \\
\hline $\begin{array}{r}\text { 5EA } \\
05-390100\end{array}$ & $\begin{array}{ll}46 & 07 \\
89 & 09\end{array}$ & 28 & 5,066 & 0 \\
\hline $\begin{array}{r}5 C R \\
05-381180\end{array}$ & $\begin{array}{ll}44 & 25 \\
90 & 44\end{array}$ & 1,290 & 1,200 & $\mathrm{~b} 2,060$ \\
\hline
\end{tabular}

\begin{tabular}{|c|c|c|}
\hline$a 8,000$ & - & - \\
\hline $\mathrm{d} 23,000$ & $\mathrm{~d} 6,000$ & 07 \\
\hline - & $a 5,000$ & - \\
\hline$a 50,000$ & a14,000 & $<05$ \\
\hline - & 5,000 & - \\
\hline 2102,000 & 49,000 & - \\
\hline - & 10,000 & $\triangleleft 05$ \\
\hline 135,500 & 135,500 & 3 \\
\hline - & a5, 000 & 3 \\
\hline 29,600 & 29,600 & 5 \\
\hline - & d7, 000 & 1 \\
\hline a5, 000 & - & - \\
\hline - & b5, 000 & 08 \\
\hline - & $b 5,000$ & $<05$ \\
\hline 46,000 & 31,000 & - \\
\hline$a 8,000$ & - & - \\
\hline$a 20,000$ & - & - \\
\hline 15,000 & 15,000 & - \\
\hline $\mathrm{d} 8,310$ & $\mathrm{~d} 6,250$ & - \\
\hline
\end{tabular}

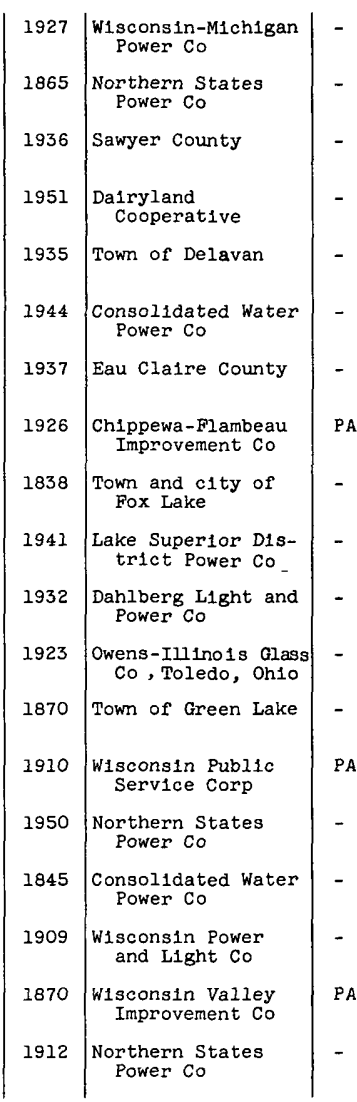




\begin{tabular}{|c|c|c|c|c|c|c|c|c|c|c|c|c|}
\hline \multirow[b]{2}{*}{$\begin{array}{c}\text { Name of reservolr } \\
\text { and } \\
\text { stream }\end{array}$} & \multicolumn{2}{|c|}{ Location } & \multirow{2}{*}{$\begin{array}{l}\text { Drainage } \\
\text { area } \\
\text { (square } \\
\text { miles) }\end{array}$} & \multirow[b]{2}{*}{$\begin{array}{c}\text { Surface } \\
\text { area } \\
\text { (acres) }\end{array}$} & \multicolumn{3}{|c|}{ Storage in acre-feet } & \multirow[b]{2}{*}{$\mid \begin{array}{c}\text { Storage } \\
\text { rat10 }\end{array}$} & \multirow[b]{2}{*}{ Use } & \multirow[b]{2}{*}{$\begin{array}{c}\text { Date } \\
\text { com- } \\
\text { pleted }\end{array}$} & \multirow[b]{2}{*}{$\begin{array}{l}\text { Owner } \\
\text { or } \\
\text { operator }\end{array}$} & \multirow{2}{*}{$\begin{array}{l}\text { Storage } \\
\text { recorås } \\
\text { pub- } \\
\text { l1shed }\end{array}$} \\
\hline & \begin{tabular}{|c|} 
Basin \\
1ndex and \\
station no
\end{tabular} & $\begin{array}{l}\text { Lat1 tude } \\
\text { and } \\
\text { long1tude }\end{array}$ & & & Dead & Total & Usable & & & & & \\
\hline WISCONSIN--Cont1nued & & & & & & & & & & & & \\
\hline $\begin{array}{l}\text { Lake Chippewa } \\
\text { Chippewa River }\end{array}$ & $\begin{array}{r}5 \mathrm{CG} \\
05-355600\end{array}$ & $\begin{array}{ll}45 & 53 \\
91 & 05\end{array}$ & 775 & 15,300 & 0 & 229,600 & 229,600 & 05 & $\mathrm{PR}$ & 1923 & $\begin{array}{l}\text { Northern States } \\
\text { Power } \mathrm{Co}^{\circ}\end{array}$ & PA \\
\hline $\begin{array}{l}\text { Lake Geneva } \\
\text { White River }\end{array}$ & $\begin{array}{r}5 K F \\
05-545180\end{array}$ & $\begin{array}{ll}42 & 35 \\
88 & 25\end{array}$ & 36 & 5,500 & - & - & a15, 000 & 9 & $R$ & 1912 & $\begin{array}{l}\text { Lake Geneva Water } \\
\text { Power and Lake } \\
\text { Level Assoc }\end{array}$ & - \\
\hline $\begin{array}{l}\text { Lake Koshkonong (Indian Ford Dam) } \\
\text { Rock River }\end{array}$ & $05-427560$ & $\begin{array}{ll}42 & 48 \\
89 & 05\end{array}$ & 2,594 & 9,890 & - & - & b5, 000 & - & $\mathrm{P}$ & 1905 & $\begin{array}{l}\text { Wisconsin Power } \\
\text { and Light Co }\end{array}$ & - \\
\hline $\begin{array}{l}\text { Lake Mendota } \\
\text { Yahara R1ver }\end{array}$ & $05-4280 \quad 5 \mathrm{FE}$ & $\begin{array}{ll}43 & 06 \\
89 & 22\end{array}$ & 254 & 9,730 & - & - & $b 20,000$ & 3 & $\mathrm{R}$ & 1849 & C1ty of Madison & $\mathrm{PA} e /$ \\
\hline $\begin{array}{l}\text { Lake Monona and Lake Waubesa } \\
\text { Yahara River }\end{array}$ & $05-429495$ & $\begin{array}{ll}43 & 01 \\
89 & 18\end{array}$ & 350 & 5,517 & - & - & b5, 000 & - & $\mathrm{R}$ & 1938 & Dane County & PA \\
\hline $\begin{array}{l}\text { Lake Nokom1s (Rice) } \\
\text { Tomahawk R1ver }\end{array}$ & 5EA & $\begin{array}{ll}45 & 32 \\
89 & 45\end{array}$ & 545 & 4,435 & 0 & 41,500 & 41,500 & 1 & $P$ & 1912 & $\begin{array}{l}\text { W1 sconsin Valley } \\
\text { Improvement Co }\end{array}$ & PA \\
\hline $\begin{array}{l}\text { Lake Noquebay (Noque Lake) } \\
\text { Lake Noquebay Outlet }\end{array}$ & $04-069300$ & $\begin{array}{ll}45 & 15 \\
87 & 56\end{array}$ & 118 & 2,419 & - & - & 25,000 & - & $\mathrm{CR}$ & 1885 & $\begin{array}{l}\text { Lake Noque Bay } \\
\text { Improvement Assoc }\end{array}$ & - \\
\hline $\begin{array}{l}\text { Lake Puckaway } \\
\text { Fox RIver }\end{array}$ & 04-0731 $\begin{array}{r}4 D C \\
60\end{array}$ & $\begin{array}{ll}43 & 50 \\
89 & 09\end{array}$ & 868 & 5,433 & - & - & a5, 000 & - & $\mathrm{R}$ & 1898 & $\begin{array}{l}\text { Wisconsin Conserva- } \\
\text { tion Department }\end{array}$ & - \\
\hline $\begin{array}{l}\text { Lake Winnebago } \\
\text { Fox RIver }\end{array}$ & 04-0825 $\begin{array}{r}4 \mathrm{DG} \\
00\end{array}$ & $\begin{array}{ll}44 & 01 \\
88 & 32\end{array}$ & 6,030 & 202,240 & 0 & 582,000 & 582,000 & 2 & NP & 1848 & Corps of Eng1neers & PA \\
\hline $\begin{array}{l}\text { Lake Wisconsin (Prairie du Sac Dam) } \\
\text { Wisconsin River }\end{array}$ & $05-405990$ & $\begin{array}{ll}43 & 19 \\
89 & 43\end{array}$ & 8,944 & 9,500 & - & 180,000 & b5, 000 & - & PR & 1914 & $\begin{array}{l}\text { W1sconsin Power } \\
\text { and IIght Co }\end{array}$ & $\mathrm{PA}$ \\
\hline $\begin{array}{l}\text { Lake W1ssota } \\
\text { Chippewa River }\end{array}$ & $\begin{array}{r}5 \mathrm{CJ} \\
05-364200\end{array}$ & $\begin{array}{ll}44 & 56 \\
91 & 20\end{array}$ & 5,548 & 6,180 & - & 92,000 & 81,500 & 05 & PR & 1917 & $\begin{array}{l}\text { Northern States } \\
\text { Power Co }\end{array}$ & $\mathrm{PA}$ \\
\hline $\begin{array}{l}\text { Long Lake } \\
\text { Brill River }\end{array}$ & $05-3673$ OCL & $\begin{array}{ll}45 & 40 \\
91 & 41\end{array}$ & 82 & 3,950 & - & 14,620 & 11,700 & - & PR & 1883 & $\begin{array}{l}\text { Northern States } \\
\text { Power Co }\end{array}$ & PA \\
\hline $\begin{array}{l}\text { Long Lake } \\
\text { Deerskin River }\end{array}$ & 5EA & $\begin{array}{ll}46 & 03 \\
89 & 03\end{array}$ & 35 & 2,715 & - & 9,180 & 8,400 & - & PR & 1908 & $\begin{array}{l}\text { W1sconsin Valley } \\
\text { Improvement Co }\end{array}$ & PA \\
\hline $\begin{array}{l}\text { Metonga Lake } \\
\text { Swamp Creek tributary }\end{array}$ & $\begin{array}{r}4 \mathrm{DD} \\
04-074510\end{array}$ & $\begin{array}{ll}45 & 31 \\
88 & 54\end{array}$ & 16 & 3,513 & - & - & 5,000 & 4 & $\mathrm{CR}$ & 1926 & $\begin{array}{l}\text { Forest County } \\
\text { Protective Assoc }\end{array}$ & - \\
\hline $\begin{array}{l}\text { Minocqua Lake } \\
\text { Tomahawk River }\end{array}$ & $\begin{array}{r}\text { 5EA } \\
05-392100\end{array}$ & $\begin{array}{ll}45 & 53 \\
89 & 44\end{array}$ & 89 & 6,729 & 0 & 14,420 & 14,420 & - & PR & 1890 & $\begin{array}{l}\text { W1sconsin Valley } \\
\text { Improvement Co }\end{array}$ & PA \\
\hline
\end{tabular}



Minong (Lake Nancy Flowage)

Moose Lake

West Fork Ch1ppewa River

Mosinee

W1sconsin River

Nelson Lake (Totagatic)
Totagatic River

Okauchee Lake

Oconowoc River

Otter Rapids
W1sconsin RIver

Pelican Lake

Pelican River

Petenwell

Wisconsin River

Pewaukee Lake

Phillips Flowage (Jobe Dam)

Elk River

Pucherel Laino
St Germain River

Rainbow

Wiscons1n River

Red Cedar Lake

Rest Lake

Monitowish River

St Crolx Falls *

St Crolx Flowage

Croix River

Sp1rit

Spirit River

Spooner Lake

Sugar Camp
Sugar Ca

reek

See footnotes at end of table

\begin{tabular}{|c|c|c|c|c|c|}
\hline $\begin{array}{r}5 C B \\
05-3331 \quad 60\end{array}$ & $\begin{array}{ll}46 & 07 \\
91 & 56\end{array} \mid$ & 320 & 1,700 & - & $a 6,000$ \\
\hline $\begin{array}{r}5 \mathrm{CG} \\
05-3554 \quad 00\end{array}$ & $\begin{array}{ll}46 & 02 \\
91 & 04\end{array}$ & 225 & 1,800 & 0 & 9,200 \\
\hline $\begin{array}{r}5 \mathrm{EC} \\
05-3986 \quad 00\end{array}$ & $\begin{array}{ll}44 & 47 \\
89 & 42\end{array}$ & 4,126 & 1,380 & - & $a 6,880$ \\
\hline $\begin{array}{r}5 \mathrm{CB} \\
05-3330 \quad 50\end{array}$ & $\begin{array}{ll}46 & 06 \\
91 & 31\end{array}$ & 90 & 2,800 & - & 20,000 \\
\hline $05-425040$ & $\begin{array}{ll}43 & 07 \\
88 & 26\end{array}$ & 84 & 1,057 & - & - \\
\hline $\begin{array}{r}5 E A \\
05-3906 \quad 30\end{array}$ & $\begin{array}{ll}45 & 54 \\
89 & 19\end{array}$ & 533 & 3,250 & - & a15,000 \\
\hline $\begin{array}{r}5 E A \\
05-391100\end{array}$ & $\begin{array}{ll}45 & 32 \\
89 & 12\end{array}$ & 22 & 3,450 & 0 & 7,000 \\
\hline $05-4014$ SED & $\begin{array}{ll}44 & 03 \\
90 & 01\end{array}$ & 5,860 & 23,040 & - & 456,000 \\
\hline $\begin{array}{r}5 \mathrm{KF} \\
05-5438 \quad 15\end{array}$ & $\begin{array}{ll}43 & 05 \\
88 & 16\end{array}$ & 20 & 2,300 & - & - \\
\hline $05-3594 \quad \begin{array}{r}5 \mathrm{CH} \\
\mathrm{OO}\end{array}$ & $\begin{array}{ll}45 & 41 \\
90 & 27\end{array}$ & 195 & 2,000 & - & - \\
\hline $05-3908^{\frac{55 \Delta}{00}}$ & $\begin{array}{ll}45 & 57 \\
89 & 32\end{array}$ & 78 & 1130 & - & 7,760 \\
\hline $05-3909$ 5EA & $\begin{array}{ll}45 & 50 \\
89 & 33\end{array}$ & 740 & 4,485 & 0 & 50,100 \\
\hline $\begin{array}{r}5 \mathrm{CL} \\
05-367200\end{array}$ & $\begin{array}{ll}45 & 35 \\
91 & 36\end{array}$ & 161 & 2,800 & o & 13,200 \\
\hline $05-3573$ OCH & $\begin{array}{ll}46 & 08 \\
84 & 53\end{array}$ & 243 & 4,200 & - & 23,200 \\
\hline $\begin{array}{r}5 C D \\
05-3404 \quad 95\end{array}$ & $\begin{array}{ll}45 & 25 \\
92 & 39\end{array}$ & 5,930 & 840 & 0 & $\mathrm{~d} 5,080$ \\
\hline $\begin{array}{r}5 \mathrm{CB} \\
05-3316 \quad 20\end{array}$ & $\begin{array}{ll}46 & 15 \\
91 & 56\end{array}$ & 282 & 3,300 & - & - \\
\hline $05-393600$ & $\begin{array}{ll}45 & 26 \\
89 & 44\end{array}$ & 174 & 2,096 & 0 & 17,400 \\
\hline $\begin{array}{r}5 \mathrm{CB} \\
05-333980\end{array}$ & $\begin{array}{ll}45 & 50 \\
91 & 50\end{array}$ & 20 & 1,212 & - & - \\
\hline $\begin{array}{r}5 E A \\
05-390650\end{array}$ & $\begin{array}{ll}45 & 52 \\
89 & 24\end{array}$ & 59 & 2,209 & - & 10,800 \\
\hline
\end{tabular}

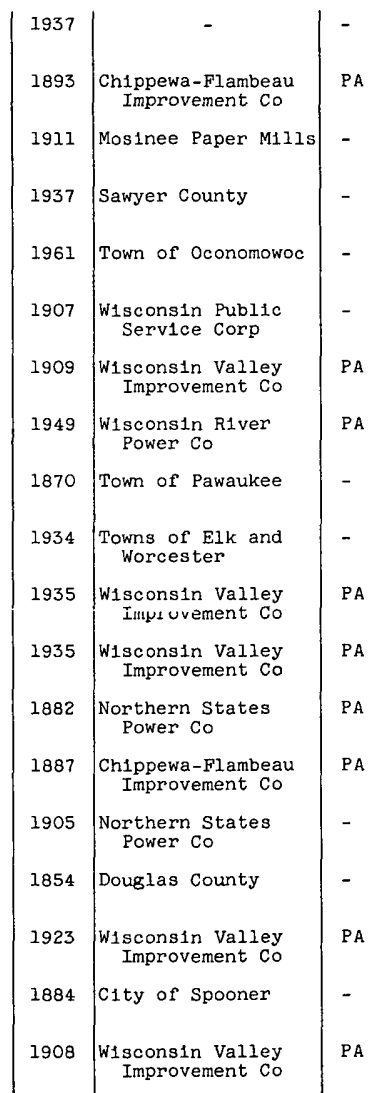

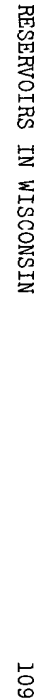




\begin{tabular}{|c|c|c|c|c|c|c|c|c|c|c|c|c|}
\hline \multirow[b]{2}{*}{$\begin{array}{l}\text { Name of reservolr } \\
\text { and } \\
\text { stream }\end{array}$} & \multicolumn{2}{|c|}{ Location } & \multirow{2}{*}{$\begin{array}{l}\text { Drainage } \\
\text { area } \\
\text { (square } \\
\text { mlles) }\end{array}$} & \multirow[b]{2}{*}{$\begin{array}{c}\text { Surface } \\
\text { area } \\
\text { (acres) }\end{array}$} & \multicolumn{3}{|c|}{ Storage in acre-feet } & \multirow[b]{2}{*}{$\begin{array}{c}\text { Storage } \\
\text { rat10 }\end{array}$} & \multirow[b]{2}{*}{ Use } & \multirow[b]{2}{*}{$\begin{array}{c}\text { Date } \\
\text { com- } \\
\text { pleted }\end{array}$} & \multirow[b]{2}{*}{$\begin{array}{l}\text { Owner } \\
\text { or } \\
\text { operator }\end{array}$} & \multirow{2}{*}{$\begin{array}{l}\text { Storage } \\
\text { records } \\
\text { pub- } \\
\text { lished }\end{array}$} \\
\hline & \begin{tabular}{|c|} 
Basin \\
1ndex and \\
station no
\end{tabular} & \begin{tabular}{|l|} 
Lat1 tude \\
and \\
long1tude
\end{tabular} & & & Dead & Total & Usable & & & & & \\
\hline WISCONS IN -- Cont Inued & & & & & & & & & & & & \\
\hline $\begin{array}{l}\text { Tainter Lake } \\
\text { Red Cedar R1ver }\end{array}$ & $\begin{array}{r}5 \mathrm{CM} \\
05-368495\end{array}$ & $\begin{array}{ll}44 & 56 \\
91 & 53\end{array}$ & 1,680 & 1,800 & - & - & $d 5,000$ & - & $P$ & 1853 & $\begin{array}{l}\text { Northern States } \\
\text { Power Co }\end{array}$ & - \\
\hline $\begin{array}{l}\text { Tigercat Lake } \\
\text { Chief River }\end{array}$ & $\begin{array}{r}5 C G \\
05-3554 \quad 50\end{array}$ & $\begin{array}{ll}46 & 02 \\
91 & 15\end{array}$ & 47 & 2,230 & - & - & a7, 000 & 02 & $\mathrm{CR}$ & 1938 & Sawyer County & - \\
\hline $\begin{array}{l}\text { Twin Lakes } \\
\text { Tw1n R1ver }\end{array}$ & $\begin{array}{r}5 \mathrm{EA} \\
05-390150\end{array}$ & $\begin{array}{ll}46 & 01 \\
89 & 10\end{array}$ & 26 & 3,616 & - & 7,190 & 6,400 & - & $\mathrm{P}$ & 1908 & $\begin{array}{l}\text { Wisconsin Valley } \\
\text { Improvement Co }\end{array}$ & PA \\
\hline $\begin{array}{l}\text { White Rapids * } \\
\text { Menominee River }\end{array}$ & $\begin{array}{r}4 \mathrm{CL} \\
04-066030\end{array}$ & $\begin{array}{ll}45 & 29 \\
87 & 48\end{array}$ & 3,325 & 445 & - & 26,000 & - & - & $P$ & 1927 & $\begin{array}{l}\text { W1 sconsin-M1chigan } \\
\text { Power Co }\end{array}$ & - \\
\hline $\begin{array}{l}\text { W1llow } \\
\text { Tomahawk R1ver }\end{array}$ & $05-392300$ & $\begin{array}{ll}45 & 43 \\
89 & 51\end{array}$ & 327 & 7,368 & - & - & 75,800 & - & $P$ & 1927 & $\begin{array}{l}\text { Wisconsin Valley } \\
\text { Improvement Co }\end{array}$ & PA \\
\hline $\begin{array}{l}\text { Wiscons1n Rapids } \\
\text { W1sconsin River }\end{array}$ & $05-4007 \begin{array}{r}5 \mathrm{ED} \\
60\end{array}$ & $\begin{array}{ll}44 & 23 \\
89 & 50\end{array}$ & 5,391 & 470 & - & 6,000 & - & - & $P$ & 1843 & $\begin{array}{l}\text { Consolidated water } \\
\text { Power Co }\end{array}$ & - \\
\hline WYOMING & & & & & & & & & & & & \\
\hline $\begin{array}{l}\text { Alcova } \\
\text { North Platte RIver }\end{array}$ & $06-6415^{6 N F} 00$ & $\begin{array}{rr}42 & 33 \\
106 & 43\end{array}$ & 10,075 & 2,250 & 100 & 190,500 & 190,400 & 2 & IP & 1938 & $\begin{array}{l}\text { Bureau of } \\
\text { Reclamation }\end{array}$ & PA \\
\hline $\begin{array}{l}\text { Anchor } \\
\text { South Fork Owl Creek }\end{array}$ & $06-2603^{6 \mathrm{HF}}$ & $\begin{array}{rr}43 & 40 \\
108 & 49\end{array}$ & - & - & 0 & 17,420 & 17,420 & - & I & 1960 & $\begin{array}{l}\text { Bureau of } \\
\text { Reclamation }\end{array}$ & $\mathrm{PA}$ \\
\hline $\begin{array}{l}\text { Big Goose Park } \\
\text { East Fork Big Goose Creek }\end{array}$ & $\begin{array}{r}6 \mathrm{GE} \\
06-300600\end{array}$ & \begin{tabular}{rr|}
44 & 34 \\
107 & 13
\end{tabular} & - & 304 & - & 8,908 & - & - & I & 1933 & Park Reservolr co & - \\
\hline $\begin{array}{l}\text { Big Sandy } \\
\text { Big Sandy Creek }\end{array}$ & $09-21379 \begin{array}{r}9 A E \\
00\end{array}$ & $\begin{array}{rr}42 & 15 \\
109 & 23\end{array}$ & 439 & 2,600 & 1,400 & 39,700 & 35,000 & - & I & 1952 & $\begin{array}{l}\text { Bureau of } \\
\text { Reclamation }\end{array}$ & - \\
\hline $\begin{array}{l}\text { Boulder Lake } \\
\text { Boulder Creek }\end{array}$ & $\begin{array}{r}9 \mathrm{AC} \\
09-201800\end{array}$ & $\begin{array}{rr}42 & 52 \\
109 & 42\end{array}$ & 130 & 1,680 & - & - & b12,820 & 1 & I & 1927 & $\begin{array}{l}\text { Boulder Irrigation } \\
\text { District }\end{array}$ & - \\
\hline $\begin{array}{l}\text { Boysen } \\
\text { W1nd RIver }\end{array}$ & $\begin{array}{r}6 \mathrm{HE} \\
06-258900\end{array}$ & $\begin{array}{rr}43 & 25 \\
108 & 11\end{array}$ & 7,710 & 22,200 & 62,000 & 819,800 & 757,800 & 8 & CFIP & 1951 & $\begin{array}{l}\text { Bureau of } \\
\text { Reclamation }\end{array}$ & PA \\
\hline $\begin{array}{l}\text { Buffalo Bill } \\
\text { Shoshone River }\end{array}$ & $\begin{array}{r}6 \mathrm{HM} \\
06-281500\end{array}$ & $\begin{array}{rr}44 & 30 \\
109 & 11\end{array}$ & 1,520 & 6,711 & 0 & 421,300 & 421,300 & 5 & IP & 1909 & $\begin{array}{l}\text { Bureau of } \\
\text { Reclamation }\end{array}$ & PA \\
\hline $\begin{array}{l}\text { Bull Lake } \\
\text { Bull Lake Creek }\end{array}$ & $\begin{array}{r}6 \mathrm{HC} \\
06-224500\end{array}$ & $\begin{array}{rr}43 \quad 13 \\
109 & 02\end{array}$ & - & 3,200 & 0 & 252,000 & 152,000 & 8 & I & 1938 & $\begin{array}{l}\text { Bureau of } \\
\text { Reclamation }\end{array}$ & $\mathrm{PA}$ \\
\hline $\begin{array}{l}\text { Cheyenne No } 2 \text { (Gran1te Springs Dam) } \\
\text { M1ddle Fork Crow Creek }\end{array}$ & $\begin{array}{r}60 P \\
06-754600\end{array}$ & $\begin{array}{rr}41 & 10 \\
105 & 14\end{array}$ & a25 & 190 & 0 & 7,370 & 7,370 & - & M & 1903 & C1ty of Cheyenne & - \\
\hline
\end{tabular}


Eden No 1
B1g Sandy Creek c/

Fremont Lake
P1ne Creek

Glendo North Platte River

Grassy Lake
Grassy and Cascade Creeks

Guernsey

North Platte River

Hawk Springs

Horse Creek c/

Hay Red Creek and Brush Lake

Jackson Lake

Keyhole

Belle Fourche R1ver

Lake de Smet

iney Creek of

Lake Hattie

Laramie River c/

La Prele

La Prele Creek

New Fork Lake
New Fork R1ver

Pathrinder

North Platte River

Pilot Butte
Wind River c/

Ray Lake
South Fork Little W1nd River

Seminoe

North Platte River

Sixty-Seven

North Piney Creek c/

Sunshine

Greybull River c/

See footnotes at end of table

\begin{tabular}{|c|c|c|c|}
\hline $\mid \begin{array}{r}9 \mathrm{AE} \\
09-2136 \quad 00\end{array}$ & $\begin{array}{rr}42 & 13 \\
109 & 22\end{array}$ & - & 1,180 \\
\hline $09-1969$ 9AC & 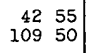 & - & 5,390 \\
\hline $\begin{array}{r}6 \mathrm{NJ} \\
06-652700\end{array}$ & $\begin{array}{rr}42 & 28 \\
104 & 58\end{array}$ & 14,330 & 18,660 \\
\hline $\begin{array}{r}13 \mathrm{EH} \\
13-0465 \mathrm{OO}\end{array}$ & $\begin{array}{rr}44 & 08 \\
110 & 49\end{array}$ & 104 & 310 \\
\hline $\begin{array}{r}6 \mathrm{NJ} \\
06-655500\end{array}$ & $\begin{array}{rr}42 & 17 \\
104 & 46\end{array}$ & 15,008 & 2,386 \\
\hline $\begin{array}{r}6 \mathrm{NN} \\
06-6766 \quad 50\end{array}$ & $\begin{array}{rr}41 & 43 \\
104 & 11\end{array}$ & - & 1,531 \\
\hline $\begin{array}{r}6 \mathrm{NM} \\
06-629880\end{array}$ & $\begin{array}{rr}42 & 01 \\
108 & 20\end{array}$ & - & 1,037 \\
\hline $\begin{array}{r}13 E A \\
13-010500\end{array}$ & $\begin{array}{rr}43 & 51 \\
110 & 35\end{array}$ & 824 & 25,540 \\
\hline $06-4270^{6 \mathrm{~KB}}$ & $\begin{array}{rr}44 & 23 \\
104 & 47\end{array}$ & 2,000 & 13,690 \\
\hline $\begin{array}{r}6 \mathrm{GE} \\
06-323100\end{array}$ & 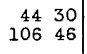 & - & 2,051 \\
\hline (06-6595 30 & $\begin{array}{rr}41 & 15 \\
105 & 55\end{array}$ & - & - \\
\hline $\begin{array}{r}6 \mathrm{NJ} \\
06-649150\end{array}$ & $\begin{array}{rr}42 & 43 \\
105 & 37\end{array}$ & al50 & 628 \\
\hline $\begin{array}{r}9 \mathrm{AC} \\
09-192800\end{array}$ & $\begin{array}{rr}43 & 05 \\
109 & 58\end{array}$ & 362 & - \\
\hline $\begin{array}{r}6 \mathrm{NF} \\
06-640500\end{array}$ & $\begin{array}{rr}42 & 28 \\
106 & 51\end{array}$ & 10,010 & 22,600 \\
\hline $\begin{array}{r}6 \mathrm{HC} \\
06-2264 \quad \mathrm{OO}\end{array}$ & $\begin{array}{rr}43 & 12 \\
108 & 45\end{array}$ & - & 900 \\
\hline $\begin{array}{r}6 \mathrm{HA} \\
06-230200\end{array}$ & $\begin{array}{rr}42 & 57 \\
108 & 50\end{array}$ & - & - \\
\hline $\begin{array}{r}6 \mathrm{NF} \\
06-6355 \text { OO }\end{array}$ & $\begin{array}{rr}42 & 09 \\
106 & 53\end{array}$ & 6,641 & 20,050 \\
\hline $\begin{array}{r}9 \mathrm{AD} \\
09-2057 \mathrm{OO}\end{array}$ & $\begin{array}{rr}42 & 36 \\
110 & 13\end{array}$ & - & 371 \\
\hline $\begin{array}{r}6 \mathrm{HJ} \\
06-274750\end{array}$ & $\begin{array}{rr}44 & 03 \\
109 & 03\end{array}$ & - & 1,159 \\
\hline
\end{tabular}

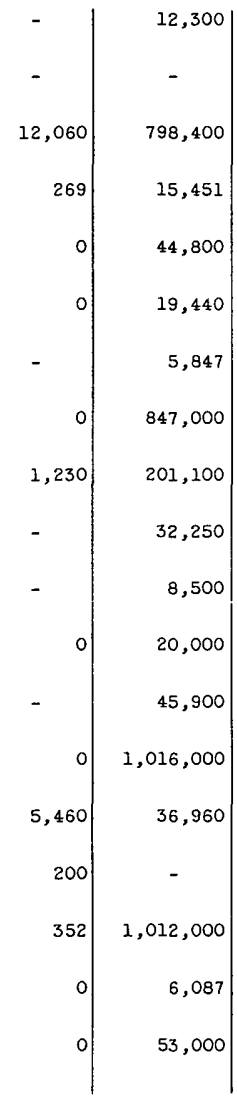

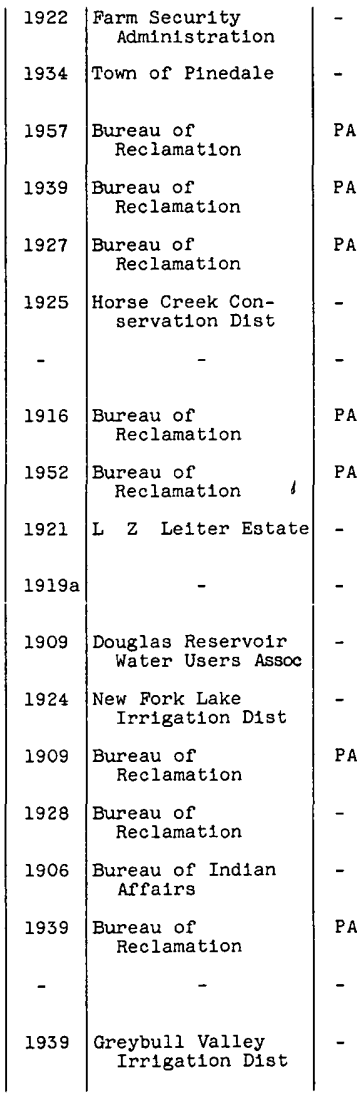



[For meaning of symbols under "Use" and "Storage records published," see text]

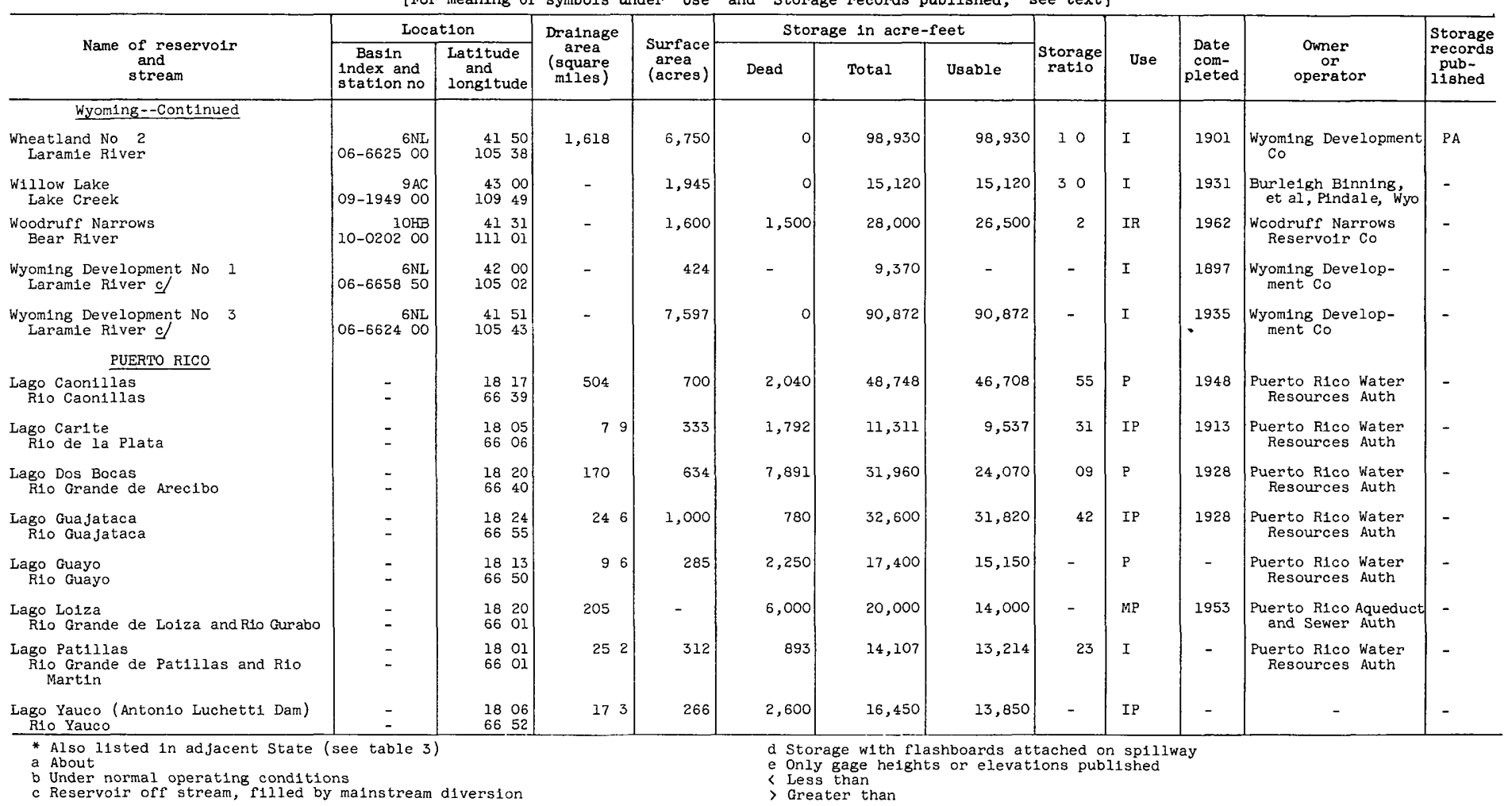




\section{SUMMARY OF RESERVOIRS BY STATES}

A summary of reservolrs showing number, usable storage, and surface area, by States, is given in table 2 A reservolr situated on a stream forming a state line is included in table 2 for only that state which is first alphabetically for example, the usable storage and surface area of Lake Mead are included only under Arizona, and this fact accounts for the large disparity in the figures shown for Arizona and Nevada

Table 2 --Summary of reservolrs showlng number, uisable storage, and surface area, by State

\begin{tabular}{|c|c|c|c|}
\hline & $\begin{array}{l}\text { Number of } \\
\text { reservoirs }\end{array}$ & $\begin{array}{c}\text { Usable storage } \\
\text { (acre-feet) }\end{array}$ & $\begin{array}{c}\text { Surface area } \\
\text { (acres) }\end{array}$ \\
\hline $\begin{array}{l}\text { Alabama } \\
\text { Alaska } \\
\text { Arizona } \\
\text { Arkansas } \\
\text { California }\end{array}$ & $\begin{array}{r}22 \\
9 \\
23 \\
15 \\
205\end{array}$ & $\begin{array}{r}4,905,100 \\
555,900 \\
56,695,200 \\
10,021,700 \\
28,368,500\end{array}$ & $\begin{array}{r}362,160 \\
9,912 \\
483,374 \\
243,093 \\
650,589\end{array}$ \\
\hline $\begin{array}{l}\text { Colorado } \\
\text { Connect1cut } \\
\text { Delaware } \\
\text { Florida } \\
\text { Georgla }\end{array}$ & $\begin{array}{r}94 \\
14 \\
1 \\
12 \\
17\end{array}$ & $\begin{array}{r}3,981,100 \\
468,800 \\
6,800 \\
3,718,200 \\
6,582,900\end{array}$ & $\begin{array}{r}129,334 \\
20,779 \\
1,200 \\
1,460,023 \\
242,918\end{array}$ \\
\hline $\begin{array}{l}\text { Hawa11 } \\
\text { Idaho } \\
\text { Illino1s } \\
\text { Indlana } \\
\text { Iowa }\end{array}$ & $\begin{array}{r}3 \\
51 \\
15 \\
9 \\
2\end{array}$ & $\begin{array}{r}23,000 \\
10,560,100 \\
203,700 \\
395,000 \\
486,400\end{array}$ & $\begin{array}{r}726 \\
532,343 \\
53,536 \\
18,488 \\
53,960\end{array}$ \\
\hline $\begin{array}{l}\text { Kansas } \\
\text { Kentucky } \\
\text { Lou1s1ana } \\
\text { Maine } \\
\text { Maryland }\end{array}$ & $\begin{array}{r}19 \\
6 \\
23 \\
76 \\
8\end{array}$ & $\begin{array}{r}4,349,100 \\
8,922,950 \\
1,497,500 \\
5,142,100 \\
472,600\end{array}$ & $\begin{array}{r}143,759 \\
345,340 \\
178,775 \\
650,038 \\
22,052\end{array}$ \\
\hline $\begin{array}{l}\text { Massachusetts } \\
\text { Michigan } \\
\text { Minnesota } \\
\text { Mississipp1 } \\
\text { Missour1 }\end{array}$ & $\begin{array}{r}24 \\
29 \\
52 \\
5 \\
6\end{array}$ & $\begin{array}{r}1,915,900 \\
551,200 \\
12,589,600 \\
3,885,900 \\
6,138,200\end{array}$ & $\begin{array}{r}55,421 \\
83,605 \\
2,271,811 \\
215,500 \\
165,100\end{array}$ \\
\hline $\begin{array}{l}\text { Montana } \\
\text { Nebraska } \\
\text { Nevada } \\
\text { New Hampsh1re } \\
\text { New Jersey }\end{array}$ & $\begin{array}{r}60 \\
19 \\
9 \\
30 \\
12\end{array}$ & $\begin{array}{r}23,286,500 \\
4,061,200 \\
655,500 \\
1,262,300 \\
219,500\end{array}$ & $\begin{array}{r}555,908 \\
113,257 \\
26,578 \\
112,600 \\
11,808\end{array}$ \\
\hline $\begin{array}{l}\text { New Mexico } \\
\text { New York } \\
\text { North Carolina } \\
\text { North Dakota } \\
\text { Oh10 }\end{array}$ & $\begin{array}{l}23 \\
72 \\
29 \\
18 \\
43\end{array}$ & $\begin{array}{r}5,412,100 \\
5,778,500 \\
4,432,300 \\
18,786,100 \\
3,390,700\end{array}$ & $\begin{array}{l}115,689 \\
371,185 \\
142,488 \\
456,671 \\
166,062\end{array}$ \\
\hline $\begin{array}{l}\text { Oklahoma } \\
\text { Oregon } \\
\text { Pennsylvania } \\
\text { Rhode Island } \\
\text { South Carolina }\end{array}$ & $\begin{array}{r}43 \\
51 \\
37 \\
2 \\
11\end{array}$ & $\begin{array}{r}10,842,000 \\
4,785,100 \\
2,066,200 \\
117,700 \\
4,148,600\end{array}$ & $\begin{array}{r}428,067 \\
212,743 \\
69,499 \\
4,574 \\
423,607\end{array}$ \\
\hline $\begin{array}{l}\text { South Dakota } \\
\text { Tennessee } \\
\text { Texas } \\
\text { Utah } \\
\text { Vermont }\end{array}$ & $\begin{array}{r}10 \\
20 \\
116 \\
37 \\
14\end{array}$ & $\begin{array}{r}30,620,600 \\
10,268,000 \\
26,460,000 \\
5,721,400 \\
532,900\end{array}$ & $\begin{array}{r}452,282 \\
373,935 \\
1,013,395 \\
197,094 \\
11,366\end{array}$ \\
\hline $\begin{array}{l}\text { V1rginia } \\
\text { Wash1ngton } \\
\text { West V1rginia } \\
\text { W1sconsin } \\
\text { Wyoming }\end{array}$ & $\begin{array}{r}12 \\
43 \\
3 \\
67 \\
33\end{array}$ & $\begin{array}{r}2,506,800 \\
12,228,000 \\
1,169,900 \\
2,008,000 \\
5,993,200\end{array}$ & $\begin{array}{r}116,100 \\
350,248 \\
16,480 \\
516,819 \\
174,933\end{array}$ \\
\hline Puerto Rico & 8 & 169,300 & 3,530 \\
\hline Total & 1,562 & $359,359,850$ & $14,830,757$ \\
\hline
\end{tabular}




\section{RESERVOIRS COMMON TO ADJACENT STATES}

A summary of those reservolrs on streams which form a boundary between adjacent States is given in table 3 The table does not include reservolrs common to adjacent States because of a river flowing across a State line

Table 3 --Reservo1rs on streams which form a boundary between adjacent states

\begin{tabular}{|c|c|c|}
\hline States & Reservolr & River \\
\hline 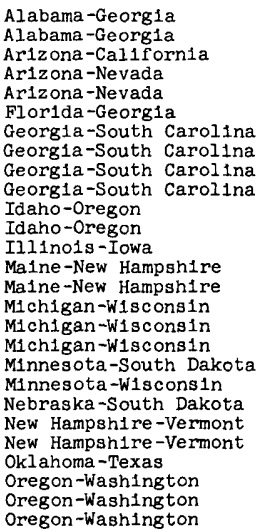 & $\begin{array}{l}\text { Goat Rock } \\
\text { Lake Harding } \\
\text { Havasu Lake } \\
\text { Lake Mead } \\
\text { Lake Mohave } \\
\text { Lake Seminole } \\
\text { Clark H11l } \\
\text { Hartwell } \\
\text { Stevens Creek } \\
\text { Tugaloo } \\
\text { Brownlee } \\
\text { Oxbow } \\
\text { Lake Cooper } \\
\text { Great East Lake } \\
\text { Three Ponds } \\
\text { Brule } \\
\text { Chalk Hill } \\
\text { Wh1te Rap1ds } \\
\text { Lake Traverse } \\
\text { St Croix Falls } \\
\text { Lew1s and Clark Lake } \\
\text { Comerford Station Pond } \\
\text { Little Development } \\
\text { Lake Texoma } \\
\text { Bonnev1lie } \\
\text { Lake Celilo } \\
\text { Lake Wallula }\end{array}$ & $\begin{array}{l}\text { Chattahoochee } \\
\text { Chattahoochee } \\
\text { Colorado } \\
\text { Colorado } \\
\text { Colorado } \\
\text { Apalach1cola } \\
\text { Savannah } \\
\text { Savannah } \\
\text { Savannah } \\
\text { Tugaloo } \\
\text { Snake } \\
\text { Snake } \\
\text { Miss1ss1pp1 } \\
\text { Salmon Falls } \\
\text { Salmon Falls } \\
\text { Brule } \\
\text { Menominee } \\
\text { Menom1nee } \\
\text { Bo1s de S1oux } \\
\text { St Croix } \\
\text { Missour1 } \\
\text { Connect1cut } \\
\text { Connect1cut } \\
\text { Red } \\
\text { Columbla } \\
\text { Columb1a } \\
\text { Columb1a }\end{array}$ \\
\hline
\end{tabular}

\section{LITERATURE CITED}

Daugherty, C R, Horton, A H , and Davenport, $\mathrm{R} W$, 1928, Power capacity and production in the United States U S Geol Survey Water-Supply Paper 579, 210 p

Draffin, J O , 1939, The story of man's quest for water, Champaign, Ill, The Garrard Press, $232 \mathrm{p}$

Federal Power Commlssion, 1964, Hydroelectric power resources of the United States, developed and undeveloped Washington, D C, $159 \mathrm{p}$

Follansbee, Robert, 1939, A history of the Water Resources Branch of the United States Geological Survey to June 30,1919 privately printed, $275 \mathrm{p}$

Harbeck, G E, Jr, 1948, Reservolrs in the United States U S Geol Survey Circ 23, $72 \mathrm{p}$

Hazen, Allen, 1914, Storage to be provided in impounding reservoirs for municipal water supply Am Soc Civil Eng Trans, v 77, p 1539-1640

Jones, B D, and Helland, R 0 , 1948 Index to river surveys made by the United States Geological Survey and other agencies, U S Geol Survey Water-Supply Paper 995, $145 \mathrm{p}$ (Originally published as a preliminary index in Water-Supply Paper 558')

Thomas, $N$, and $G$ E Harbeck, 1956, Reservoirs In the United States U S Geol Survey Watér-Supply Paper 1360-A, 99 p

Todd, J A, 1929, Cotton and the cotton 1ndustry, in Encyclopedia Brittanica [14thed ], V 6 p 536

Turneaure, F E , and Russe11, H L, 1909, Public water-supplies New York, John Wiley and Sons $\mathrm{p} 1-12$ (4th ed rev, $1940, \mathrm{p} 1-10$ ) 
INDEX

Alabama
Alaska
Arizona
Arkansas
California
Colorado
Connecticut
Delaware
Florida
Georgia
Hawai1
Idaho
Illinois
Indiana
Iowa
Kansas
Kentucky
Loulsiana
Maine
Maryland
Massachusetts
Michigan
Minnesota
Mississipp1
Missouri
Montana

Page

16 Nebraska

17 Nevada

17 New Hampshire

19 New Jersey

New Mexico

32 New York

38 North Carolina

39 North Dakota

39 On10

40 Oklahoma

41 Oregon

41 Pennsylvania

44 Puerto Rico

45 Rhode Island

46 South Carolina

46 South Dakota

47 Tennessee

47 Texas

49 Utah

53 Vermont

54 Virginia

55 Washington

57 West Virginia

60 Wisconsin

61 Wyoming

Page

65

66
67

67
69

69

71

75

77
78

83

86

112

89

89
90

90

91

99

101

102

106

106

Montana 